\title{
Who knows best? \\ Participatory Design with tenants in Wellington City Council's Housing Upgrade Programme
}

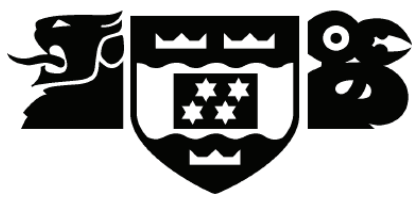

Nick Leckie

Victoria University of Wellington

School of Architecture

Submitted in partial fulfilment of the degree of Master of Architecture (Professional) 


\section{Abstract}

Public participation is the cornerstone of a democratic society and it presents very specific challenges in relation to development projects. This thesis explores participatory design - the important interface between the design of a physical project and the people who are affected by it - in reference to Wellington City Council's (WCC's) upgrading of social housing. It asks 'is there scope for widening tenant participation in the Housing Upgrade Programme?'

A literature review establishes a loose definition of participation based on seven principles: approaches to participation are diverse; often 'under-done'; cannot be neatly packaged or predicted and therefore cut across traditional professional boundaries; they engage power relations; and they tend to cultivate choice in outcomes and bring into being a collective intelligence.

From here the study engages action research techniques and case study analysis to further understanding of participatory processes. A comparative analysis of WCC's current participatory approach and that of an Architecture Sans Frontieres participatory design workshop on slum upgrading in Nairobi, Kenya is conducted which reveals room for extension in WCC's current approach and finds the Kenya workshop process exemplary. 'A design experiment' is then carried out which conducts some participatory exercises at a WCC housing site - Te Ara Hou apartments in Newtown, Wellington. These exercises contribute to a modest design proposal of additional housing units and a retrofitted community space for Te Ara Hou.

All preceding steps then inform what is the ultimate outcome of the study; a set of eight generic principles to inform best-practice participatory process. These principles are then used to evaluate three cases which demonstrates how they might be applied in practice. These three cases evaluated are WCC's existing approach, the ASF workshop approach and a proposed approach for WCC. The third case makes suggestions around how WCC could develop their participatory approach. Ultimately, the thesis finds that there is scope for widening tenant participation in the Housing Upgrade Programme, and the eight principles offer suggestions around how that might be done. 


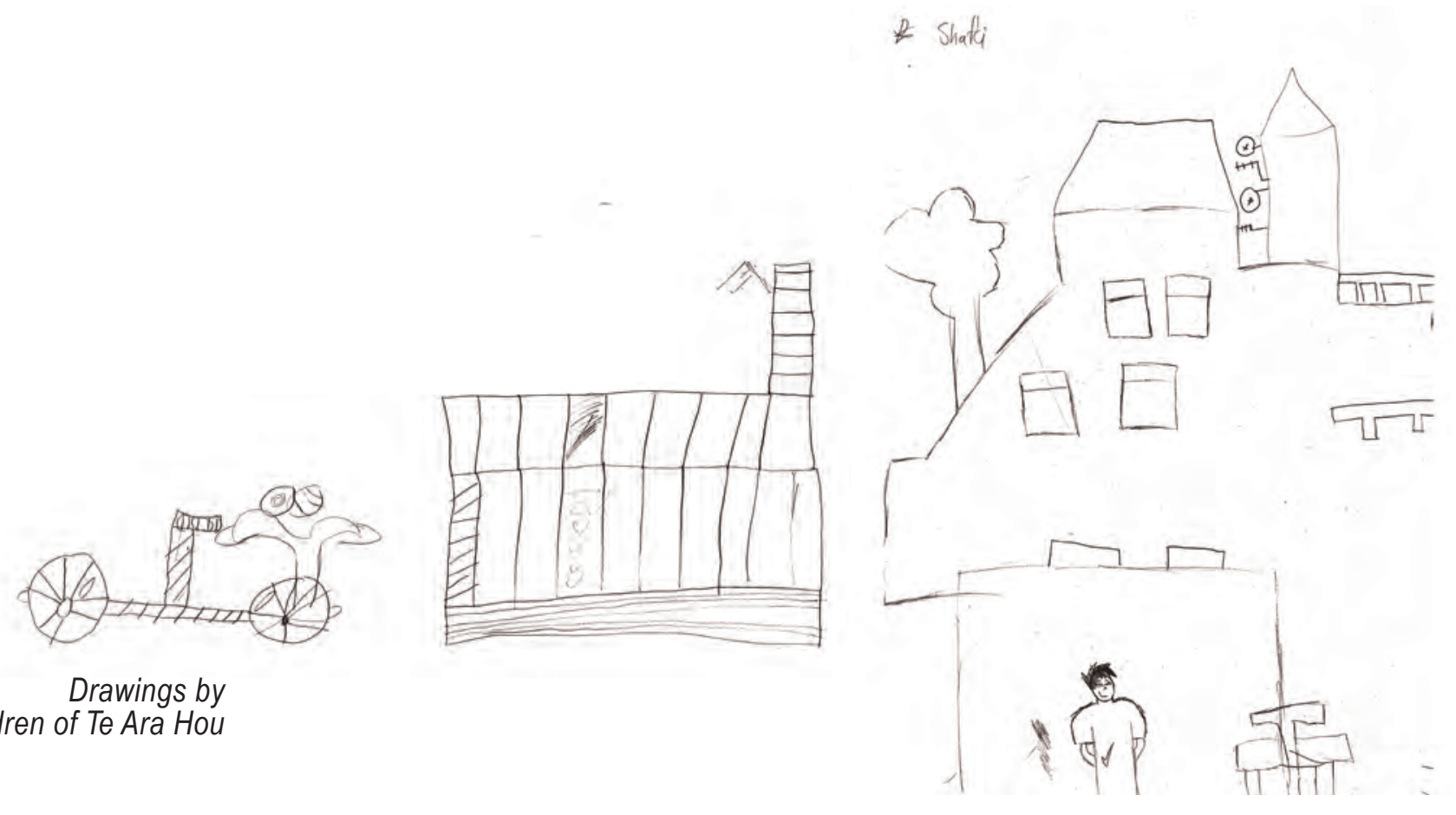



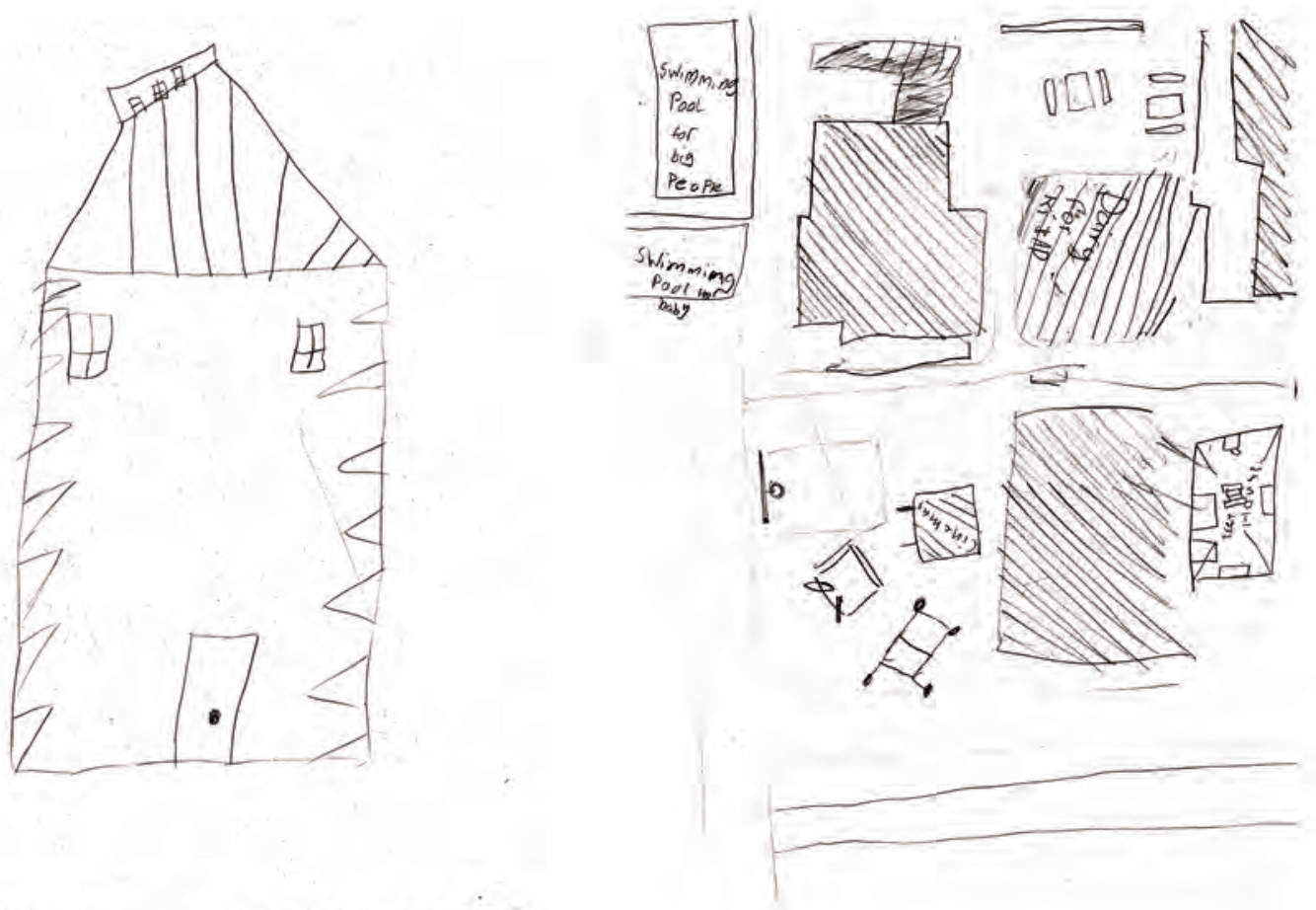


\section{Acknowledgements}

My thanks go to Chris McDonald, my supervisor, who offered me an appropriate level of freedom to explore this topic in the direction I saw fit, and offered his wisdom and clarity when I needed it. Chris' supervision of this work has been thorough and precise.

I am also grateful to the organisers of the Participatory Design workshop (by ASFUK) in Kenya, as they so capably provided me with the rich learning environment I needed to take my first steps into a participatory approach to design. Of the organisers, I especially thank Matthew French who initially encouraged me down the path of participatory design as a worthy thesis topic and has been very supportive throughout.

I would not have been able to book my tickets to the Kenya workshop without the generous financial support of the Freemasons, specifically Zetland Lodge, and STUDiO, so I express my sincere thanks to them.

Partnering this research with City Housing at Wellington City Council has made the results of the project more grounded and relevant, so I am grateful for their cooperation and patience. I especially thank Marc, Rosie, Jo and Byron.

Special thanks go to classmates James M, James $O$ and Michael who assisted me with my experimental workshops in September. Also, my profound gratitude goes to Nevada for constantly encouraging me along on this unpredictable journey and providing me with continual perspective and drive.

Finally, I thank the 16 Te Ara Hou residents who so generously gave me their time for interviews and workshops. A participatory study wouldn't have worked without participants, so I'm extremely grateful to you. You have brought all-important faces and stories to this research. 


\section{Table of Contents}

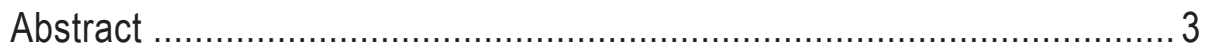

Acknowledgements ................................................................... 7

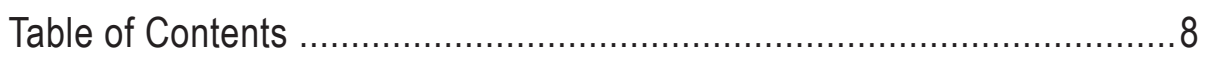

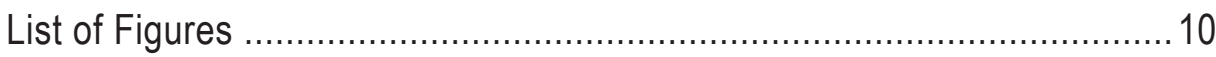

1.0 Introduction .................................................................................. 15

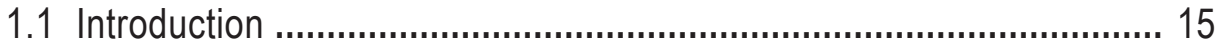

1.2 Thesis Structure ............................................................................ 17

1.3 Research Definitions, Scope and Questions ................................. 19

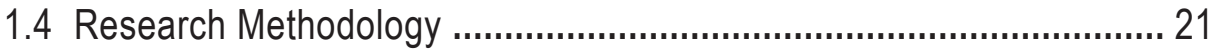

2.0 Literature Review ............................................................................... 24

2.1 Introduction ................................................................................... 24

2.2 A brief historical overview of participation ...................................... 25

2.3 Defining Participation ..................................................................... 32

3.0 WCC Housing Upgrade..................................................................... 42

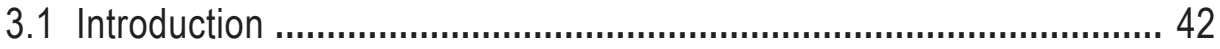

3.2 History and Future of 'Social Housing' ............................................. 44

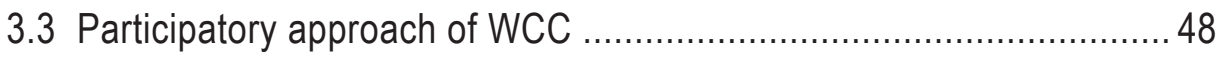

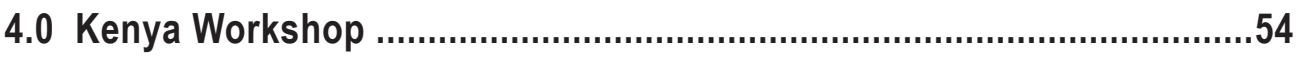

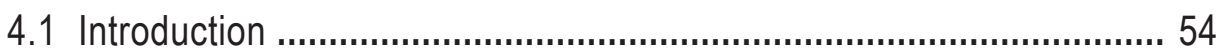

4.2 'Change by Design' - An Introduction ........................................... 55

4.3 Participatory tools and techniques .............................................. 56

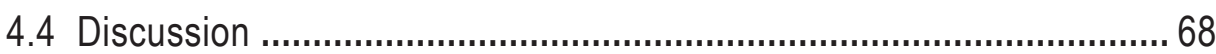

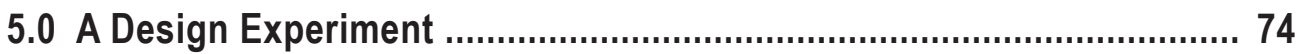

5.1 Case site .................................................................................... 75

5.2 Participatory exercises at Te Ara Hou ........................................... 83

5.3 Building understanding of elements of 'a brief' ................................. 113

5.4 Presentation of a physical intervention ......................................... 122

6.0 Developing a Participatory Approach ................................................148

6.1 Generic Principles of 'an ideal process' ........................................... 148

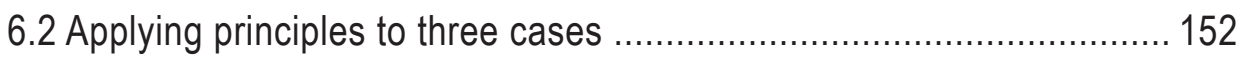

6.3 Expanding on WCC Proposed Approach .......................................... 162

7.0 Overall Conclusions and Reflections ............................................... 168

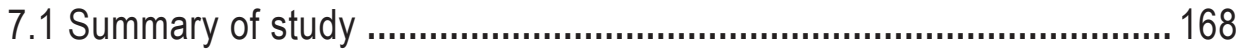

7.2 Key findings and limitations .............................................................. 169

7.3 Future directions for project ....................................................... 173

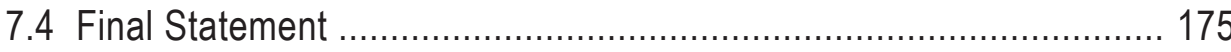




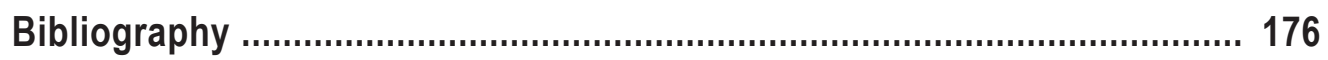

Appendices.................................................................................... 180

Appendix A - Interview guide ............................................................. 180

Appendix B - Interview responses ...................................................... 182

Appendix C - Creating word clouds ..................................................... 193

Appendix D - Medium density housing precedents ............................... 197

Appendix E - WCC community space precedents ................................. 214 


\section{List of Figures}

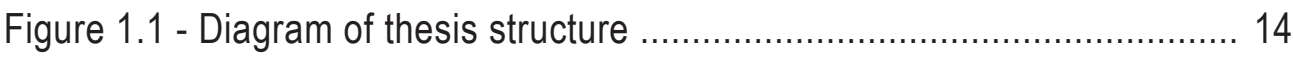

Figure 2.1 - Chronology of participation in development: a simplified history ... 24

Figure 2.2 - 'The three pillars of PRA,' by Robert Chambers - a key exponent of the approach (Adapted from Figure 6.1 Chambers 1997:105) .......... 26

Figure 2.3 - 'The Essence of Participation' - cartoon by author ...................... 29

Figure 2.4 - Arnstein's 'Ladder of Participation' (Source: Arnstein 1969)........... 31

Figure 2.5 - IAP2's Spectrum of Participation (2007) (c) International Association for Public Participation www.iap2.org ............................................... 33

Figure 3.1 - Map showing approximate locations of the majority of WCC's hous ing complexes. Two clusters around Te Aro and Newtown are evident . 41

Figure 3.2 - Three broad phases to social housing, felt globally (Source: HSAG

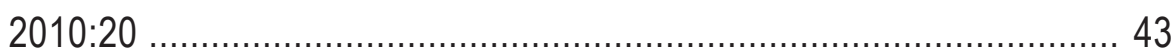

Figure 3.3 - Percentage of total 'housing need' met by existing social housing units in Wellington City (Data from DTZ NZ and Stimpson \& Co 2006:13)

Figure 3.4 - Diverse consultant group engaged in initial concept design report for Te Ara Hou. Cooperation represents a participatory process in itself ... 47

Figure 3.5 - Diagram of WCC's Existing Approach 49

Figure 3.6 - Pre-upgrade Walk and Talk session at Te Ara Hou to discuss aspects of existing environment ........................................................ 50

Figure 3.7 - Activate 1 (Nov 2008). Groups discussed themes around tables (general, exterior, interior, landscaping) 50

Figure 3.8 - Activate 1 (Nov 2008). Groups reported back findings to wider group 51

Figure 3. - Activate 2 (Feb 2009). Consultants presented a more developed design to tenants 51

Figure 4.1 - 'Walking and Talking' in Mashimoni ......................................... 55

Figure 4.2 - 'Walking and Talking' in Mashimoni .......................................... 55

Figure 4.3 - 'Dreaming through drawing' exercises ....................................... 56

Figure 4.4 - 'Dreaming through modelling' exercises ................................... 57

Figure 4.5 - 'Dreaming through typologies' exercise ..................................... 60

Figure 4.6 - Diagram of ASF Workshop's process ........................................ 61

Figure 4.6 - Kit of parts' for the portfolio of options exercise [extracted from

Figure 162 of the Change by Design report (French 2011:149)] 62

Figure 4.7 - Voting on a particularly contentious element of the portfolio of op tions exercise 
Figure 4.8 - Facilitating a 'portfolio of options' exercise

Figure 4.9 - Participants discussing housing typologies in the "portfolio of options' exercise 65

Figure 4.10 - One end result from a 'portfolio of options' exercise 65

Figure 4.11 - 'Tiles' of housing types (row, tower, etc) allowed for quick calcula tions of how many people a proposal would house .............................. 68

Figure 5.1 - Evening Post article dated 13.03.74 …..................................... 75

Figure 5.2 - Block entrance pre-upgrade ............................................... 76

Figure 5.3 - Grim outdoor space prior to upgrade ........................................ 77

Figure 5.4 - Site Plan of Te Ara Hou (N.T.S) ................................................. 77

Figure 5.5 - Re-worked outdoor space following upgrade .............................. 78

Figure 5.6 - Graph showing Te Ara Hou's proportionately high number of two and three bedroom units compared with WCC's portfolio as a whole .......... 78

Figure 5.7 - Entrance off Daniel Street post-upgrade .................................... 79

Figure 5.8 - Playground on corner of Constable and Daniel Streets also redevel oped with upgrade ............................................................... 79

Figure 5.9 - Posters advertising workshops were pinned up around complex .. 93 Figure 5.10 - Tokens identified participants' favourite and least favourite areas on the site model. Photo used with permission of participants 94

Figure 5.11 - One possibility of infill development (white foam) on the site ex plored in a workshop. Photo used with permission of participants ....... 94

Figure 5.13 - Examples of favourite and least-favourite areas around the com plex 95

Figure 5.14 - 'Discussions over drawings' - Aerial for overview of 'proposals'

(before - top; after - bottom). Annotated with a tenant's response ........ 98

Figure 5.15 - 'Discussions over drawings' - proposal to add levels to crèche (before - top; after - bottom). Annotated with a selection of responses. 99

Figure 5.16 - 'Discussions over drawings' - proposal to add levels to crèche (before - top; after - bottom) 100

Figure 5.17 - 'Discussions over drawings' - proposal to add levels to crèche (before - top; after - bottom). Annotated with a response from a tenant.101

Figure 5.18 - 'Discussions over drawings' - proposal of South-east building

(before - top; after - bottom). Annotated with responses from tenants .. 102

Figure 5.19 - 'Discussions over drawings' - proposal of North-east building

(before - top; after - bottom). Annotated with a response from a tenant.103

Figure 5.20 - 'Discussions over drawings' - proposal of North-east building (be

fore - top; after - bottom). Annotated with a response from a tenant .... 104

Figure 5.21 - A well-subscribed workshop in Kenya - we had buy-in 107 
Figure 5.22 - Site Plan showing locations of two components of proposal ....... 121

Figure 5.23 - Photograph from inside complex looking to car parking area ...... 122

Figure 5.24 - Photograph looking westward along Constable Street ............... 123

Figure 5.25 - Existing Site Plan. Scale 1:100 …......................................... 125

Figure 5.26 - Proposed Ground Floor Plan. Scale 1:100 ............................... 127

Figure 5.27 - Proposed First Floor Plan. Scale 1:100 .................................. 128

Figure 5.28 - Proposed Second Floor Plan. Scale 1:100 .............................. 129

Figure 5.29 - Rear view ..................................................................... 129

Figure 5.30 - View along Constable St ................................................ 131

Figure 5.31 - View across Constable St .................................................... 132

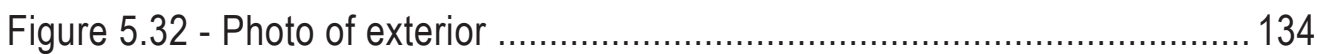

Figure 5.33 - Photograph of interior ............................................................ 135

Figure 5.34 - Existing Floor Plan. Scale 1:50 ............................................ 137

Figure 5.35 - Proposed Floor Plan. Scale 1:50 ............................................ 139

Figure 5.36 - Section through room ....................................................... 140

Figure 5.37 - Designing a cross-programmed small space: fold-down desks ... 141

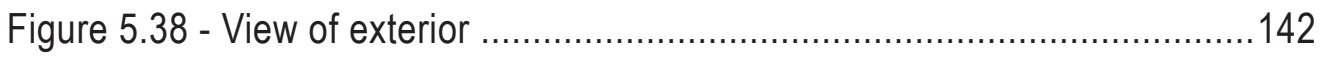

Figure 6.1: Site Plan (N.T.S) of Arlington Apartments showing possible infill op portunities 159

Figure 6.2: Symposium at UN-HABITAT (Kenya) to develop collective thought from a range of stakeholders ............................................................ 161

Figure 7.1: 'Negotiating a shared image' (Source: Zeisel 2006:50) ................ 166 


\subsection{Introduction}

\subsection{Introduction}

Participatory design takes the generally expert-centric world of design and opens it up to lay people. It is the means through which lay people, be they future residents of a development or the general public who occasionally use or pass by a development, are engaged in a design process. Design ultimately serves people, and participatory design stands for the inclusion of those people it serves through all design phases. It is in this fascinating collision of the typically expert-centric world of design with the rich and messy world of regular people that this thesis finds its subject.

This research explores current theory and practice of participatory design both in New Zealand and abroad. The study reviews literature on participation, then looks at two example participatory processes - that employed by Wellington City Council's (WCC's) in their current Housing Upgrade Programme, and that facilitated by Architecture Sans Frontieres (ASF) in their participatory slum upgrading workshop in Nairobi, Kenya. It then tests some participatory exercises first hand, informed by findings from both the literature and also a comparison between the abovementioned processes. The study hypothesises that a comparison of these two seemingly disparate sites for participation, together with a review of literature, can offer unique insights that could inform a more effective participatory programme in WCC's Housing Upgrade.

The research aims to offer applicable suggestions on how WCC could develop their participatory approach, which is timely as 16 years of the 20 -year Housing Upgrade Programme are yet to be carried out so there is significant potential to influence some of that work in some relatively minor way. Also, comparing participatory processes in the developed world with those in the developing world gives the study a certain distinctiveness. Much research is done on participation in developed countries (e.g. Warburton 2005; Wates 2008; Planning Aid England 2010) and likewise in developing countries (e.g. Fisher 2001; Cooke 2004; French 2011), however rarely is a bridge between the 'two worlds' looked to for direction. 


\subsection{Introduction}

\subsection{Literature Review}

3.0 WCC Housing Upgrade Programme

4.0 ASF Kenya Workshop

A COMPARATIVE ANALYSIS OF TWO PROCESSES

5.0 A Design Experiment

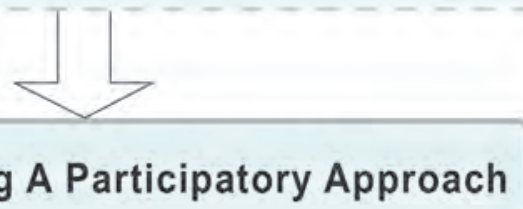

6.0 Developing A Participatory Approach

Figure 1.1 - Diagram of thesis structure

7.0 Overall Reflections 


\subsection{Thesis Structure}

This thesis consists of seven chapters and includes some graphic material illustrating the research process.

Chapter two is a review of the literature on participatory design and development. A broad historical overview of the topic is provided then some key elements of participation are identfied in an attempt to define participation. Material reviewed has ranged from seminal books by prominent authors on participation, to more up to date compilations of papers to journal articles. In essence, this chapter seeks to establish what might constitute 'best practice' participatory design.

Branching out from the literature, chapters three and four then offer a comparative anaylsis between two example participatory processes - in a social housing upgrading context (Wellington) and a slum upgrading context (Kenya).

The first process of the comparative analysis to be discussed is Wellington City Council's Housing Upgrade Programme (chapter three). WCC's upgrade programme is discussed at large then some wider context of social housing in New Zealand (its history and future) is provided. Following that, WCC's participatory process is examined with particular focus on the ways in which it has engaged tenants.

Chapter four introduces the other participatory process of the comparative analysis - that by Architecture Sans Frontieres (ASF) in a slum upgrading workshop I took part in in Nairobi, Kenya in June 2011. This chapter focuses on the participatory tools and techniques employed at the workshop. It then reflects on how this example process relates (or doesn't relate) to WCC's process. The comparitive analysis draws useful insights.

Chapter five continues the exploration into participatory process with 'a design experiment.' Firstly, the case site for the study - Te Ara Hou Apartments in Newtown, Wellington - is introduced. Then, tests of certain participatory exercices are documented. These tests are sparked by ideas from the comparison of the two processes (WCC and ASF), and are to determine first hand what approaches work well in this un-tested context and which do not. Following this, a more conventional 'expert'-centric process is carried out to propose a physical intervention of a community space and a small number of new housing units. This step is to 
explore how a more conventional design process slots into a broader participatory programme.

Chapter six - 'developing a participatory approach' - is a culmination of all previous chapters. It builds on all that has been learnt about 'ideal' participation and proposes 8 principles that could be used to guide the development of good participatory processes. Application of these principles is illustrated through a diagramming exercise. Three cases are profiled; WCC's existing partcipatory process is broadly assessed, as is ASF's Kenya workshop process and, thirdly, broad suggestions are made as to how the principles could inform a developed process at Arlington Apartments - another of WCC's housing complexes.

The seventh and final chapter reflects on the study as a whole. This chapter is frank, addressing successes and shortcomings of the research openly. Further areas for research are also suggested. 


\subsection{Research Definitions, Scope and Questions}

Definitions of terms are explained in more detail at the beginning of chapter 2, however for the sake of sufficiently introducing the project as a whole I outline some brief definitions here. 'Participatory design' is the process of design carried out in an inclusive way. The co-operation of a wide range of project stakeholders is core to participatory design. A 'participatory approach' to any given development challenge is the process or strategy the instigators of a development employ to engage any given set of stakeholders. The breadth of a participatory approach depends on circumstances specific to the development, but is usually made up of a wide range of methods from, for example, open days to interviews to focus group sessions.

The scope of the research is restricted to tenant participation in WCC's Housing Upgrade Programme (HUP). Other stakeholders - for example residents/businesses surrounding a development, the general public, agencies/organisations that will be involved in the use of a development - should be equally involved in a participatory process, however this study looks explicitly at only the tenant stakeholder group so that its scope is more manageable. In terms of physical sites for participation the project casts its net wide to catch influences from disparate contexts but, in the end, it is the participatory approach of WCC's Housing Upgrade that is the ultimate subject of this project's findings. Findings from the project could be more widely applicable than just WCC's HUP (through the application of 'generic principles' outlined in 6.1), however producing these wider effects is not the study's focus.

Physical, architectural intervention is explored through the study as a means through which a participatory approach can be tested. Although architectural intervention is proposed later in the study, the overall outcome of the research is to produce a revised participatory approach for tenant engagement in the Housing Upgrade Programme. This approach is informed by all steps of the thesis that preceed chapter six. The following questions are asked by the thesis in pursuit of this objective:

Lead question:

- Is there scope for widening tenant participation in WCC's Housing Upgrade Programme? 
Subsidiary questions:

- What are the fundamentals of 'best practice' participatory design?

- How can a participatory model from the developing world be used to inform and improve practices here in Wellington?

- What role do tenants currently play in the design process of WCC's HUP?

- How do bottom-up design processes interact with more top-down, professional-centric design processes? 


\subsection{Research Methodology}

\subsubsection{Action Research}

This thesis is grounded in the somewhat abstract tradition of 'action research.' Action research is is a name given to a particular way of researching one's own learning. Action research "seeks to bring together action and reflection, theory and practice, in participation with others, in the pursuit of practical solutions to issues of pressing concern to people" (Reason and Bradbury 2006:1). Core to its mission is to work towards practical outcomes in unison with the creation of new forms of understanding, "since action without reflection and understanding is blind, just as theory without action is meaningless" (Reason and Bradbury 2006:2). Ellis and Kiely reinforce this, stating "the relationship between improved knowledge through action and improved action through reflection is the main thrust of action inquiry strategies" (2005:85).

It is inextricably linked to participatory design, as "the idea of self-reflection is central [to action research]. In traditional (empirical) forms of research researchers do research on other people. In action research researchers do research on themselves in company with other people" (McNiff and Whitehead 2002:15). 'Participatory design' is discussed in more detail in the following chapter.

This research project has been largely based in a 'learn-by-doing,' experimental and self-reflective mode of working, and the field of action research has provided an appropriately loose framework within which I have undertaken the research.

\subsubsection{Case Study Approach}

This study utilises a case study research strategy at various stages. Firstly a comparison between two case-study processes (HUP and ASF) is my key methodological precedent. Later I engaged in more case study research in as I profile four WCC community spaces, and 6 precedents of quality medium density housing $(5.3 .3 ; 5.3 .4)$.

"The case study is a research strategy which focuses on understanding the dynamics present within single settings. ... Case studies typically combine data collection methods such as archives, interviews, questionnaires, and observations. The evidence may be qualitative (e.g. words), quantitative (e.g. numbers), 
or both" (Eisenhardt 2002:9).

The comparison of the two case-study proccesses involved the 'data collection methods' of observation and experience in the case of the Kenya workshop, as I was taking part first hand, and interviews and archival research in the case of WCC's process, as I learnt about the process retrospectively. Analysis of four WCC community rooms and the medium density housing precedents was more systematic as, in both instances, I had multiple cases to compare. One important method employed in case study analyses is searching for cross-case patterns. Eisenhardt suggests to "select categories or dimensions, and then look for withingroup similarities coupled with inter-group differences" (2002:18). The overall idea with rigorous cross-case searching is to force oneself to go beyond initial impressions of specific cases, and I have endeavoured to do this through this project.

\subsubsection{Closing thoughts on methodology}

Undertaking action research at a WCC housing complex has meant the project has been limited, to a degree, by the control WCC has exercised on research scope. In order for me to undertake this research - which has involved access to WCC project files and prolonged access to sites to engage tenants themselves - a formal research agreement had to be struck with WCC. This agreement controlled the project to a degree, and meant the project was not able to take place at its preferred site (this limitation is discussed more in 5.1.1).

In all - in the spirit of action research - my research methodology favoured simply 'learning-by-doing' over theoretical preparation or academically rigorous development of methodologies. This attitude seems endorsed by Huberman and Miles, who don't think that "good qualitative analysis necessarily calls for formal prerequisites (e.g. long ethnographic experience, knowledge of scientific logic, deep background in epistemology) ... Working without them, at least initially, ... can help you to see what else you need to know to do good analysis" (2002:395). Another thought from Huberman and Miles' at the close of their book reduces qualitative research to its essence, and is a useful place to conclude this chapter:

Doing qualitative analysis means living for as long as possible with [qualitative data's] complexity and ambiguity, coming to terms with it, and passing on your conclusions to the reader in a form that clarifies and deepens understanding (2002:394). 


\subsection{Literature Review}

\subsection{Introduction}

The objective of this chapter is to establish some best practice principles of participatory design. It is informed by a broad review of literature.

In essence, participation is "the action or an act of taking part with others (in an action or matter). Participation in an architectural or development context, therefore, simply means that a wide group of people are 'taking part with each other' in a development process. Paul Jenkins offers the following simplification of who the three groups of people taking part with each other are (other than the architect and other design professionals): "the client who commissions the building, the users of the building and the general public who are exposed to the building in some form or another" (Jenkins:13). As participation of the client is assumed as normal, "as virtually all architecture has some form of client" (Jenkins:13), participation in an architectural context generally refers the widening of a design process beyond 'the client' into the realms of building users and the general public. Madge offered a variant on a similar definition of participatory design in the late 1960's as he/she stated, "designers have two clients: those who pay for what is built and those who use [what is built]" (Madge 1968). Madge simply describes participatory processes as widening design control beyond those who pay for what is built to also include those who use what is built. Whatever variant on the definition is preferred, participatory development is the co-operation - that is the process of working together to the same end - of a wide range of stakeholders in a development process. It is from this most simple conception of what participation is that I sought to understand current thinking and topical issues through my review of literature.

Now, a brief elaboration on the term 'design'. The broad topic of 'participatory development' is researched through this literature review, although topic of this thesis is more specifically - and more architecturally - 'participatory design.' As becomes clear in later chapters, the two largely overlap. Throughout this thesis, 'design' (the verb) is preferred over 'Design' (the noun) and the process of design is considered firmly within a wider context of development. Zeisel's broadening of the term 'design' suits this definition; "farmers design when they figure out where and when to plant various crops. Lawyers design when they prepare a strategy for a client's defence" (Zeisel 2006:32). "Everyone designs who devises courses of action aimed at changing existing situations into preferred ones" (Simon 1969:55). 
As far as this thesis is concerned design is an inclusive (not exclusive) activity that is not limited only to spatial exploration.

This chapter now outlines a brief genealogy of participation in development that tracks 'the movement' chronologically (section 2.2). Findings from the literature review are then shuffled into chapter 2.3 - 'common themes'. This section attempts to draw forth the salient points of 'participation in development' as a reference point for the remainder of the thesis.

\subsection{A brief historical overview of participation}

"Participation has a longer and more varied genealogy in development thinking and practice than is usually acknowledged, and has been periodically regenerated around new schools of thought, institutional agendas and changing political circumstances" (Hickey and Mohan 2004:5).

Attempting to bring some order to a history littered with jargon and reincarnations, Hickey and Mohan offer a 'selective history' that maps the development of participatory theory and practice from the 1940's to today (2004:6). Chambers, on the other hand, offers a more loose reflection on the genealogy of the participatory 'movement' or 'approach,' suggesting "there is no linear progression, but rather a continuous process of experiencing, questioning, learning and sharing, of streams flowing separating, merging and spreading into new domains" (1997:209). Regardless of how rigid one makes out the boundaries between various iterations of participation to be, it is important to understand the context of where 'participation' has come from. This brief historical overview is by no means an exhaustive genealogy but it provides a quick insight drawn from the literature on the historical trajectory of participation and provides some base-level understanding of this trajectory that is useful in interpreting the remainder of the study. 


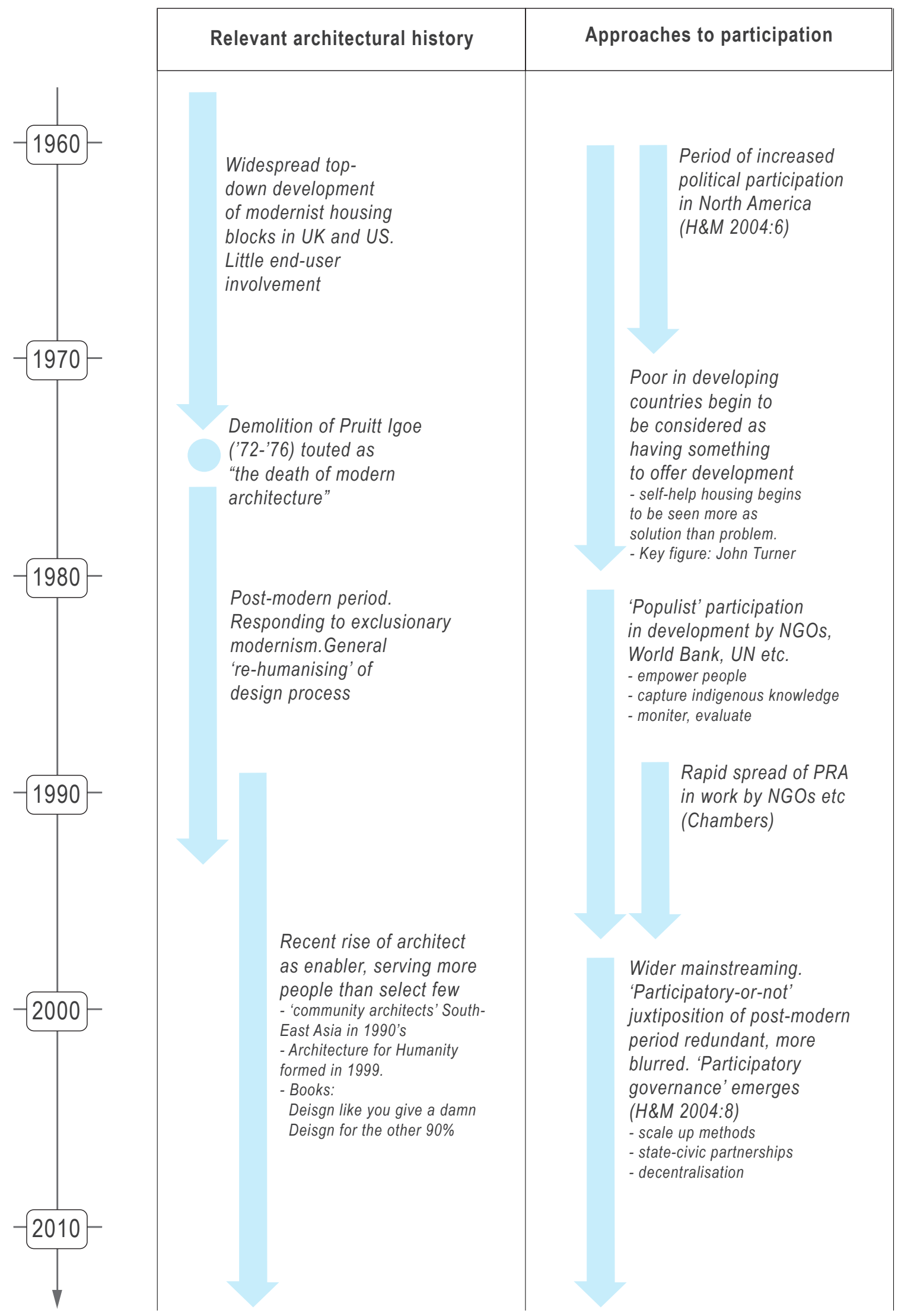

Figure 2.1 - Chronology of participation in development: a simplified history 


\subsubsection{Participation - a long and varied history}

The first point is that although 'participation' has evolved through various phases and experienced times of resurgence in the last 50 years, it has a far longer history than that. A key figure in contemporary thought on participation, Henry Sanoff, points out that citizen participation in community decision making can be traced as far back as Plato's republic written in 380BC. "Plato's concepts of freedom of speech, assembly, voting, and equal representation have evolved through the years" (Sanoff 2008:57). With that long history in mind, it is useful to mention a more recent guise that exemplifies the cross-disciplinary nature of 'participation.' A key stimulus for participatory design in northern Europe in the early 1970's emerged from the IT sector as computer professionals, union leaders and members of the Iron and Metalworkers Union "strove to enable workers to have more influence on the introduction of computer systems in the workplace" (Sanoff 2008:58). Chambers' analogy of a braided stream participatory design separating, merging and 'spreading into new domains' like a braided stream is proven by this example. Although this thesis focuses on participatory design as it relates to the development of the built environment, it is important to note its cross-disciplinary nature.

\subsubsection{Participation through pivotal post-modern period}

Looking more specifically now at participation in developing the built environment, the post-modern period emerges as pivotal. It was a reaction to top-down design process that had very much lost its way through the modernist period that stimulated a surge in participatory thinking and action in the late 1960's. A key informant of the populist approaches to participatory development practiced today is this reactionary, post-modern period. This surge occurred amongst a wider culture of general 're-humanising' of the design process that was typical of the period (e.g. Jacobs 1961). Hickey and Mahon describe this emerging approach:

It asserted the importance of placing local realities at the heart of development interventions and the need to transform agents of development from being directive 'experts' to 'facilitators' of the collection of local knowledge and the 'enablers' of capabilities (2004:11).

Although this approach sounds agreeable in theory, it came under increasing criticism through the 1990's due to its over-simplification of the realities of development processes and "a tendency for certain agents of participatory development 
- Hand over the stick

- 'They can do it'

- Use your ono best judgement at all times

- sit down, listen, learn, respect

- Unlearn

- Relax

- Embrace ever

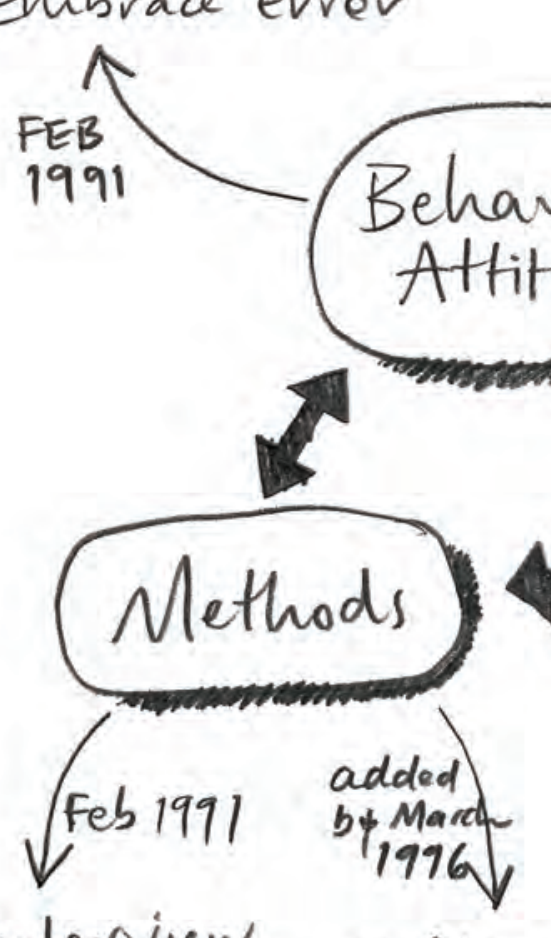

- Interview

- Map

- Model

- Rank

- Score

- Analyse

- Diagram

- Present

- Plan
- Observe

- List

- Compare

- count

- Estimate

- Act

- Monster

- Evaluate
- Facilitate

- Donor rush

- Ask them

- Have fun

- Be nice to people Added by
March 1996

Sharing

- They shave their knowledge and analysis with each other and with us

- All shave experiences of living, food...

- Organisations, trainers share their training camps, experience, wi others - NaOs, Government, Donors, Universities...

- Partnership.

Figure 2.2 - 'The three pillars of PRA,' by Robert Chambers - a key exponent of the approach. (Adapted from Figure 6.1 (Chambers 1997:105).

28 
to treat participation as a technical method of project work rather than as a political methodology of empowerment" (Hickey and Mohan, 11). Negative critique of this populist guise of participation is perhaps most strongly represented by Cooke and Kothari's book 'Participation: The New Tyranny?' Debates both for and against participation were gathering momentum at the turn of the 1990's.

\subsubsection{Participatory Rural Appraisal - an important chapter}

Another important chapter in the genealogy of participation is the rapid spread of PRA through the international development world in the first half of the 1990's. Although primarily based in the most impoverished parts of the world, it is relevant to a broader discussion as it is such a key thread of the mainstreaming of participation. Participatory Rural Appraisal (PRA) is a "family of approaches and methods to enable local people to share, enhance and analyse their knowledge of life and conditions, and to plan, act, monitor and evaluate" (Chambers 1997:102) (see Figure 2.2). Its premise is that an actively involved and empowered local population is essential to community development. This 'menu of methods' is diverse, but includes focus group sessions, social mapping, preparation of visual timelines, etc. Its critics argue that it doesn't work, it is overly intrusive of the poor and vulnerable's secrets (Cooke and Kothari 2001), with wealth ranking a particularly controversial exercise in the PRA toolbox. Its proponents, on the other hand, argue that it is simply more appropriate than top-down development methods gone before it. "As professionals have become more aware of errors and myths, and of the misfit between the reality they construct and the reality others experience, some have sought and developed new approaches and methods in their work" (Chambers 1997:102). Over time, PRA has developed from stressing methods to stressing behaviour and attitudes of facilitators. See Figure 2.2 for the three pillars of PRA.

\subsubsection{Wider mainstreaming of participation to today}

At its peak, PRA was advocated for simply as it presented a way forward that was better than the 'non-participatory' methods of development that had gone before it. Today, however, the contours of debates over participation have become more complex:

The importance of participation in development can no longer juxtapose the alleged benefits of bottom-up, people-centered, process orientated and 'alternative' approaches with top-down, technocratic, blueprint planning of state-led modernisation" (Hickey and Mohan 2004:4) 
It is the mainstreaming of participation, they claim, that has made the clean cut, 'either-or' juxtaposition redundant, or at least 'helped to blur those neat divisions.' In other words, with the norm today for participation to occur in development programmes, the question simply becomes 'how well does it occur?' This is not to say that 'top-down, technocratic, blueprint planning' is extinct - it is just less common to occur in its pure form. It could be said that if pure non-participatory processes were the opponent of good participatory practice in the post-modern period, it is the array of tokenist, misguided participatory approaches that are today's opponents of good practice. In all, and regardless of today's challenges, the mainstreaming of participation could generally be considered a positive evolution of practice that has occurred over the last 50 years.

\subsubsection{Interpretations and implications for research}

By conducting this historical overview of participation, I have developed a more mature understanding of the wider framework within which today's practice of participation sits. A key realisation for me is the more blurred boundary between an 'un-participatory' programme of development versus a 'participatory' one. It becomes clear in assessing WCC's programme (chapter six) that positive participation is certainly occurring and, in many ways, to very positive effect. WCC's participatory approach will be assessed in a more balanced way, in light of the fact that the recent mainstreaming of participation has made the contours of debate far less clean-cut than they were in the 1970's or 80's.

An understanding of PRA methods (and, more broadly, 'populist' participatory methods used in the developing world from the 1980's) gives useful context to the Kenya workshop discussed in chapter four, as the workshop methodology broadly follows this tradition. Understanding of methods like PRA from this review of literature, coupled with first hand experience in Kenya will provide a solid foundation upon which these methods can be compared with those employed by WCC.

I close this sun-chapter with a light-hearted cartoon that traces participation well before even Plato's republic to the time of cavemen. It attempts to strip participation to its essence. 
The time came for the newlysettled tribe to position the fireplace?.

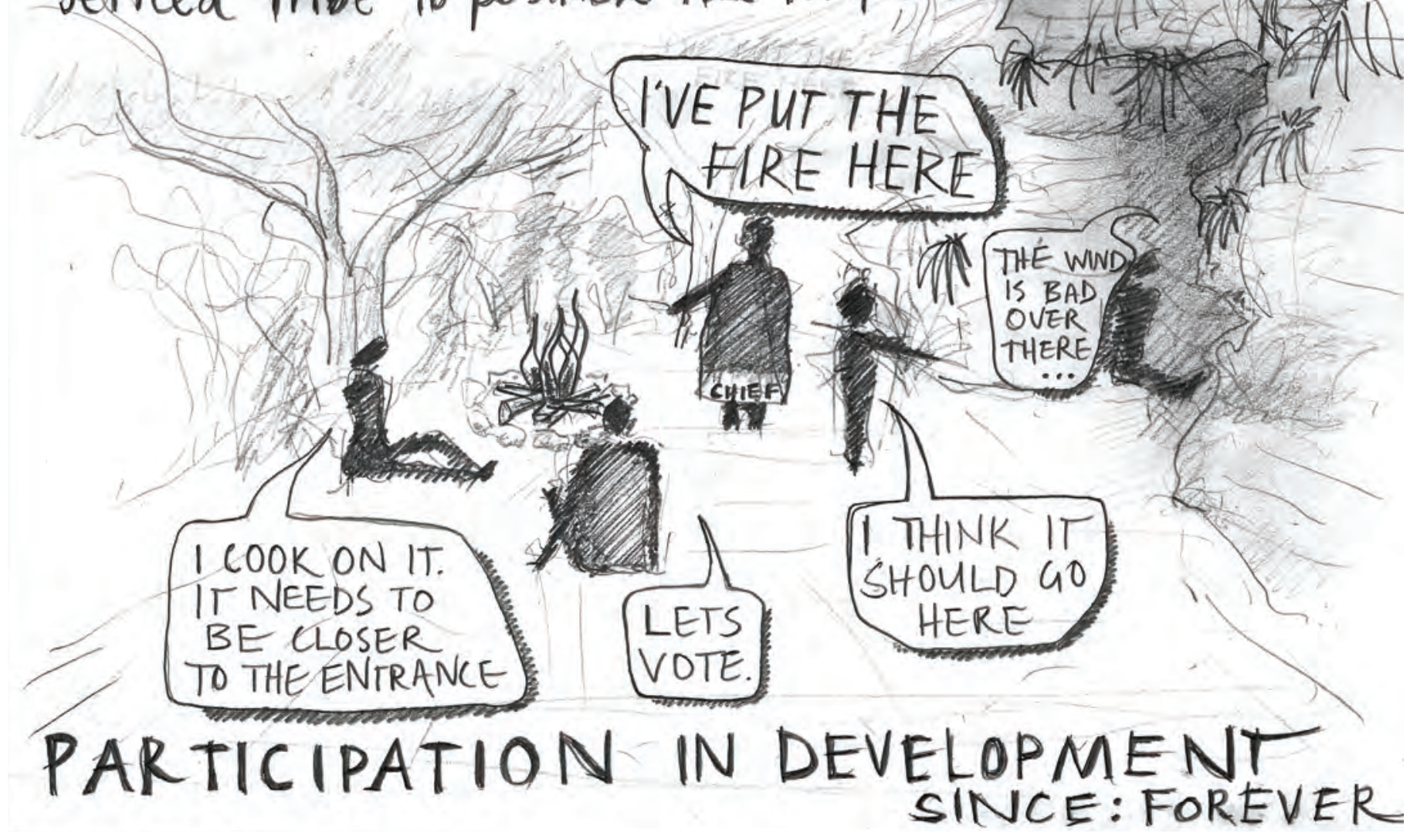

Figure 2.3 - 'The Essince of Participation' - cartoon by author.

31 


\subsection{Defining Participation}

As well as offering insights to the genealogy of participation, the broad review of literature also revealed a loose set of fundamental points common across a variety of sources that characterise participatory design as an area of thought. This section discusses those themes. This is by no means an exhaustive set of principles; simply those that seem the most pertinent to this particular thesis. This section responds to Cornwall's call: "different strands of the literature on participation offer different solutions: perhaps one starting point is to do more to bring them together?" (Cornwall 2004).

\subsubsection{Processes classified along a continuum}

Participatory processes can be classified along continua as they all differ in their extent and authenticity of public involvement. This approach of classification along a continuum is appropriate given that there is not one set way of 'doing participation,' rather a plethora of ways. One well-known early publication of such a continuum is Arnstein's activist-style 'ladder of participation' (1969) - see Figure 2.4 at right. This relates to the attitudes and intentions of participatory programmes ranging from 'manipulation' and 'therapy' (forms of non-participation) through 'tokenist' approaches like 'informing' or 'consulting,' leading to complete 'citizen control' of programmes. With her ladder, Arnstein attacks the many phony approaches to participation that were emerging as 'participation' gained currency.

[1] http://iap2.org/ Accessed 13.01 .12

[2] Wellington City Council (WCC) was guided by IAP2 in formulating its participatory approach in the Housing Upgrade Programme
Another continuum is by the International Association of Public Participation (IAP2), "the preeminent international organization advancing the practice of public participation". " IAP2 is an association representing and serving 'participation practitioner' members like local councils and large companies, generally of the developed world. ${ }^{2}$ IAP2's 'spectrum of pubic participation' offers practitioners the following gradation of methods: inform, consult, involve, collaborate, empower (see Figure 2.5). Although IAP2 state that the level of public impact increases higher up the spectrum, it does seem to advocate for each tier as a valid form of participation. This positivist approach contrast's Arnstien's ladder, and other more contemporary critics (e.g. Cooke and Kothari 2001) who insist that processes are only truly participatory when they move beyond tokenism and into the higher, power-sharing tiers. It should be noted that this spectrum is accompanied by a set of 'core values' that do encourage more genuine 'higher tier' practices (e.g. Core Value 5: "Public participation seeks input from participants in designing how they 


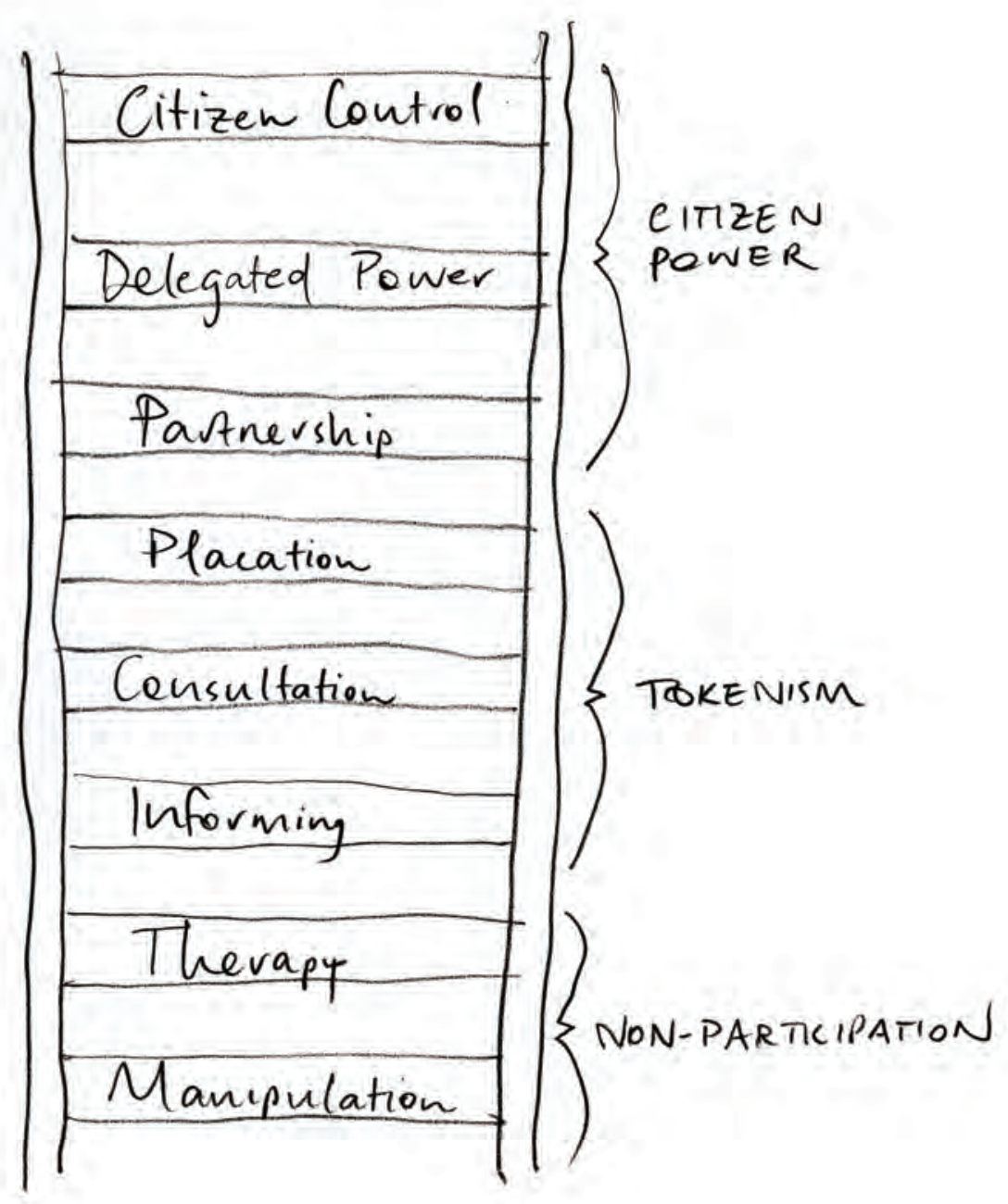

Figure 2.4 - Arnstein's Ladder of Participation (1969)

33 
[3] See IAP2's 'core values for the practice of public participation' http://www.iap2.org/displaycommon.cfm?an=4 accessed 13.01.12

participate"). ${ }^{3}$

Whether thought on participation comes from a more impartial academic realm or an association representing those engaging in its more 'populist' forms, all agree that the multiplicity of approaches to participation can generally be placed along continua ranging from little public involvement to much public involvement.

\subsubsection{Participation too often under-done}

There is a general feeling in the literature that participation is too often underdone; what often passes as participatory process is mere rhetoric. In relation to the discussion above about the spectra along which participatory processes can be placed, this point simply argues that too often processes sit 'on the lower rungs on the ladder' when they should be higher. Of the 'rise of participation' through the post-modern decades, Robert Chambers points out that "as usual with concepts, which gain currency, rhetoric has run far, far ahead of understanding, let alone practice" (Fischer 8). Fischer, too, is clear that 'real' participation is not "rudimentary consultation between project staff and community members" (10). Others acknowledge that participation in development can operate across a full spectrum of 'intensities'; indeed, responding to a generic survey about an upcoming development proposal is a form of participating, albeit in a rather passive, minimal way. However cynical commentators are towards mainstreamed, 'phoney' participation, all agree that participation in its most genuine form goes beyond mere consultation. In response to the common misconception that participation is simply another hurdle in the way of development, Planning Aid England state that "community consultation and engagement should not be carried out just to tick a box - it should have a clear purpose and be of value to all involved, otherwise it might do more harm than good" (Planning Aid England). Also encouraging readers to go beyond the box-tick approach is Nick Wates, an author of participatory manuals. His comments like "no one should waste their time - no one should feel stymied" show the unfortunately common understanding of participation that the manuals are out to prove wrong (Wates 2008:16).

\footnotetext{
"Full participation ... is not about getting people to agree to proposals drawn up by professionals; it is about creating better proposals and therefore better places. Improving quality of life becomes a shared goal" (Wates 2008:xvi)
}

Through the review of literature, it has become clear that much of the 'pro-partici- 


\section{IAP2 Spectrum}

\section{of Public Participation}

\section{Inform}

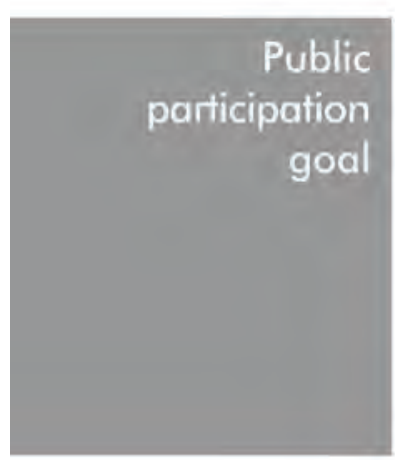

To provide the public with balanced and objective information to assist them in understanding the problem, alternatives, opportunities and/or solutions.

\section{Consult}

To obtain public feedback on

analysis,

alternatives and/or decisions.

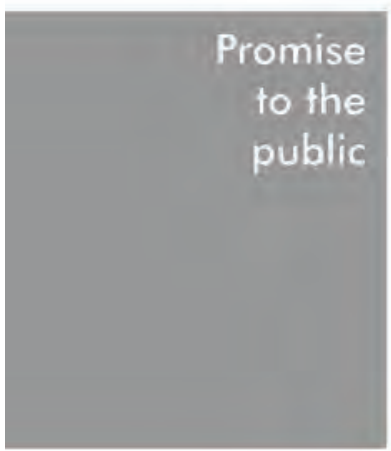

We will keep you informed.
We will keep you informed, listen to and acknowledge concerns and aspirations, and provide feedback on how public input influenced the decision.

\section{Involve}

To work directly with the public throughout the process to ensure that public concerns and aspirations are consistently understood and considered.

\section{Collaborate}

To partner with the public in each aspect of the decision including the development of alternatives and the identification of the preferred solution.
We will work with you to ensure that your concerns and aspirations are directly reflected in the alternatives developed and provide feedback on how public input influenced the decision.
We will look to you for advice and innovation in formulating solutions and incorporate your advice and recommendations into the decisions to the maximum extent possible.
We will implement what you decide,
To place final decision-making in the hands of the public.

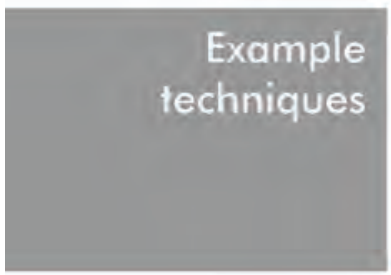

- Fact sheets

- Web sites

- Open houses
- Public comment

- Focus groups

- Surveys

- Public meetings
- Workshops

- Deliberative polling

\footnotetext{
- Citizen advisory committees

- Consensusbuilding

- Participatory decisionmaking
} 
pation' material is geared firmly in opposition to 'under-done,' box-tick approaches to participation which, one gets the impressions, are too common in contemporary development.

\subsubsection{Participation cuts across traditional boundaries}

Perhaps going some way to explain the cynicism heard from the professional establishment, Sanoff describes participatory design as a "movement that cuts across traditional professional boundaries and cultures" (2008). He spreads this sentiment into the world of research, too, stating that good participatory process should break down traditional barriers between an 'expert researcher' and a 'researched community' (2007:59). Nabeel Hamdi states this simply in a lecture, regarding state-led upgrading projects:

\footnotetext{
We have to move away from the idea that an expert alone is some special kind of person, and accept that every person out there is a special kind of expert ... I engage with [lay people] in the same way l'd engage an engineer if I was putting up a complex building. I need that tacit knowledge [lay people have about their places]. That intelligence is not in the planning office - it's in the street. I need to engage with the street as my source of knowledge. ${ }^{4}$
}

Core to the argument for increased participation is the rising of 'lay' stakeholders to 'expert' status (i.e. a resident is an expert on how their place works and what it needs in the same way that, for example, an urban designer is an expert on the elements that make up a positive public space). This is, in some ways, counterintuitive to established professional boundaries and cultures, which goes some way to explain why participation is often met with cynicism or resistance.

\subsubsection{Processes can't be neatly packaged}

Another 'fundamental' of participatory processes is that they can never be neatly packaged or predictable as they are highly context-specific (Fisher 2001:7). They resist standardisation. Chambers advises facilitators of participatory processes to be 'optimally unprepared.' 'Participatory processes cannot be 'properly planned,' where 'properly' refers to fixed content and strict timetables. Optimal unpreparedness," he points out, "liberates a facilitator ... to go with the flow, roll with the

4 Nabeel Hamdi, A Public Lecture at The Maldives National University, http://www.youtube.com/watch?v=gfChmfcrt38\&feature=player_embedded accessed 17.08.11 
punches, and steer by sailing and tacking with the wind. Good workshops are more like a sea voyage than putting up a building" (2002:xiv). Interestingly, this strategy - or 'non-strategy' - sits well amongst general thought on conducting qualitative research, as Huberman and Miles point out "the core requisites for qualitative analysis seem to be a little creativity, systematic doggedness, some good conceptual sensibilities, and cognitive flexibility - the capacity to rapidly undo your way of construing or transforming the data and to try another, more promising tack" (2002:394). A keen responsiveness to changing circumstances and a willingness to endure a messy process and seem vital to the management of good participatory processes. This flexibility inherent in the process certainly does not mean there isn't a need for processes to be well thought out. On the contrary, good processes, for all their flexibility, do require a strong skill set to be well-run. "There is no "perfect recipe" for good participatory process, as circumstances will always be different, however there certainly are good practice "ingredients" that must be of a high standard to produce a good outcome (Planning Aid England).

Just as it is difficult to neatly package or predict how to manage participatory processes, it is also difficult to assess and neatly report on the exact effects of a participatory process. In her article, Is participation too expensive?', Warburton admits "it may not be possible to demonstrate direct cause and effect, especially as participation is so often just one element of a larger programme (for example alongside investment in physical regeneration)" (2005:316). Furthermore, effects of participation are often more social than physical, anyway; "experiences in the participation process show that the main source of user satisfaction is not the degree to which a person's needs have been met, but the feeling of having influenced the decisions" (Sanoff 2008:67). This sense of involvement, again, becomes difficult to assess or 'package', but most would agree is a worthy outcome of participatory process.

\subsubsection{Processes engage power-relations}

Participatory processes inescapably engage in power-relations. Participatory processes can redistribute power or maintain the status-quo although, as Arnstein points out, "participation without redistribution of power is an empty and frustrating process for the powerless" (Arnstein 1969). Theoretical positions on powerrelations in participatory development fall into two general camps. The first is the 'harmony model of power' (outlined in Mohan and Stokke 2000:249), in which it is argued that empowerment of the powerless has no significant negative effects 
upon the power of the powerful. This position draws strong critique from the participation as tyranny camp of Cooke and Kothari, who call for "a more sophisticated and genuinely reflexive understanding of power" (2001:14). Their position represents the second camp, agreeing with Foucault (1977) and Kesby (2003) that power-play is inescapable; that it is impossible to escape to a pure space beyond power. "We have no choice but to draw upon less dominating forms of power to destabilise and transform more dominating frameworks of power" (Kelly 2001). The context of, say, tenant participation in council housing upgrades sits more comfortably in this second camp, given the landlord-tenant power relations at play in this context. A 'harmony model of power' between council and tenants difficult to imagine, if not impossible, and it is more realistic to imagine council dominated power relations in this context will inevitably remain, given that they own the housing. Turning back to Kelly, 'less dominating forms of power' must be drawn upon.

This discussion on power relations reinforces an earlier point that participation cuts across traditional professional boundaries and cultures (2.2.3). Pushing through the traditional boundaries of what an architectural design process can deliver, a more participatory process can have an enduring impact on how power relations develop in a given site. One example of this is that participants' own capacity to instigate changes themselves can be built through a process (examples of this occurring throughout the ASF workshop in Kenya are discussed in 4.4.4). As mentioned above, however, the empowerment of one group can be threatening for those holding power in an existing structure. Key to negotiating this potential tension is the need for all parties to participate in a process under the understanding that outcomes can be win-win for all involved. It is important to bear power relations in mind when reviewing or taking part in a participatory process.

\subsubsection{Participation cultivates choice and flexibility}

It is often through good participatory processes that built outcomes can best meet the diversity of the people they serve. Hamdi states that participatory processes should cultivate choice and flexibility in outcomes (2010). "The tradition of one 'best' solution for all people does not recognize that there is a need for different solutions for different people, that different ways of life call for different types of neighborhoods" (Sanoff 2007). It is in this sense, Sanoff argues, that some of the determinist principles of New Urbanism are repeating the same mistakes of the socially-homogenising values of modernism. To counter this ill, participation reveals diversity therefore naturally resists homogeneity. Increased awareness 
of this diversity can lead to two responses. Firstly, variation can be embraced in physical interventions (e.g. one bedroom units should not be homogenous in their characteristics as occupants of those units will all vary in their preferences). Secondly, greater occupant personalisation can be allowed for in a physical intervention (e.g. designing units as shells that can be inhabited in a number of different ways). Both responses can inform how physical interventions are carried out, and are discussed following this project's physical proposal in section 5.4.3.

\subsubsection{Participation creates a collective intelligence}

Another point about participatory processes worthy of note is that they have the potential to bring into being a collective intelligence. Atlee describes this as a "shared insight that comes about through the process of group interaction, particularly where the outcome is more insightful and powerful than the sum of individual perspectives" (2003). This depends on good facilitation of a process and some common interest in a process that results in participants contributing meaningfully and genuinely. It requires all participants to be seen as 'expert' contributors in their own right (see 2.3.3) in order for the collective intelligence of the whole to be maximised.

In summary, the loose set of elements defining participation as identified through the literature review are as follows: Participatory processes -

- can be classified along continua

- are too often 'under-done'

- cut across traditional professional boundaries and cultures

- can never be neatly packaged or predictable

- inescapably engage in power relations.

- cultivate choice and flexibility in outcomes.

- bring into being a collective intelligence. 


\subsubsection{Interpretations and implications for research}

Preparation of the above definition of participation has created a solid foundation for the remainder of the study in three main ways. Firstly, I proceed through subsequent chapters with the expectation that I am not going to be able to neatly package or predict a participatory process, which helps me to 'live with the complexity and ambiguity' (Huberman and Miles 2002), or the 'messiness' of participatory research. Reviewing processes with a continuum in mind (from inform to empower) assists me in comparing participatory processes of WCC and ASF, as it provides some common criteria with which an approach can be assessed. Secondly, an understanding of the common view that participation is too often 'under-done' keeps me on the look out for inauthentic approaches attempting to be passed as participation. Thirdly, knowing to keep power relations in mind through the study and knowing that good participatory processes empower, 'cultivate choice and flexibility' and bring into being a collective intelligence helps as I assess programmes (chapters three and four) and work towards a developed approach for WCC (chapters five and six). 


\subsection{WCC Housing Upgrade}

\subsection{Introduction}

This chapter introduces Wellington City Council's Housing Upgrade Programme, which is my local case study of a participatory process, and also became my case site for my own exploratory process. An brief introduction to WCC's Housing stock and upgrade is followed by an overview of the 'history and future of social housing.' The participatory approach of WCC through the upgrade programme is then discussed, followed by a more detailed introduction of my site - Te Ara Hou complex in Newtown.

Wellington City Council is the largest provider of social housing in Wellington City, and the third largest in the country after Housing New Zealand Corporation and Christchurch City Council (Wellington Civic Trust 2006:9; DTZ NZ and Stimpson \& Co 2006:8). WCC's housing stock provides around 2300 homes for approximately 4000 tenants of diverse cultural and social backgrounds. Its tenant mix is consistently low-income but diverse in make up with, for example, $80 \%$ of tenants receiving central Government income support of some kind, only $42 \%$ of tenants belonging to the Pakeha/European ethnic group (compared with $70 \%$ across Wel-

[1] 2006 Census - 'QuickStats about Wellington City' http://www.stats. govt.nz/ensus/2006CensusHomePage/ cessed 03.03.12 lington City ${ }^{1}$ ). Further highlighting diversity, 33\% of tenants are refugees and migrants (Wellington Civic Trust 2006:11). "Wellington City entered the provision of social housing to meet a mixture of urban renewal, housing and labour shortage objectives". Its stock is generally centrally located, is relatively high density $(67 \%$ of units in tower blocks) and has a high proportion of bedsits or one bedroom units (Wellington Civic Trust 2006:9). Many of the large complexes in the portfolio were constructed during a surge in development in the 1960's when, under the guidance of the late George Porter - an architect, city councillor and chair of the then 'housing committee' -, the city took maximum advantage of central Government housing grants and low-interest loans" (Wellington Civic Trust 2006:4).

By the end of the 1990's, WCC was facing the pressing challenge of ballooning maintenance requirements of an aging portfolio of housing, To assist with this challenge, WCC secured a grant from central Government in 2007 worth $\$ 220 \mathrm{~m}$ to assist with an upgrade. This is meeting approximately half of the total upgrade cost. In 2008, having signed a Memorandum of Understanding with Government, 


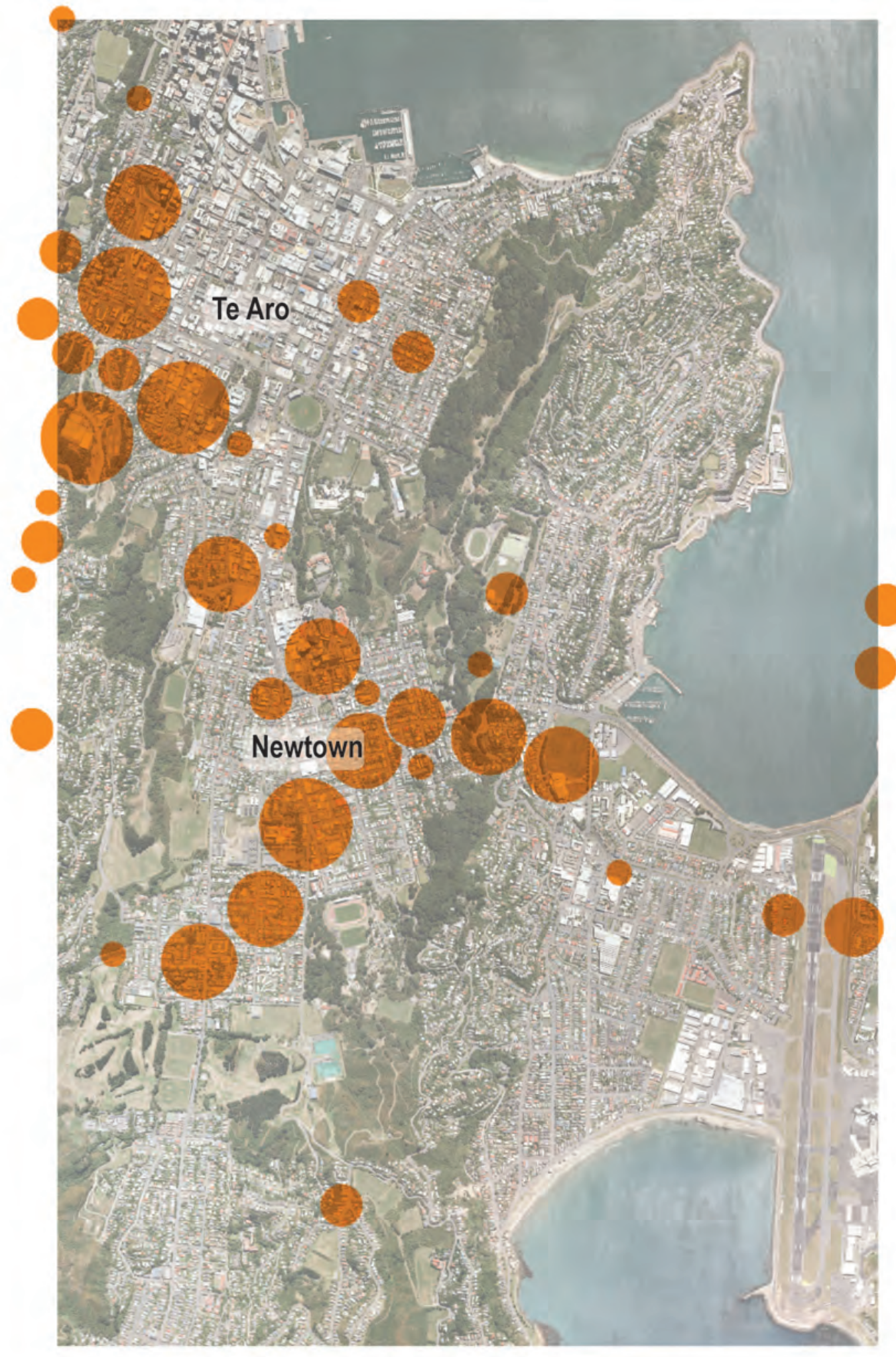

\section{Housing Complexes}

No. of units

$1-5$

$6-25$

$26-50$

$51-120$

$121-279$

Figure 3.1 - Map

showing approximate

locations of the major-

ity of WCC's housing

complexes. Two clus-

ters around Te Aro and

Newtown are evident. 
WCC began their 20-year Housing Upgrade Programme. Priorities of the programme are to bring housing up to acceptable standards of modern living (i.e. to meet thermal and seismic performance codes), improve some of the flawed planning aspects (wherever possible/feasible), improve security aspects of sites and generally 'refresh' the complexes, which have become rundown.

The programme began with a scoping exercise to identify which complexes needed priority attention. In 2012, four years into the upgrade, many of the largest complexes' upgrades have been completed or are under construction. The programme has been largely praised a great success so far, winning a 'Leading Housing Solutions' award at the 2010 New Zealand Australasian Housing Institute Professional Excellence Awards.

Accompanying the physical upgrade programme is a 'Community Action Programme (CAP) that delivers community and social development objectives. CAP's mandate includes such tasks as developing community facilities on sites and running social and recreational programmes. While linked, the Community Action team is a separate group of staff from the those managing the physical upgrade. The Community Action Programme has planned and coordinated all tenant involvement in the Housing Upgrade Programme's planning and design phases. I elaborate on this participatory programme later in 4.3 , following a broad overview of the history and future of social housing.

\subsection{History and Future of 'Social Housing'}

A brief discussion of the history and future of 'social housing' in New Zealand will help situate Wellington City Council's Housing Upgrade Programme in a broader context.

First, some clarification in terminology. This thesis is primarily concerned with the portfolio of housing owned by Wellington City Council, probably most commonly known as 'council housing.' The label 'council housing' has been superseded within Council who now refer to it as 'City Housing.' 'City Housing' is distinct from 'state housing' - that owned by Housing New Zealand Corporation (HNZC) - an arm of central government. Most broadly, the term 'social housing' refers to any housing provided with assistance for those who cannot meet their own housing needs. 'Social housing' could also be thought of as 'non-market housing,' and its providers range from central government to local government to community groups and 
private entities.

\subsubsection{Social housing through the twentieth century}

"New Zealand can look back on a century-long tradition of social housing, beginning with the Workers' Dwellings Act introduced by Richard Seddon's Liberal government in 1905" (Housing Shareholders Advisory Group (HSAG) 2010:19). Throughout the 20th Century, New Zealand broadly followed three phases of social housing policy as with the rest of the Western world. These three phases reflect global triggers like the Depression of the 1930's, World Wars and global economic crises. The three phases are summarised in Figure 3.2 below.

Specifically to New Zealand, two particular stages along this continuum are worthy

\begin{tabular}{|c|c|c|c|}
\hline & Housing the Poor & Golden Age & Reform \\
\hline Time Period & 1800 s to World War 1 & 1950 s- 1970 s & Post crises of 1970 s (and early 90 s) \\
\hline Target & $\begin{array}{l}\text { The most needy, typically the } \\
\text { urban poor in inner-city slums }\end{array}$ & $\begin{array}{l}\text { Broadly based, benefiting more } \\
\text { income groups, triggered by } \\
\text { returning soldiers, marriages }\end{array}$ & $\begin{array}{l}\text { Europe stays broadly targeted but } \\
\text { Commonwealth, NZ trend to } \\
\text { residual needs }\end{array}$ \\
\hline Funding & $\begin{array}{l}\text { Private finance, philanthropic } \\
\text { contributions. State } \\
\text { participation rises as C19th } \\
\text { starts }\end{array}$ & $\begin{array}{l}\text { Central, local government. } \\
\text { Subsidies are on producer } \\
\text { side, targeted at assets }\end{array}$ & $\begin{array}{l}\text { Reforms shift housing } \\
\text { responsibility and admin from } \\
\text { Government. Also a move to } \\
\text { consumer subsidies. }\end{array}$ \\
\hline $\begin{array}{l}\text { Subsidies } \\
\text { focus }\end{array}$ & $\mathrm{N} / \mathrm{a}$ & $\begin{array}{l}\text { General subsidies targeted at } \\
\text { assets eg \$x per Y houses }\end{array}$ & $\begin{array}{l}\text { Personal subsidies targeted at } \\
\text { individuals' incomes }\end{array}$ \\
\hline $\begin{array}{l}\text { Supply } \\
\text { change }\end{array}$ & Localised & $\begin{array}{l}\text { Significant supply increases - } \\
\text { "golden age" of social housing }\end{array}$ & $\begin{array}{l}\text { Dwindling new builds, more spend } \\
\text { on redevelopment and backlogged } \\
\text { maintenance }\end{array}$ \\
\hline Polit & Liberal capitalism & Welfare state after WW2 & Post welfare state \\
\hline
\end{tabular}

Figure 3.2 - Three broad phases to social housing, felt globally Source: (HSAG 2010:20)

of particular note. Mass construction of state housing around the time of the Second World War has contributed greatly New Zealand's state housing stock as we know it today. Of today's 67,000 state houses, 32,000 of them were built between 1937 and 1949, and construction continued into the 1970's (HSAG 2010:20). This 'golden age' also impacted the provision of social housing locally too, as central Government made low-cost finance available for local councils to develop housing in the 1960's and 1970's (Wellington Civic Trust 2006:9). The second stage worthy of note is the 1990's, which saw significant policy changes in New Zealand following the economic crisis of 1989 and ensuing recession and changes in Government. There was a "shift to a demand side approach [from the supply-driven approach of the 'golden age']. State house rents were raised to market rates, and the Accommodation Supplement was introduced to subsidise rent payments for those in the private sector and in state housing" (HSAG 2010:20). Generally, across the second half of the twentieth century, "the change of focus for state 
housing [in New Zealand] was from one of providing good quality housing to working families to providing subsidised rental accommodation for welfare recipients" (The Salvation Army Social Policy and Parliamentary Unit 2007:28).

"Over the last decade [to 2010], New Zealand has focused significant attention and resources on social housing. In 1999, the incoming Labour Government halted sales of state houses and instituted a programme of supply increases" (HSAG 2010:22). The Government's MoU with WCC signed in 2007, which kickstarted the Housing Upgrade Programme, was one such supply-increase measure. In essence, this MoU confirmed a financial contribution from Government of $\$ 220 \mathrm{~m}$ for an upgrade programme with the condition that WCC retains its stock of social housing at approximately the same level for at least 30 more years (WCC 2010). This condition is significant as it prevents WCC, at least in the short-medium term, from selling its housing stock on the open market, as it came close to doing in 1998 (Wellington Civic Trust 2006:2).

\subsubsection{Future initiatives and projections}

Looking ahead, the wider housing market (both in Wellington and nationally) is projected to continue in its current state of flux. It has been identified that New Zealand lags international benchmarks around third party activity and general provision and funding in the social housing sector (HSAG 2010:38). Feeling from the Housing Shareholders Advisory Group's 2010 report to the Government was that "the current [HNZC-dominated] model has run its course and significant intervention is required" (2010:39). It has suggested that many more creative avenues for management and development need to be explored like Public Private Partnerships, leveraging land assets (for example, HSAG estimates there is potentially $\$ 1$ billion of land undercapitalised in HNZC's portfolio), new housing designs and new delivery models. These avenues, the report argues, are more akin to how social housing is progressively developed overseas and New Zealand must catch up. Measures are beginning to respond to these recommendations, like the 'Social Housing Unit' set up in July 2011 within the Department of Building and Housing to work with the third (non-governmental) sector to develop, manage and generally encourage more development of social housing in New Zealand. It will "work with [third party] providers that [are] largely self-sustaining and [have] sources of

[2] http://www.stuff. co.nz/national/politics/5143756/New-unitfor-social-housing finance. It [will] provide funding, land or surplus state housing stock, or a combination of these, to support groups to develop a mature social housing sector". ${ }^{2}$ This central government policy development supports more integrated and creative development of Wellington City Council's housing stock too. The creative 
sentiment of HSAG's recommendations is echoed in the Housing Upgrade MoU between the Government and WCC, which states that "all parties will encourage new and creative ways to achieve the outcomes anticipated by this MoU" and, more specifically, "there is also an opportunity to achieve wider urban development outcomes through location and design of any new or replacement stock."3

Creative responses are certainly required given the current alarming trends in the wider housing sector. A report prepared for WCC and Housing NZ in 2006 to assist them in assessing the likely future demand of social housing in Wellington outlines increasing household costs (at a typically faster rate than household incomes), decreasing home ownership rates and increasing demand for more housing in the central wards of Wellington City largely through multi-unit and apartment dwelling types (DTZ NZ and Stimpson \& Co 2006:3). Regarding social housing specifically, the report projects that the number of households in Wellington City with 'housing needs' (defined as the cohort of households struggling most to meet their housing needs alone - i.e. falling in or close to social housing eligibility ${ }^{4}$ ) is projected to increase from 9767 to 13077 between 2006 and 2026 - an increase of 3310 households (DTZ NZ and Stimpson \& Co 2006:14). The graph below outlines the level of provision that existing social housing providers can offer with their current levels of housing stock, as a percentage of total housing need (calculated and projected to 2026).

As part of the response to this issue, more housing can be built (as per the 'Golden Age') or households can be targeted with more subsidies (as per the 'Reform' years). Most alarming in this graph is the gap represented by the blue arrow - if we were to assume the 'Golden Age' response of building more hous-
[3] Memorandum of Understanding (and related Deed of Grant) viewed in City Housing office.

[4] 'Housing need' is defined more specifically as 'financially stressed' households and those households in (or on waiting lists for) social, third sector and emergency housing. . For a full definition see (DTZ NZ and Stimpson \& Co 2006:16)

Figure 3.3 - Percentage of total 'housing need' met by existing social housing units in Wellington City (Data from DTZ NZ and Stimpson \& Co 2006:13) 
ing, 1452 more units would need to be added to social housing portfolios in Wellington City over the 20 year period to 2026 to maintain their 2006 level of support (DTZ NZ and Stimpson \& Co 2006:14). This is alarming when coupled with increasing demand for housing in Wellington across the housing sector as a whole, particularly in central areas.

Demand for new housing in Wellington City is projected to grow, and it is clear that that growth includes both ends of the housing spectrum. Development will need to include social housing - of some description - for those growing numbers of households in 'housing need'. Creative responses are required from all angles to boost portfolios of existing social housing providers, and find new avenues for future providers. It seems WCC are in an important position as the city's largest existing social housing provider and also given their centrally located assetbase.

\subsection{Participatory approach of WCC}

The current participatory approach WCC employ to engage tenants in their Housing Upgrade Programme is now profiled.

Participatory design processes incorporated into Housing Upgrade Projects offer considerable opportunities on a number of counts. The urban implications of a number of upgrades (given their prominent locations and often large scale) are such that a participatory process is an appropriate way to bring diverse stakeholder groups together. Also, given that the projects are upgrades of existing environments there is considerable opportunity to harness local knowledge from those who know the environments well - the tenants - in upgrading to make decisions as well-informed as possible. Tenant participation is also a considerable opportunity to build tenants' attachment to their place (which has an enduring effect on their ongoing protection of place) and, more socially, increase their sense of inclusion in the upgrade process. WCC's process, outlined in 4.3.2 and critiqued/discussed in more detail in chapter 6 , seizes some of these opportunities and, I believe, misses others.

As the remainder of this section discusses only tenant participation, I firstly offer a point regarding consultant participation in the upgrade process. WCC engage very diverse groups of design and building professionals through the programme, 


\section{novak+middleton}

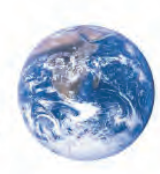

Absolutely

Positively

Wellington
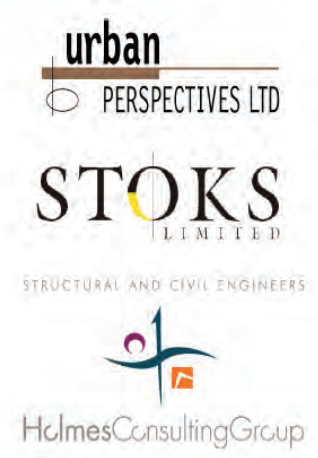

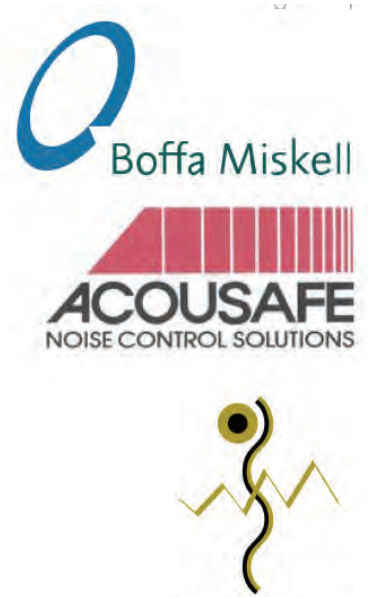

$\mathrm{h}$ ol $\mathrm{mes}$ fire \& safety

\section{STEPHENSON ARCHITECTS ANGINEERS}

Figure 3.4 - Diverse consultant group engaged in initial concept design report for Te Ara Hoi. Cooperation represents a participatory process in itself

which is of course a participatory process of sorts. There is much cooperation and negotiation between different consultants' perspectives around the table. As an example, Figure 3.4 above is a snapshot of consultants engaged in the initial concept design for all complexes. Whilst admittedly this does not carry the challenge of engaging lay stakeholders (as tenant participation does), it deserves a mention as it is, of course, a participatory aspect of the programme. The remainder of this chapter now focuses on tenant participation (herein, participation).

\subsubsection{Context of Work}

The participatory component of Wellington City Council's Housing Upgrade Programme is one very small component of a large and complex programme. Upgrade projects are carried out amongst an array of parameters and priorities that reduce scope for the upgrades from the outset, such as the need to have upgraded buildings meet building codes in areas such as insulation and seismic performance standards. ${ }^{5}$ These reasonable limitations, together with strict timeframes for projects, highlight the complex environment within which participatory components of the Housing Upgrade Programme are carried out.

In considering the participatory component of the Housing Upgrade, it is important to bear this limited project scope in mind. Where in Kenya, we ran a programme of participation towards an outcome which was not certain to eventuate (in the short to medium term, anyway), in Wellington a programme of participation has been 
[5 In some upgrades, earthquake strengthing has cost as much as one third of the total budget.
[6] As Te Ara Hou was the first complex to be upgraded in the programme, WCC admittedly worked harder on engagement at this complex than subsequent complexes. Whilst it is generally representative of the process run at other complexes, it should be considered as the most zealous of examples. designed to comfortably fit, but not bring into serious question, predefined project objectives. Established decision-making systems in the political environment of Wellington City Council limit space left for genuine bottom-up contributions from other stakeholders like tenants in key decisions around project scope and direction.

\subsubsection{Outline of Approach}

The participatory component of Wellington City Council's (WCC) Housing Upgrade Programme has been developed into a loosely standardised, repeatable model to be run at each of the city's complexes around the brief-creation stage of projects. The two main participatory components to WCC's process are pre-upgrade evaluation sessions (branded 'Walk and Talk') and tenant engagement sessions (branded 'Activate'). These two tools are carried out along with peripheral minor events and communications. By way of illustrating the process, a full outline of WCC's participatory work leading up to the upgrade of Te Ara Hou Apartments in 2009 is provided below. This should be considered generally representative of the standardised process run in subsequent upgrades ${ }^{6}$ :

- Tenant newsletters and flyers (in various languages) introduced tenants to the project and updated them throughout.

- Weekly BBQs held on site throughout process to simply to build relationships and rapport between City Housing staff and tenants.

- Pre Upgrade Evaluation sessions (branded 'Walk and Talk'). Three walkthroughs in groups of 10 or so with a built environment professional (in Te Ara Hou's case, a Post Occupancy Evaluation specialist). Participants were grouped according to their roles (e.g. Tenants, care workers, grounds maintenance workers etc).

- Tenant engagement sessions (branded 'Activate') held on three Saturday mornings with tenants in the Te Ara Hou community room. Translators were present.

1. Initial workshop (November 2008). Architect and other consultants presented initial 'concept design' in stages (general, interiors, exterior and landscaping) and participants discussed matters in groups around tables. Each group created lists of positives and negative characteristics of the their home under those categories presented. Groups also suggested 'activities and events' they would like to see happen. 30 - 35 tenants were involved.

2. Reporting back (February 2009). Architect and other consultants 
presented more developed design and got instant verbal feedback from tenants on ideas presented. This was the 'reporting back' session to show how tenants ideas had been taken on board.

3. Open day (May 2009). Architect and other consultants presented final design.

[Flyers/postcards were sent to all tenants after Activate sessions to inform them of what had happened].

The diagram below is essentially a simplified graphic representation of the information above.

\section{EVENTS}

Social events with tenants

Walk and Talk sessions

Activate - workshop

Activate - reporting back

Activate - open day

\section{KEY OUTCOMES}

Figure 3.5 - Diagram of WCC's Exisitng Approach

Figures 3.6 - 3.9 are photographs from various stages along this process. Chapter four then begins, which introduces the second subject of this comparative analysis - the ASF Kenya workshop. Discussion of that workshop (4.4) also links back to WCC's process, which completes the comparative analysis. 
Figure 3.6 - Preupgrade Walk and Talk session at Te Ara Hou to discuss aspects of existing environment

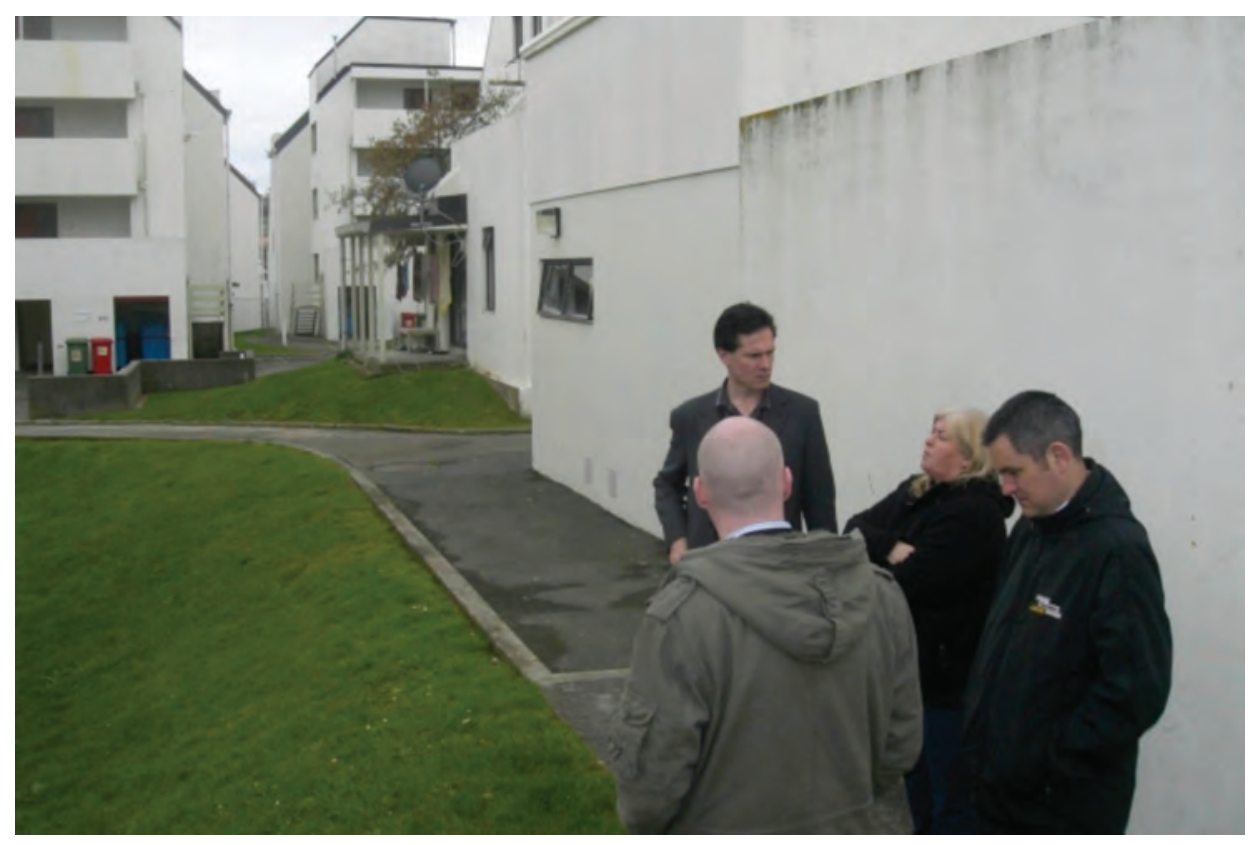

Figure 3.7 - Activate 1 (Nov 2008). Groups discussed themes around tables (general, exterior, interior, landscaping).

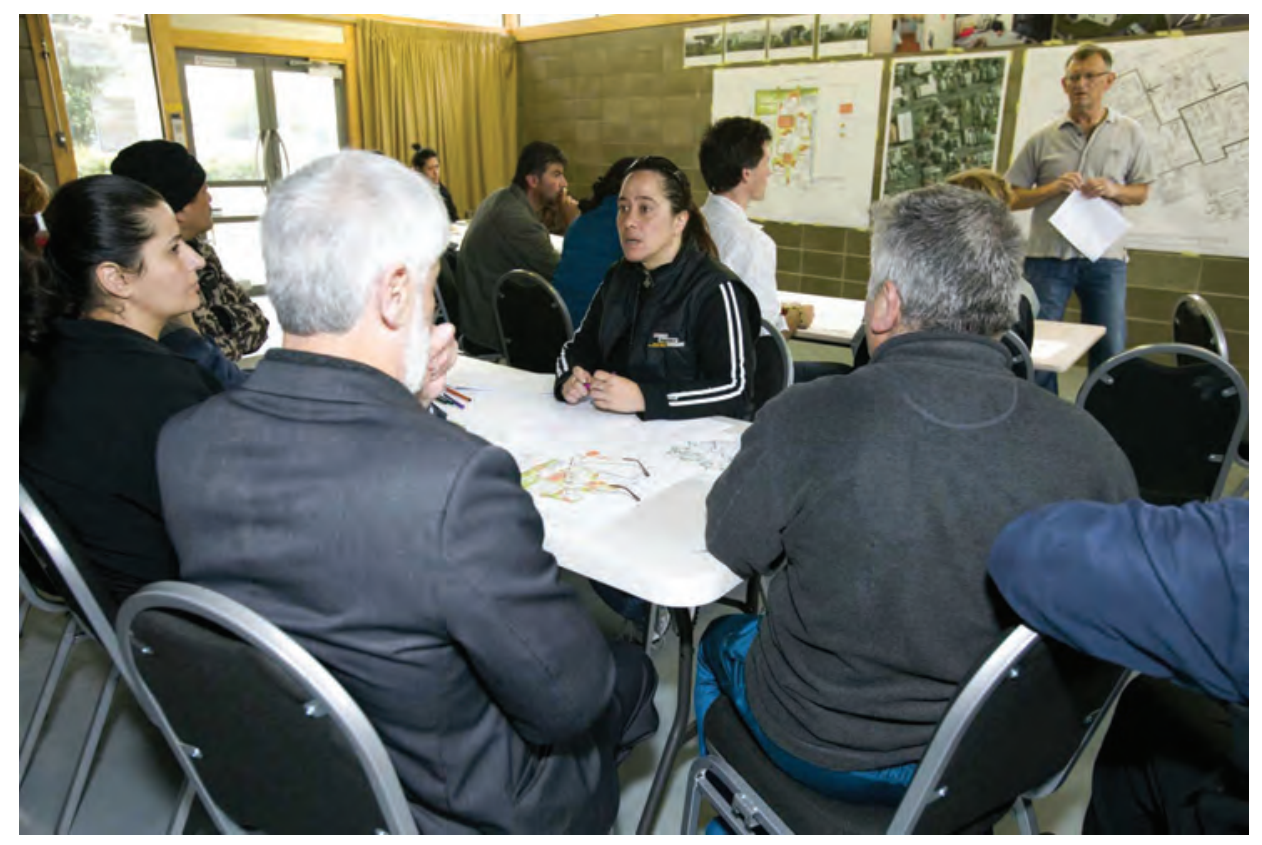




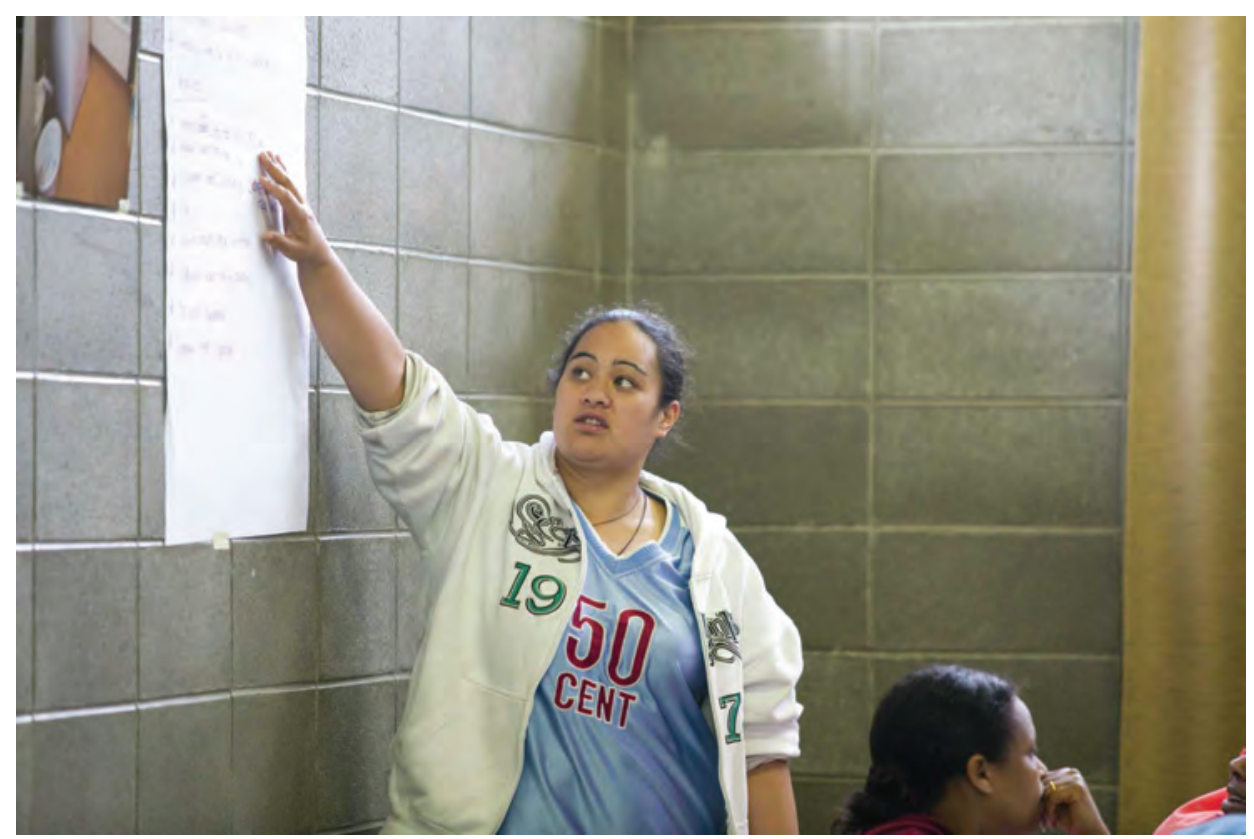

Figure 3.8 - Activate 1 (Nov 2008). Groups reported back findings to wider group

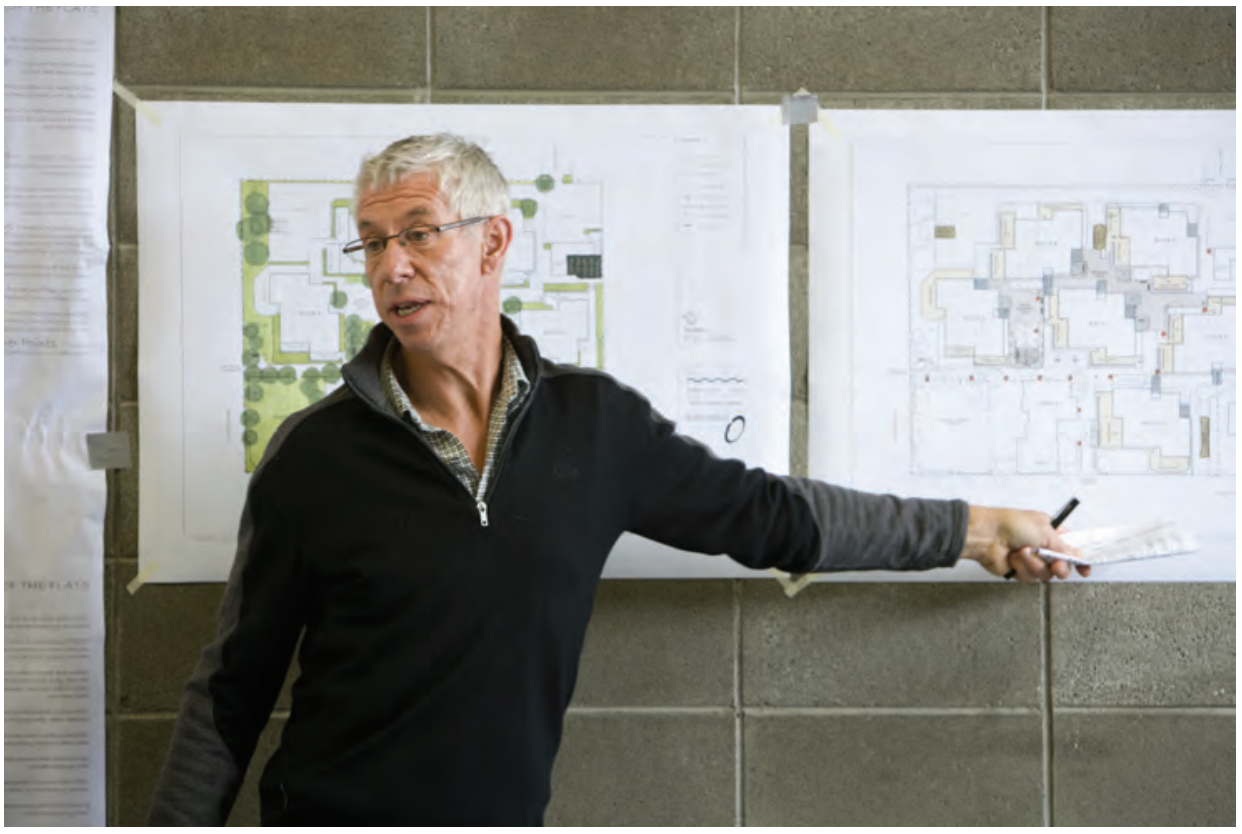

Figure 3.9 - Activate 2 (Feb 2009). Consultants presented a more developed design to tenants. 


\subsection{Kenya Workshop}

\subsection{Introduction}

This chapter presents the second participatory model that is the subject of the comparative analysis with WCC's approach - that by ASF at a participatory design workshop in Nairobi, Kenya. Section 4.4 discusses this participatory approach of the workshop in comparison to WCC's approach and in relation to the literature review.

Although the workshop's subject of slum upgrading in Kenya and my thesis' subject of housing upgrading in Wellington do seem to be rather disparate contexts, it proved to be an appropriate model to review. The literature review revealed that much of the leading edge best practice of participatory design/development, both historically and today, occurs in the developing world by NGOs and development agencies. This workshop was the third iteration of a participatory methodology developed by a team of researchers (Isis Nunez Ferrera, Naomi Shinkins, Alex Apsan Frediani and Matthew French), and had attracted leading participatory practitioner Nabeel Hamdi to attend and contribute his wealth of experience to the workshop. Furthermore, UN-HABITAT - an agency that has been seminal in the development of participation from the 1980's - was an official partner of the workshop. This reputable standing of the workshop, coupled with its educational objectives, made the setting of Nairobi, Kenya an appropriate place to learn avant-garde participatory tools and techniques and made the ASF workshop an appropriate model with which WCC's approach could be compared.

Attending the workshop made sense for logistical reasons too, as it gave me some practical experience in participation. Beyond simply reading, thinking, drawing (more typical exploratory tools of the architecture student), some action research was required to build my proficiency. 


\section{2 'Change by Design' - An Introduction}

The principal focus underpinning the workshop was an exploration of the opportunities and limitations of integrated community-led participatory design for responsive slum upgrading in Kenya, and the degree to which this community-led approach could not only build an improved physical environment but also recognise the social production of space; empower slum dwellers to be active agents of change; and build socially, economically, and environmentally sustainable communities. (French, 4)

The workshop was run under the banner of Architecture Sans Frontieres (UK) in partnership with Pamoja Trust (a Kenya NGO) and the Housing and Policy Section of UN -HABITAT. It took place in Mashimoni, a village within the wider slum area of Mathare Valley in Nairobi. Over 65 people were involved in the workshop on a daily basis over its two-week duration. Of those 65 , I was one of 22 international participants, some of whom had years of experience in participatory processes and others, like me, had little. Most were from the UK and Europe and both student and professional spheres were represented from a diverse range of sectors like architecture, sociology, economics and engineering. Another key group within the team was the group of 25 local students who were connected to the local NGO we worked with, and most of whom studied planning or a related discipline at the University of Nairobi.

I was a contributing author to a complete report of the workshop that discusses the workshop's process and findings in more detail than I will go into here. The remainder of this chapter draws from those parts of the report are most relevant to this thesis, and pays more attention to the processes we employed than the actual findings. The chapter then closes with my personal reflections as it transitions into the following chapter on my local case study. 


\subsection{Participatory tools and techniques}

I will briefly introduce the overall structure of the workshop as it assists in understanding the participatory tools and techniques I will discuss. The workshop methodology sought to explore the slum upgrading challenge in an integrated way, working at three scales concurrently: the institutional, regulatory scale; the neighbourhood, community scale; and the household, dwelling scale. Workshop participants split into three streams to address the three scales, and I selected the dwelling stream to take part in. As each stream employed variant participatory tools and techniques, I focus my analysis on those employed by the dwelling stream. Two other main components of the workshop that occurred outside the phases detailed below were a day symposium and a 'city visit' around Nairobi to see various approaches to slum upgrading being undertaken by others. Firstly, the symposium was hosted by UN-HABITAT at their global headquarters in Nairobi and was intended to bring together local and global stakeholders and encourage dialogue. It "brought international participants 'up to speed' with the context, and acted as the 'theoretical' precursor to the subsequent field activities" (French 10). As contributors to the symposium represented diverse perspectives (from government slum upgrading programmes to participatory design experts to residents), it became a very insightful participatory exercise in its own right. The second main component outside of the phases detailed below was the city visit. We viewed slum upgrading projects ranging from a local NGO's incremental approach to upgrading a small village to the Kenyan government's more heavy-handed upgrading of the well-known Kibera slum.

\subsubsection{Diagnosis: walking and talking}

This was our first 'on-site' phase and lasted one day. The emphasis of 'walking and talking' was to simply become familiar with the settlement of Mashimoni, both in a spatially/physically and also a more social sense. We understood its spatial arrangement and physical conditions through drawing, mapping and photographs and understood the social aspects through casual discussion with residents which was a sort of semi-structured interview. This phase was the least structured of the workshop; true to findings from the literature review, it was important we remained open to make discoveries that might alter the course of the remainder of the programme (Chambers 2002, UNCHS) rather than going on site to find evidence of any preconceptions we might have had. 


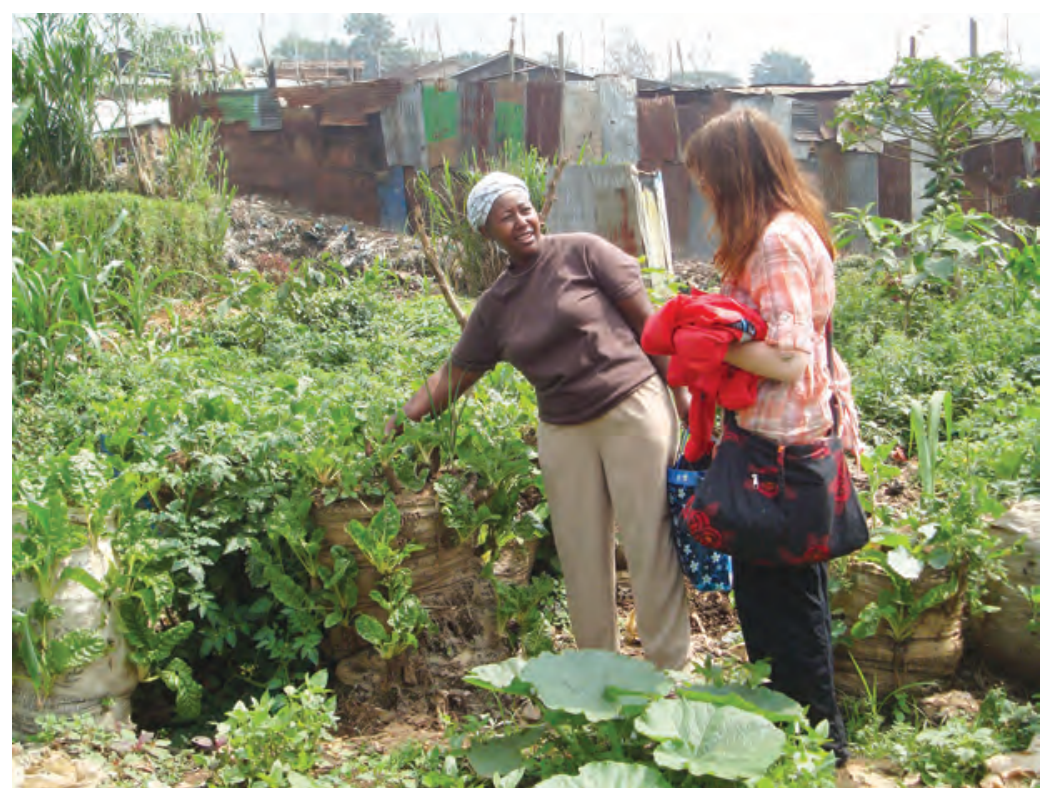

Figure 4.1 - 'Walking and Talking' in Mashimoni

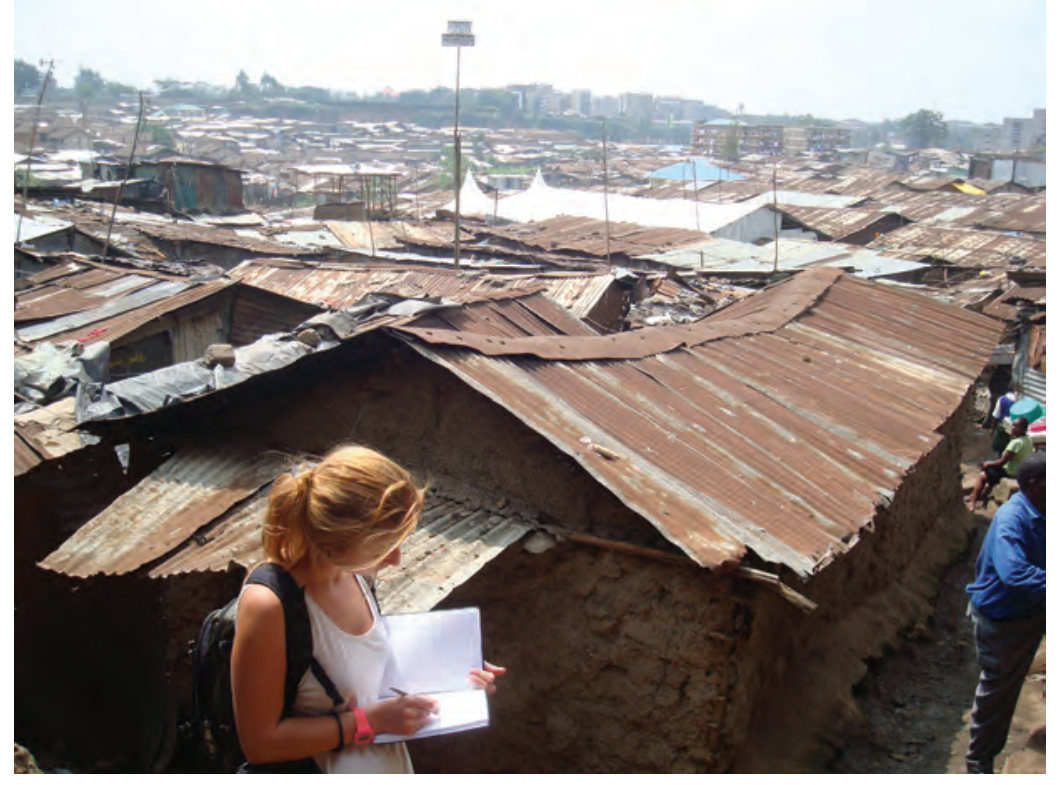

Figure 4.2 - 'Walking and Talking' in Mashimoni 


\subsubsection{Dreaming through drawing}

This next phase engaged the act of drawing as a means to reveal residents' values and aspirations around future housing. This technique was about 'drawing' in two senses; both the act of putting pencil to paper and, more broadly, pulling or extracting information and ideas from residents. Once information about a participant's household size and an overview of their housing history had been obtained, "one member of each household was asked to draw their dream house and describe the drawing, while the participants observed the drawing process to understand the hierarchy of importance of spaces they drew" (French, 106). It was the discussion around the drawing that proved the most informative, as participants revealed why - for instance - they wanted a verandah in the location specified, where fences were important, etc. In many cases, insightful discussion made up for a shortcoming in architectural drawing skills. "Imagining a life that was radically different to the existing physical conditions and ways of life" also proved difficult for some participants.

Figure 4.3 - 'Dreaming through drawing' exercises
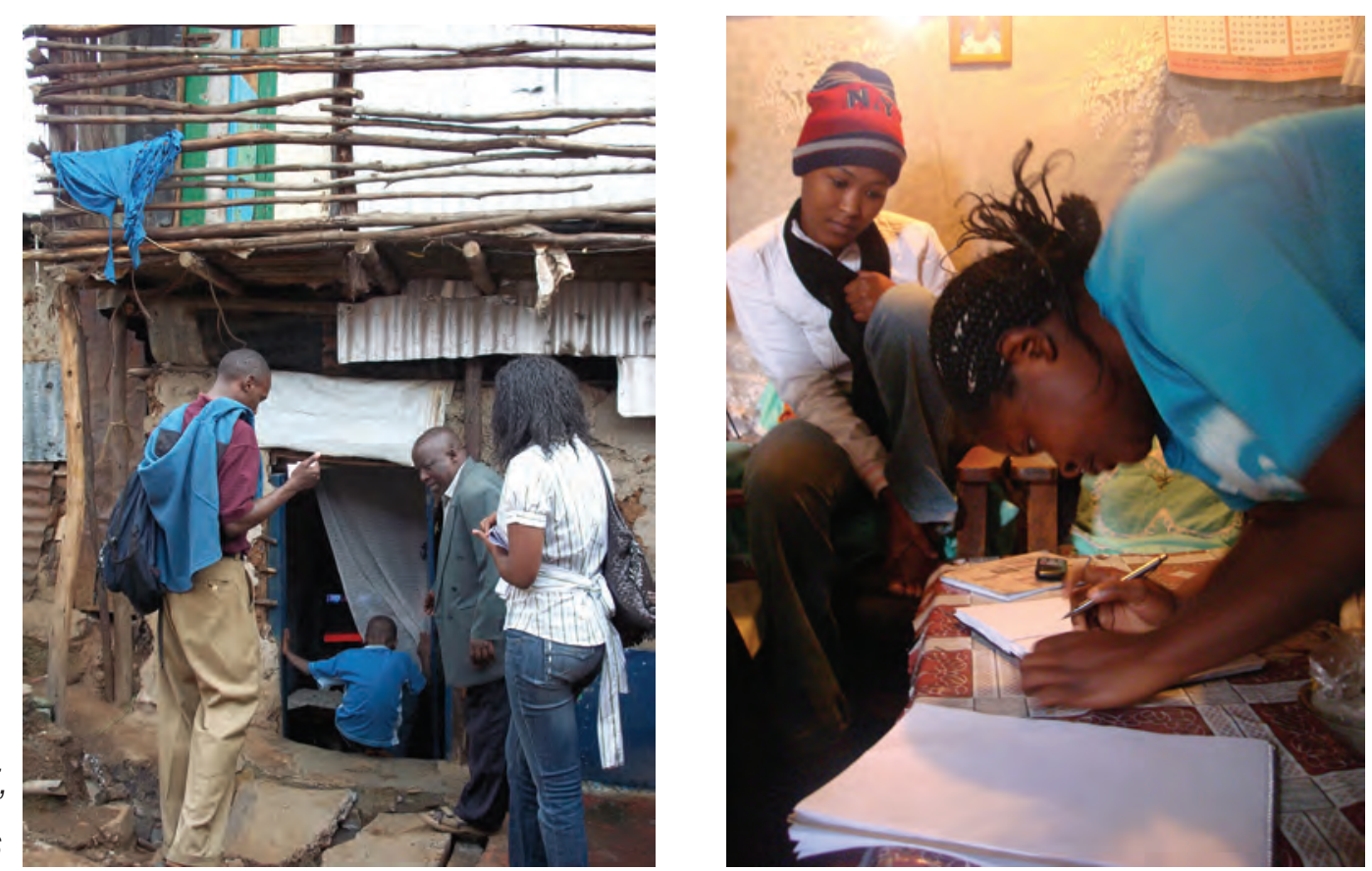


\subsubsection{Dreaming through modelling}

"In order to move on from the two dimensional drawings we prepared 'tool kits' which comprised cardboard models ('parts') of different rooms and dwellings spaces" (French 126). This kit of parts included multiple sizes of living rooms to bedrooms to toilets to 'unspecified' spaces that we hoped meant 'dream homes' weren't limited to only the parts we had thought to prepare. Also available for the modelling exercise were fences, trees, green spaces and verandas. Being in three dimensions, participants generally found this method very engaging, and some rich results were obtained (e.g. private open space was almost always in a rear yard for privacy, toilets were often separated from the main dwelling expressing values around sanitation, and security was a major concern). Solutions were surprisingly creative, with some two-levelled dwellings and even a well-considered courtyard house design.

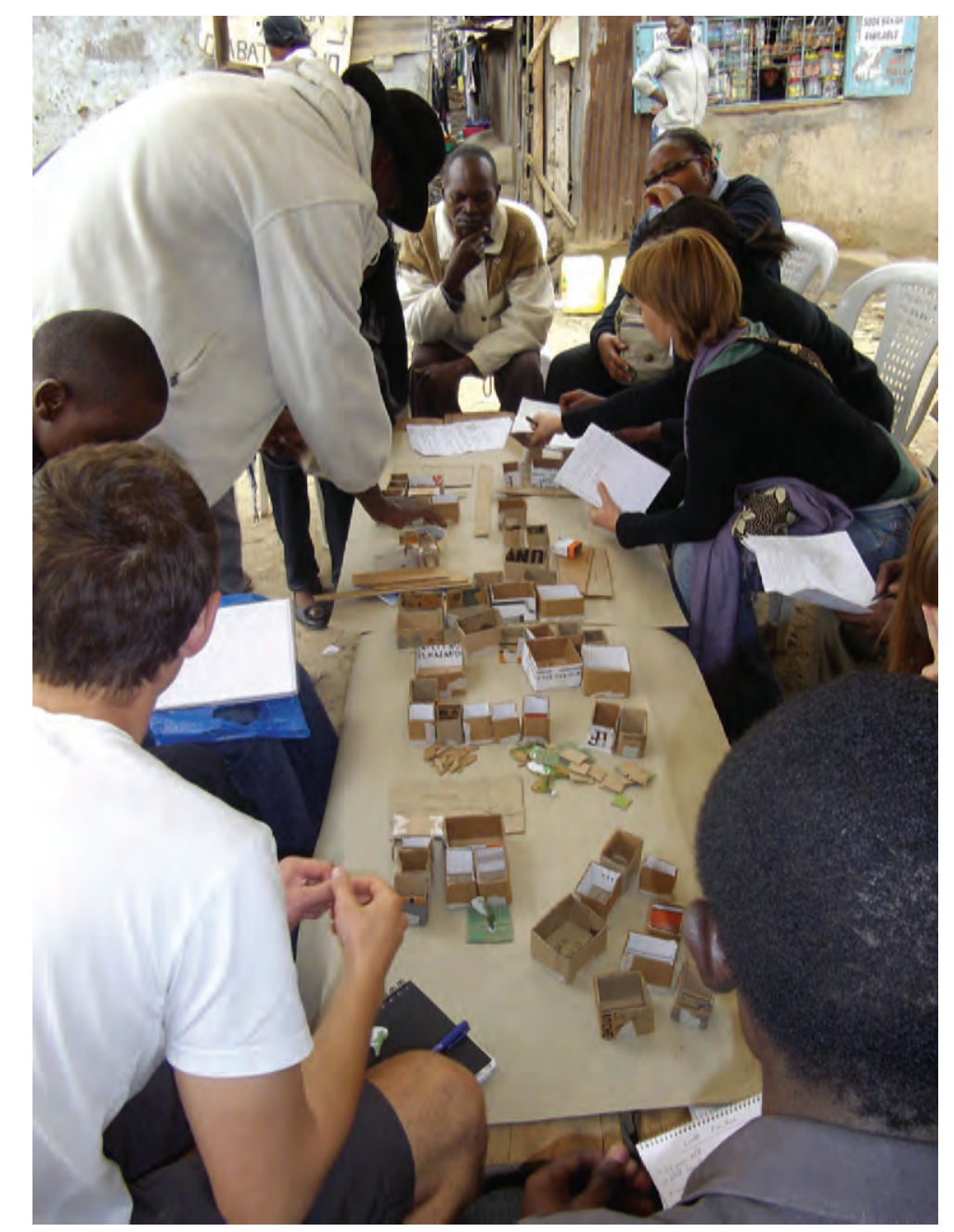

Figure 4.4 - 'Dreaming through modelling' exercises 


\section{BOX 1: The journey and dreams of Jacqueline}

One example of the 'dreaming through modelling' exercise at the Kenya workshop.

Written by author and extracted from 'Change by Design' report (2011:130)
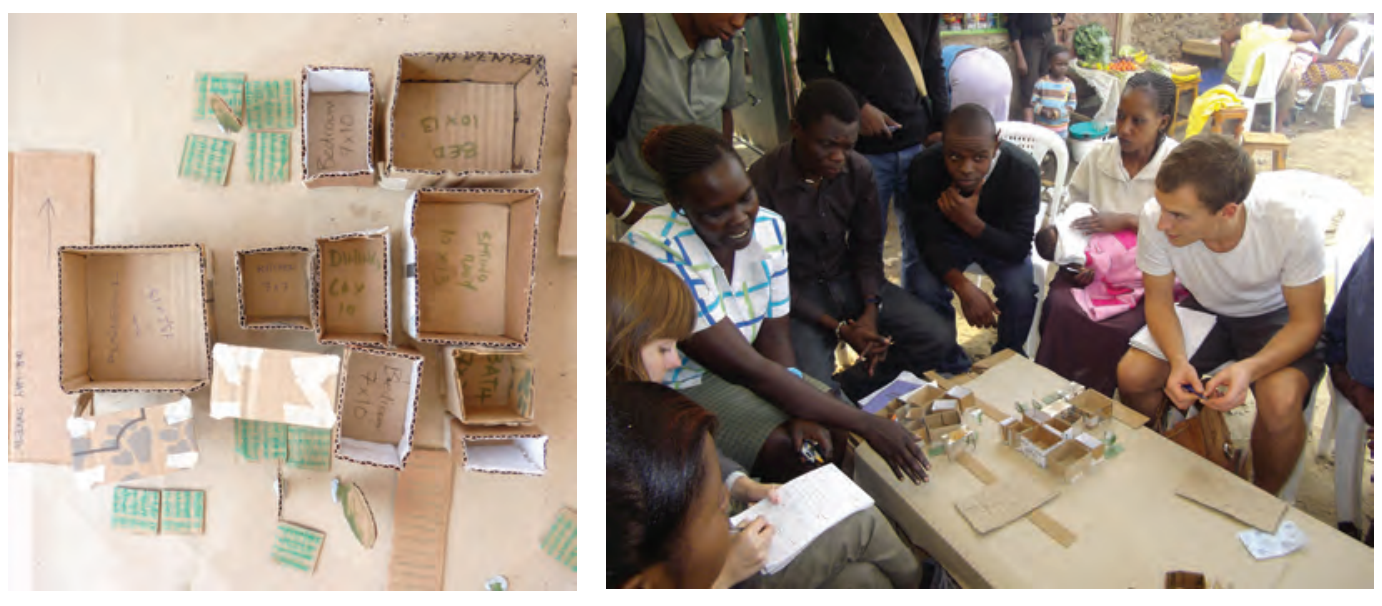

Jacqueline lives in a $10 \mathrm{ft} \times 10 \mathrm{ft}$ shack with her baby Juliet and two older sons, both around 8 years old. She operates a shop selling small (mainly imperishable) goods out of her home. Her rented shack has a small grilled opening onto the busy 'main street' of Mashimoni, through which her business operates.

Jacqueline modelled a modest dream house to support a very similar life to the one she currently leads. She dreamed of a dedicated room for her shop on the street edge with her 3 bedroom home behind. A veranda was situated outside the shop, and beyond that she wanted a playground developed for children. This was a sharp business move - as well as giving children something to do, which was a big concern of hers, it would attract parents to her property who would purchase goods from her! Not only that, but Jacqueline wanted to charge youngsters for using her playground.

This colourful example was one expression of the overall modest changes residents want to make to their environments. If land were more readily available, a dream of a small playground is reasonably easily attained. Indeed, a shortage of land seems to be the most stubborn obstacle in the way of residents' dreams. This lead to our suggestion of some more dense housing typologies to re-house the same number of residents with much more common outdoor space left over, for amenities like playgrounds. 
This exercise occurred in groups of four or so, with each participant working individually with a facilitator. The sessions got particularly interesting when we asked participants to combine their 'dream homes' together into a small neighbourhood, which included them having to collectively design street arrangements (major twoway roads, one way streets and pedestrian paths). This revealed differing values around how neighbours wanted to relate to one another and, most interestingly, invoked a more communal dynamic as participants discussed street options with each other.

In all, the sessions revealed great diversity amongst participants ideas for 'dream homes.' This exercise was well designed to cultivate that diversity. Box 1 is one example of a participant I worked with, and is extracted directly from the Change by Design report.

\subsubsection{Dreaming through typologies}

We then began the more challenging task of developing findings so far towards something of a coherent solution we could then combine with work the other two streams - institutional and community - were producing. This led us, the ASF participants, to develop four housing typologies with varying characteristics and densities based loosely on our findings from preceding phases of work. These typologies, presented through models, were: the tower; the detached house; the row house; and the gallery house.

These sessions had "the aim of moving to a deeper discussion of the relationship between housing development and communal wants and needs. The focus groups proved an excellent approach to undertaking negotiations between residents and highlighting the diversity of residents' needs, wants and aspirations" (French 134). It was at this stage of the workshop where more realistic trade-offs began, and discussion heated up accordingly. My biggest reflection on this stage of the work is how ready participants were to think about the good of the collective, exemplified by the common agreement that although 'the detached house' was the typology most resembling people's dream homes from earlier stages, it was least popular as participants could see that its density was too low to rehouse all of Mashimoni's 3500 residents (we had prepared the density calculations of each typology). 
Figure 4.5 - 'Dreaming through typologies' exercise

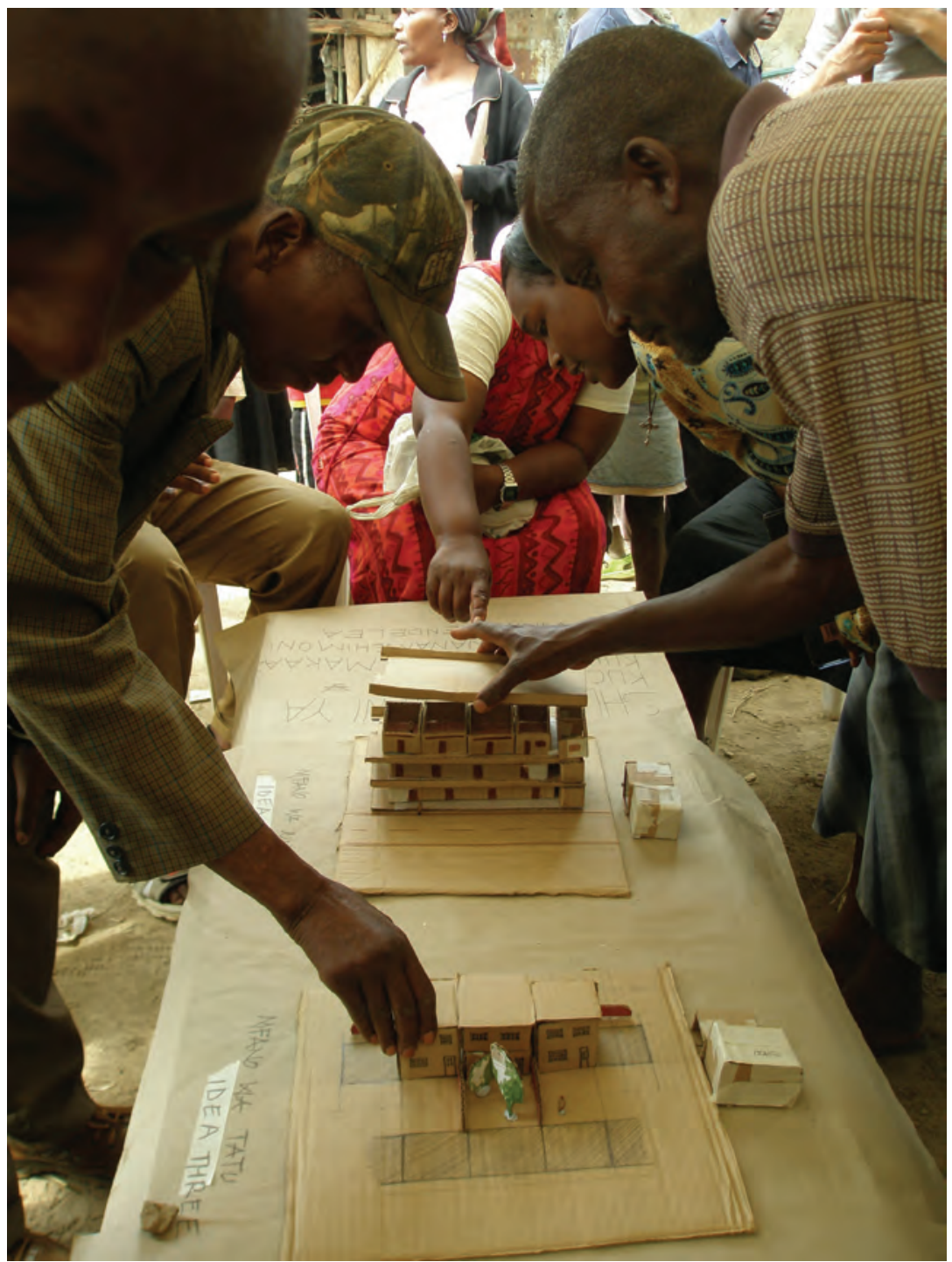

\subsubsection{Portfolio of options}

The workshop culminated in the 'portfolio of options' exercise. This brought the array of issues needing to be addressed through the slum upgrading process, that had been worked on in the three separate steams, into one arena.

Taking the form of a 'game,' the exercise engaged Mashimoni residents on site in planning their ideal upgraded settlement with a kit of parts on a scaled site model. Design games are a common participatory method, much like a jigsaw puzzle. "They are a highly visual way of allowing people to explore physical design options for a site or internal space" (Wates 2000:48).

One by one, individual elements from the kit of parts were introduced and discussed. Parts of the kit ranged from building procurement methods (e.g. self- 
build vs private contractor) to community facilities to be provided (e.g. halls, clinics, schools) to financing options (e.g. government grants vs residents' savings schemes). See Figure 4.6 for a full list of the kit of parts. Participants debated the benefits and disadvantages of each option for that 'part' and through this dialogue, having reached some degree of consensus, participants then placed chosen options down onto the site model (French 146).

The portfolio of options exercise was very effective in raising awareness of the complexity of the slum upgrading challenge, both for us workshop participants and the residents themselves. "International participants and residents alike gained a much more comprehensive understanding of not only the breadth of issues but also their interconnected nature" (French 163). Figures 4.8 - 4.11 are photographs of this exercise in action.

The diagram below now represents this information into a simplified diagram.

EVENTS

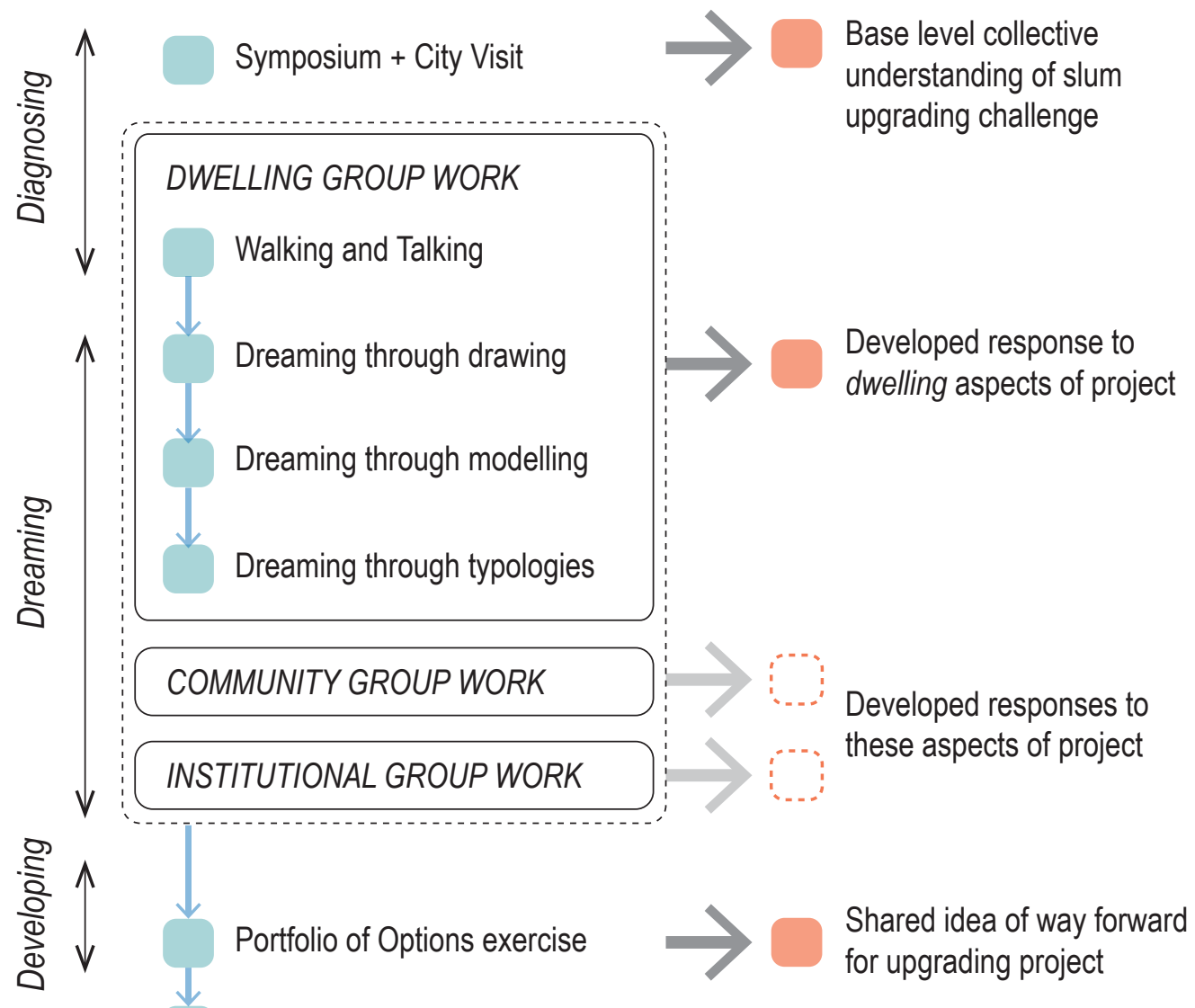

KEY OUTCOMES

Base level collective understanding of slum upgrading challenge

Developed response to

Exhibition

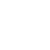

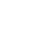



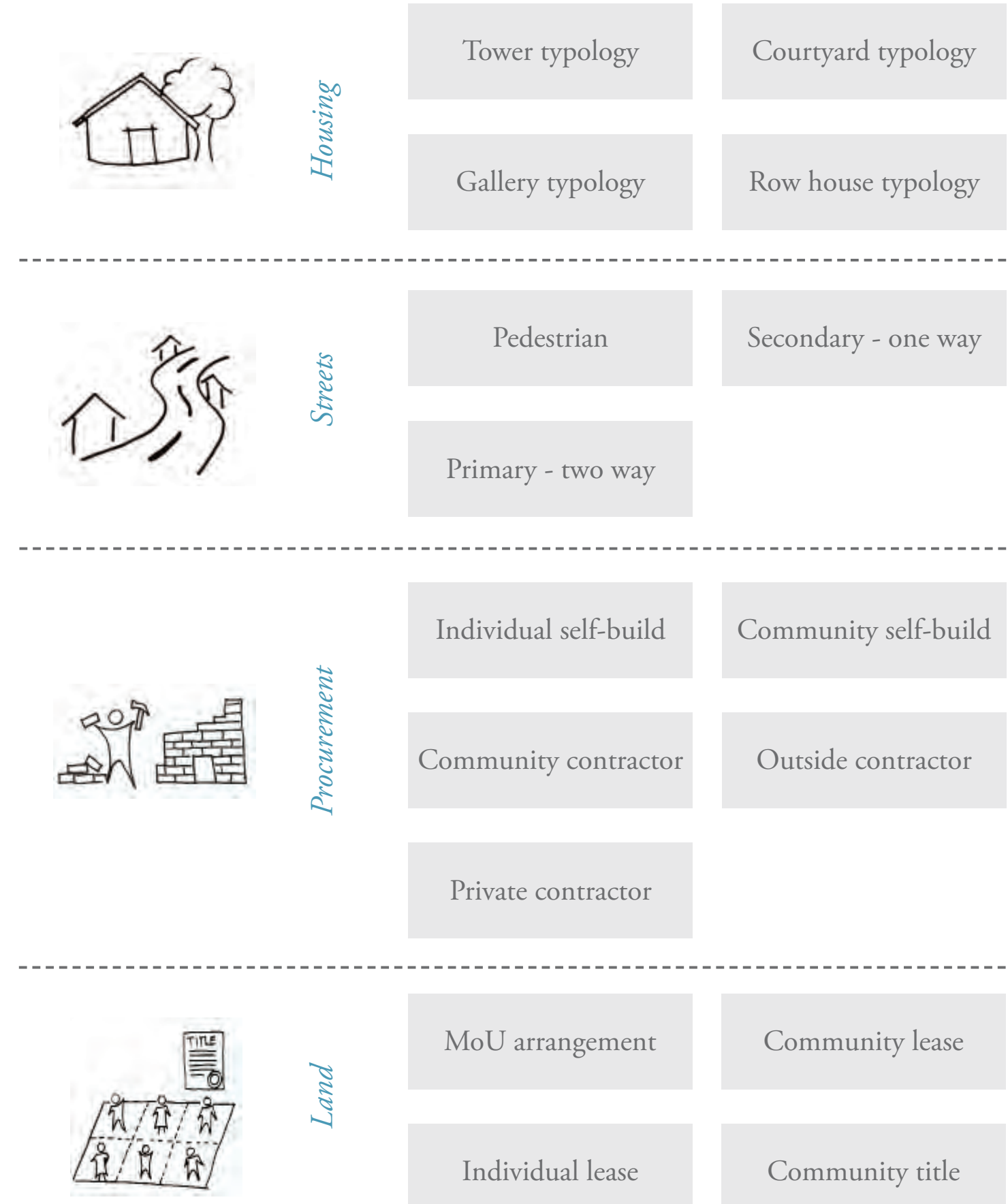

Individual self-build

Community self-build

\section{Individual self-build}

Community contractor

Outside contractor

Private contractor

MoU arrangement

Community lease

Individual lease

Figure 4.7 - Kit of parts'

for the portfolio of options

exercise [extracted from

Figure 162 of the Change

by Design report (French

2011:149)] 


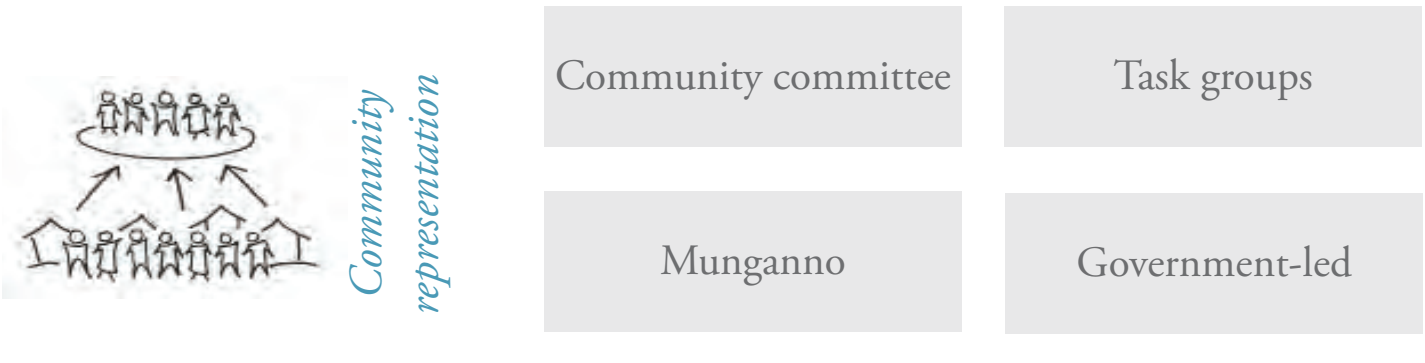

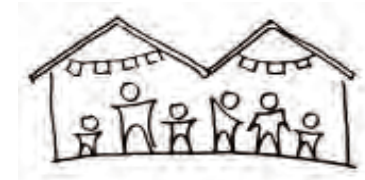

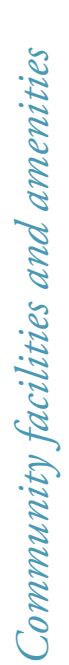

Community hall

Community hall

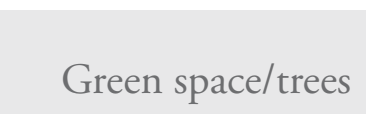

Health clinic

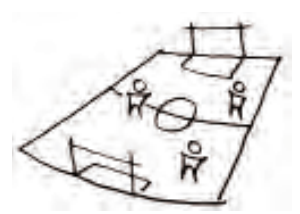

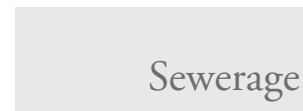

Sewerage

Market places

\section{Toilets}

Playground

School

Urban agriculture
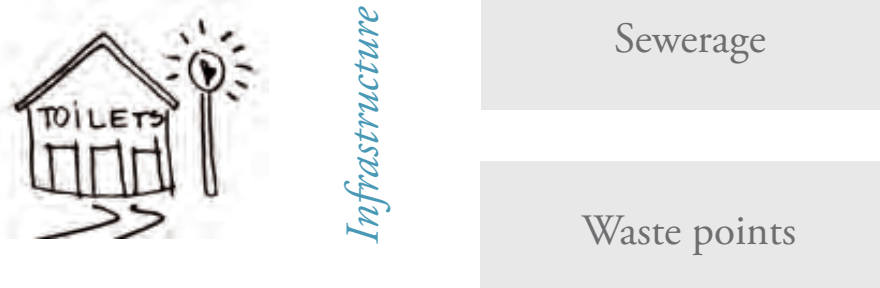

Water points
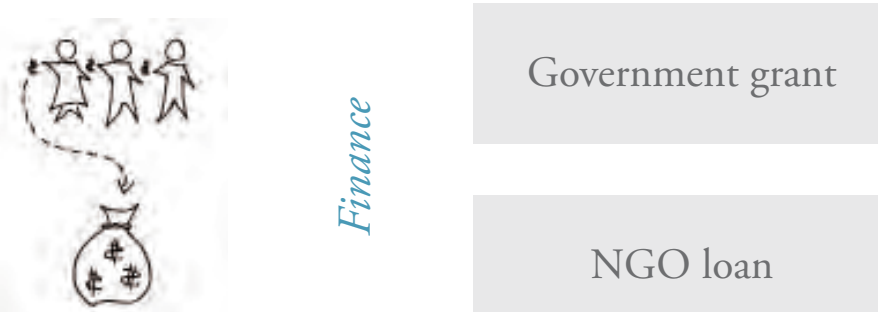

Bank loan

Lighting

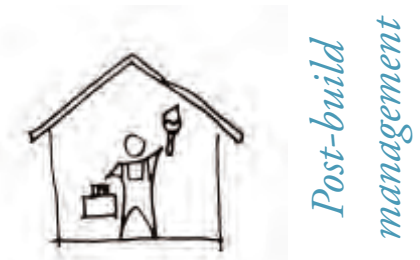

Private company

Community company
Government

Individual 
Figure 4.8 - Voting on a particularly contentious element of the portfolio of options exercise

Figure 4.9 - Facilitating a 'portfolio of options' exercise
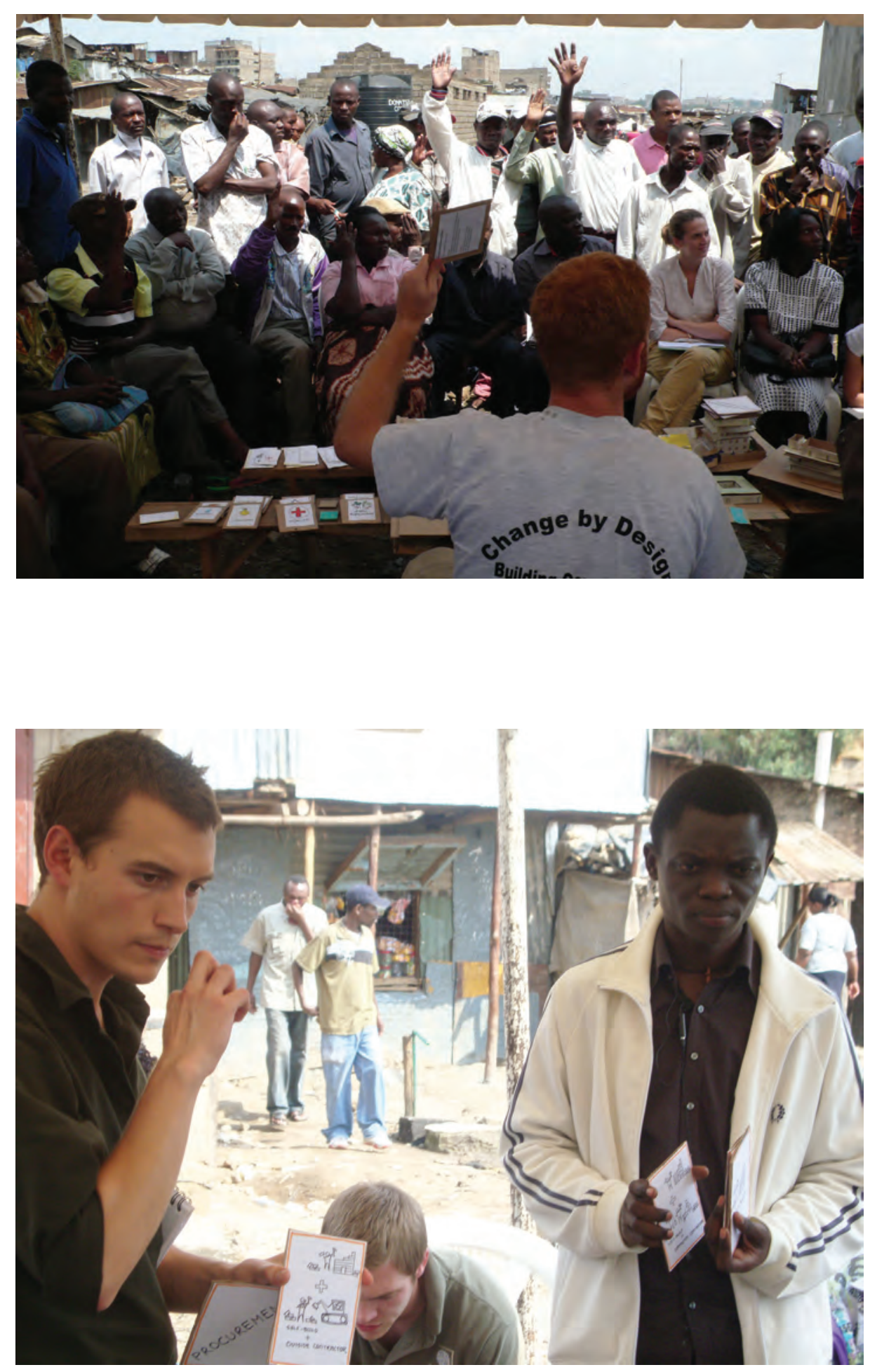


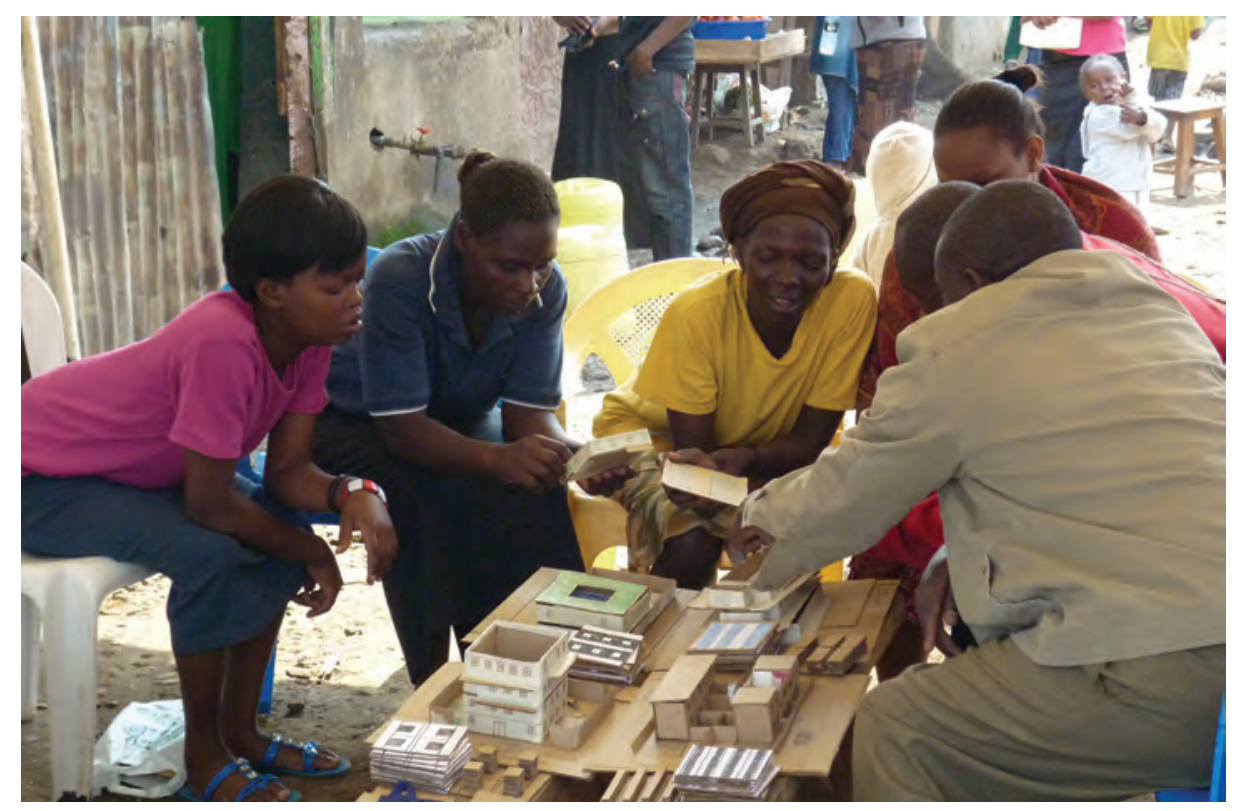

Figure 4.10 - Participants discussing housing typologies in the 'portfolio of options' exercise

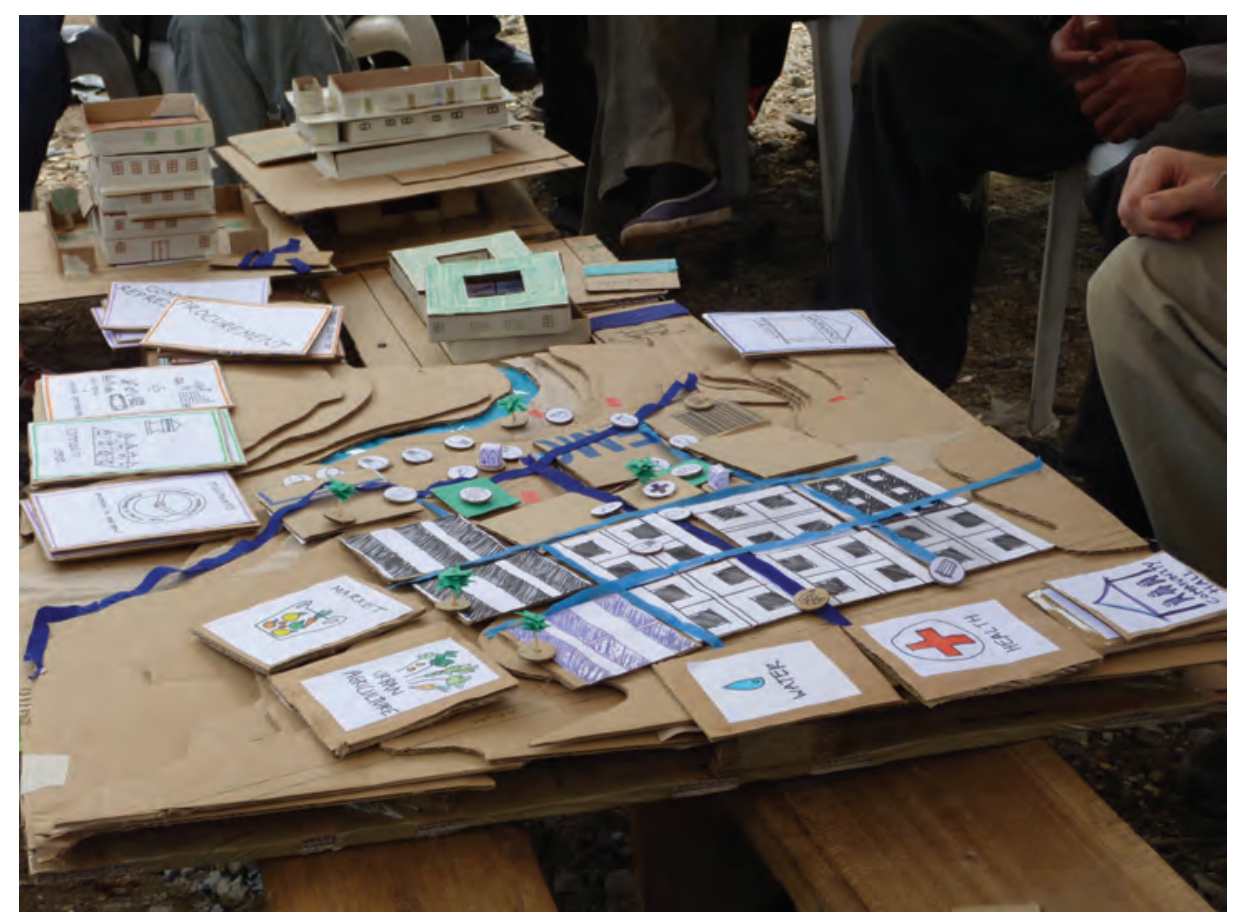

Figure 4.11 - One end result from a 'portfolio of options' exercise 


\subsection{Discussion}

This section reflects on some of the successes and challenges of the workshop, particularly in comparison with WCC's participatory approach.

\subsubsection{Work time consuming and had limited reach}

A challenge identified upon reflection of this process, particularly work done in the 'dwelling' stream, is the time consuming nature of the work due to people's attachment to the idea of 'home,' and their commitment to ensuring their points and ideas were sufficiently discussed. It seems difficult to accept that this challenge' of participatory work (that it is time consuming and labour intensive) is an acceptable excuse not to undertake participation in the first place, as is sometimes argued (Warburton 2005), however it must be acknowledged as a key challenge. That participants care enough to want to be intensively involved in a process simply reflects how important the outcomes of that process are to participants, and only confirms that there is sufficient 'demand' for the work to take place. That said, the challenge of how to undertake participatory work in cost and time efficient ways remains pertinent if the types of programmes we ran in Kenya are to scale up, and also if these more labour-intensive participatory techniques are to disseminate into developed world contexts like WCC's Housing Upgrade Programme. This challenge perhaps goes some way to explain a key thread that emerged from the literature review, that participation is too often 'under-done' - that rudimentary consultation is too often passed as participation, as it is easier to carry out. The 'Activate' sessions by WCC, where a small number of staff and professionals facilitated a large meeting of tenants is certainly simpler to carry out than the fortnight of full-time interviewing and workshopping we did in Nairobi.

Relating to this, another limitation of the process was the relatively small number of residents the team was able to reach. Even with 50 or so 'facilitators' working for a full two weeks, only a minority of Mashimoni residents (perhaps only 150 people of a total 3,500 residents) were actually engaged in the more intensive components of the process" (French 140). Further to this, it took effort to ensure we were continually engaging fresh faces in the work, as a small group of residents (approximately 10) would turn up to every session we ran, some even wanting to participate in the same exercise twice. We had to be careful not to limit capacity for other participants. 


\subsubsection{Managing stronger and weaker voices}

Another challenge encountered was how to manage stronger voices that overpower focus group sessions - a well known issue in participatory work.

In Mashimoni this limitation was overcome, to an extent, with effective facilitation. As dominant participants were always men (without exception), running a women-only focus group was useful to obtain valuable insights from women (French 140).

This remains a key challenge in any participatory work, as facilitators manage stronger voices and seek out weaker voices. There seems to be no difference between this challenge at both the ASF workshop and also throughout WCC's process. A response to this challenge that proved successful in Mashimoni was to carry out a diverse range of activities that meant as many voices were heard as possible. The more intimate one-on-one sessions in homes (like the dreaming through drawing sessions) provided an alternative channel to the group sessions that was more conducive for some people to share their opinions and ideas. That said, there is value of group exercise that should not be overlooked, for example, some of the most insightful discussion in workshops occurred between residents themselves, as we facilitators simply looked on. What is important is that a range of different activities are carried out, catering to and providing a forum for a range of personality types.

Interestingly, this learning from the ASF workshop further developed a finding from the literature review. I had learnt that participatory processes inescapably engage in power relations (see 2.3.5), but had considered that only between facilitators and participants. What became clear in Kenya, and what I would imagine occurring equally in Wellington, is that power dynamics within a group of participants can require just as much attention.

\subsubsection{Fighting a bad reputation}

Our constant presence over the two week period, and our genuine interest in something as close to residents as their settlement (especially their individual dwellings) seemed well-received by participants. Residents were apparently used to seeing 'outsiders' visit the settlement to undertake research, make promises of 
improvements and never be seen again. This most unfortunate reputation 'foreign researchers' had accumulated in a slum context did result in some sceptical residents not wanting to be involved in our work - direct evidence of much of the cynical feeling towards mal-practiced participatory work from the literature (Cooke 2001, 2004). Whilst this was disheartening, and has indeed caused necessary critical reflection around whether or not we were 'any better than those other researchers,' it was heartening to receive generally positive feedback from residents at the end of our workshop. Taking time to provide a listening ear for residents and returning daily for two weeks to develop ideas in a participatory manner, it seems, made a great difference in helping residents feel respected and valued (French 141). This seems to align well with Sanoff's point that good participatory process should break down traditional barriers between 'expert researcher' and 'researched community' (2007:59). Mashimoni residents seemed pleasantly surprised with our level of genuine engagement and seemed to respond well to being engaged as 'full participants' not 'subjects' of an outsider's research project. Interestingly, as in 4.4.2, a very similar finding applies to WCC's process. Through interviewing tenants at Te Ara Hou, it became clear that the reputation of Council in tenants' minds had a great impact on how they felt about the

Figure 4.12 - 'Tiles' of housing types (row, tower, etc) allowed for quick calculations of how many people a proposal would house.

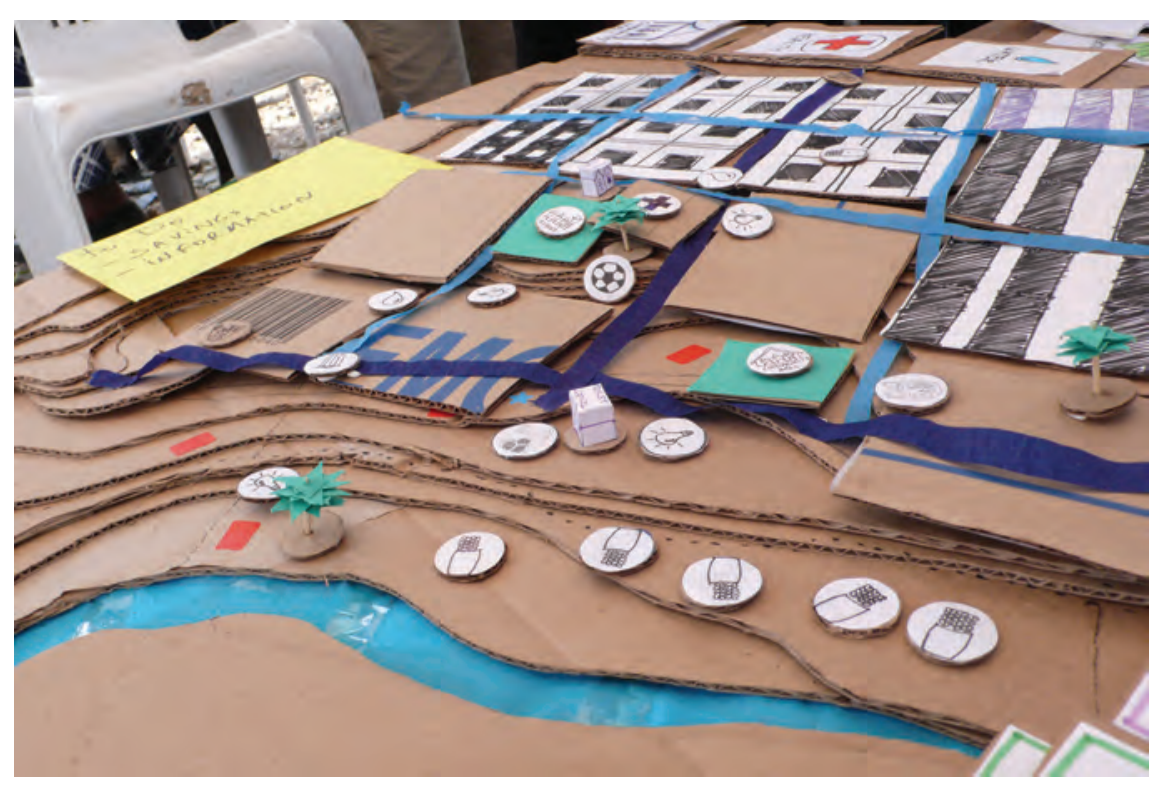

upgrade process. Some felt close to their landlord and were thrilled with results, while others haboured ingrained resentment and cynicism towards Council and the upgrade, stemming from years of what they saw as poor treatment. Whether in Kenya or Wellington, it is clear that the reputation of facilitators (or those who have gone before them) impacts greatly on how participants respond. 


\subsubsection{Building participants' capacity}

Another positive reflection was "the degree to which residents' capacity was developed, to understand space, the use of space, and how to represent space through in-depth, prolonged, one-on-one contact with residents" (French 141). This was most clearly demonstrated in the final 'portfolio of options' exercise, where the various densities of different housing typologies were being discussed over the scale model. The evening prior, we had worked out how many households each typology housed across a common 'tile' size (to scale), so when it came time to discuss how much area of each housing type should be proposed we could calculate the number of households an option would house on the spot.

We were all understanding housing densities and land area in an irrefutable, spatial and scaled way. Discussing density in such a way trumped the impassioned - yet unfounded - opinions from all of us about which typology we personally thought was too dense or not dense enough, which could have consumed our time and energy unproductively. As the report points out, "the skills acquired by Mashimoni residents will be useful when residents and their representative organisations are reading, refining, and negotiating with future (professionally produced) upgrading plans" (French 141).

The enduring effects of a participatory process that go well beyond a particular physical project (like building participants' capacity to understand a scale model) is something that has become clear though reviewing both the ASF workshop and WCC's process. In Wellington, what emerged as an enduring effect of the process was a strong sense of attachment to place by having been involved in its creation (particularly evident with the playground project). I would now add that an important characteristic of participation is that its effects endure beyond the implementation of a physical project my 'definition of participation' (2.3).

\subsubsection{Drawing and modelling as tools}

The drawing and modelling exercises proved to be very fruitful for different reasons. Both tools were effective bridges over the language barrier encountered between international participants and locals. That said, local students translating discussion (or some residents' proficiency in English) was critically important too, as the discussions over the drawings and models were integral to the exercises. 
"The order in which residents drew dwelling elements gave an indication to their priorities" (French:138), which we found to be insightful. While some were confident drawing, others were not and for them, particularly, the modelling exercise was useful. We generally found quick spatial models to be a highly engaging method that most, if not all participant, could understand. WCC's process did not engage participants in either drawing or modelling. The Activate sessions moved too quickly for participants to have time to draw. Even if there was time, I suspect drawing would be less useful in an upgrading/renovating project, as the subject for a drawing would be far more complex than the simple 'dream house' exercises carried out in Kenya. Drawing and modelling at the ASF workshop certainly reinforced the finding from the literature review that participation highlights diversity in people's preferences and dreams (2.3.6). Drawings (and photographs of models) were a useful physical record of the wide range of ideas and preferences that were expressed.

\subsubsection{Considering the collective}

Overall, residents were modest and realistic in their dreaming. In fact, if anything, they were too modest in the initial dreaming exercises and we had to encourage them to dream bigger (as it was through 'dreaming big' that a clearer picture of one's values and aspirations could be established). In later stages, as more constraints came into focus, residents were very prepared to compromise and consider their neighbours for the wider collective good. "[their housing choices reflected a] modest view to upgrading Mashimoni, as well as their desire to have all existing residents be accommodated" (French 2011:139). This observation proves wrong a common antipathy to participation that it only unrealistically raises expectations, and there is little point in participants preparing 'shopping lists' that can't be delivered upon. In Mashimoni, we experienced a much richer process than that; one in which participants understood collective limitations and were keen to work realistically within those limitations. This finding directly reinforced the point from the literature review that a good process can 'bring into being a collective intelligence' (2.3.7). 


\subsubsection{Final comparisons between WCC and ASF}

Moving forward into the remainder of the thesis project, two important differences between the ASF Kenya workshop and WCC's Housing Upgrade Programme must be pointed out. Firstly, the educational element of the Kenya workshop. As well as intending to have some positive impact on slum upgrading projects, its organising body Architecture sans Frontiers (ASF) plan these workshops to provide a learning environment for students and practitioners keen to explore participatory methods of working. This is important to note as WCC's Housing Upgrade Programme sits in quite a different reality of pressing upgrading work grounded very much in practice. This contrast also highlights the second point that in Kenya our work was not to directly inform an upgrading project (although it is intended one will occur sometime in the near future, and our work will impact that) and could therefore 'dream bigger' with less limitations. Again, WCC's programme is much more limited in that sense.

This workshop gave me the practical experience in participation that I needed to compliment my review of literature. It provided practical reinforcement of certain findings from the literature review, and certainly revealed much more complexity and fun than I had anticipated. As has been discussed, comparisons with WCC's participatory process were very easily made. Focus now returns to Wellington as I get closer to proposing a developed participatory approach by conducting 'a design experiment'. 


\subsection{A Design Experiment}

This chapter builds upon the comparative analysis of two processes (discussed in chapters three and four) and works towards a developed participatory approach by conducting 'a design experiment' at Te Ara Hou Apartments in Newtown. Firstly, some detail is provided on the site Te Ara Hou (5.1), then my own tests of participatory exercises at Te Ara Hou are documented (5.2). Section 5.3 establishes a brief and builds understanding around the two components of that brief through case study research on WCC community rooms and medium density housing precedents. Finally, 5.4 presents a proposed physical intervention for the site, informed by all preceding sub-chapters. This chapter closes with discussion on all stages of this 'design experiment', particularly how the more 'designer-centric' stage of 5.4 slots into a wider participatory process. 


\subsection{Case site}

\subsubsection{Arlington Apartments - A False Start}

The case site Te Ara Hou was not my initial preferred site. I had initially selected the site of Arlington Apartments in Mt Cook for three primary reasons. Firstly, it is a site in WCC's portfolio that was yet to be upgraded and was coming up in the programme. I was optimistic that my thesis work could have some positive effect on the real upgrade of the complex. Secondly, it is a large site which seemed to contain sufficient complexity in its form and surrounding urban context to sustain a year-long project. Its housing typologies are diverse (from a tower block of apartments to lower rise cluster housing) and the complex spans several blocks, introducing interesting urban-integration challenges. Further to this, throughout the site are several opportunities for infill development which appealed as it left open a wide range of options for architectural intervention. I was looking for intervention opportunities which I knew I needed to explore later in the project. The third reason why Arlington appealed as my case site was that I had developed relationships with some residents and neighbours to the complex who gave me important connections with the tenants I needed to engage through the project. Arlington Apartments seemed to be the prefect case site for my study.

My completed work at Arlington Apartments included archival research of the complex's conception and history, discussions with the Arlington's original designer (lan Athfield) and WCC staff about the current state and future challenges of the complex and 5 semi-structured interviews with residents. I had begun designing a participatory approach that could explore opportunities for infill housing, as the site presented many opportunities for increased site coverage. Unfortunately for the project, as I approached formalising research agreement with WCC (required to undertake more formal interviews and workshops), they informed me that Arlington Apartments had became an unsuitable location for my research to take place. This was, in essence, due to Arlington being rather contentious complex in the Housing Upgrade Programme, and my thesis work was seen by WCC as potentially further confusing the upcoming upgrade of the complex. WCC suggested an alternative site for my work: Te Ara Hou Apartments in Newtown.

At WCC's instigation, I re-located my design case study to Te Ara Hou. This shift allowed me to retain a close working relationship with WCC, which was important for the project. Central to the over arching aim of my thesis was to examine how 
participatory design is carried out in the Housing Upgrade, so I needed their continued involvement and cooperation in the study. I could make a new site work.

What made the shift to Te Ara Hou difficult at first was that the complex lacked many of the attributes I thought were important for my case site to possess. To refer back to the three primary reasons why I had initially selected Arlington Apartments:

- Te Ara Hou had already been upgraded, so offered less prospects of my thesis work positively effecting a real upgrade;

- Te Ara Hou is a smaller site with less diversity in its housing types within it, less complexity in its urban conditions and, importantly, fewer opportunities for infill intervention as the site seemed already close to its site coverage capacity;

- Less significantly, I had no existing knowledge of the complex and no existing relationships with residents.

That said, I proceeded focussing on the positive aspects of this change; studying an already-upgraded complex provides an opportunity to assess the success of the upgrade (a post-occupancy evaluation, of sorts) and less infill development opportunities didn't eliminate all opportunities for architectural exploration at the site. I decided to begin participatory design activities and see what that process revealed.

\subsubsection{An introduction to Te Ara Hou Apartments}

Te Ara Hou Apartments is a unique complex of housing, as expressed in the startling headline by The Evening Post (see Figure 5.1). The complex was designed by Kofoed, Arnold and Partners and built in 1975. Like a lot of WCC's housing portfolio constructed in the 1960's and 1970's, it was as part of a bold council-led urban renewal (also referred to as 'slum clearance') programme proposed for the area. The full plans for the renewal of a 20 acre are south of Constable St were never carried out, although Te Ara Hou and another complex on Hiropi St are two lasting remnants of these bold 'Golden Age' plans (Evening Post Article).

Te Ara Hou's 67 units are divided amongst six four-storied clusters which cover a site of 0.8 hectares (two acres). Although it is a typology of housing quite different from the surrounding pattern of early 20th century stand-alone dwellings facing the street, it visually blends in with its surroundings reasonably well due to the blocks' quirky 'oversized house' forms. 


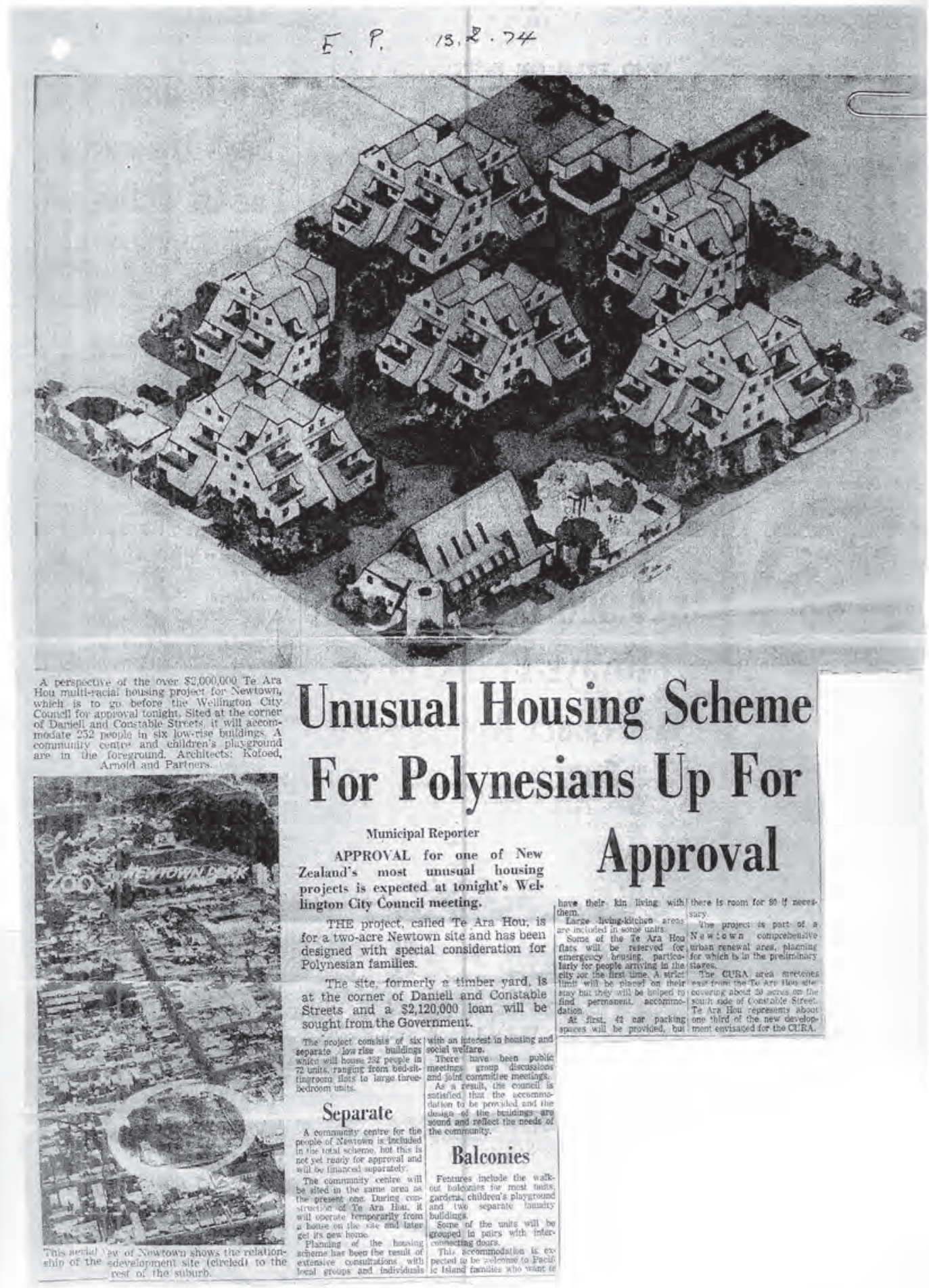

Figure 5.1 - Evening Post article dated 13.03.74. 
Figure 5.2 - Block en-

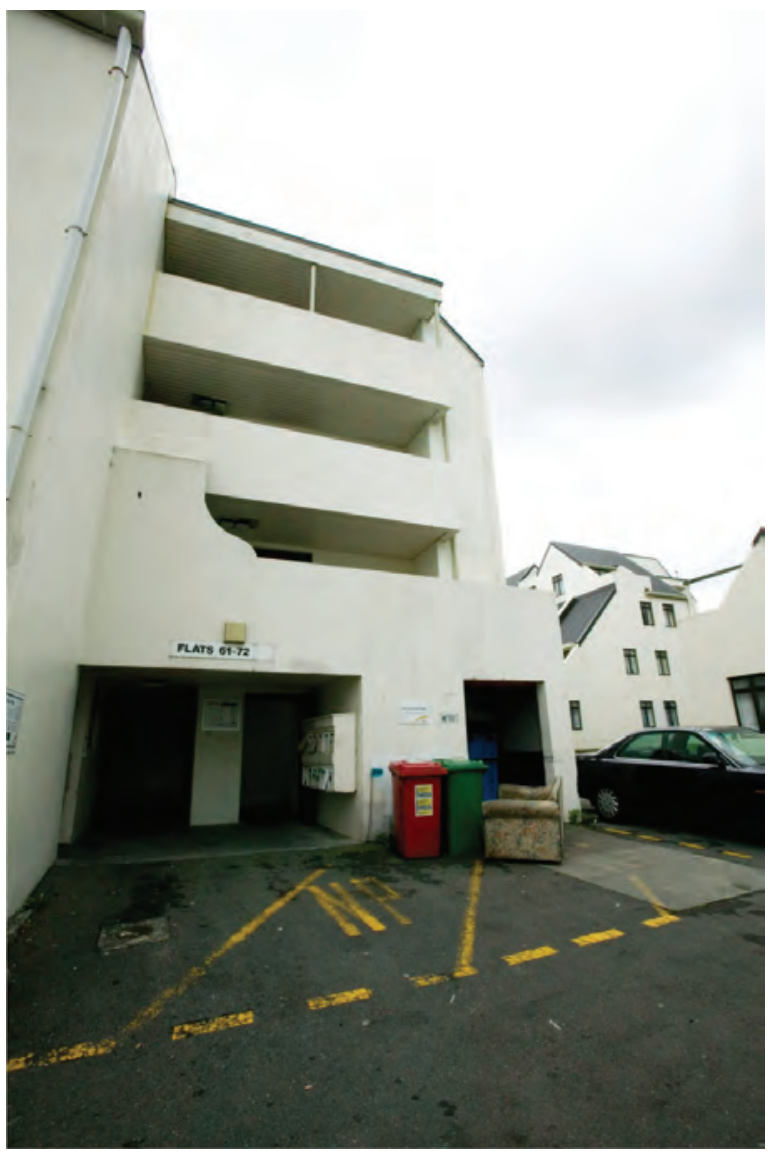

trance pre-upgrade

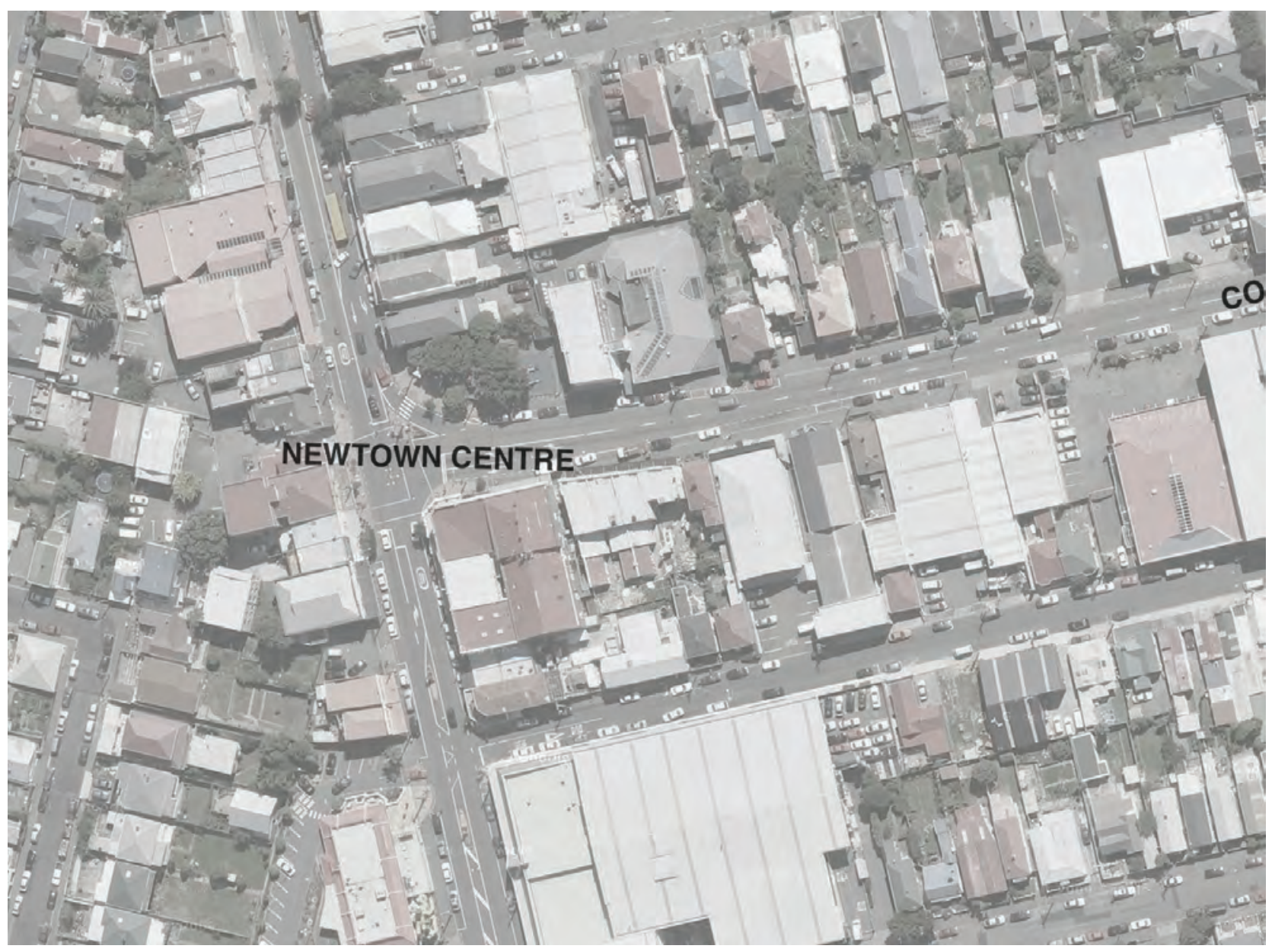




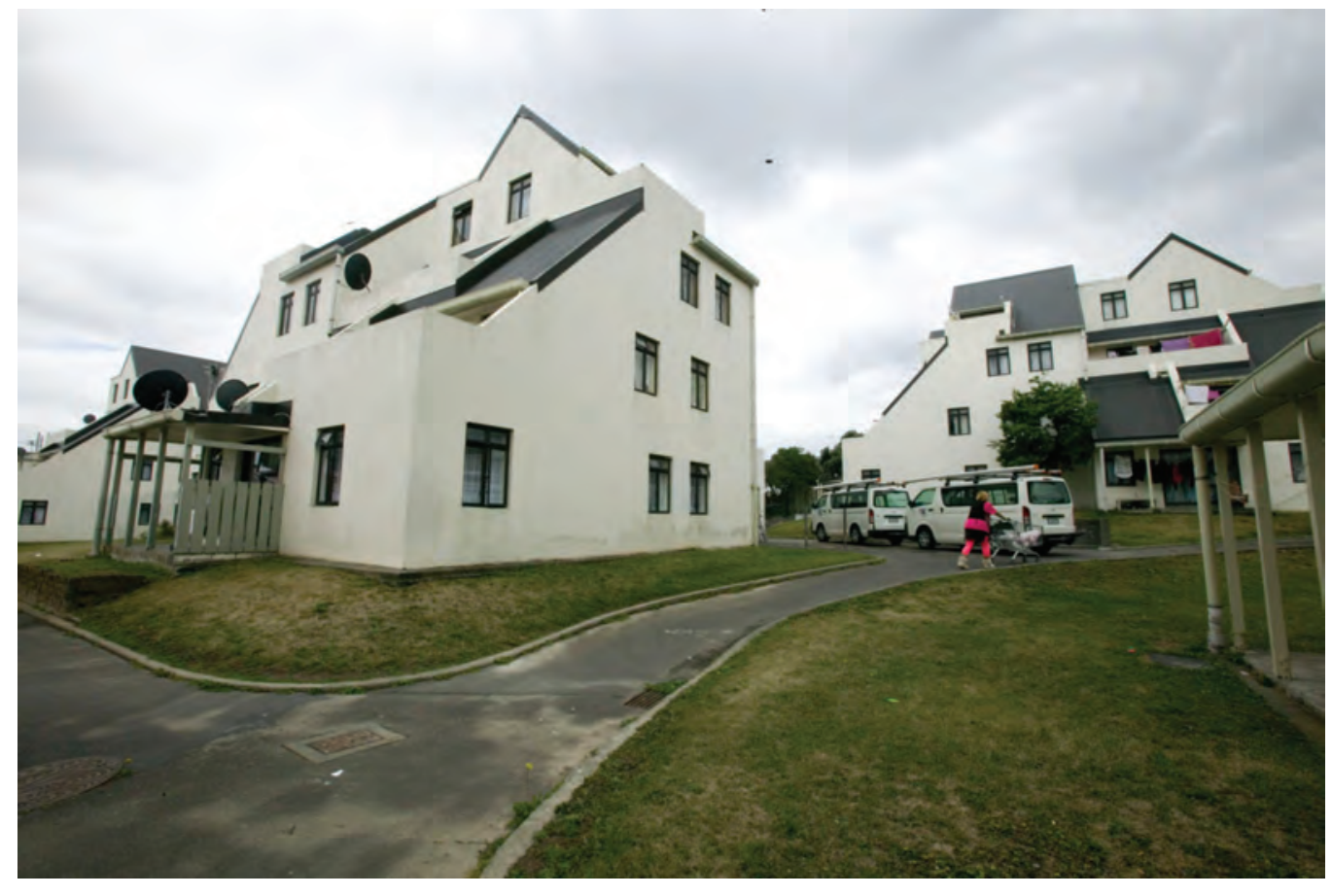

Figure 5.3 - Grim

outdoor space prior to upgrade

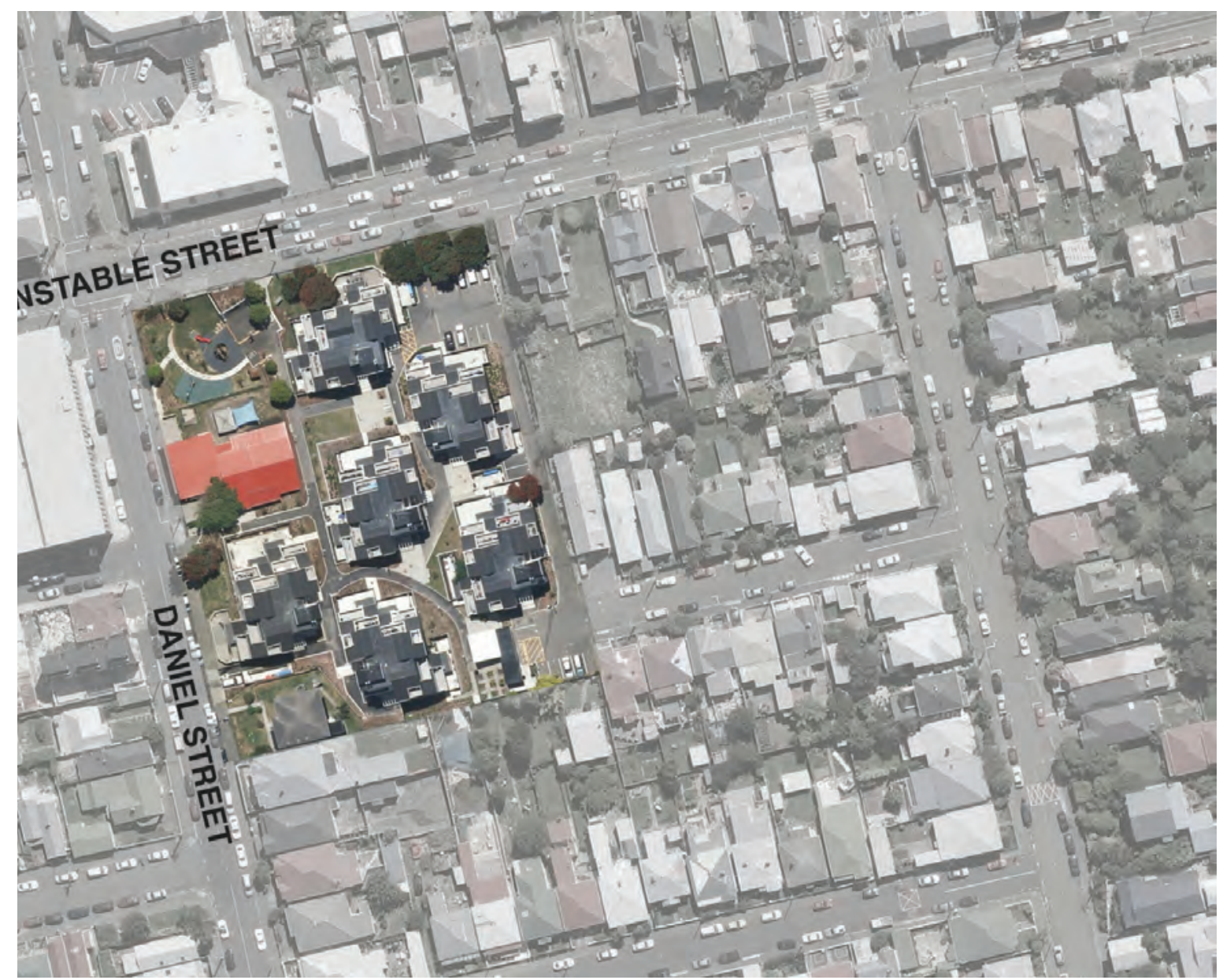

Figure 5.4 - Site Plan of Te Ara Hou (N.T.S) 
Figure 5.5 - Reworked outdoor space following upgrade

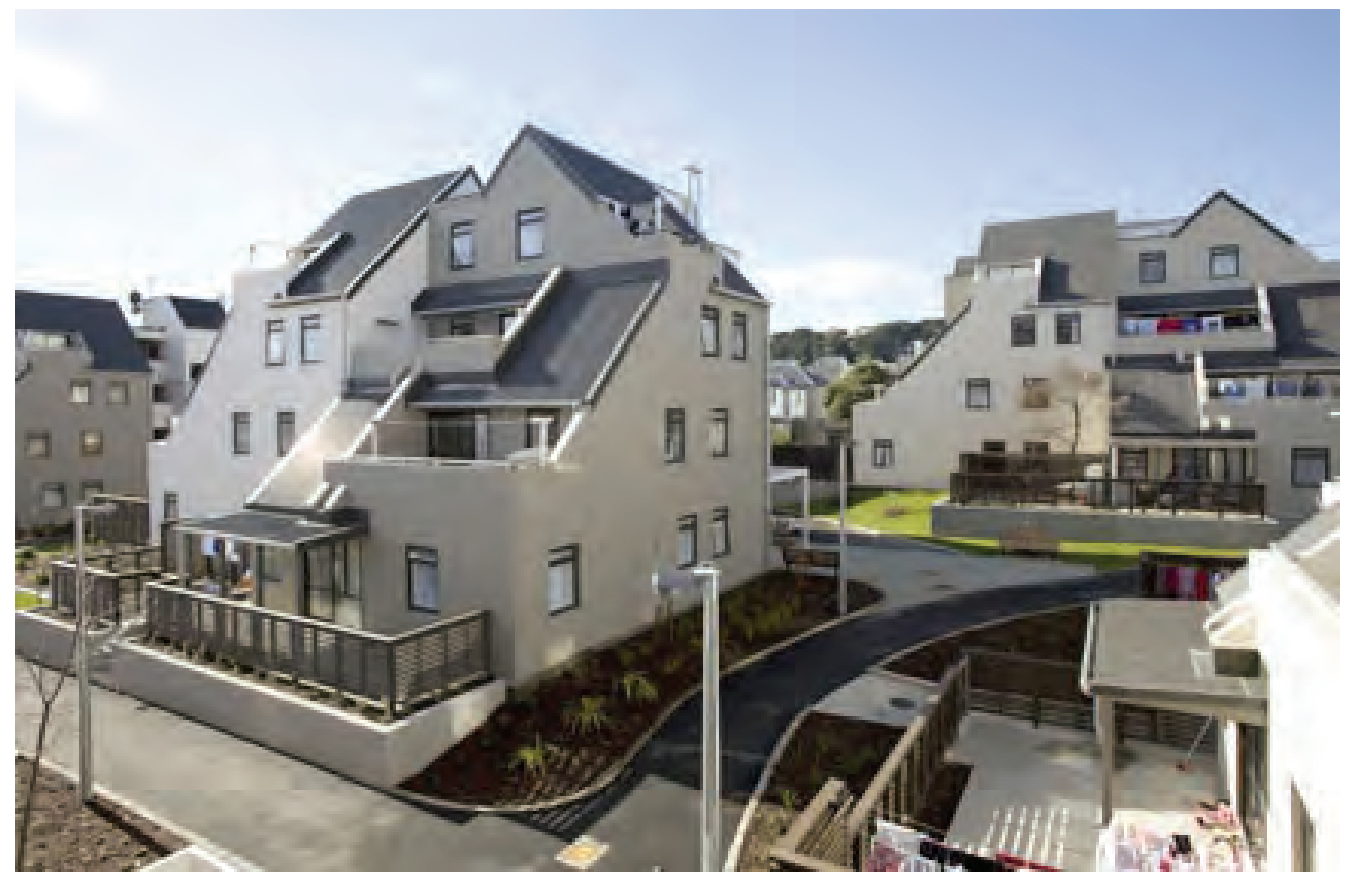

Figure 5.6 - Graph showing Te Ara Hou's proportionately high number of two and three bedroom units compared with WCC's portfolio as a whole.

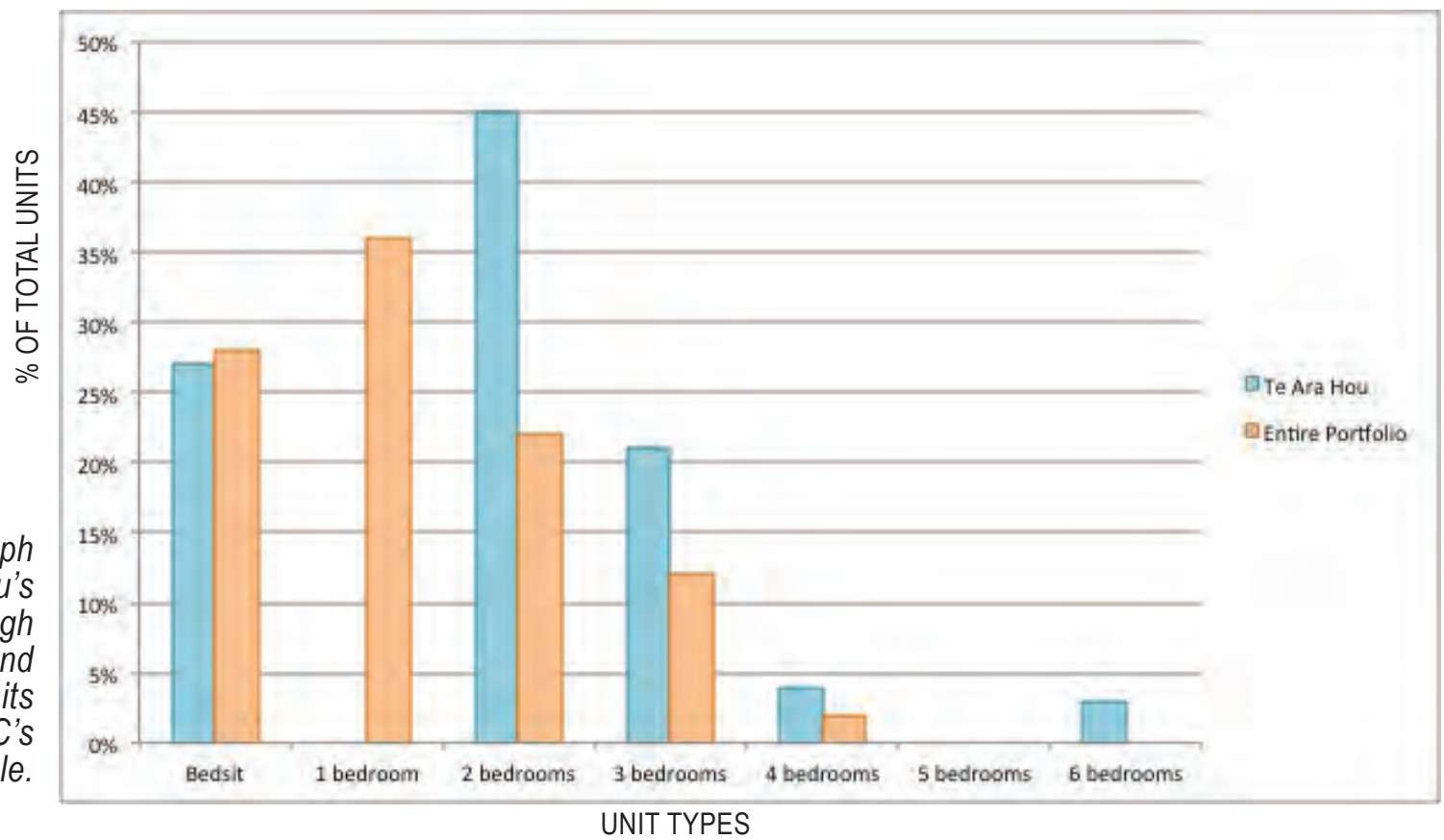

The complex is also unique within WCC's housing portfolio as a whole as it contains more two and three bedroom units than most other complexes (see Figure 5.6 above). This classifies Te Ara Hou as a 'family complex' within the portfolio. Novak and Middleton's initial scoping exercise of the complex in 2008 resulted in a concept design report which identified the following key areas for attention in an upgrade:

- Thermal and acoustic insulation is required to be installed.

- Kitchen and bathroom layouts need modernised.

- Multiple entries to site create security issues. Generally, legibility and way finding need attention. 
- Landscaping and definition of exterior space (including provision of some private outdoor areas for ground floor units) will dramatically improve atmosphere of complex.

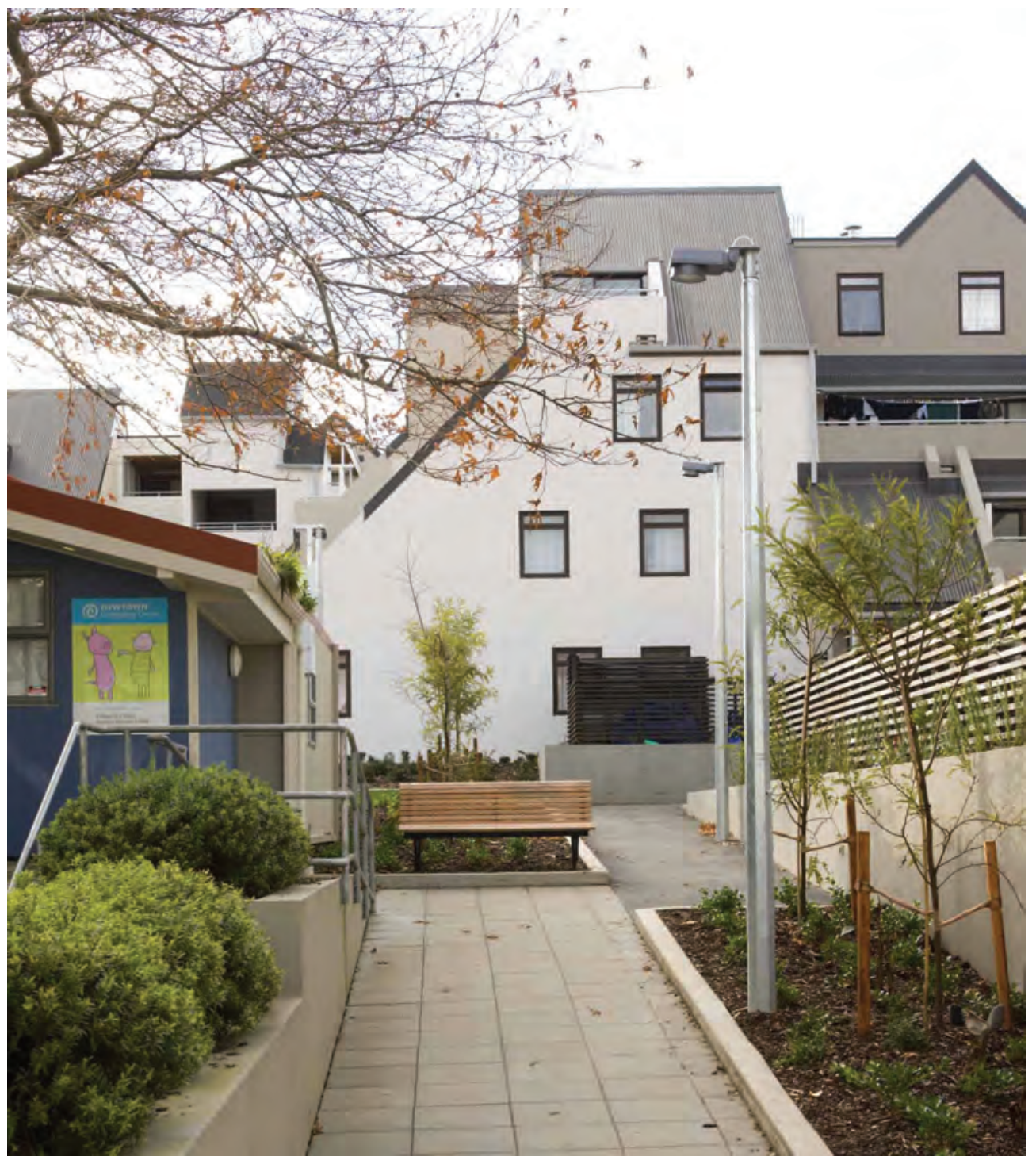

Figure 5.7 - Entrance off Daniel Street postupgrade

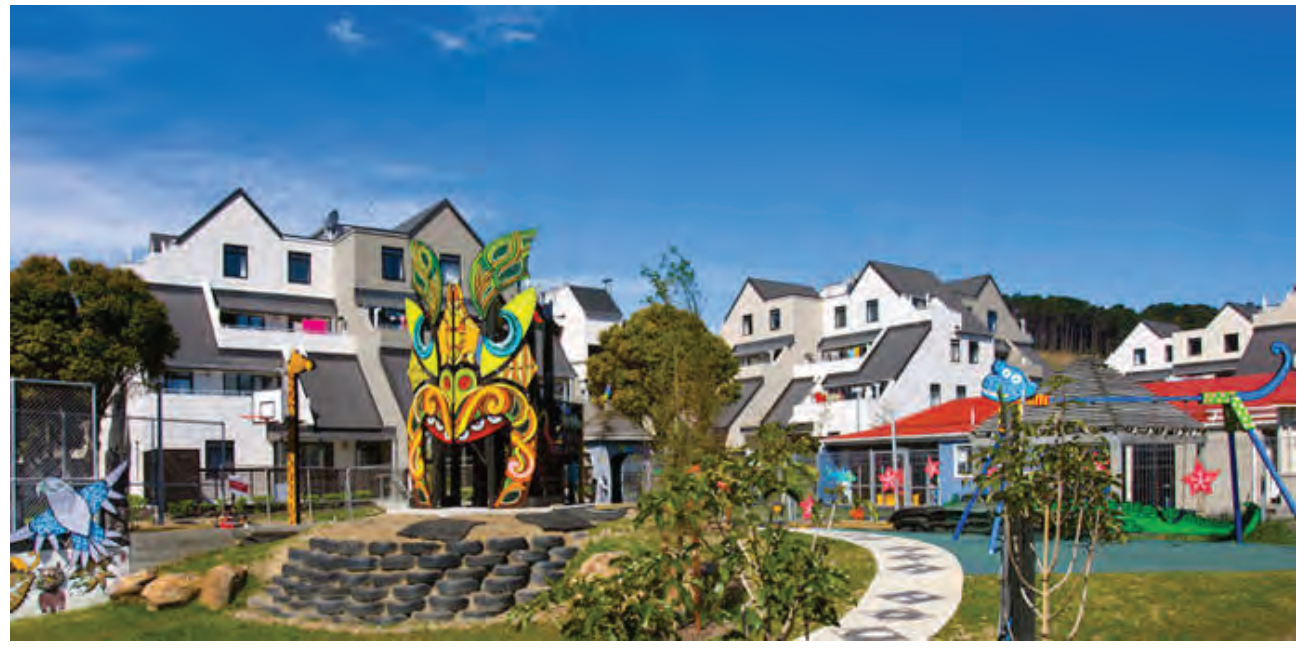

Figure 5.8 - Playground on corner of Constable and Daniel Streets also redeveloped with upgrade 
Significantly, the buildings were found to be structurally sound and no major reconfigurations of unit layouts was required (unlike many subsequent upgrades of other complexes). It was, in part, for this reason that Te Ara Hou was selected as the first complex to be upgraded as it would prove to be a relatively straightforward upgrade to begin the 20-year upgrade programme. Figures 5.2 and 5.3 give an idea of pre-upgrade conditions and Figures 5.5, 5.7 and 5.8 show post-upgrade conditions.

Novak and Middleton were appointed as the architects to complete the upgrade job in 2009/10. True to their initial concept design report, the focus of the upgrade was on improving the thermal performance of buildings by installing new doubleglazed windows and insulation, installing better waterproofing, and paying significant attention to landscaping between buildings. Spaces between buildings are now more appropriate for human occupation with private courtyards off ground floor units, public seating and significant areas of planting. This is a vast improvement on the vehicle-dominated nature of the grounds prior to the upgrade (see Figures 5.2).

The public playground on the corner of Constable and Daniel Streets also was upgraded along with the rest of the complex. This project was coordinated by the Community Action team at City Housing and a local artist who involved many of the children of Te Ara Hou in creating the artwork for the project. The upgrading of this playground alone was a considerable participatory project, and the benefits of this tenant participation has infomed much of the positive evaluation made of WCC's existing procees in 6.2 .

The upgrade of Te Ara Hou won an NZIA award for sustainability in 2010 and has been largely praised as a very successful start to the Housing Upgrade Programme. 


\subsection{Participatory exercises at Te Ara Hou}

\subsubsection{Introduction}

This sub-chapter documents my own participatory exercises undertaken at Te Ara Hou over a two-month period of field work (September and October). Three separate lines of enquiry were followed:

1. Semi-structured interviews

2. Small design workshops

3. Discussions over drawings

Selection of these three lines of enquiry has been informed by three factors. Firstly, interview and workshop methods have been directly informed by the ASF workshop in Kenya. They were selected to test their applicability in a different context. Secondly, the same two methods (interviews and workshops) are included as they are participatory tools that are not already employed by WCC in their approach. Interviews explore upgrades with tenants one-on-one, and design workshops explore issues spatially; two characteristics that WCC's participatory approach does not currently include. I am, after all, seeking to find out if there is 'scope for widening tenant participation in the Housing Upgrade Programme' (lead research question). Thirdly, the final line of inquiry (discussions over drawings) is largely responsive to the two other methods that had gone before it. This line is less informed by WCC's or ASF's participatory approaches, but finds some basis in the literature.

This two-month programme of participation was partly planned in advance, as outlined above, although I was sure to remain flexible in running the programme in light of the best practice principle that processes 'can't be neatly packaged or predictable' (see 2.3.4). I now elaborate on the process and findings from each line of enquiry separately.

\subsubsection{Semi-structured interviews}

The semi-structured interview approach was appropriate to begin the 'field work' phase of the project as I needed to hear a range of views within the Te Ara Hou tenant group on the results of the upgrade (and WCC's participatory process, for those who took part). It also provided enough flexibility for the beginnings of a 
design proposal to emerge, as I prompted tenants with questions like 'what are some of the things the upgrade missed?'

Semi-structured interviews are conversations between an interviewer and interviewee/s that are partly guided by an interview plan yet flexible enough to venture into areas that are particularly significant to the interviewee/s. John Zeisel (2005) and Nick Wates $(2000,2008)$ have provided much of the guidance on this 'focused interview' technique that strikes a balance between structure and openendedness. See Appendix A for the 'interview guide' I followed.

17 semi-structured interviews (herein, interviews) were conducted over a 3 week period at Te Ara Hou ( $25 \%$ of 67 households interviewed). Selection criteria were not strict as I was limited by whoever was home at the time of my door-knock and who, of those who were home, were interested in participating. I visited at different times of day and on both weekdays and weekends to catch a range of schedules, and all areas of the complex and a good range of demographics were eventually reasonably well represented. I recorded findings by taking brief notes during conversations, then writing a day of interviews up in more detail following the visit.

Full records of each interview are appended to this thesis (see Appendix B). More succinctly, however, the following word-clouds are intended to give a quick overview of responses to interview questions 'at a glance'. Word-clouds are a text-data presentation method that encode word frequency information via font size. Usage of word-clouds is diverse, ranging from casual users engaging the tool as a toy to create irrelevant images to journalists creating insightful visuals of presidential speeches. Some researchers are engaging word-clouds as a data-analysis tool as I have done here (Viegas et. al. 2009). In a word cloud, larger font sizes indicate more frequent responses (note: in these examples, font sizes are only comparable within one question; they do not relate across different questions). Positions of words/phrases in a 'cloud' are determined at random. 'Clouds' are automatically generated via free web applications, into which

[1] Word-clouds here have been generated at www.wordle.net one pastes text which gets automatically generated into a graphic of that body of text. ${ }^{1}$ The process followed to create a graphic for one question is outlined in more detail in Appendix C. 


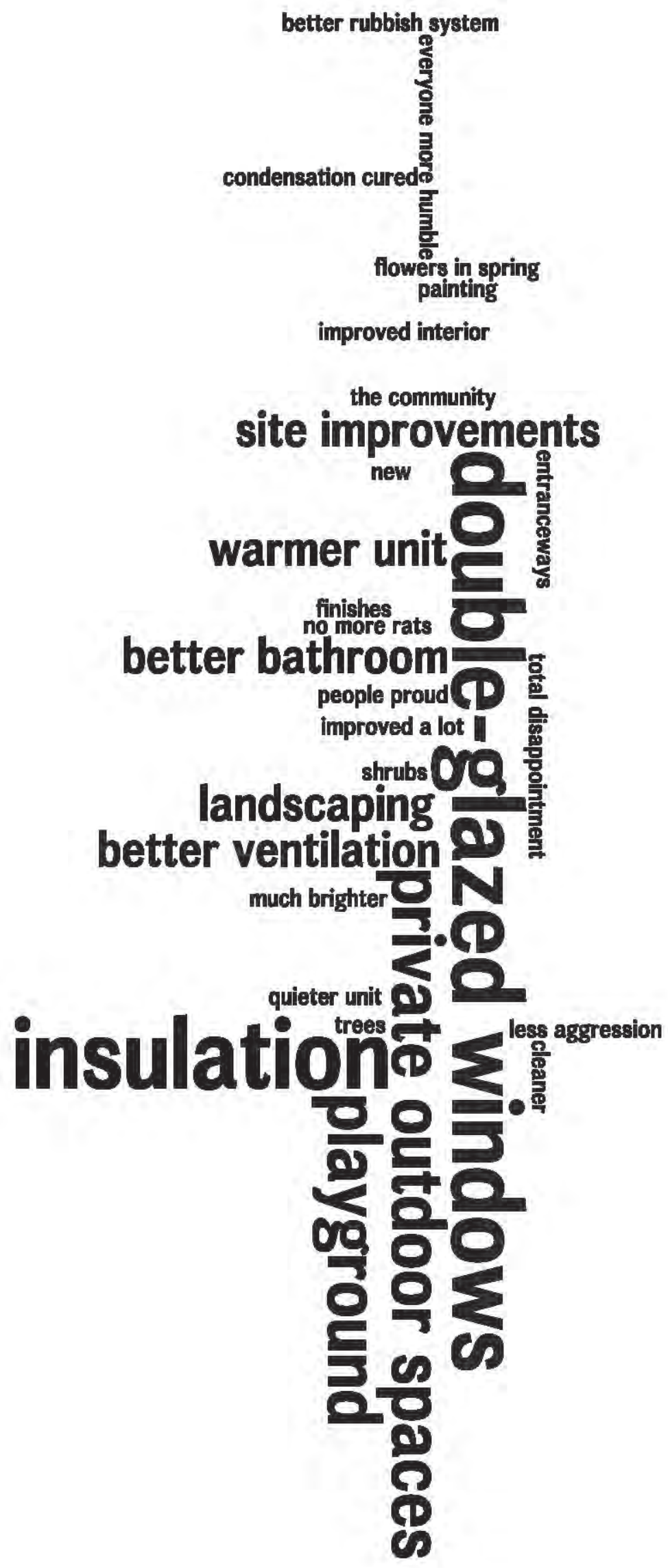




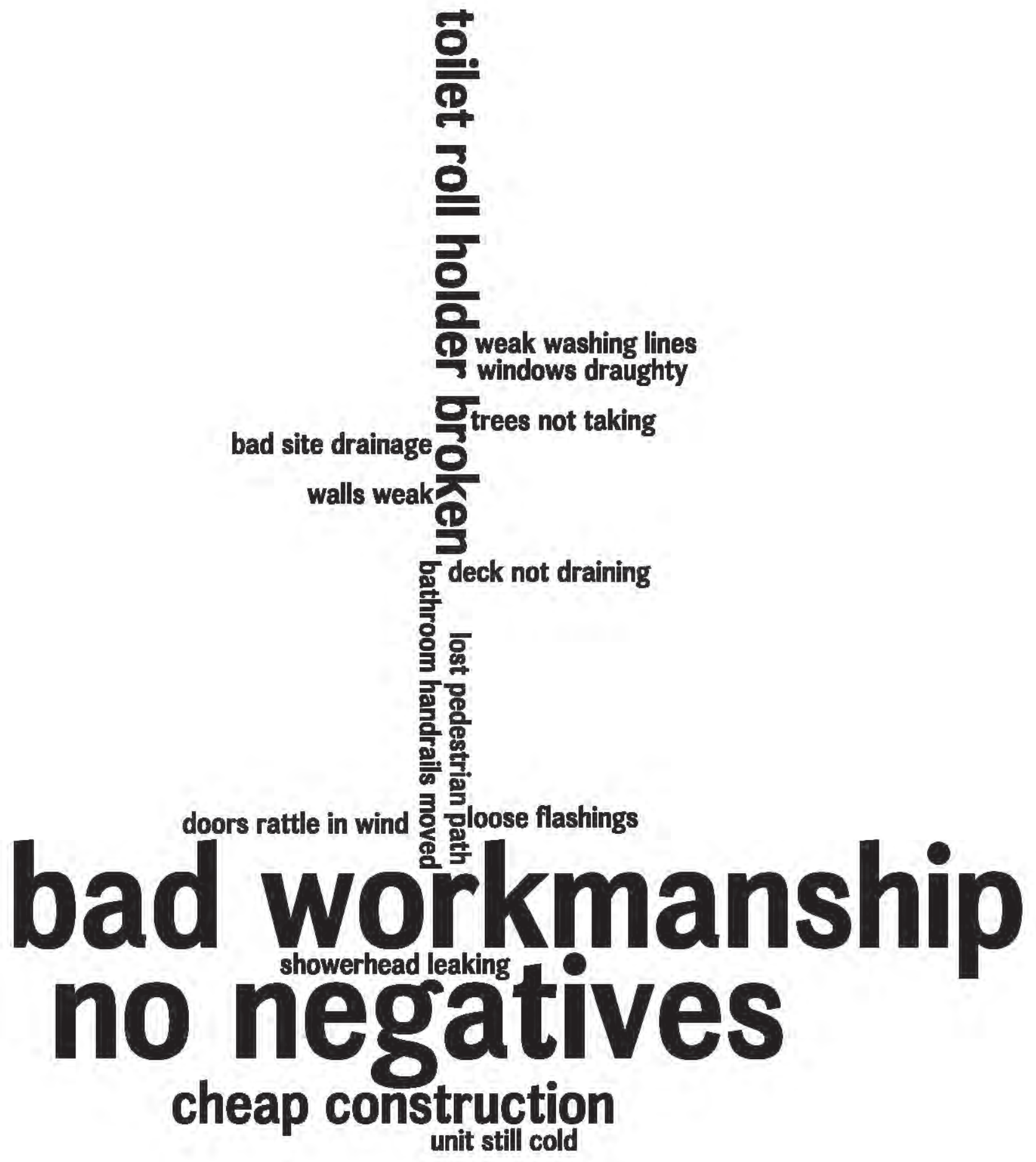


Question 3. Were you involved in any of WCC's tenant engagement prior to the upgrade? If yes, how was it? [11 responses]

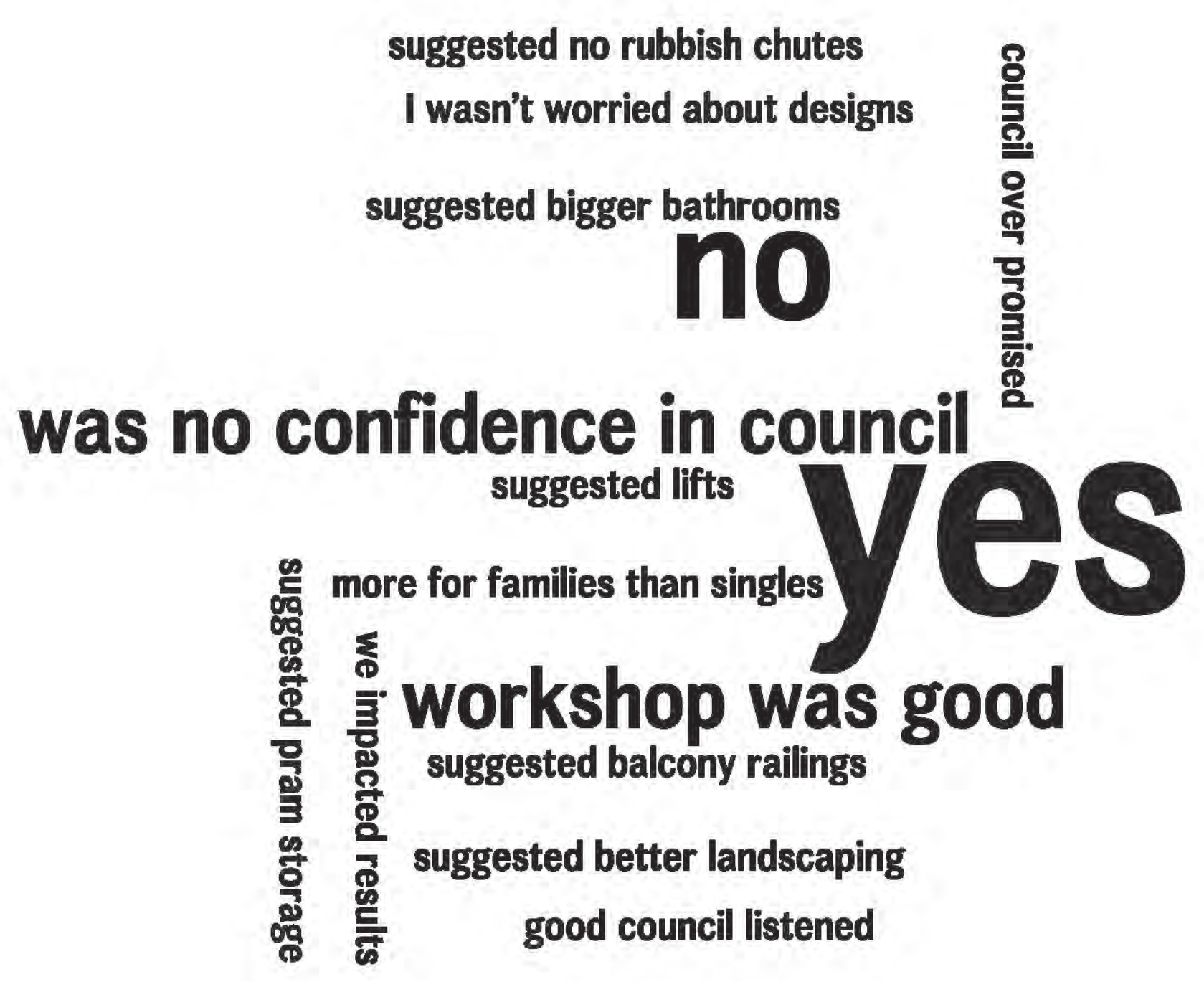




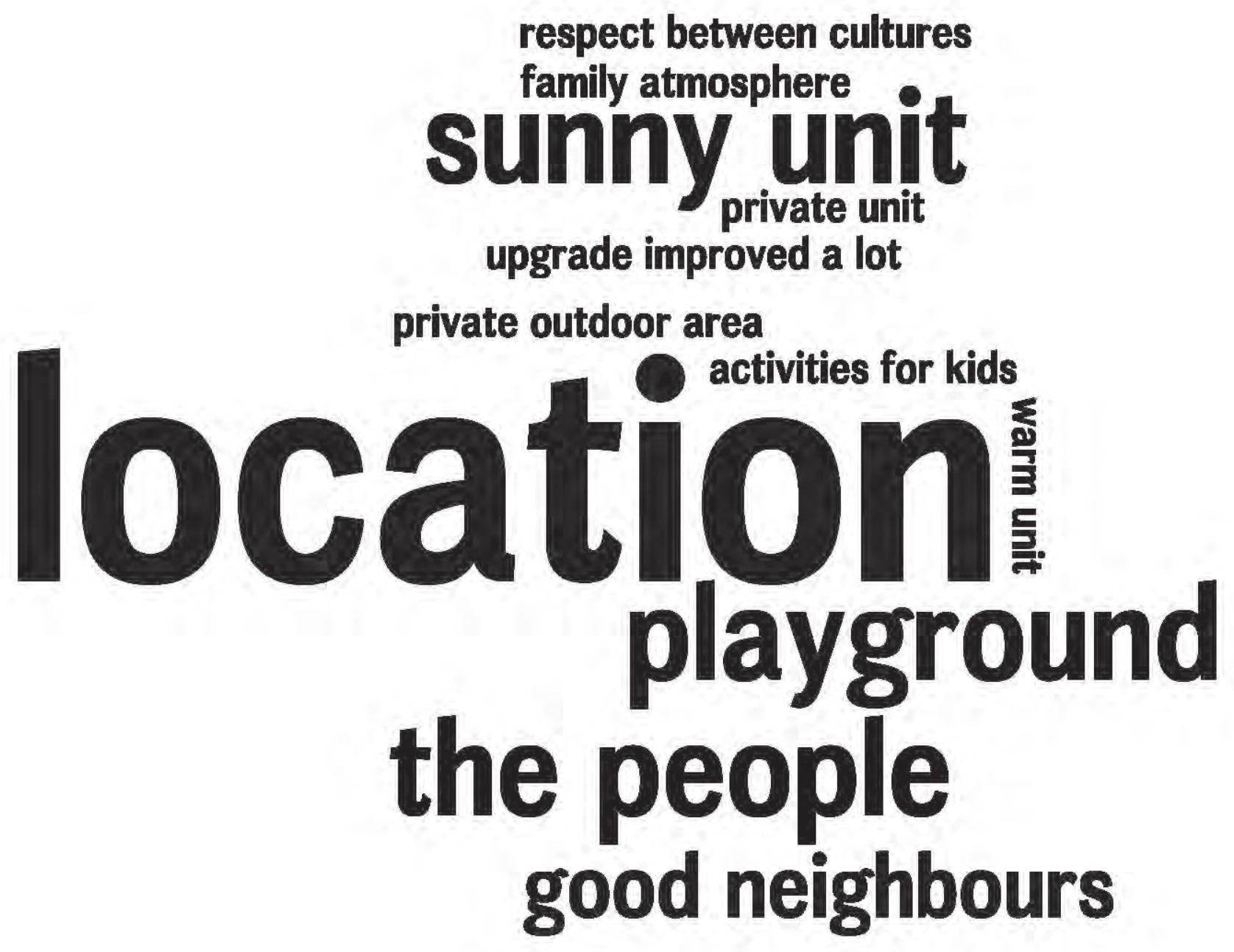




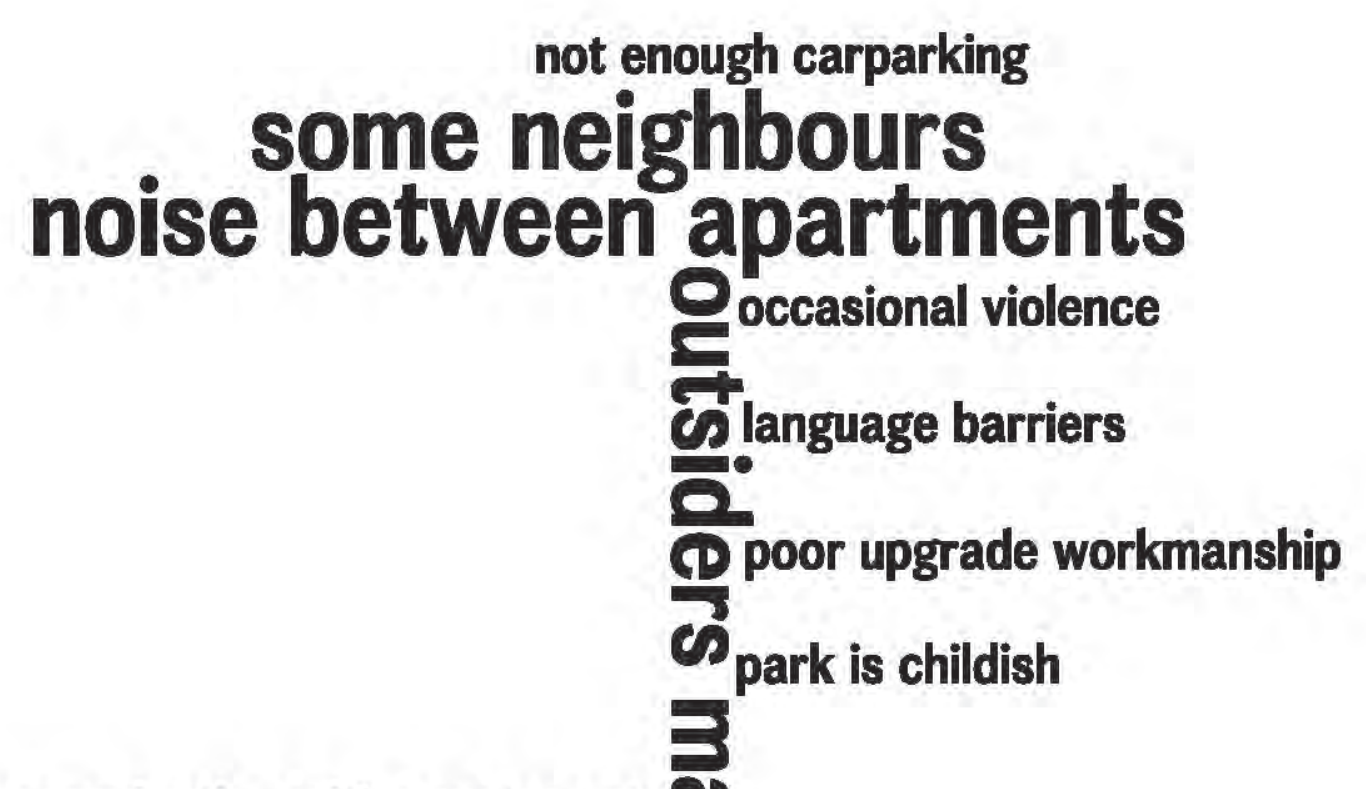

difficult communicating with new tenants

\%

ㄹ.

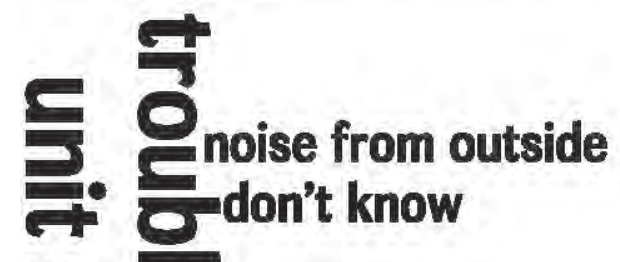

유ำกthing

号

Q) 


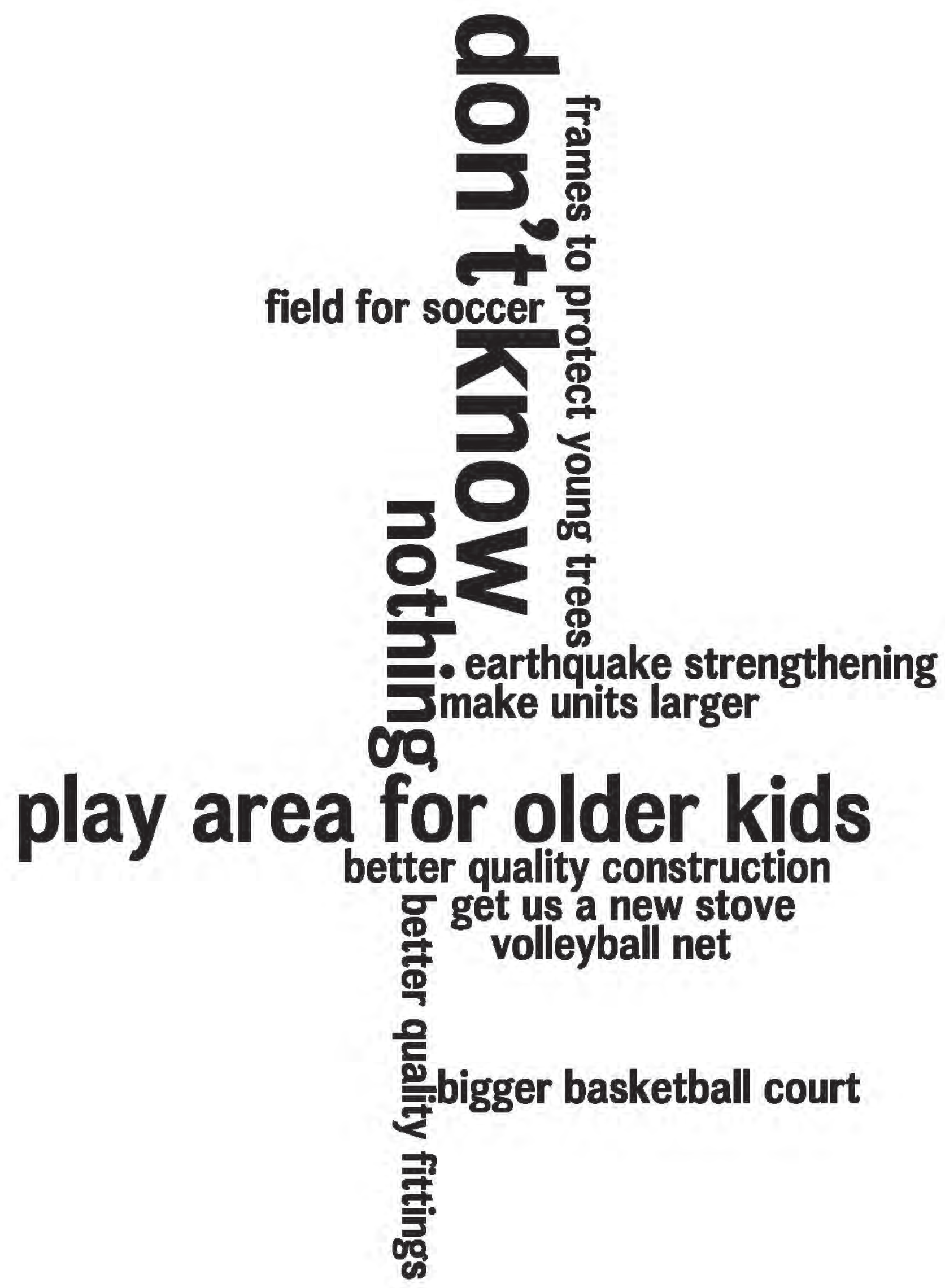


Question 7. How well do you think Te Ara Hou fits with its surroundings? (Social networks, thoroughfare through site, playground use, etc) [6 responses] *

${ }^{*}$ As this proved to be a difficult question, I avoided it in many of the later interviews. It didn't seem to fit the direction many of the conversations were generally tracking, as I had wrongly assumed it would.

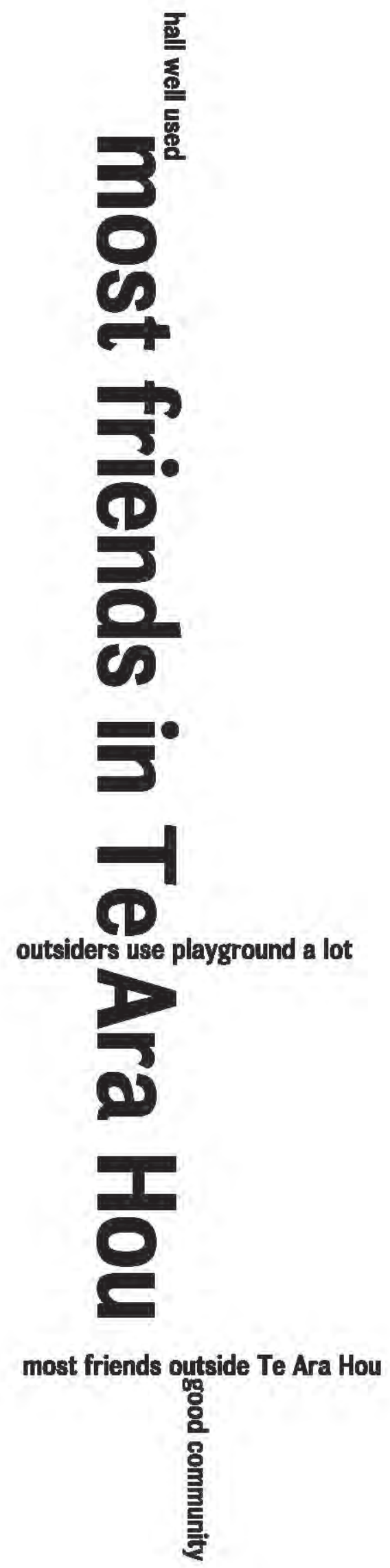


* Any points raised throughout the interview that fell outside the previous 7 'questions' have been grouped into this category. This word cloud is a representation of full text responses (not aggregated, as for the other questions). These results could not be aggregated as discussions covered such diverse material that common themes could not be found or were negligible. Interesting to note that 'people' was the most frequently mentioned word.

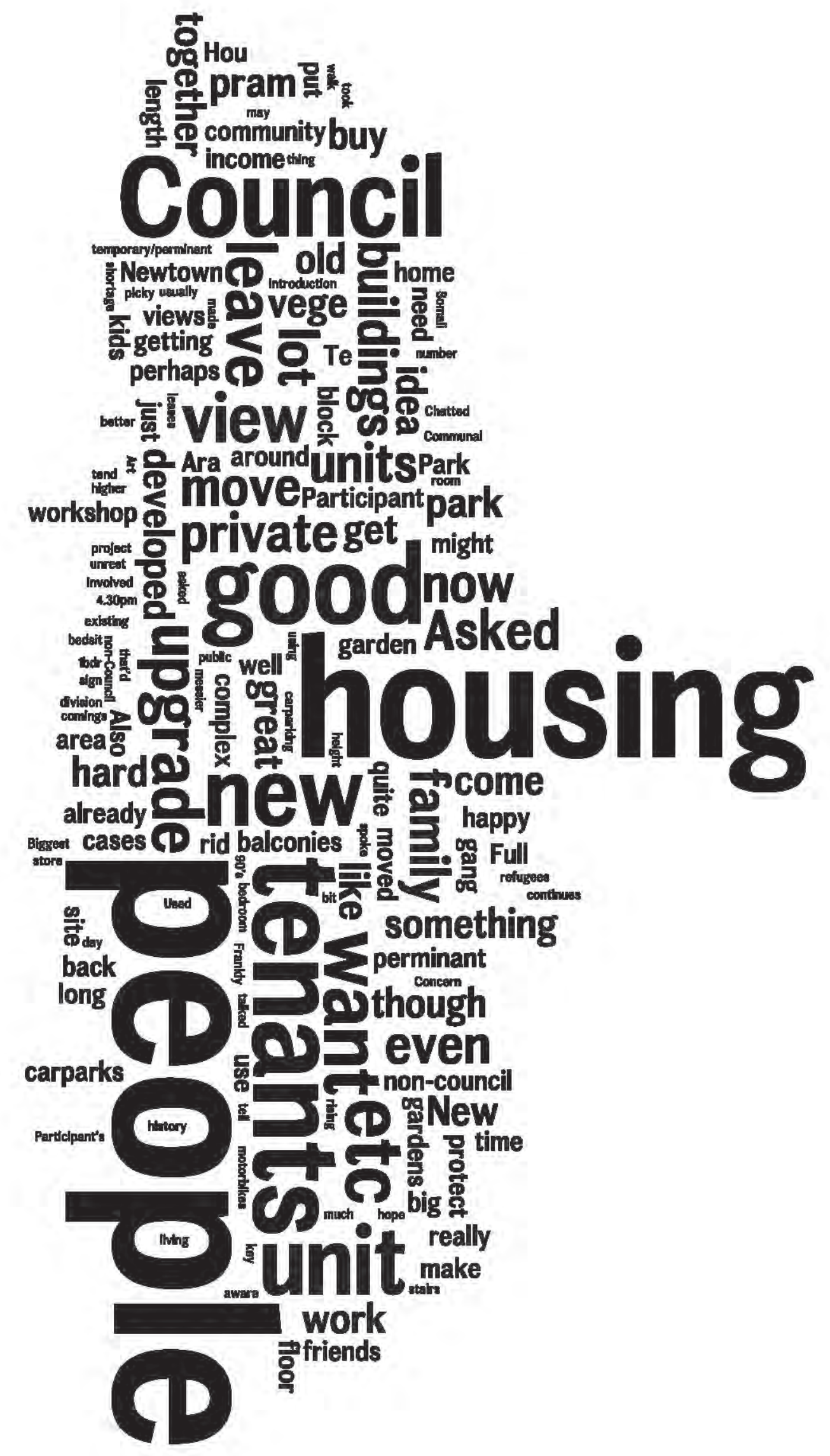




\section{RELEVANT FINDINGS}

The interviews revealed the diverse range of opinions and perspectives within the group of participants. Some participants were very forthcoming with ideas for changes to the place, while others focussed on their past experiences at the complex. Issues raised in interviews ranged from broad social concerns to practical maintenance issues. They proved extremely useful for my study in a much broader way than simply beginning the process towards a design proposal for the site. They gave me all important insights into the wide range of cultures, mind sets, world views and dreams of people living at the complex.

64 The park looks a bit childish.. It's for younger kids. We use it too ... I practice netball on the basketball court sometimes, and it's a bit childish with the giraffe on the pole."

Participant $\mathrm{M}$ (13 years old)

6 Since the upgrade, everyone is just more humble now. There's way less aggression. People are proud to be living in these flash apartments. Everyone is just positive now - people are actually proud to invite their friends over now, "you should see my place..." they say. There's o urine smell in the stairs ... Kids friends ask them "are you rich or something?!"

Participant K

4 [in response to my idea of new housing on the site] Great idea! ... This could be more Council housing for people on the waiting list ... or it could be new housing for people to buy - because people here get really dependant and need something to move on into. Or it could even be private housing, that'd be cool. Council could make some money!"

Examples of responses in semi-structured interviews. See Appendix $X$ for full records.

Participant I

It is also worth discussing findings regarding the recent upgrade of Te Ara Hou before moving onto those findings most relevant to a design proposal, as tenant feeling on work that has been done so far had an effect on their ideas for further changes. Apart from some recurring concerns around poor workmanship 
and cheap finishes, feedback from tenants on the upgrade was overwhelmingly positive. The most common reported improvements were the improved thermal performance of units (new insulation, double glazing and ventilation) and a generally more positive atmosphere at the complex (less aggression, more pride in complex, good landscaping). An overwhelming positive feeling towards the recently completed upgrade meant that suggestions for further improvements, that could prompt a design response for my project, were less forthcoming.

That said, many of the findings from interviews could directly contribute to 'a proposal' (5.2). I heard repeated disappointment that some of the new young trees were being continually vandalised, and tenants wanted this to stop. Through interviews, I identified the age group of teenagers between around 12 to 15 years old felt that the complex lacked spaces they identified with. The communal vegetable gardening area works very well for those who use it, and some felt more provision of planter boxes would be well-received. Finally, when prompted by a suggestion from me about new housing being developed on the site, there was some support for the idea but concern about the lack of space for new housing given the current density of residents.

Interviews were my first and most successful line of enquiry. They were logistically straightforward for me to carry out as a researcher working alone. I found them to be a successful method to engage less sociable people (who, for example, might not have otherwise have come to an event with other unknown tenants) and a great way to discuss matters in depth, although they were particularly time consuming given that one can only interview one (or perhaps up to 3 ) people at any given time. The interviews provided me with the broad overview of the complex that I needed to understand the context. That very general nature, however, did not allow for the kind of exploration of spatial proposals, which led me to begin the next phase of work - small design workshops.

The method of processing responses into word clouds proved useful at not only presenting material in a way that can be understood at a glance, but also as a more objective 'check' of my subjective interpretation of findings. It was interesting for me to note, for instance, that as many people had 'no negatives' to report from 'what the upgrade missed' (question 2) as there were complaints about the poor workmanship. I was left with a strong impression of the common complaint of poor workmanship as those individuals complaining about it were generally empassioned and discussed the issue at great length, whereas 'no negatives' was 
often said lightly (happily - and less memorably). Word clouds proved to be a good check of the numbers that ensured I didn't only carry forward those findings that were most memorable for me.

In all, the method of semi-structured interviewing at Te Ara Hou seemed to be as successful as the day of 'walking and talking' (casual discussions) was in Kenya (see 4.3.1). The flexible format allowed participants values and aspirations to be revealed. Whilst the one-on-one contact with participants was somewhat timeconsuming, it was not prohibitively so and could therefore be seriously considered as a valid method in WCC's process. 17 interviews represented a good sample size ( $25 \%$ of complex), and they took approximately 20 hours in interview time and subsequent processing. Paid to a researcher on an hourly rate, this cost would not be excessive.

\subsubsection{Small Design Workshops}

This method is directly informed by the model-based sessions at the ASF workshop (see 4.3), which proved to be successful in that context. I was ready to test a participatory method that was more interactive and spatial than the interviews. This more 'constructive' line of inquiry had the explicit intention of being a catalyst for beginning the design process - and even generating design options - as per the Ministry for the Environment's advice that design workshops are an effective, accessible way to rapidly investigate and visualise development options with 'lay people' or non-designers (2009:32). I built a 1:200 scale model of the Te Ara Hou site and immediate surroundings (measuring $800 \mathrm{~mm} \times 800 \mathrm{~mm}$ ) which became the focus of these workshops.
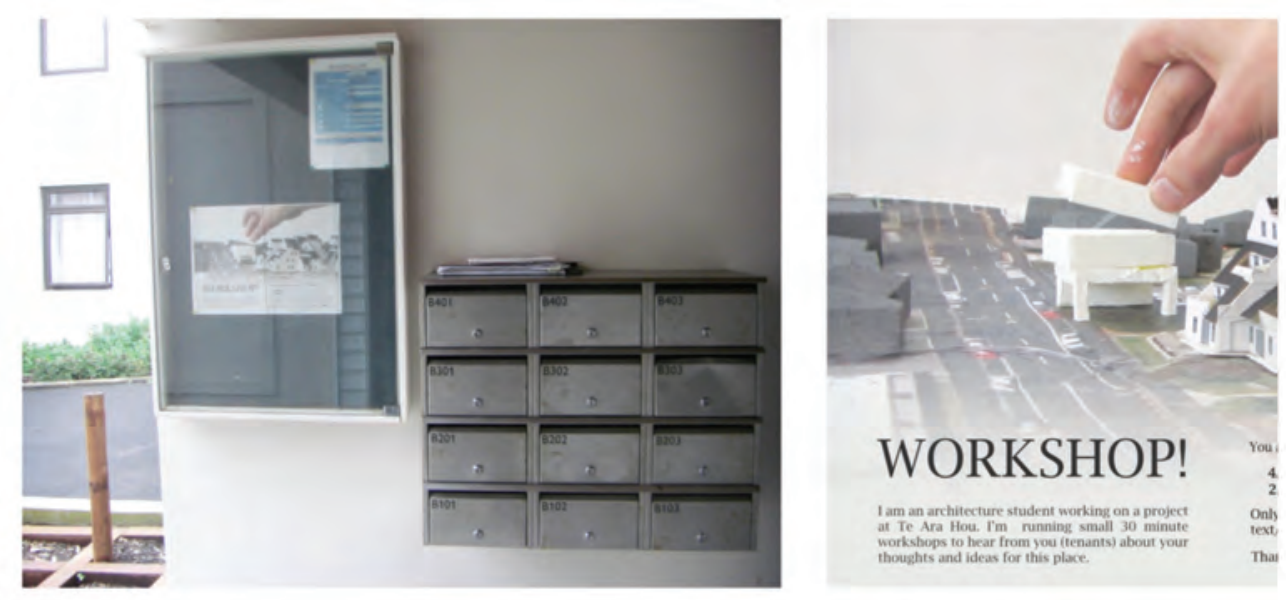

Figure 5.9 - Posters advertising workshops were pinned up around complex 
[2] See X.X for more discussion around the provision of incentives for workshop attendance.

Figure 5.10 - Tokens identified participants'

favourite and least favourite areas on the site model. X Photo used with permission of participants. $X$
I held three workshops over two weeks in the Newtown Hall (on the Te Ara Hou site) with 3-5 prearranged participants attending each session. A student colleague from the School of Architecture assisted me with each workshop, so separate discussions could break out if required. In a similar vein to the interviews, selection criteria for workshops were not strict. To seek sign-ups, I initially pinned posters up around the complex (see Figure 5.9), although a lack of response from posters prompted me to personally invite tenants who I had already engaged in interviews to participate, and this proved more successful. As a very small gesture of hospitality and gratitude towards participants, I provided tea, coffee and biscuits. ${ }^{2}$

The workshops began with an exercise to identify participants' favourite and least favourite areas around the site with small tokens - happy and sad faces. Figures 5.10 and 5.11 show the exercise unfolding in the workshop, and Figure 5.13 presents some of the annotated findings.
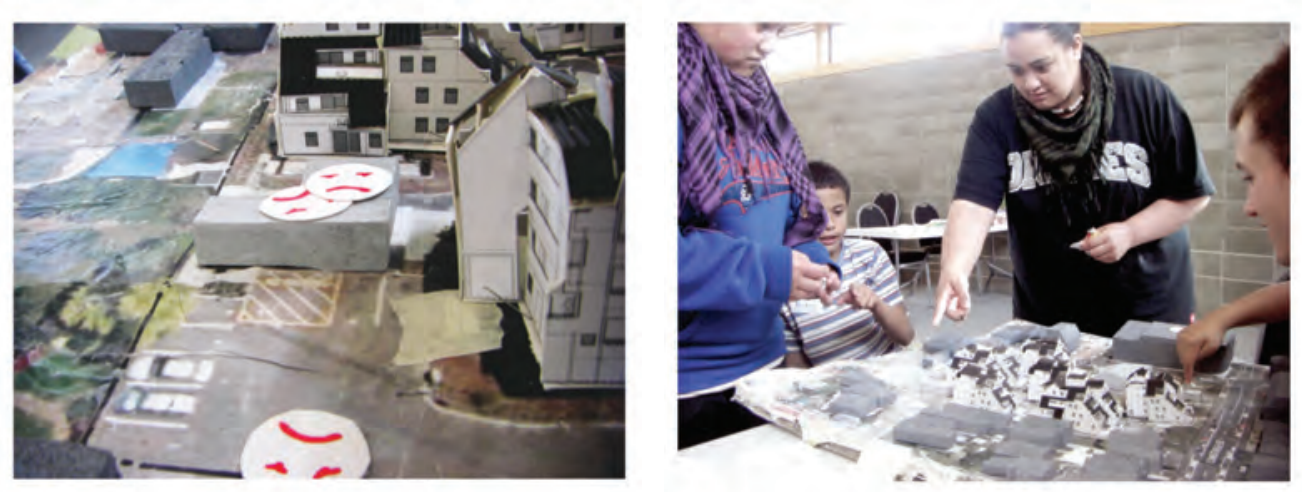

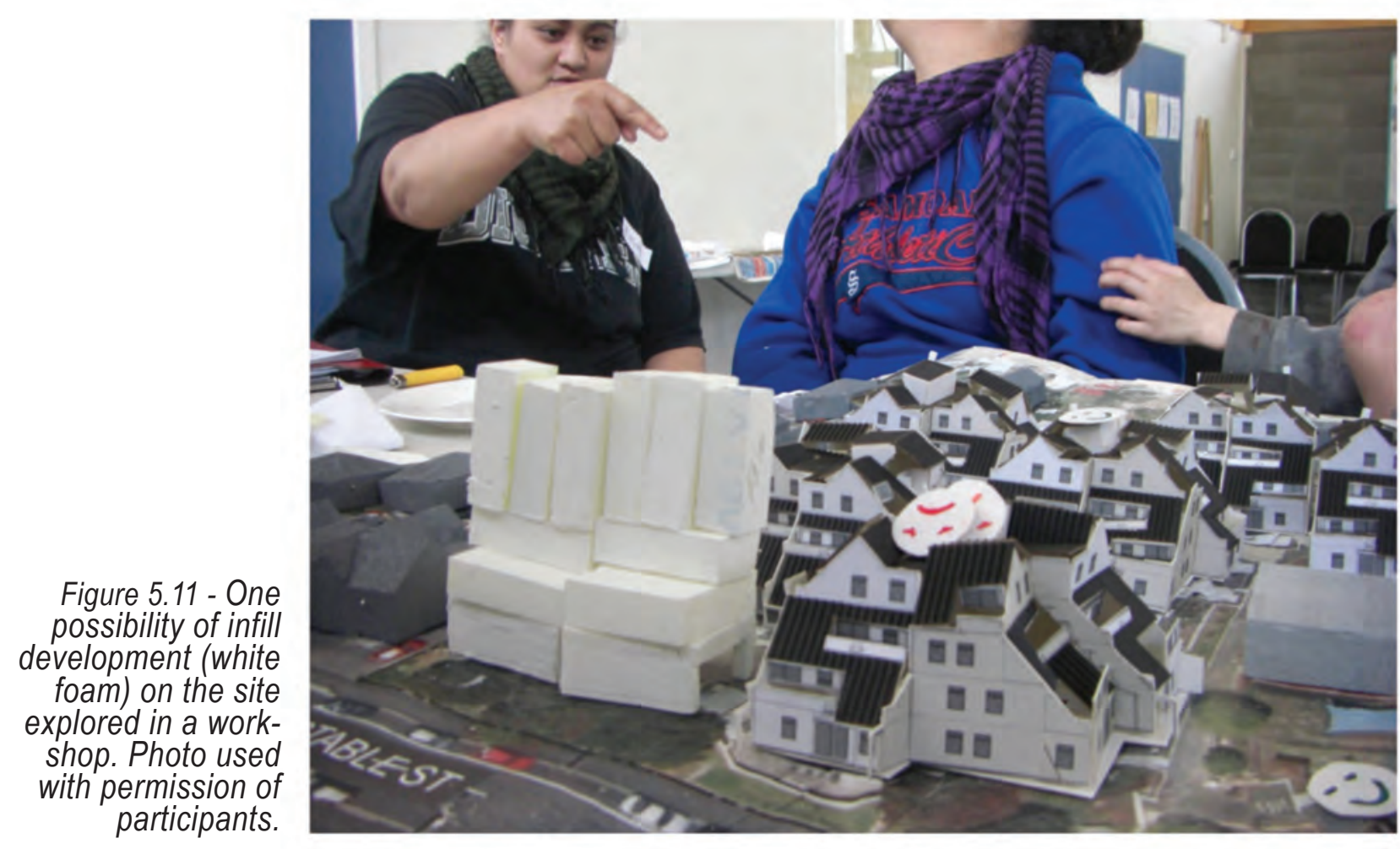

Figure 5.11 - One possibility of infill development (white foam) on the site explored in a workshop. Photo used with permission of participants. 

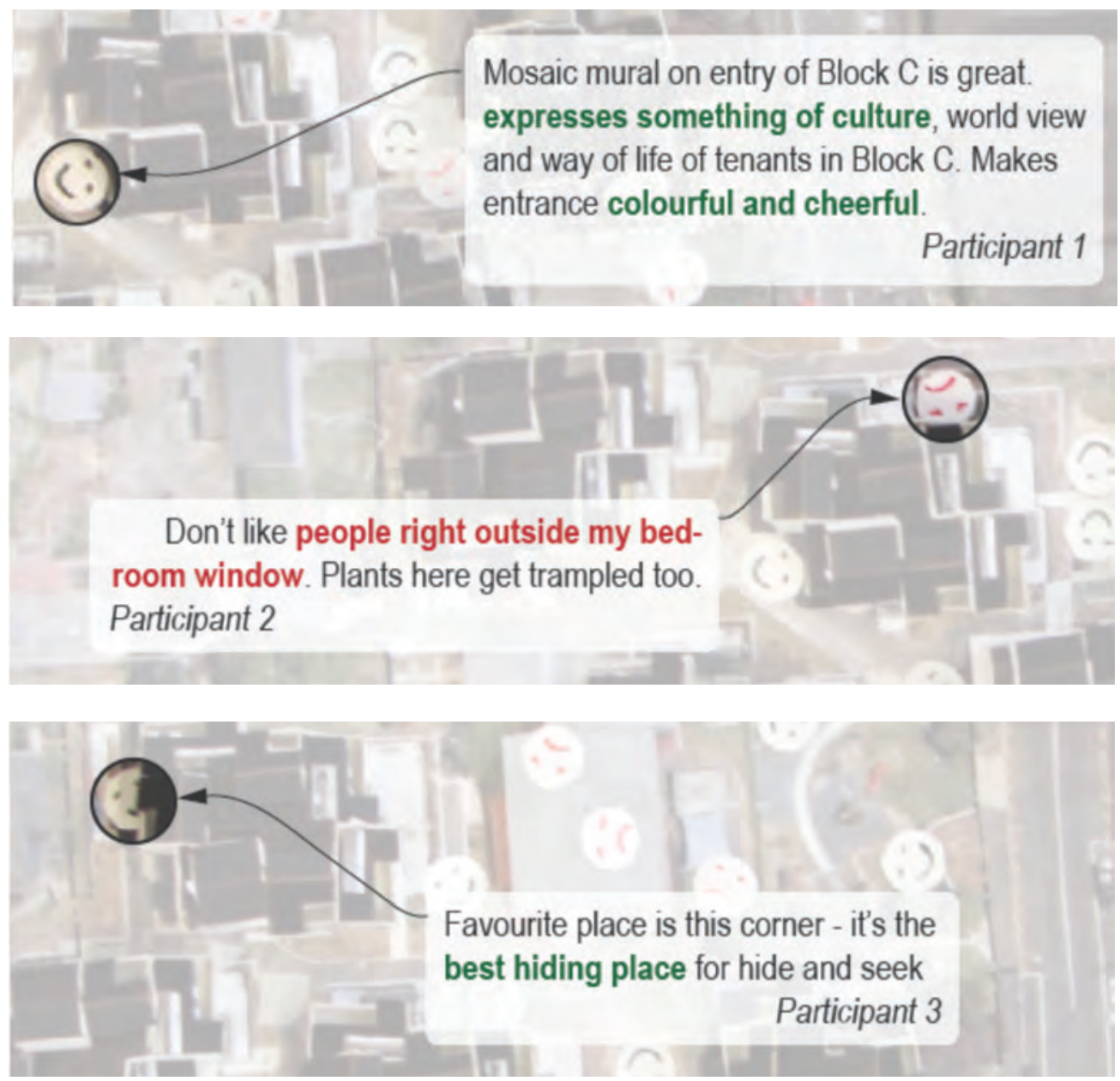

The most useful findings are summarised as follows:

Positive:

- Playground very well liked area. Attachment to place evident given tenants' participation in its formation.

- New mural on Block $\mathrm{C}$ well liked as an expression of tenants living there.

- The idea of the communal outdoor area (with picnic tables) is well liked, but tenants reported that the area is not particularly well used.

- Fenced semi-private outdoor areas very good - make lounges more private

Negative:

- Tenants generally happy with landscaping, although some disappointment about some trees not taking.

- Pedestrian link out northeast corner of site was lost with the upgrade - this is missed by tenants.

- Privacy issues with bedroom windows directly onto public areas cause some concern.

- An old laundry room in the South-east corner of site received a sad face as some tenants haven't yet seen a plan for a community room there come to fruition.
Figure 5.13 - Examples of favourite and leastfavourite areas around the complex 
I then asked participants to speculate about the best areas around the site for possible infill development. This was to begin to work towards a design proposal for the site. We explored infill ideas with one-bedroom and two-bedroom apartmentsized blocks I had prepared to scale (see Figure 5.11 for one example).

The most useful findings from this exercise are summarised below:

- From the small sample of tenants I engaged, there was a general reluctance to place new buildings on the site (tenants stating that 'there's no room', 'it's already crowded enough' 'we'd have to sacrifice something important like car parking or landscaping').

- A constructive idea in one workshop was for a community room to be placed in the old (unused) laundry room beside communal vegetable garden, including a computer hub for tenants' use.

Overall, this line of enquiry did not prove particularly successful for my work at Te Ara Hou and the main reasons for this have become clear to me retrospectively. The favourite/least-favourite areas exercises flowed well as the model proved to be an engaging medium for participants. Although findings from this exercise were insightful, it wasn't quite harnessing the model for its spatial potential (i.e. similar findings could have been obtained in a 'walk-around' exercise). The next exercise with infill building blocks, while spatial, hit a major hurdle as it was based on an incorrect assumption I had made that tenants would want to explore the idea of infill development on the site.

That this line of enquiry was largely unsuccessful, whilst being a very similar method to the model-based exercises undertaken at the ASF workshop could bring into question how transferable the ASF tools are to this context. Having considered this, I conclude that what made these exercises unsuccessful here was not the method itself, rather the content of the 'building blocks' exercise and that it did not align with what tenants were keen to explore with me. This reflection is discussed in more detail in 5.1.5. 


\subsubsection{Discussions over Drawings}

Following the somewhat unsatisfactory results from the small design workshops and sparked by interesting ideas accumulated from the many discussions l'd had with tenants by this stage, I followed a third line of enquiry: discussions with tenants over drawings. This line of enquiry became a direct example of the best practice principle that a process can't be neatly packaged or predicted (2.3.4). This represented a change in tack to further investigate possibilities for new developments on the site, as I still felt these ideas were worthy of further exploration. In a sense, this was a test of a more directive approach where, in the circumstances of this hypothetical project, the more passive approaches to participation had failed to produce the kinds of responses I required to keep the research moving. It is worth reiterating that this step is simply another test of a participatory exercise, like the interviews and workshops, and not a presentation of 'a design' per se.

This approach began in a more 'top-down' fashion as I prepared sketches of three development options montaged onto photographs. The aim of these drawings was to stimulate responses (either positive or negative) in participants. The Urban Design Toolkit explains the technique as providing "tangible evidence of expected visual effects" of proposals (Ministry for the Environment 2009:60), as an attempt to better connect with lay people or 'non-designers' in a similar way to workshopping around an interactive model. The seven drawings I took into discussions are shown on the following pages, annotated with some of the participants' comments that were provoked by the drawings. 


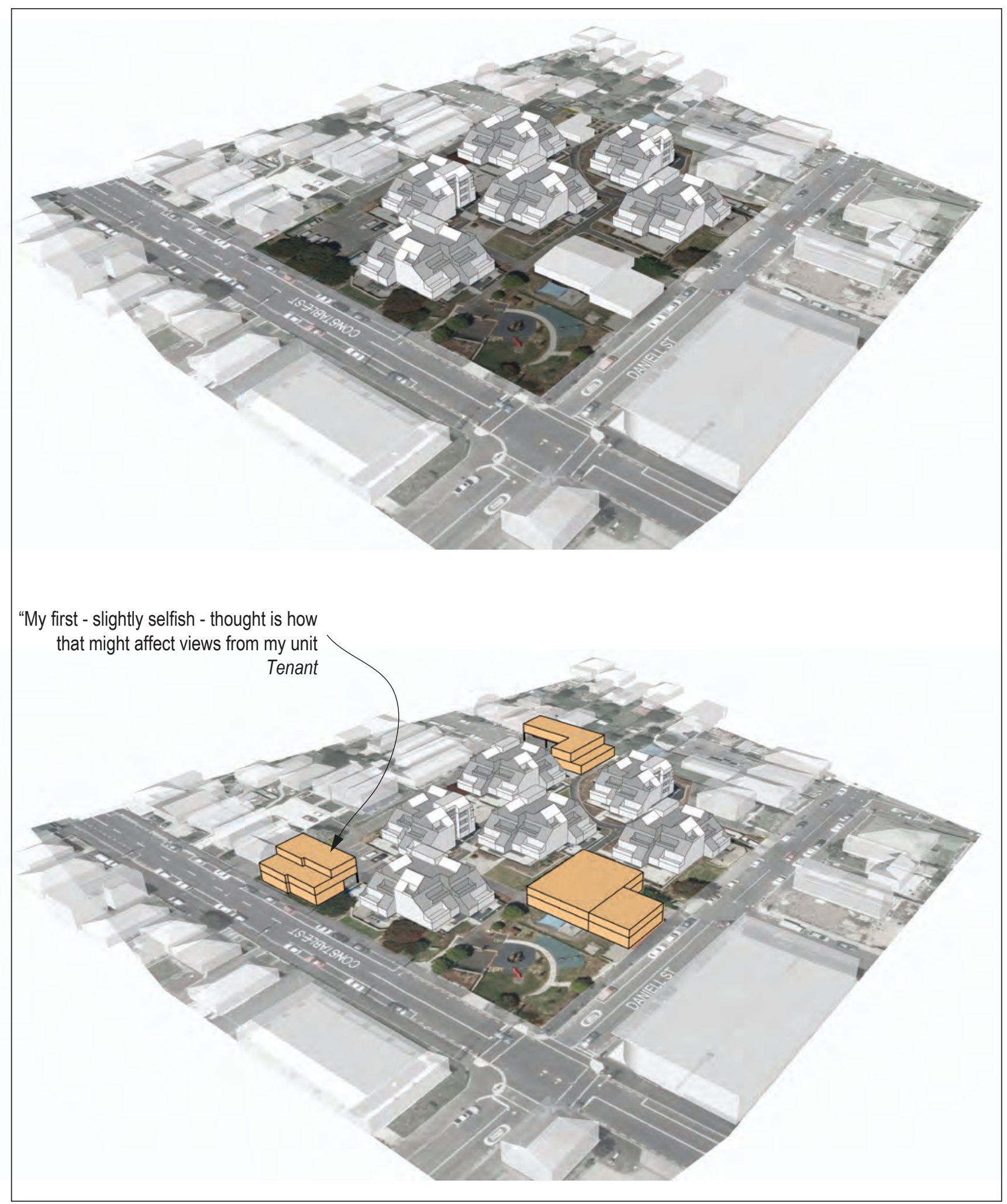

Figure 5.14 - 'Discussions over drawings' - Aerial for overview of 'proposals' (before - top; after - bottom). Annotated with a tenant's response. 


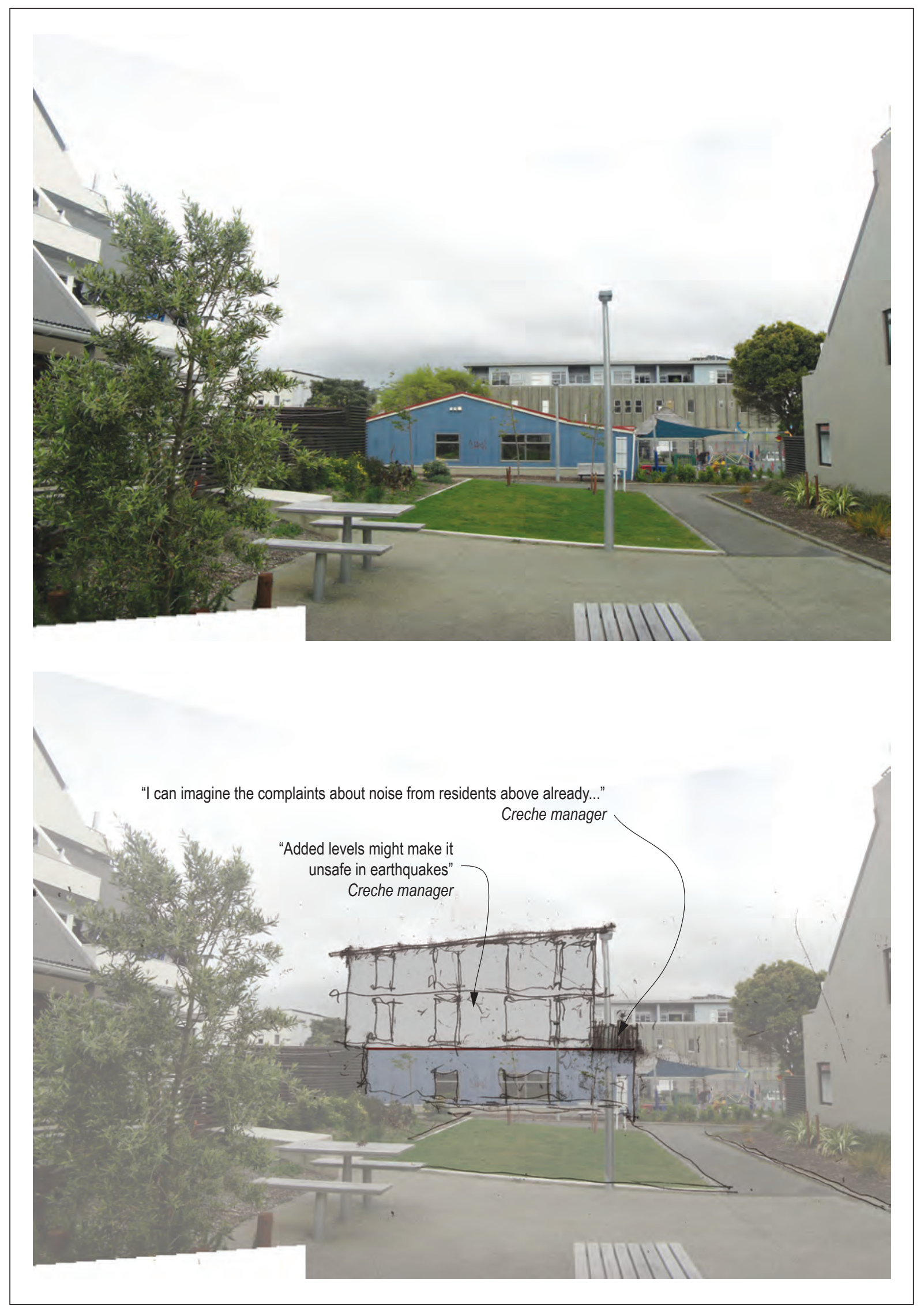

Figure 5.15 - 'Discussions over drawings' - proposal to add levels to crèche (before - top; after - bottom). Annotated with a selection of responses 


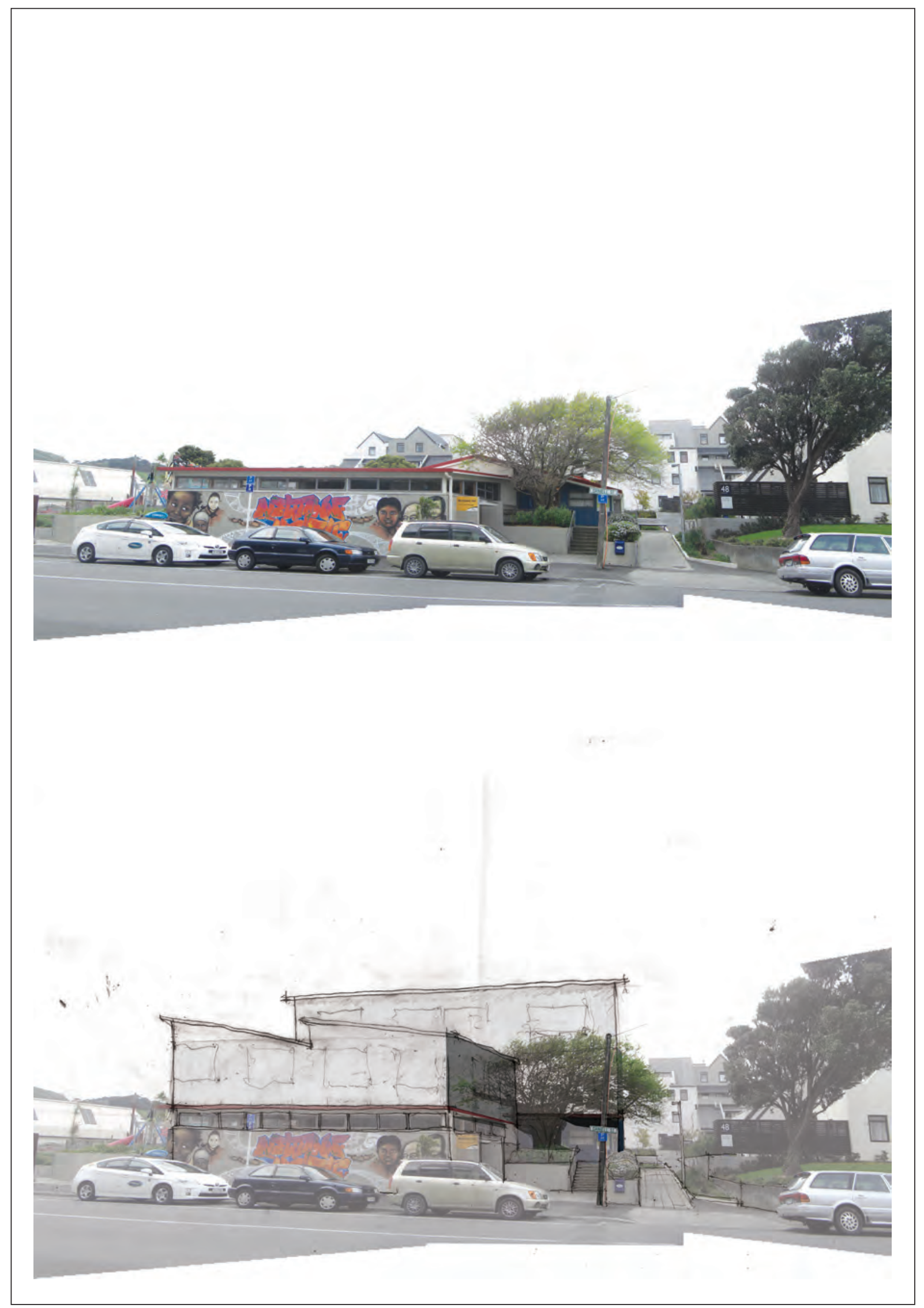

Figure 5.16 - 'Discussions over drawings' - proposal to add levels to crèche (before - top; after - bottom). 


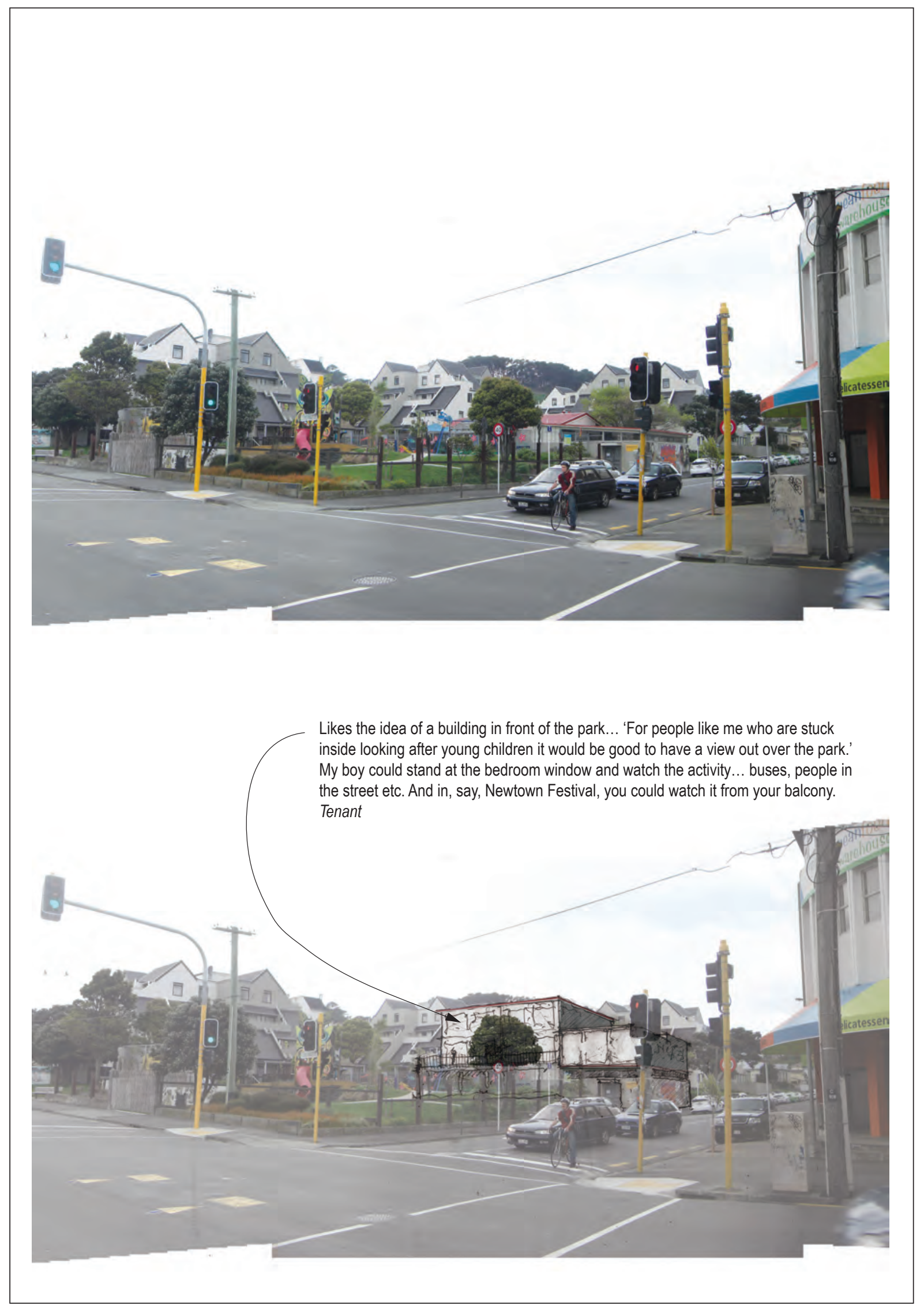

Figure 5.17 - 'Discussions over drawings' - proposal to add levels to crèche (before - top; after - bottom). Annotated with a response from a tenant. 


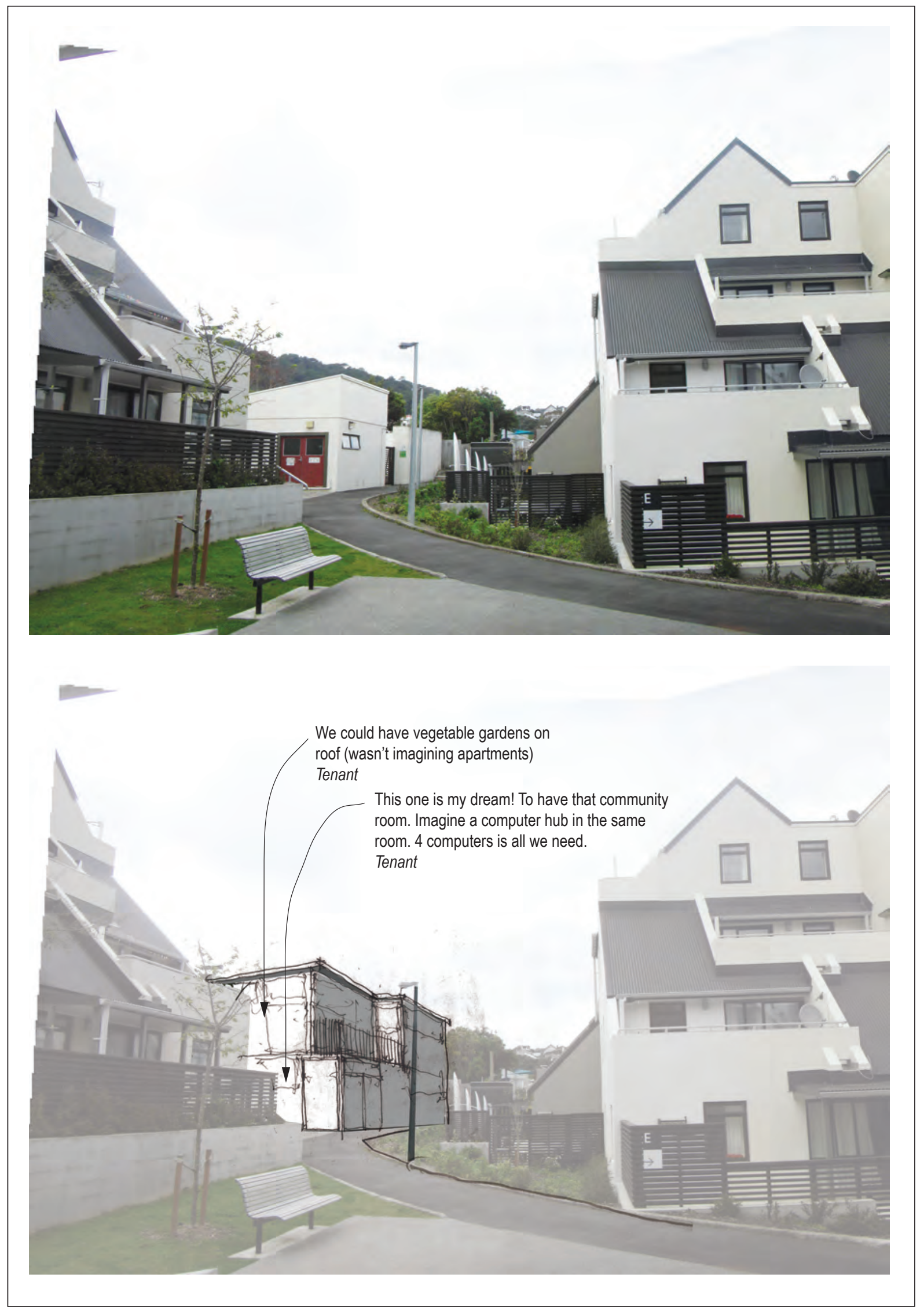

Figure 5.18 - 'Discussions over drawings' - proposal of South-east building (before - top; after - bottom). Annotated with responses from tenants. 


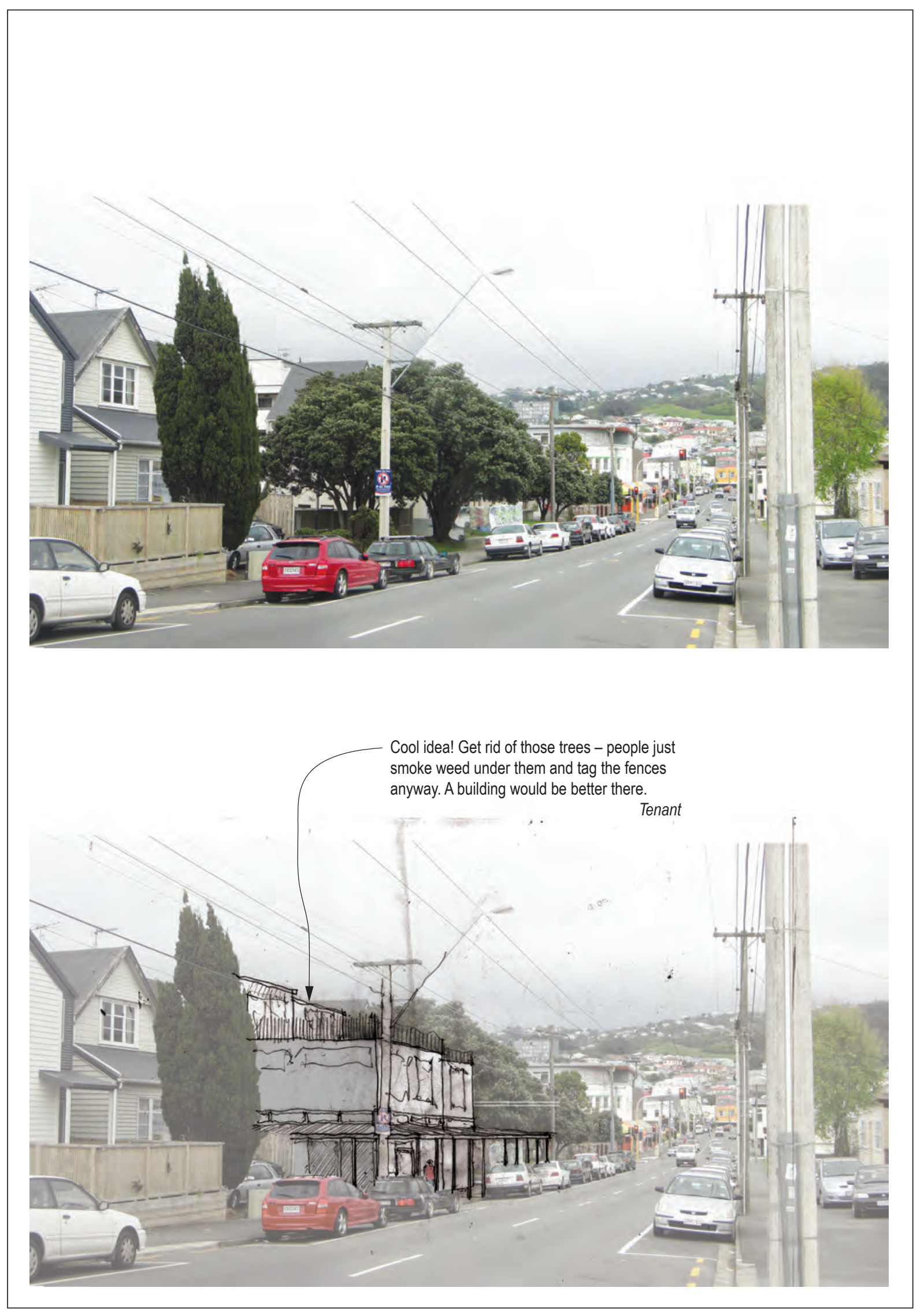

Figure 5.19 - 'Discussions over drawings' - proposal of North-east building (before - top; after - bottom). Annotated with a response from a tenant. 

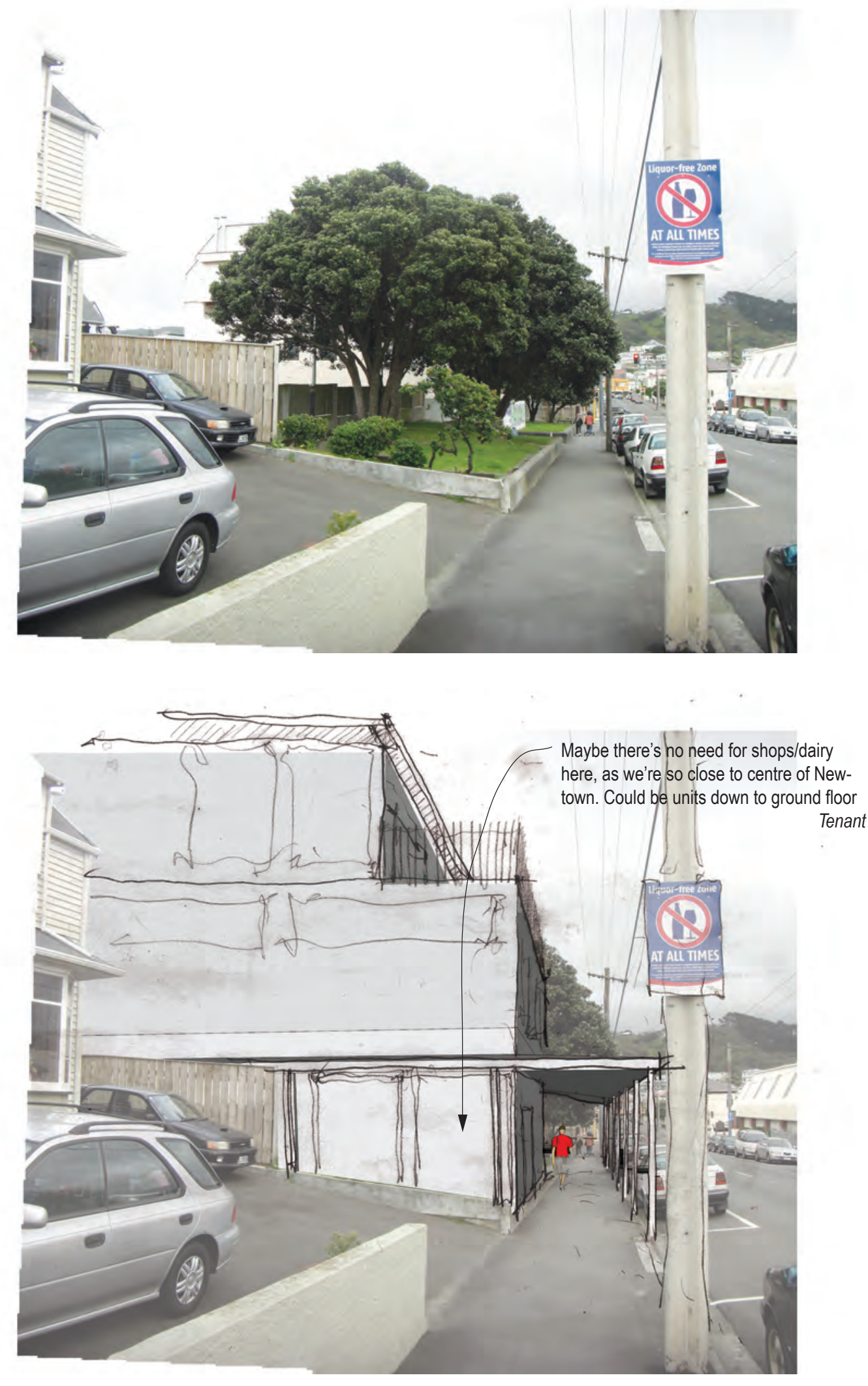

Figure 5.20 - 'Discussions over drawings' - proposal of North-east building (before - top; after - bottom). Annotated with a response from a tenant. 
The technique was successful in stimulating responses in tenants, as they were familiar with the scenes in the photographs and could easily imagine these buildings on sites with the help of visual aide. What I may have discussed with someone in an interview suddenly became more real with these drawings, and this opened up further discussion. As this step - somewhat spontaneously inserted into my programme following the first two stages - proved to be successful, I can reassert the importance (outlined by Chambers, among others) of remaining flexible in running participatory programmes, and being willing to change tact in response to circumstances at short notice (see 2.3.4).

As these discussions were as logistically simple as interviews to conduct, I was able to take the drawings to a wide range of people including non-tenants. A conversation about added levels over the crèche building with the crèche manager raised an interesting set of concerns distinct from those raised by tenants. Concern for complaints about noise from tenants suggested to be living above and concerns for child security in outdoor areas were pertinent. Another interesting perspective that arose from a discussion over these drawings was the view that a building could be a 'positive fix' to a site with negative associations like drug use and graffiti (see Figure 5.20). This is perhaps an often-overlooked benefit of increasing site coverage by adding further buildings to sites. On the topic of who new housing proposed for the site might be for, tenant opinion was mixed. Some argue diversity is desirable to avoid concentrations of certain demographics (i.e. some private units on the site could provide a good mix), while others argue all housing on the site should be exclusively for Council tenants to ensure better community cohesion.

Some thought has been given as to how this successful tool could be extended. Animated walkthroughs of a digital model of proposed interventions would be worth exploring, as they could achieve all that this method achieved and potentialy in an even more engaging way.

In summary, this third and final step in my field work revealed many more views that I could use as I moved towards developing a design proposal for the site. The aim of these drawings - to provoke responses (either positive or negative) from participants - was largely met, as I found participants could easily visualise these proposals with the aid of drawings, and were quick to share their thoughts and opinions on them with me. 


\subsubsection{Overall discussion on three participatory exercises}

\section{'UNPRODUCTIVE' WORKSHOPS}

It is with an air of relief that I look back on what, at the time, seemed like unproductive workshops at Te Ara Hou and deconstruct what went wrong in light of my now broader understanding of participatory process.

Firstly, it is now clear that much of my disappointment from the workshops stemmed from an unrealistic preconception of what they would bring to the process. My mistake was assuming that intensification of the site was an idea that participants would be keen to work with me on. I had designed an exercise around playing with building blocks on a site model to inform me of tenants' ideas and preferences for where infill development on the site could go. In every case, participants reactions were that they didn't think there was sufficient room on the site for intensification. I learnt through this process that people won't be comfortable exploring options for a suggestion they aren't on board with in the first place. I was wrongly imposing my assumption on participants. According to Zeisel, preconceptions like these are unavoidable, and he encourages researchers to use them to a project's advantage:

... researchers do not approach problems with empty minds. ... The preconceived images with which investigators begin research projects can distort what researchers see, bias explanations, and limit how concepts develop. But they do not have to ... Explicit preconceptions like these can sensitise researchers to see and to be surprised by what they see (2006:37).

Taking Zeisel's advice - considering my preconception as a sensitising tool - I did become more aware of the value tenants place on open spaces and also their overall feeling on what an appropriate density for the site might be. What I initially thought of as failed workshops did in fact teach me something of tenant values, and also the importance of not restricting oneself to a predefined path for workshops based on one's own preconceptions, as sessions will undoubtedly will throw up unexpected obstacles.

Secondly, it is now clear that a critical component of participatory work missing from my process at Te Ara Hou (especially these workshops) was the need for participants to 'buy into' the work, and want to take part as they can see tangible 
benefits in it for themselves and/or their community. Participants of my workshops took part because they were interested and had time on their hands (at best) or because their mother told them to (at worst). This resulted in a general disengagement from the process. General apathy is always an inevitable hurdle in public engagement work, however it was only further compounded in my case due to the hypothetical nature of what I was doing. This reflection starkly contrasts with equivalent sessions at the Kenya workshop, which were always well attended and full of engaged, vocal (even bordering on aggressive, in some cases) participants. Residents could see their engagement could influence a future upgrade of their settlement, and consequently would have a dramatic effect on their livelihoods.

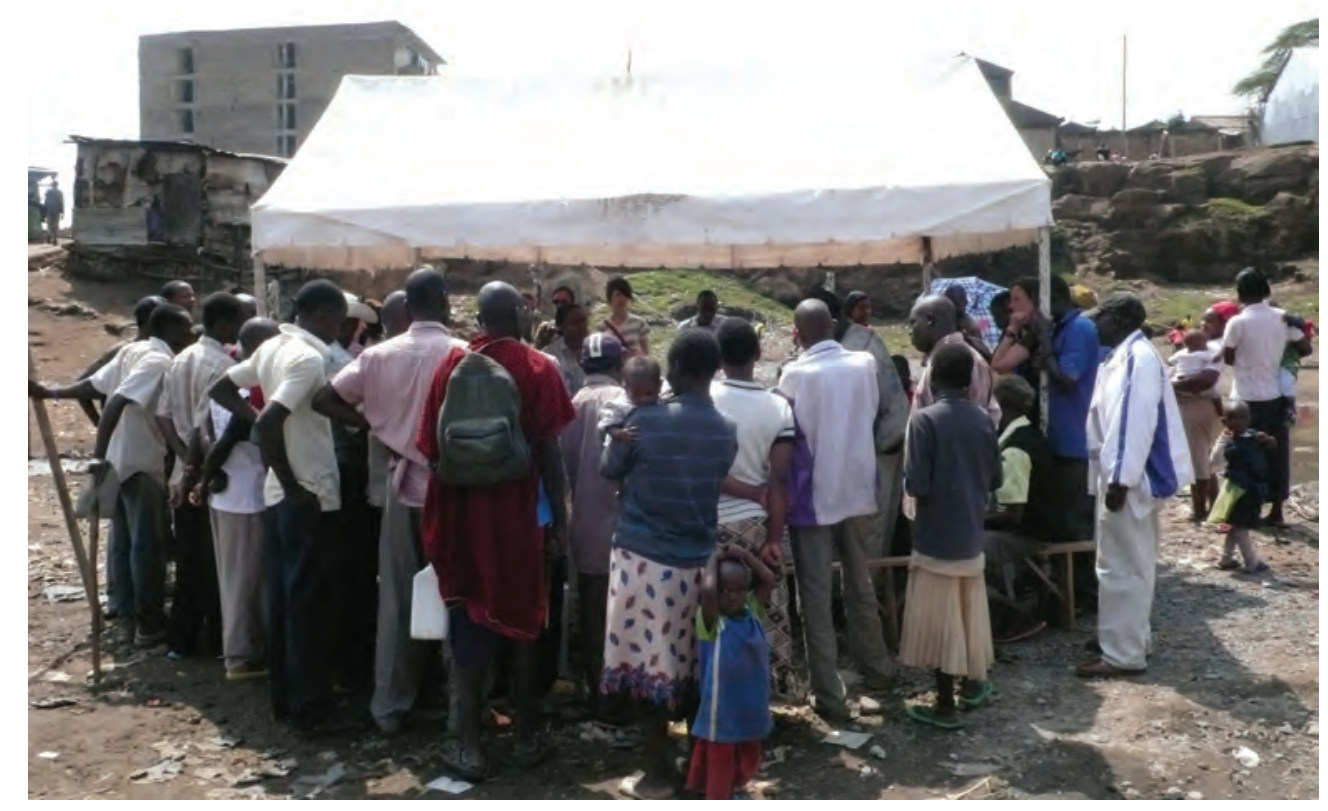

Figure 5.21 - A wellsubscribed workshop in Kenya - we had buy-in.

It is difficult to imagine how I could have designed the process for my project to overcome this hurdle, short of undertaking the work at a complex that was yet to be upgraded (as I had originally intended with Arlington Apartments - see 5.1.1). The main weakness of these workshops, therefore, stemmed from the WCC-imposed change of site from a yet-to-be-upgraded site (Arlington) to an already-upgraded site (Te Ara Hou). If I could have assured participants that their involvement was going to directly influence their upcoming upgrade (i.e. integrate my research work into WCC's actual engagement programme for that complex), it is likely participants would have been more motivated and engaged - more akin to the Kenyan sessions. Another possible way to help overcome this hurdle could have been to offer some sort of 'artificial' incentive (e.g. a voucher) to encourage residents to get involved in my workshops. One must be cautious with such incentives, however, as they can alter participants reasons for taking part which can have a negative effect on the work. It seems an ideal participatory programme is one in which 
people want to take part as the benefits of the work are tangible for them (i.e. the incentives for taking part are genuine, not artificial). Perhaps offering incentives in return for participation is more appropriate for more passive participatory approaches, like offering an attractive prize for those who return a survey. Another factor in obtaining buy-in for the work is how involved participants have been in earlier stages of the process. Some continuity is important to ensure participants are prepared and motivated for subsequent steps and understand how a particular step falls within a broader process. That said, it is important for a process not to be seen to becoming closed to an exclusive group of people who have been involved from the beginning, and remains open to accept new participants at later stages. In any case, these reflections do confirm the point that - whether in Nairobi or Wellington - participant buy-in is a critical component of a successful participatory programme.

To conclude, my three workshops seemed ineffective in delivering a proposal with the kind of rigorous development of spatial ideas I was expecting, and for that reason I was initially disappointed with their outcomes. In hindsight, however, I can see that my workshops were most effective in enlightening me to two fundamentals of participatory process work. Firstly, it is important not to set the path of a workshop too rigidly based on potentially incorrect assumptions and, secondly, participant buy-in to the work is critical. Chambers' sailing metaphor - that "good workshops are ... like a sea voyage" requiring responsive steering and tacking with the wind (2002:xiv) - offers insight based on these two findings. Looking back, it is clear that I was attempting to ignore the direction of the wind and motor forward defiantly toward what I had predefined as a desirable destination and, more broadly, it could be said that my work was simply lacking wind in the first place!

\section{INTERVIEW TECHNIQUE}

Over the course of my 17 interviews, I learnt the value of playing a more passive role in dialogue in order to fully reveal a respondents' unique point of view, and not drown it out with my own suggestions or findings from previous respondents. Zeisel eloquently describes this art as "[negotiating] with a respondent to find correspondence between [the interviewer's] own analytic structure and the respondent's mental picture of the situation" (2006:228). I learnt the value of spending more time discussing the respondent's perspective in dialogue, and only prompting with my own 'analytic structure' when it was required to keep a conversation 
flowing or if a respondent was having trouble understanding a 'question.' Taking the position of a 'potential convert' to a respondent's ideas is another suggestion of Zeisel's I have found to be useful in drawing information from respondents (2006:223). In all, I found interviewing an enjoyable, subtle art and my proficiency at conducting a good semi-structured interview developed over the course of my field work.

\title{
INEVITABLE BIAS AND RISK OF CO-OPTATION-
}

\author{
64 New housing would be great - great idea! \\ They could put it up over these car parks ... it \\ could be new housing for people to buy - be- \\ cause people here get really dependant and \\ need something to move on into."
}

Participant I

By pure coincidence, this participant's suggestion was exactly what I had in mind. Earlier in the project working at Arlington Apartments I had planned to workshop on an infill housing scheme tenants could buy into, which had the strategic agenda of getting people out of subsidised council housing and back onto the private property ladder. My reaction to this participants' comment was excitement, as I could now confidently say that the idea came from a tenant, not myself. It was like I'd been given my ticket and I could run off and design a scheme back in studio.

Whether the idea is right or wrong is irrelevant (I do still think it has merit) - what I should have been conscious of, however, was the way I was looking for reinforcement of my own ideas. Looking back, my excitement should have been very closely followed with concern that I might be fatally ignoring one of participatory design's best practice fundamentals - that one must never, through the guise of a participatory process, co-opt participants' contributions and use them in pursuit of one's own agenda (Cooke 2004, Cooke and Kothari 2001). From this realisation, I proceeded with the idea of new housing on the site with more caution and selfreflection.

Achieving a position of pure neutrality or objectivity as a facilitator is impossible - one will always carry their interests and agendas. What is critical, however, is keeping track of these, and never manipulating or co-opting participants contribu- 
tions in pursuit of these interests and agendas.

I finished the two month field work phase with a mass of information from participants; snippets of ideas, suggestions to improve the place, both encouragement and discouragement to further some of the ideas for interventions I had tested through the process, and a good general sense of the diverse range of views within the complex. Most importantly, I had confirmed that participatory techniques beyond those WCC are already engaging showed potential to be beneficial to future processes. On the whole, strategies influenced by ASF's Kenya workshop translated into a Wellington context well. The remainder of this chapter continues the documentation of this 'design experiment' as some of these many threads are pulled together into a proposal. 


\subsection{Building understanding of elements of 'a brief'}

\subsubsection{Introduction}

This sub-chapter carries findings from the participatory exercises forward into a brief for an intervention on the site, and builds understanding around two elements of that brief (WCC community rooms and medium density housing precedents).

This sub-chapter, together with the following sub-chapter 'presentation of a physical intervention' (5.4), explore the edge of participatory process and are, in a way, more akin to a traditional top-down 'professional-centric' design process. They engage skills more familiar to an architect like precedent analysis, design of building forms and architectural representation. It must be reiterated, however, that these sub-chapters are still in pursuit of the overall objective of this project; to develop a better participatory approach. The exploration of a physical intervention is simply another means through which completed participatory exercises can be tested and considered. These sub-chapters address the question, 'how do bottom-up design processes interact with more top-down, professional-centric design processes?' This question is addressed at the conclusion of 5.4.

Following the establishment of a brief (5.3.2), case study research is conducted on medium density housing precedents (5.3.3) and WCC community spaces (5.3.4). The method for conducting these case studies was guided by the 'research methodology' sub-chapter (1.4). They were discrete pieces of research in that they did not involve participation with tenants.

\subsubsection{Creating a brief}

The following two-part brief emerged to direct the remainder of this 'design experiment.' Most aspects of this brief originate from the participatory exercises outlined in 5.2 - a sentence in italics follows each briefing point to explain the origins of that element. 


\section{NEW HOUSING UNITS}

Although the idea of intensification of the site was partly unpopular, it is progressed with here as it did generate some support from participants, and this exercise could be of wider use in the Housing Upgrade Programme as many sites across the portfolio include excess land that represents significant commercial development opportunity.

- Propose units is the North-east corner of the complex between the car parking area and Constable St. 'Discussions over drawings' revealed that of the three potential sites (see Figure 5.14), this was the preferred site for intensification.

- Retain all existing car parks (add more if possible). All three participatory exercises revealed car parking to be of great concern to tenants.

- Retain existing Pohutukawa trees where possible. Two separate interviews revealed these trees to be important to tenants.

- Design units to be relatively low-cost housing. Occupants could either be WCC tenants or private residents following their sale. I deemed it beyond the scope of this project to determine who could occupy units. Interviews revealed both support and opposition to either scenario.

\section{B. COMMUNITY SPACE}

This is proposed in direct response to a finding from the field work, that a group of tenants want to

\section{Briefing points:}

- Re-instate the disused laundry room in the South-East corner of the site as a community space for a range of tenants to use. Firstly interviews, then also workshops and 'discussions over drawings' revealed this as a desired outcome for tenants.

- Consider uses like homework groups, a casual playgroup for mothers, display of certificates and trophies celebrating achievements of youth within the community, access to computers/the internet. These ideas have emerged from tenants via interviews.

- Ensure facility is inclusive of a range of social groups and age groups. Discussion with WCC revealed that a previous community space on the site (now the crèche) was discontinued by WCC as it became controlled by one closed group of tenants.

- Consider the very limited budget of $\$ 5,000-\$ 10,000$ for the fit out. Discussion with WCC revealed they have a plan to develop this room, so I wanted to keep the proposal in reach of what might actually happen at the site. 


\subsubsection{Case study research - Medium density housing precedents}

I needed to look to high quality precedents of medium density housing projects in order to design of units at Te Ara Hou with more confidence. I wanted to compare some of the findings from my participatory exercises with examples of high quality medium density housing. The first criterion for selecting case studies in this inquiry into high quality medium-density housing is the New Zealand Institute of Architects (NZIA) architecture award programme. As the most comprehensive architecture awards programme in the country with an online archive of 10 years of winning projects, it was deemed to be an appropriate setting in which I could find the best quality housing projects in the country. The next criterion was to find projects within the archive that were similar in scale and characteristics to what could be proposed at Te Ara Hou.

Two extra case studies fell outside of this first criterion, but were still deemed particularly relevant examples as they aligned closely with the typology proposed at Te Ara Hou in different ways.

In total, six projects are presented individually in Appendix D. Details on each project include photographs, the NZIA award the project won and 'lessons for the Te Ara Hou infill development', which includes my own analysis and any other relevant comments from sources like the award citation, websites of architects, blogs, or other books. This section now draws findings from the case study analysis and discusses them in relation to findings from the participatory exercises.

\section{OVERALL THEMES}

It is evident from the case study analysis that the provision of seclusion and privacy in medium density environments is key to a successful development. This has been achieved repeatedly though the precedents with deep balconies/decks which create more privacy for interior spaces (Trinity Apartments, Thackeray St), and is also evident as much attention is paid in the design of outdoor space immediately outside ground floor units (Beaumont Quarter, Trinity Apartments, St Mary's Court). The garden outside the ground floor apartment (see Figure D14, Appendix D) achieves a high level of seclusion as all views into the space are blocked. In a less extreme example, Beaven achieves a degree of privacy in the ground floor outdoor spaces of St Mary's Court by blocking views from neighbouring outdoor spaces (with a block wall), but allowing views out to the communal 
space in front of the unit through a grille fence. Another strategy to create more private living spaces is to lift these up to the first floor (Gore St). This finding from the case studies directly reinforces tenant values revealed through my participatory exercises. Tenants repeatedly made it clear that they liked the semi-private outdoor spaces for ground floor units, and this had vastly improved the complex since its upgrade. It became clear that new housing on the site should follow this lead. Whether an NZIA jury or a Te Ara Hou tenant, all seem to agree (in different language) that private outdoor space is desirable.

The cases studied present a range of strategies with regards to the provision of spaces for the public. Kerstin Thompson is clear that architects of medium density housing developments must consider how to best serve the public realm through the design of medium density housing developments (Murray 2008:22). Beaumont Quarter offers public lawn spaces (see Figure D3), although they are likely to be used more by residents than passers-by as they are located internally within the site plan (see Figure D1). Beaven observes that the lawns of St Mary's Court aren't particularly well-used by residents as they prefer their own more private spaces. My participatory exercises similarly revealed much consideration of interaction of public with sites. The playground on the Te Ara Hou site is very well-liked by tenants and, on the whole, tenants were more than happy for members of the public to be using the playground (there is an interesting parallel here with a finding from the ASF workshop where Jacqueline dreamed of providing a playground on her property for public use - see Box 1 in 4.3.3). They seemed proud that people came to enjoy 'their facilities.' That said, there was some concern expressed around public thoroughfare through the site. Most are OK with people passing through, but some did not appreciate people being able to look into their bedrooms from a public path. As the site selected for new housing at Te Ara Hou offers an opportunity to provide a strip of public space along the busy footpath of Constable Street, attention will be paid to the design of that space as it seems that a contribution to the public realm is an important component of quality medium density housing.

In developments that incorporate existing buildings, a strong theme in these successful examples is the acknowledgement and reference to these existing buildings (particularly evident in St Mary's Court, also the Ebor Street Townhouses and Beaumont Quarter). This is done through a linking material palette, and relating building forms (heights, roof pitches, etc) of new buildings to those of old. On this theme of acknowledging the history of sites, the retention of existing trees amongst new buildings at Beaumont Quarter is noted in its citation. Although my 
participatory exercises did not reveal any findings on relating to existing buildings, some tenants did express attachment to the existing Pohutukawa trees on the patch of grass in the North-East corner of the site. Similarly, while workshopping on ideal upgraded settlements through the 'Portfolio of Options' exercise in Kenya (see 4.3.5), participants were unanimously keen to retain the existing main street through the settlement in any upgrade plans. Although we all pay attention to different aspects (trees, a street or a material palette or roof pitch), most seem to agree that it is important to acknowledge and retain aspects of our existing environments as we dream about new ones.

Three other findings from the housing case study research offer insights for a proposal, although were not found to relate to findings from my participatory exercises:

- St Mary's Court offers good entry strategy as, even in a relatively small one bedroom apartment, the entry is separate from the door connecting interior and exterior living spaces. This adds to the seclusion of the outdoor space as it is not passed through to approach the entry door.

- One characteristic setting medium density housing apart from higher density apartment buildings is that individual units are more easily distinguishable. The Ebor St Townhouses offer a good example of how to express each unit separately, as party walls make the distinction clear (see Figure D18).

- Finally, Gore St Housing by Kerstin Thompson Architects is a good example of designing a building that meets the needs of today whilst remaining flexible to accommodate adaptive re-use into the future (see floor plans, Figure D17). I will endeavour to follow this philosophy wherever possible at Te Ara Hou.

\subsubsection{Case study research - WCC community spaces}

Relating to 'the other half' of the proposal, the community space, I visited working examples of WCC's community spaces in other complexes. This was to learn from what is already working (or not working) in these spaces, and to see how existing spaces related to what Te Ara Hou tenants were wanting developed at their site. To assist me, WCC arranged a tour of some examples of these community spaces with a staff member from within the 'Community Action' team at City Housing, who was familiar with the implementation and management of these spaces. 
Community spaces are included in 14 of WCC's housing complexes and vary greatly in their scale and complexity. Some are simple rooms, others include a full range of facilities. The initial suggestion of a space can come from either tenants or staff within the Community Action team (within WCC). They are implemented by the Community Action team who work closely with a complex to identify needs particular to them that could be supported by a space. In some cases, this consultation extends to workshops with tenants. WCC then scope possible areas within complexes that could be developed, and implement plans as budgets allow. Sometimes budget restrictions mean work is completed in several phases within an overall 'masterplan.' A challenge for WCC is to balance tenants ideas for community spaces against their commitment to the ratepayer to be economical in their delivery of housing services.

Management of the facilities is done both centrally (by the Housing team at WCC), and also locally by tenants who are appointed by WCC as 'Community Room Coordinators' (CRCs). The larger, more complex facilities are managed more centrally while the smaller facilities can be managed more locally. Generally, CRCs are particularly engaged tenants who are known well to WCC staff.

We were limited in the number of cases we could visit due to time constraints so, as is good practice in case study research, we opted to view a selection of spaces that were extreme cases so I got an overall sense of the variety of solutions (Huberman and Miles 2002:12). The case studies range from the elaborate 630 square meter purpose-designed 'complex' of facilities at Central Park to a modest 32 square meter space in an old laundry room at Pukehinau Apartments.

Each of the four spaces we visited is profiled individually in Appendix E. Details include locality plans, approximate floor plans, photographs and written points about each space. This sub-chapter now draws findings from the case study analysis and discusses them in relation to findings from the participatory exercises.

\section{OVERALL THEMES}

The most pressing consideration after visiting the four spaces was the importance of fostering inclusion with these spaces, ensuring they cater for a diverse range of interests and activities. It is clear that the facility within Central Park will achieve this more easily given its sheer size and range of spaces. Whare Ahuru's facility - being in a converted flat - benefits from having four small separated spaces, 
so that a range of activities can take place simultaneously. For example, having a dedicated room for sewing means that other activities can happen at the same time and also that machines can remain set up. The level of finishes in each room can then suit a range of activities, too, for instance the art studio can be a bare concrete garage, whilst a meeting space is carpeted and more comfortable. The facilities with only one shared space (Pukehinau, Hansen) are limited in that they don't support 'installed' activities (like sewing and art) as easily, although perhaps this issue of cross programming is something that the ensuing physical proposal at Te Ara Hou can explore. Discussions around a community space at Te Ara Hou through the participatory exercises certainly did reveal a wide range of potentially conflicting uses. Some wanted to see computers provided, others want it to be a space for creating art, while others see it as a good space for running a playgroup in. Analysis of existing case studies did not reveal any particularly innovative solutions to cross-programming although, as mentioned above, perhaps this is something the physical intervention sub-chapter (5.3) can explore.

At one of the case studies, a potential issue is developing as one tenant has claimed the use of a space to themselves as their art studio. With these more locally-managed facilities, this matter of which tenants hold the keys for shared facilities has potential to become problematic as 'gate-keepers' could assert more control over the spaces as is appropriate. It is difficult to know how the design of a facility could mitigate this potential, beyond perhaps mentioning that a swipe access system integrated with tenants access to their units could be more diplomatic system of entry than keys for community spaces. This is more of a management issue, largely beyond the scope of this study.

'Architectural quality,' in terms of natural light and ventilation, was lacking at the Whare Ahuru space. This is acceptable in the circumstances, and a community space (only occupied some of the time) is certainly a better use of a low-quality basement apartment than an actual unit for tenants. That said, it is worth mentioning that achieving architectural quality in community spaces within complexes will be a common challenge as they are often allocated to 'left-over' spaces like basement apartments and disused laundries. Participatory exercises at Te Ara Hou certainly revealed that tenants wanted the quality of the space to be improved as much as possible, as tenants know its current low-grade condition as their ex-laundry room. If community spaces are important elements of complexes then wherever possible they should be purpose-designed into complexes (like at Central Park), rather than provided as after thoughts. This would help ensure resulting 
spaces are of as high architectural quality as possible.

It is clear across all facilities - large or small - that a kitchen in a necessary component to prepare hot drinks, to wash art equipment, to prepare/store food, etc. Also across all four examples, it was evident that privacy for a tenancy manager to conduct their regular clinics with tenants was important (clinics are fixed times approximately once a fortnight where tenants can visit their manager on site to discuss concerns). The ideal is to have a separate office with its own toilet and kitchenette. Interestingly tenants at Te Ara Hou did not explicitly discuss a need for a kitchen in their space through the participatory exercises, which reveals to me that it is not their first priority for the space.

Analysis of case studies showed that consideration of communal needs for outdoor events/activities is important in designing a community space, too. The green plastic chairs in the Pukehinau space (see Figure E11) were requested by tenants so that they could easily relocate them outside for BBQs. A storage area for the $\mathrm{BBQ}$ on the ground floor is another important component of Pukehinau's 'community space.' The servicing of these outdoor needs must also be considered in the design of a community space although, as with the provision of a kitchen, this was not the most important aspect revealed through my participatory exercises.

Finally, Hansen Court offers a good precedent for how a community facility can 'tie a complex together.' Although it is too early to say whether the facility is having that effect socially, as it is not yet being used, it is certainly physically wellpositioned to do so being centrally located between each of the three parts of the complex. The architects of its upgrade, Architecture + , have representing this unity rather explicitly in their colour choices for the space; each 'highlight' colour used in the three parts of the complex is brought together in the colour choices of the community space. Concentrating other outdoor activities like vegetable gardening near the facility is also positive as it presents a clear 'heart' to the complex. Hansen's facility is a good example of how, through good siting and the central concentration of a range of activities, a community space can go beyond simply providing space for people to do things in; it has the potential to become the framework around which the social heart of a complex can develop. The potential for this has become evident at Te Ara Hou, too, as many tenants have mentioned how well the communal gardening area works. A new community space offers an opportunity to consider other aspects like this gardening area. 


\subsubsection{Other reflections for the brief}

Moving forward towards a community space proposal for Te Ara Hou, it is worth noting the comparatively limited scope of proposed work that might go ahead at Te Ara Hou. The most modest case study from the tour - the space at Pukehinau - was converted from a laundry room in 2010 at a cost of around $\$ 50,000$, and the speculatively proposed budget for a space at Te Ara Hou is around $\$ 5,000$ $\$ 10,000$. Although it would not be appropriate for this study to get too involved in costing the proposed works, in does give a broad idea of what might be plausible to go ahead.

Two parting thoughts the Community Action staff member left me with at the end of our tour were that, firstly, there are already strong gardening activities at Te Ara Hou - could that be built upon? Also, it would be wrong to reinvent what's already happening nearby in other facilities, like existing play groups in Newtown Hall and an existing computer hub one block away on Constable Street, so be mindful of that.

In all, these two pieces of case study research (on medium density housing and WCC community spaces) provided an interesting comparison with what I had learnt through the participatory exercises. They proved that somewhat 'non-participatory' exercises like these can still add to findings from a participatory programme. They have complimented the findings from my participatory exercises well, reinforcing some aspects and prompting further questions/exploration of other aspects.

A physical intervention is now presented, which has explored how a more traditional top-down design process can slot into a participatory-based approach. 


\subsection{Presentation of a physical intervention}

\subsubsection{Introduction}

This sub-chapter presents the physical intervention now proposed for the Te Ara Hou site. It explores how findings from earlier participatory exercises can be expressed through a proposal, and also how those findings can be complimented by a more top-down design process.

The result of this stage of the 'design experiment' is a relatively small-scale proposal. The community space component has been prepared with WCC's plan to carry out such a retrofit in mind, so it is modest to reflect their available budget.

The presentation style of the drawings that follow favours sketchy representations over photo-realistic imagery. This preference further reinforces the focus of thesis which is on an ongoing design process that doesn't result in a fixed, 'finished' design. The sketchy nature of drawings reinforce to those who would view them that the ideas put forward in the following proposed intervention remain open to further discussion and development, and are not a discrete end-state. 


\subsubsection{Drawings}

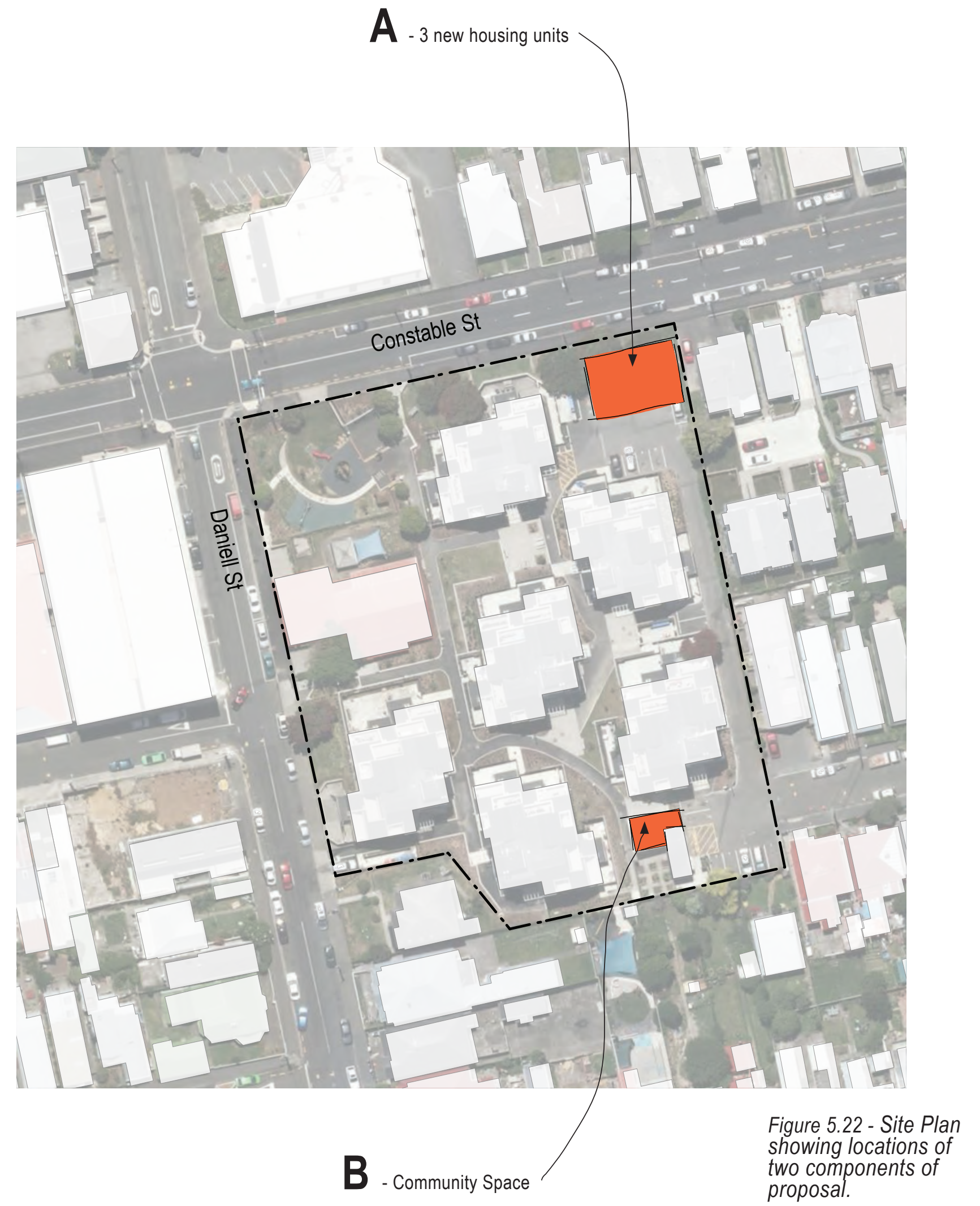

Scale 1:1000 


\section{A - 3 NEW HOUSING UNITS - EXISTING SITE CONDITIONS}

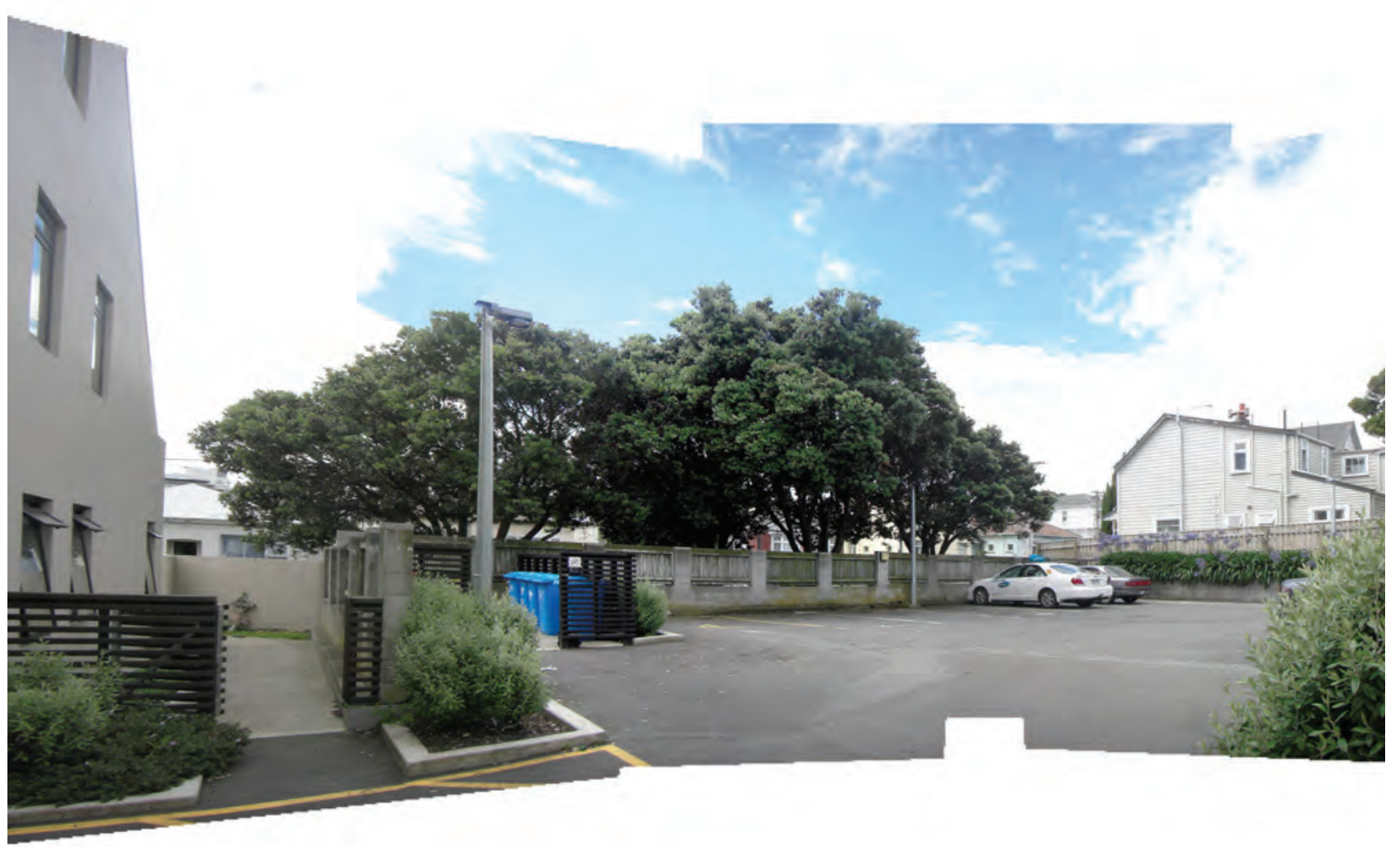

Figure 5.23 - Photo-

graph from inside complex looking to

car parking area 


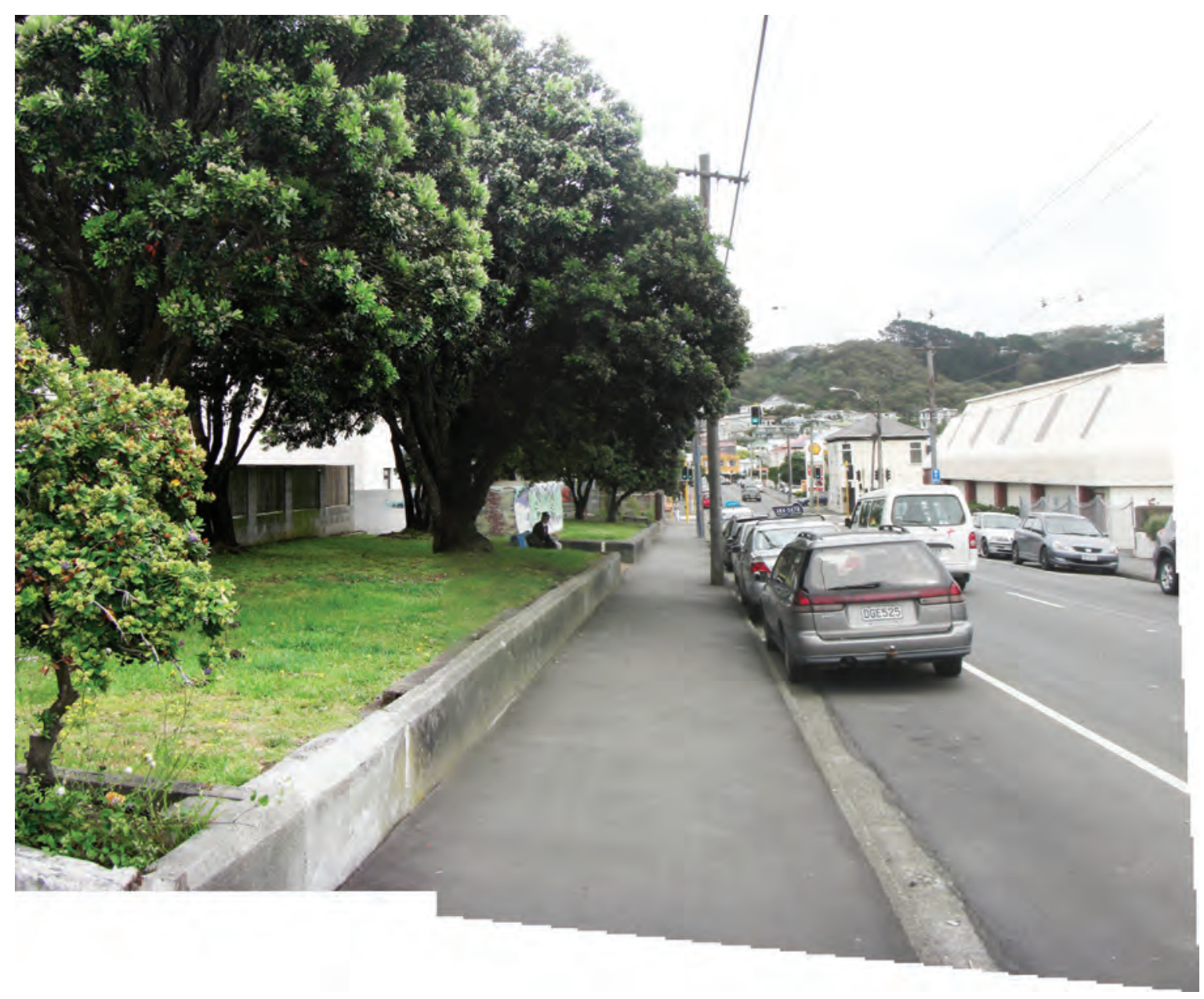

Figure 5.24 - Photograph looking westward along Constable Street 


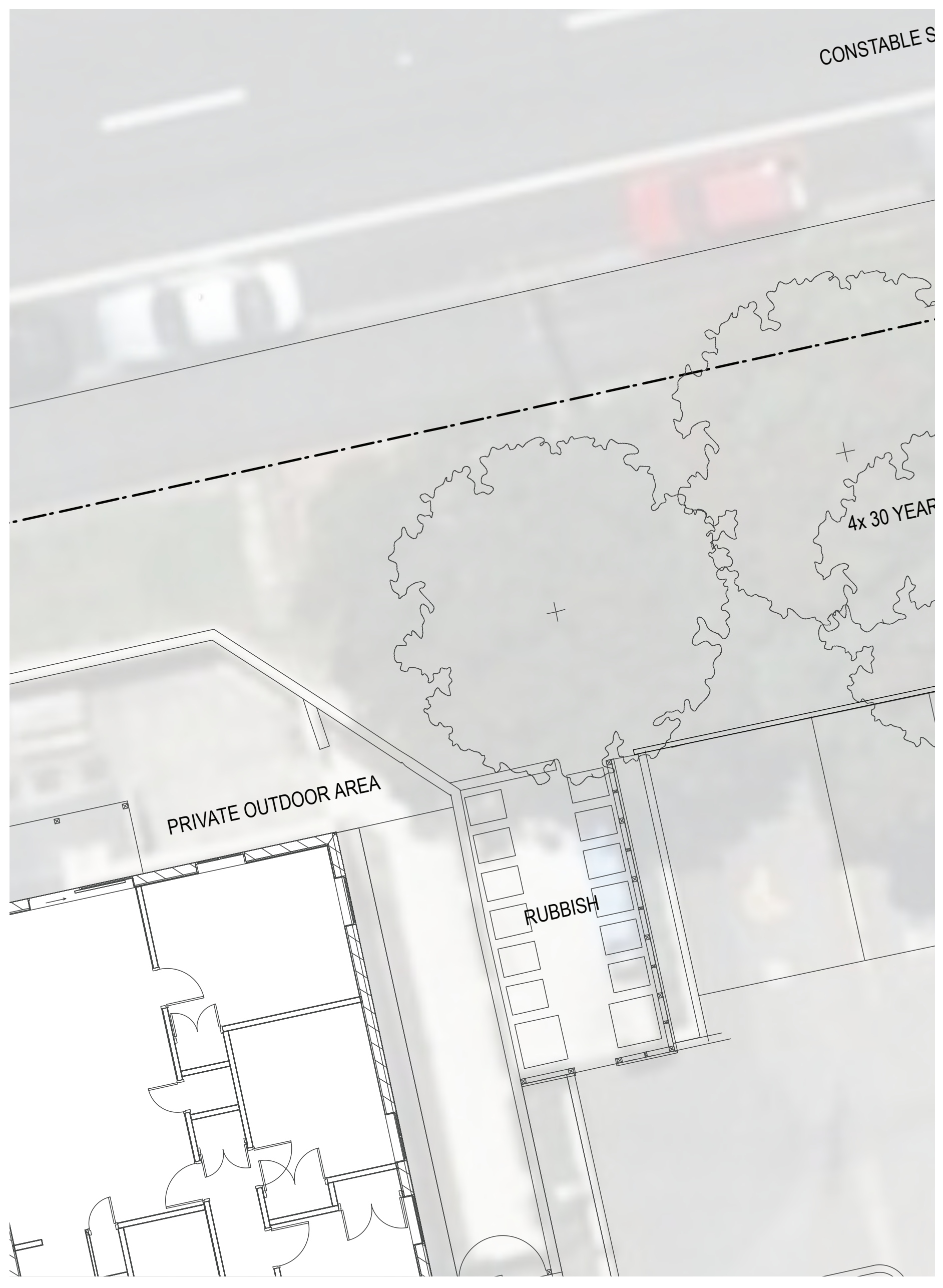




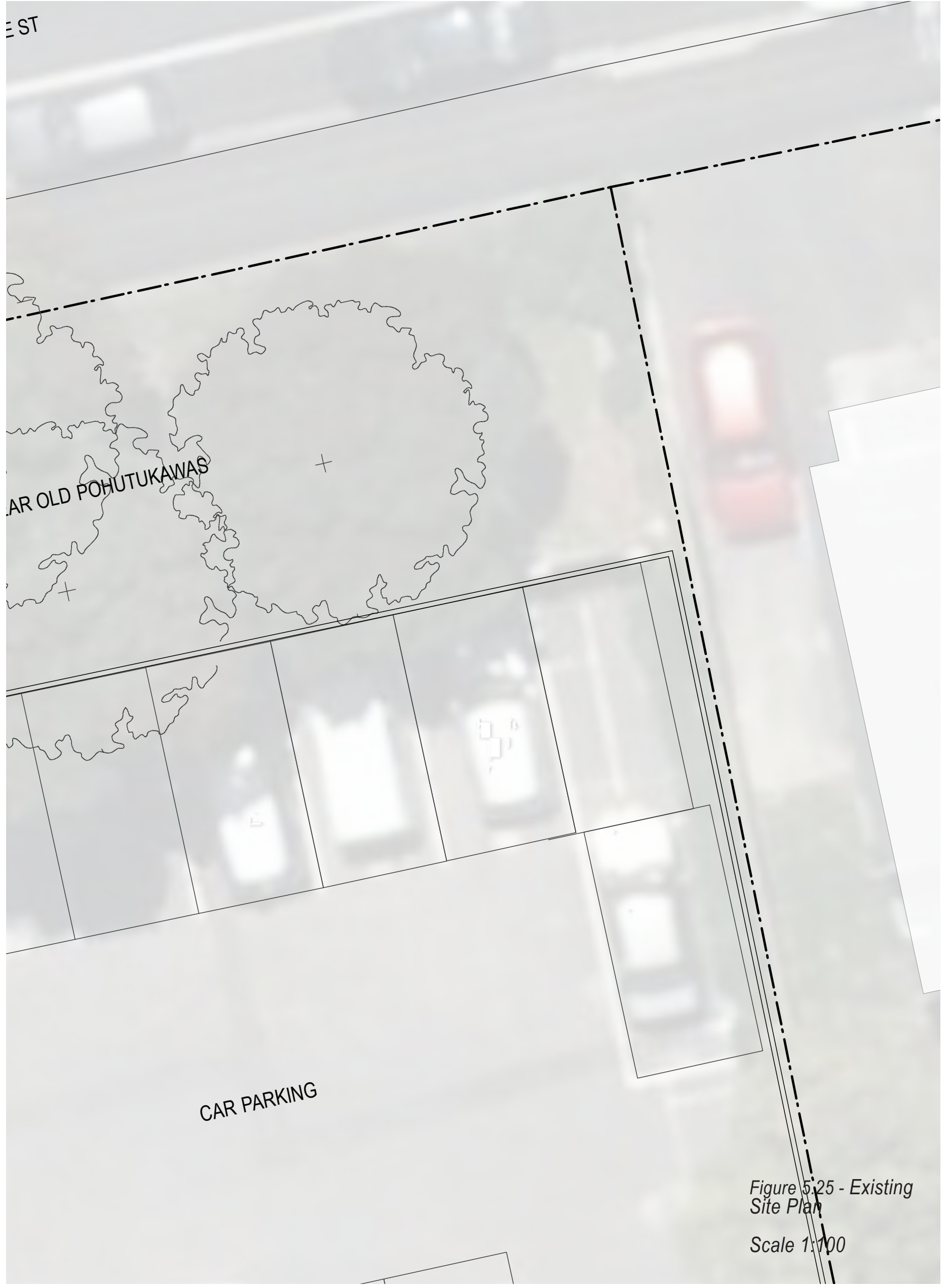




\section{A - 3 NEW HOUSING UNITS - PROPOSED BUILDING}

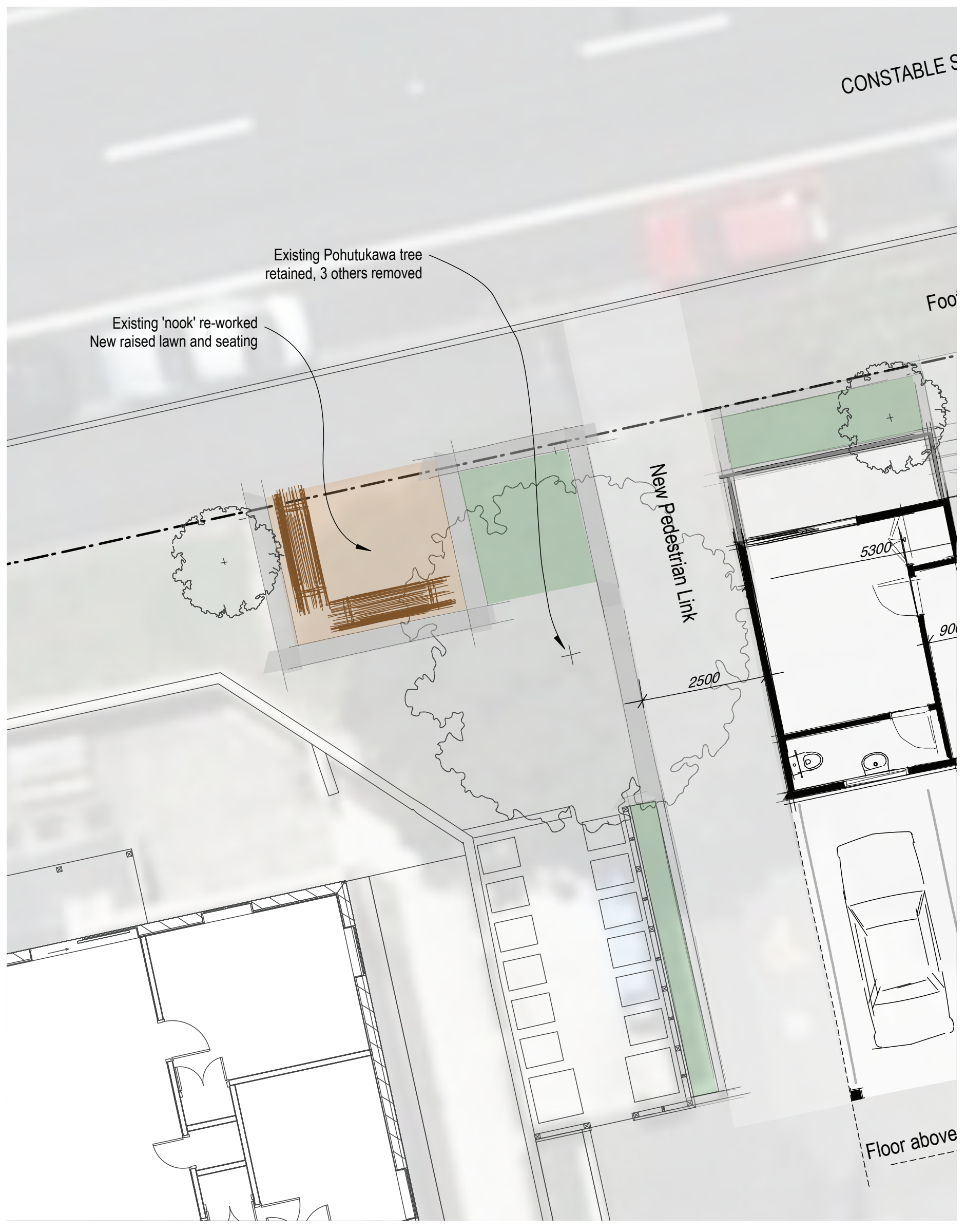




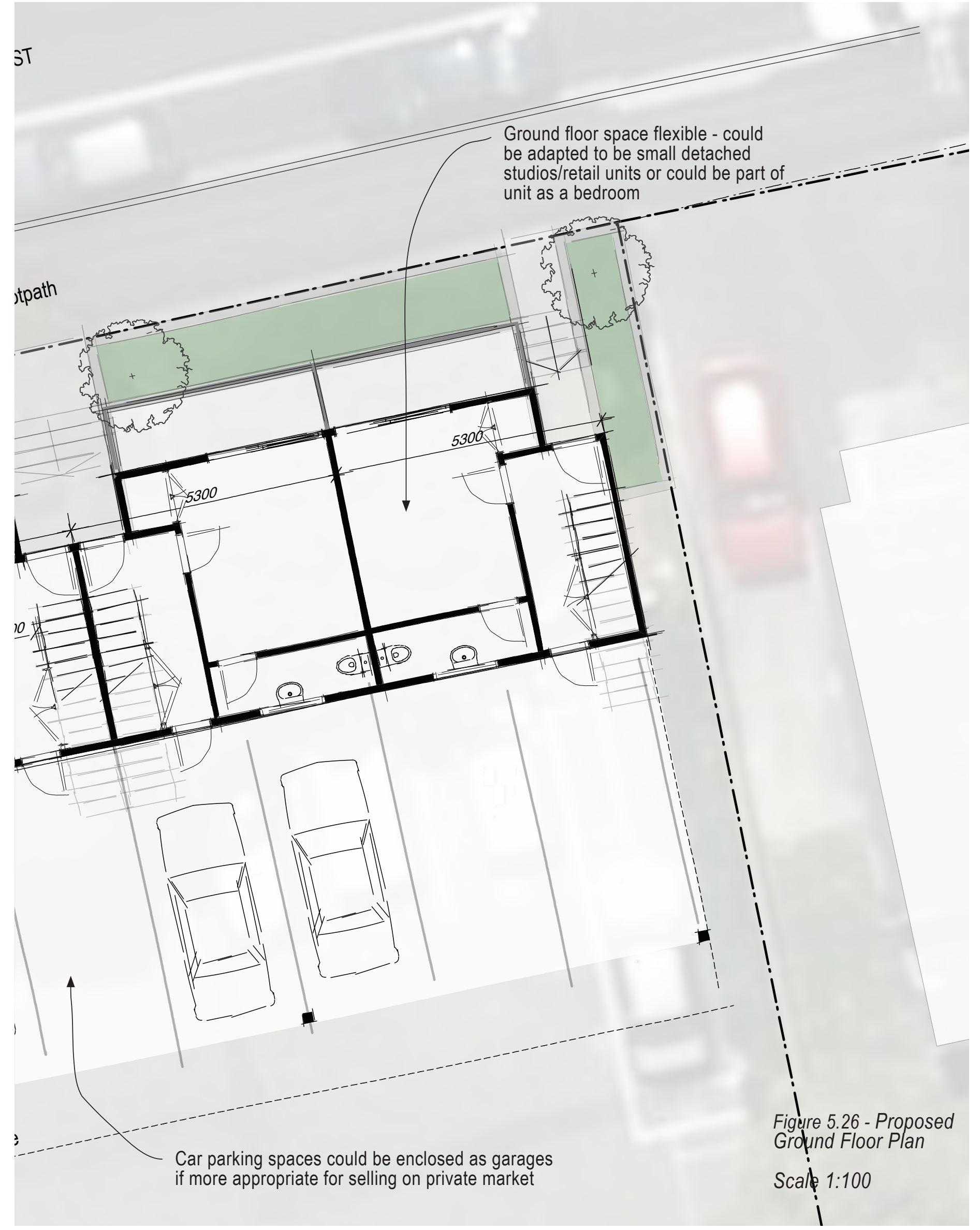





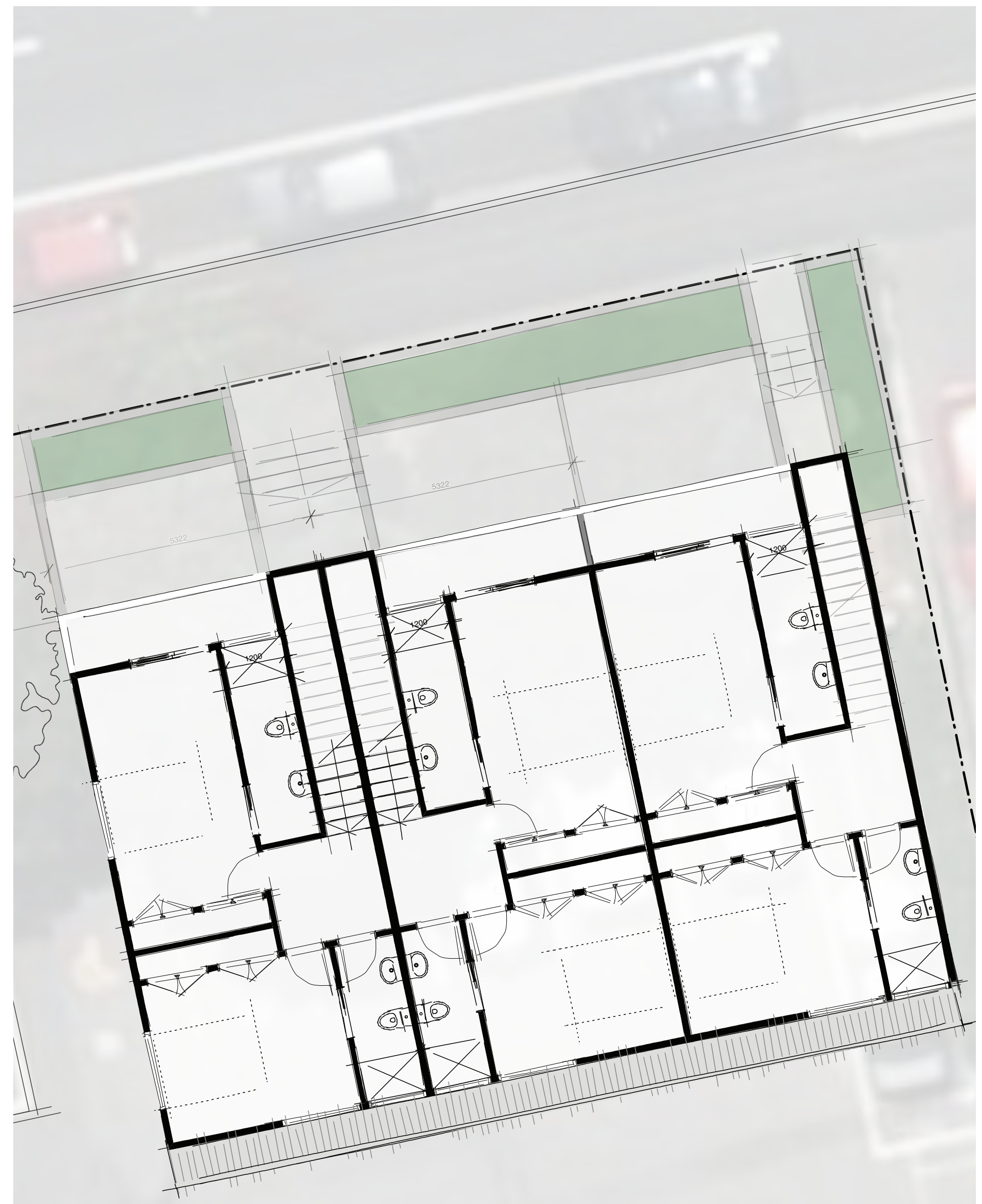

Figure 5.28 - Proposed Second Floor Plan

Scale 1:100 


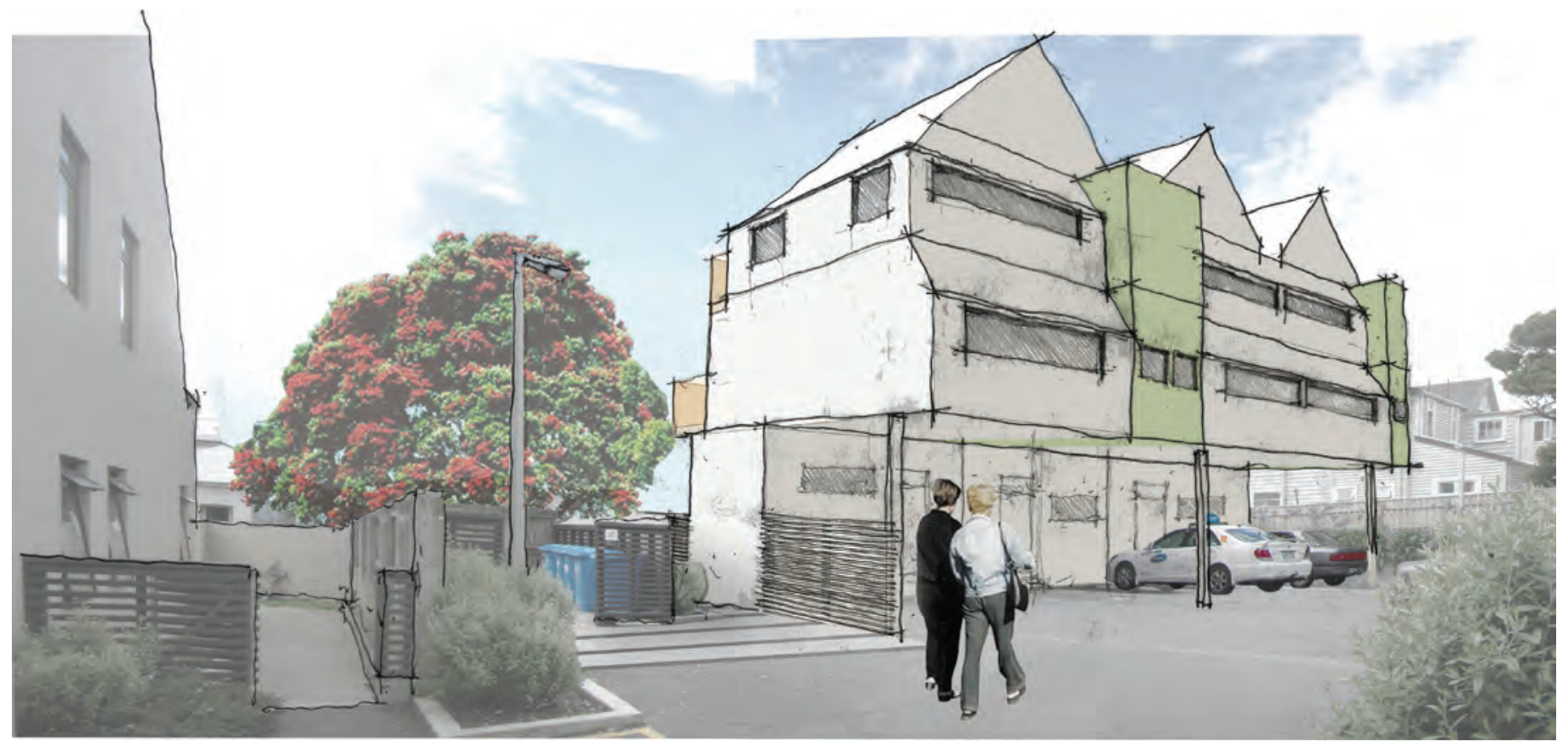

Figure 5.29 - Rear view 


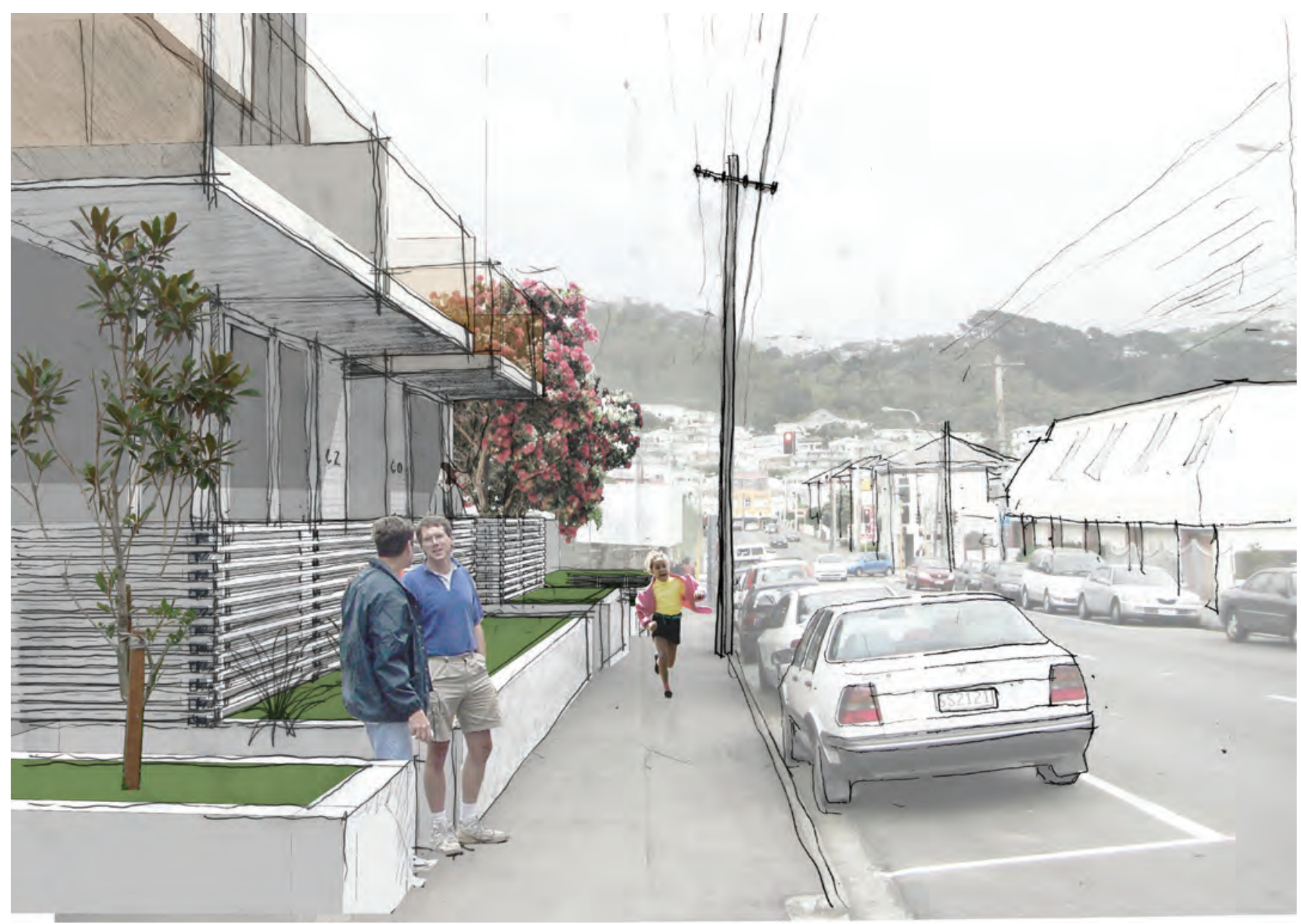

Figure 5.30 - View along Constable St 


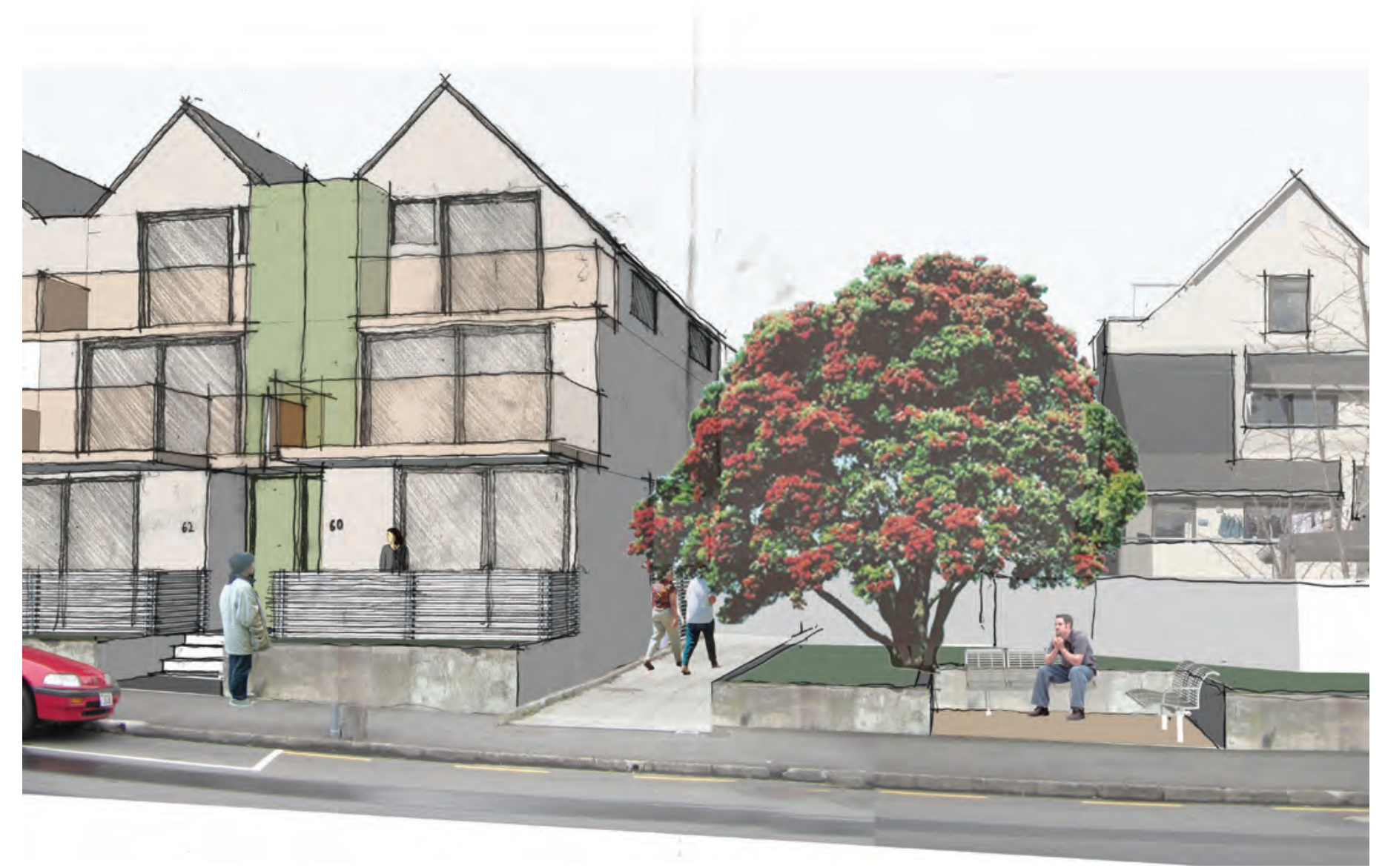

Figure 5.31 - View across Constable St 


\section{B - COMMUNITY SPACE - EXISTING SITE CONDITIONS}

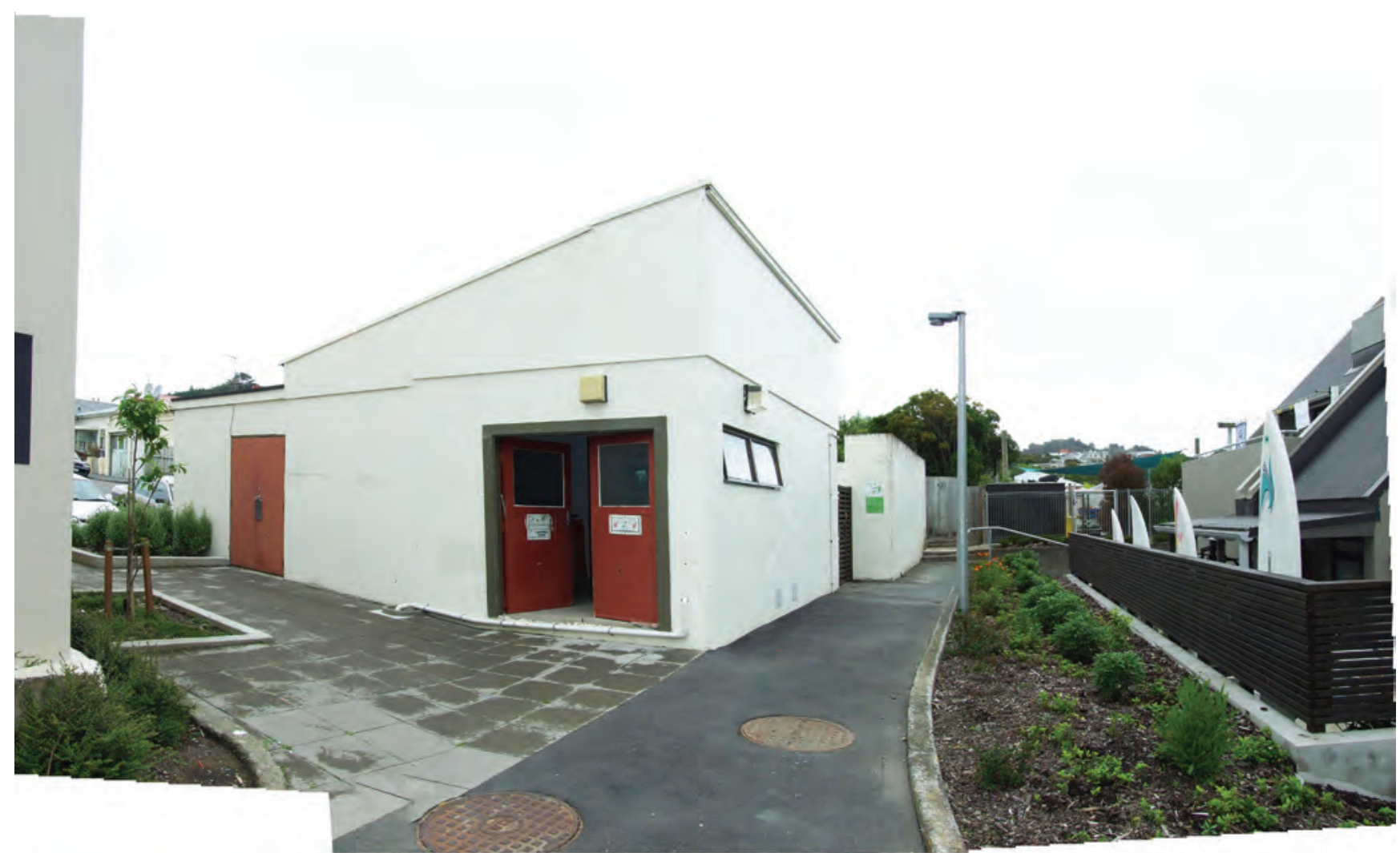

Figure 5.32 - Photo of exterior 


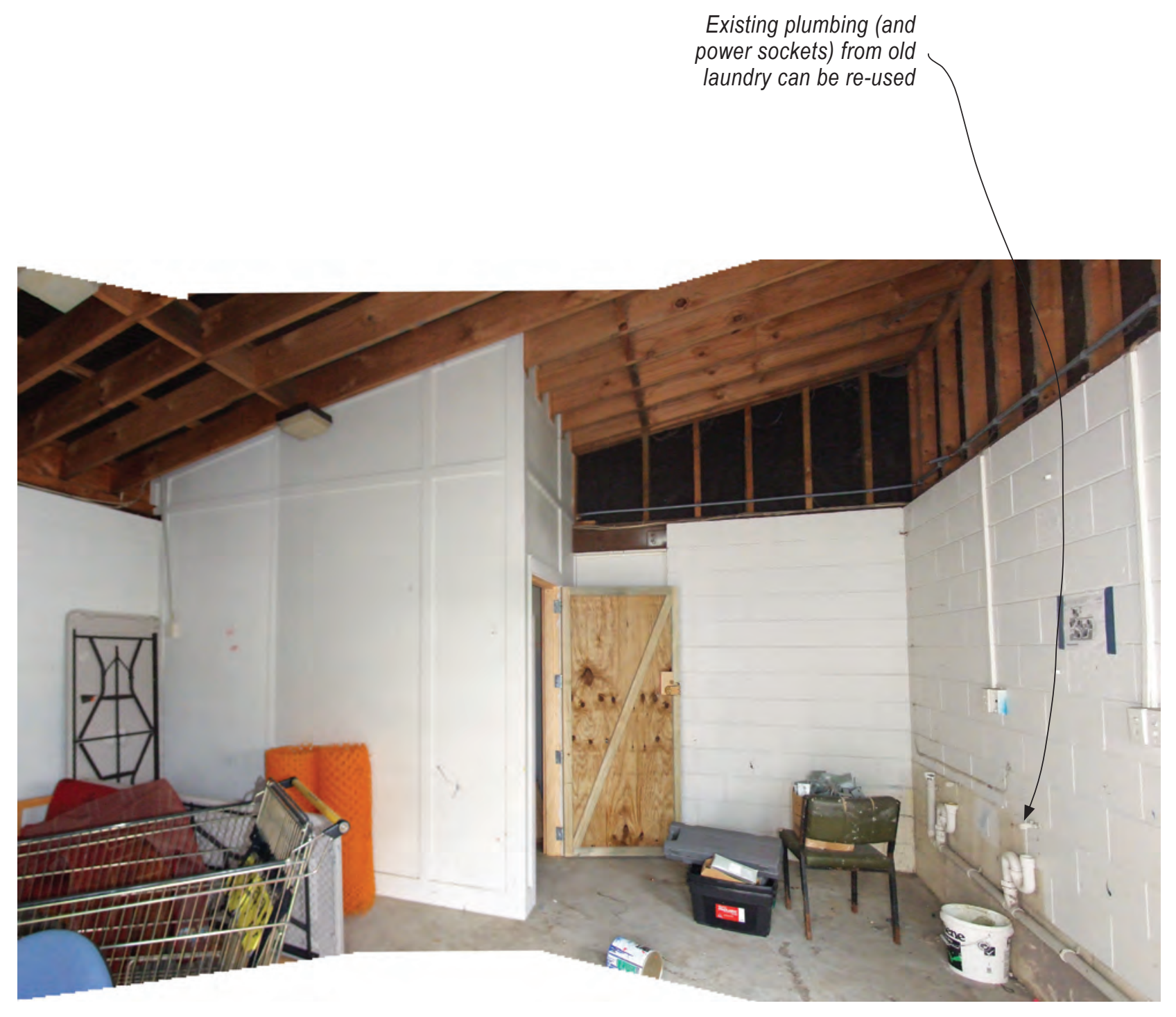

Figure 5.33 - Photograph of interior 

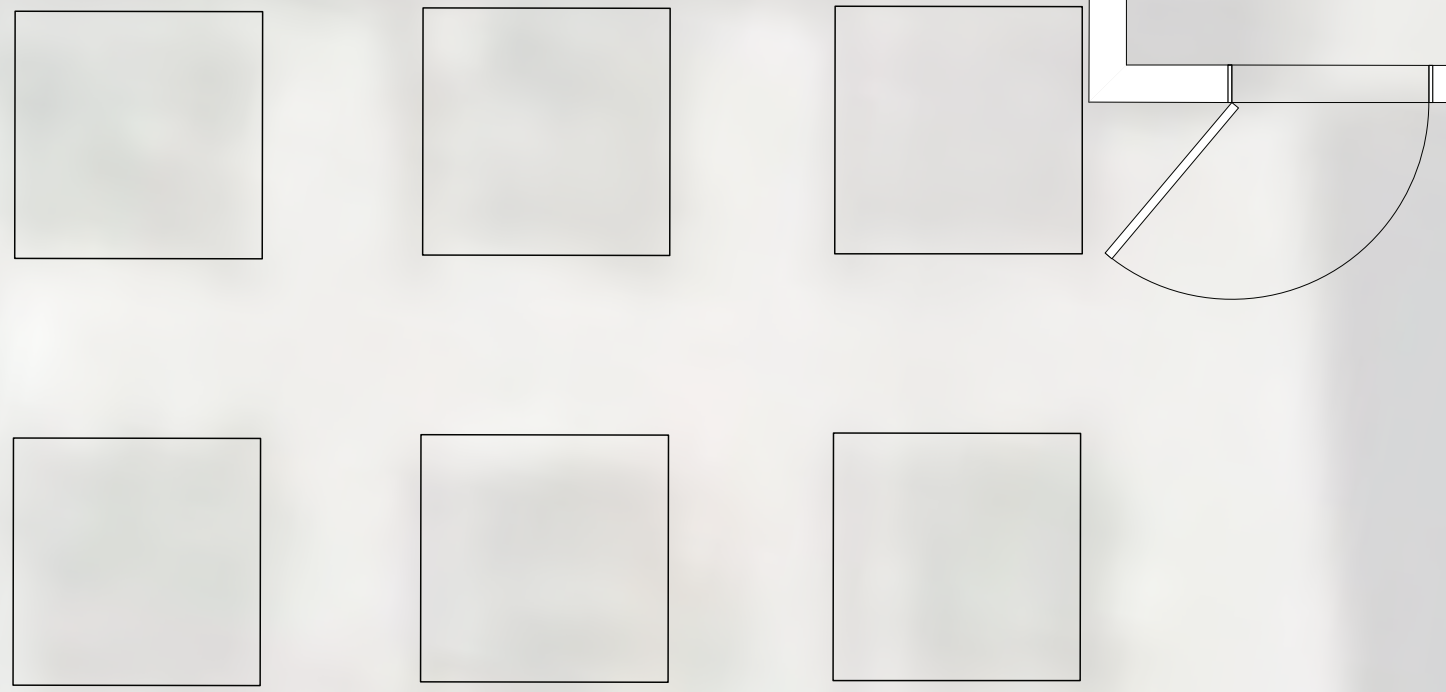

COMMUNAL GARDENING BOXES
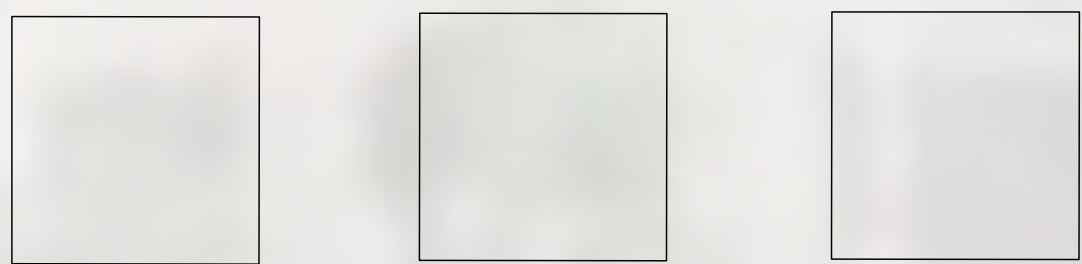

SWITCH ROOM

TRANSFORMER

RUBBISH AREA

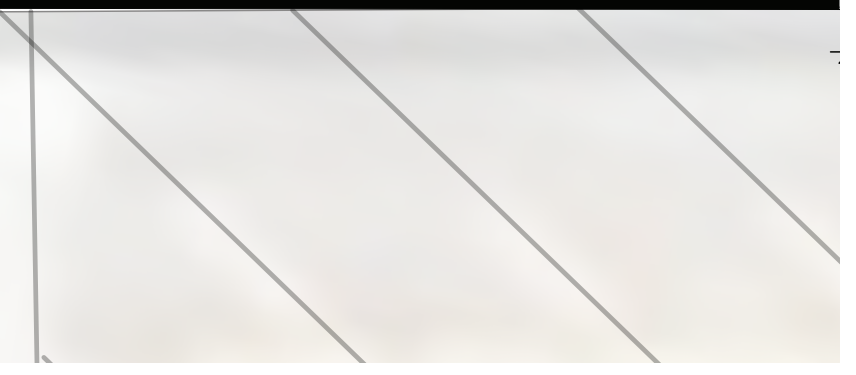



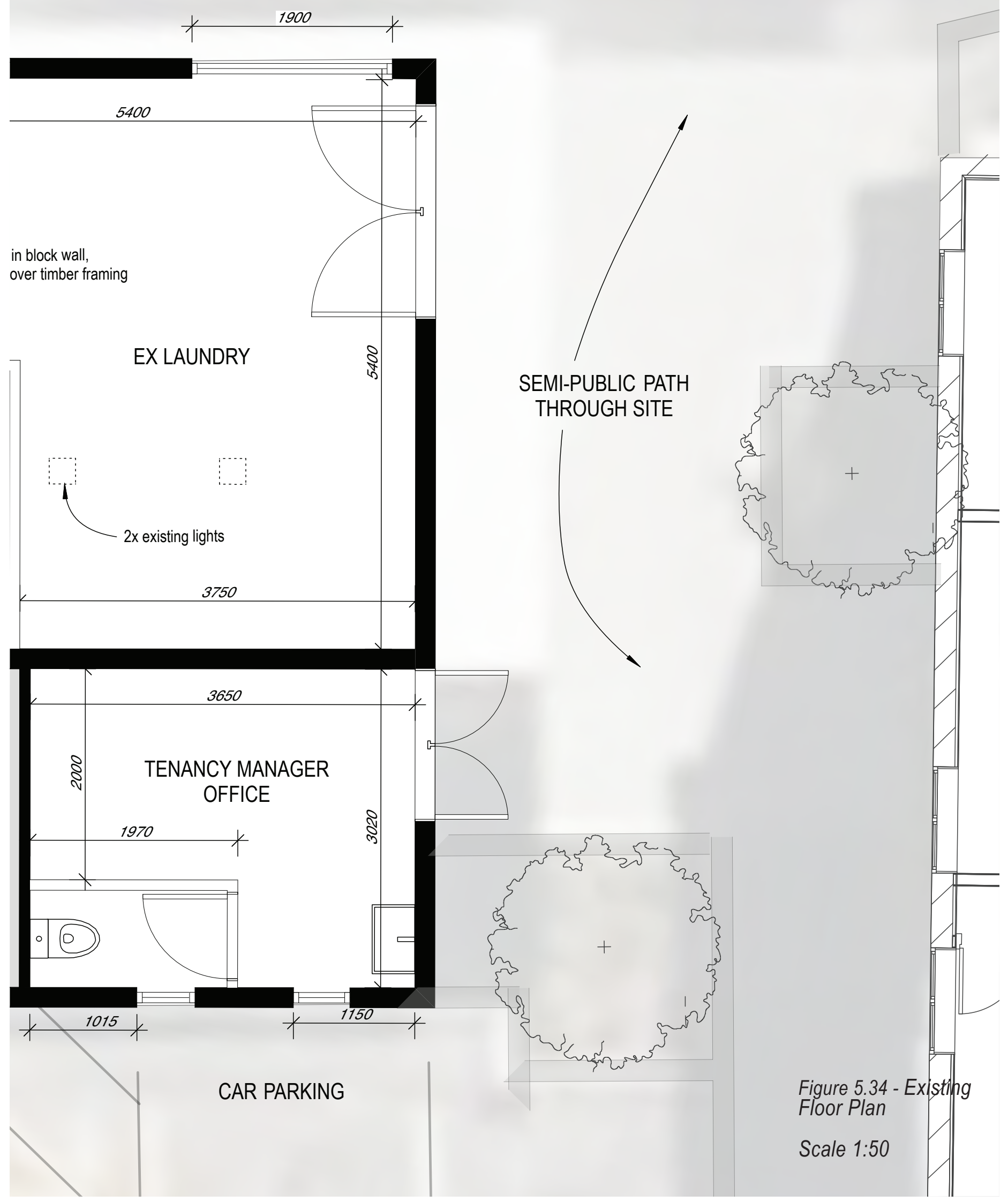


\section{B - COMMUNITY SPACE - PROPOSED INTERVENTION}

\section{Dooth}
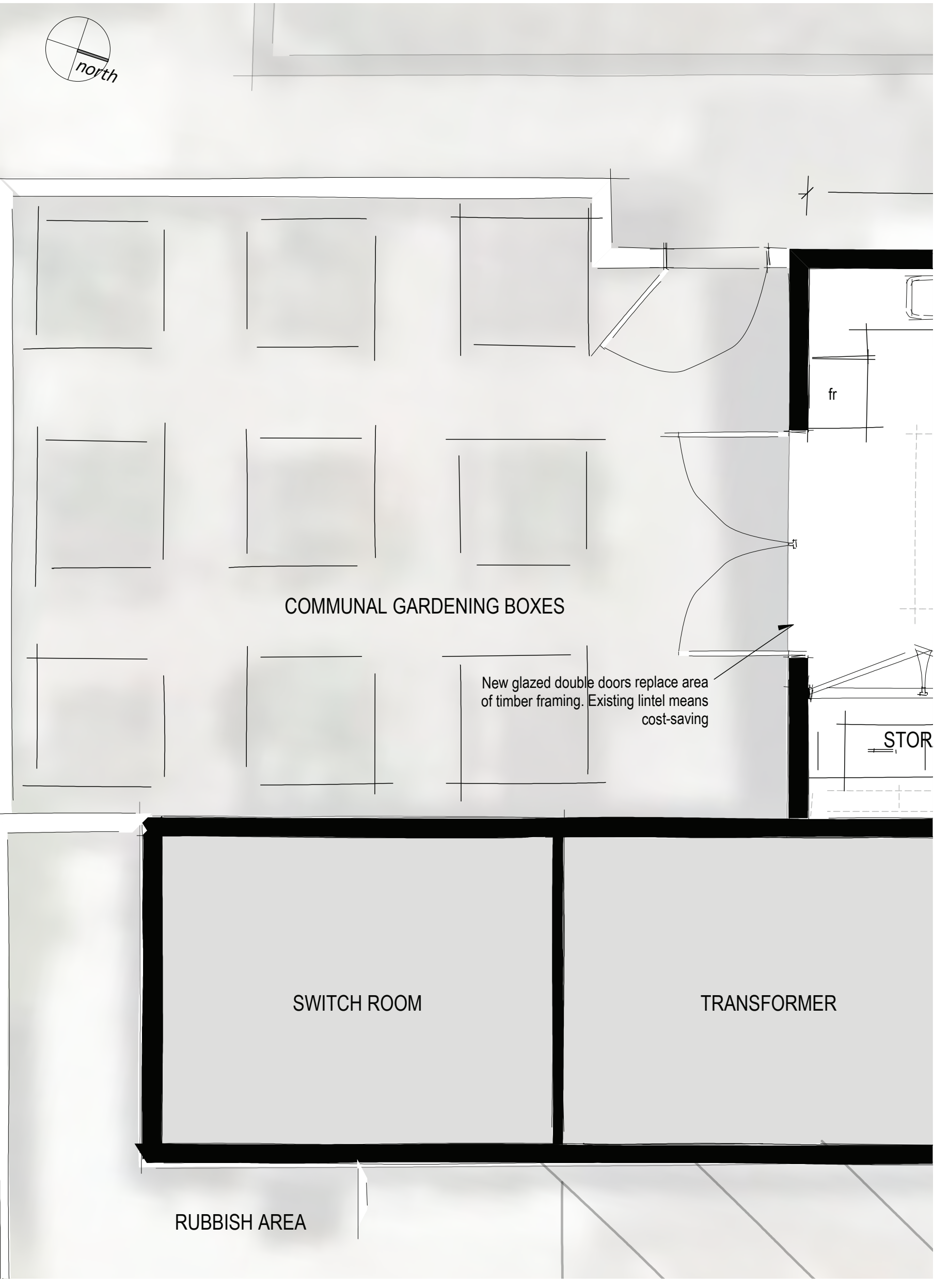


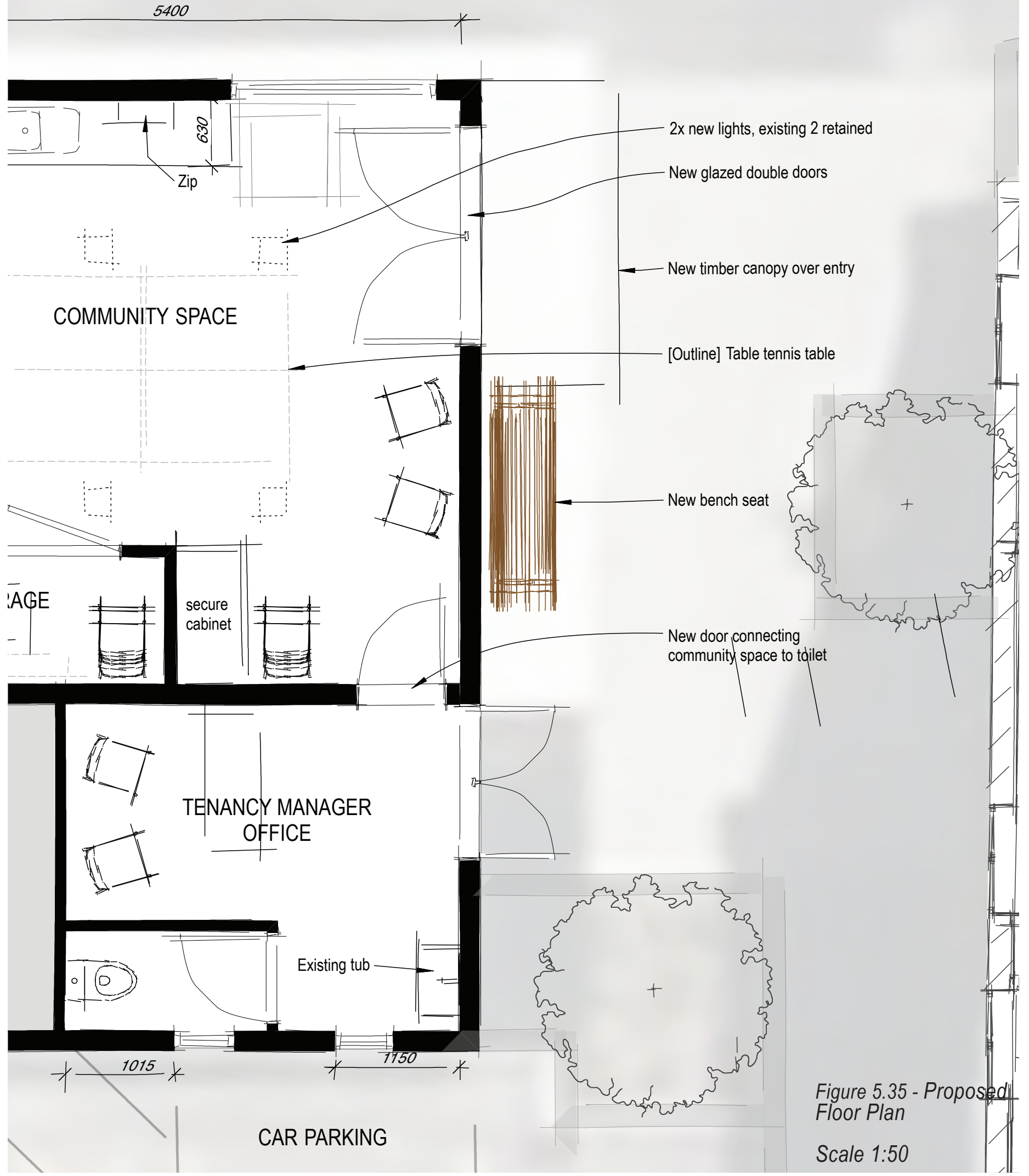


Wifi provided (inside and outside). Perhaps shared laptops locked in secure cupboard (keys with Community Room Coordi-

Large store fits $B B Q$, etc nators)

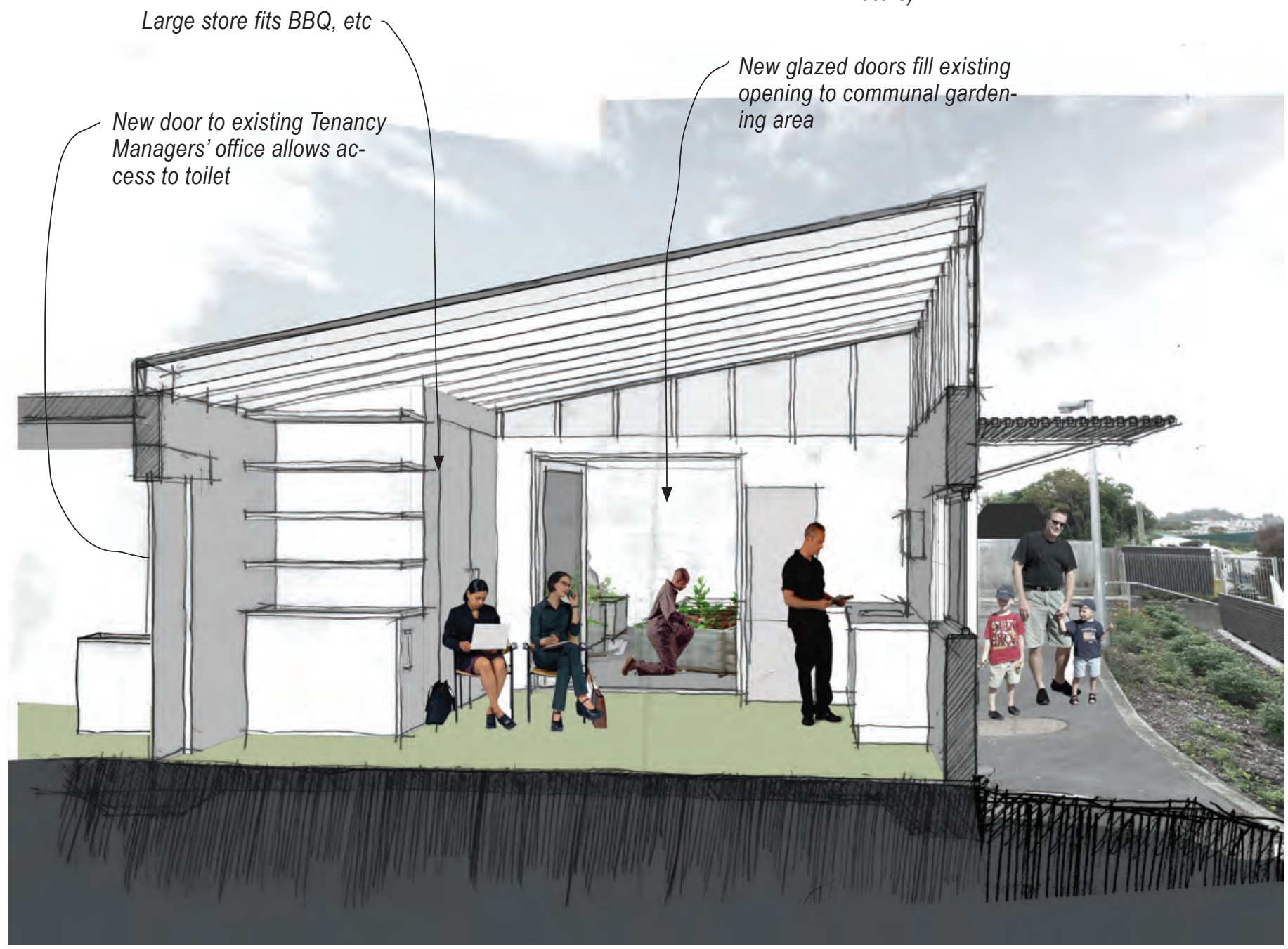

Figure 5.36 - Section through room 


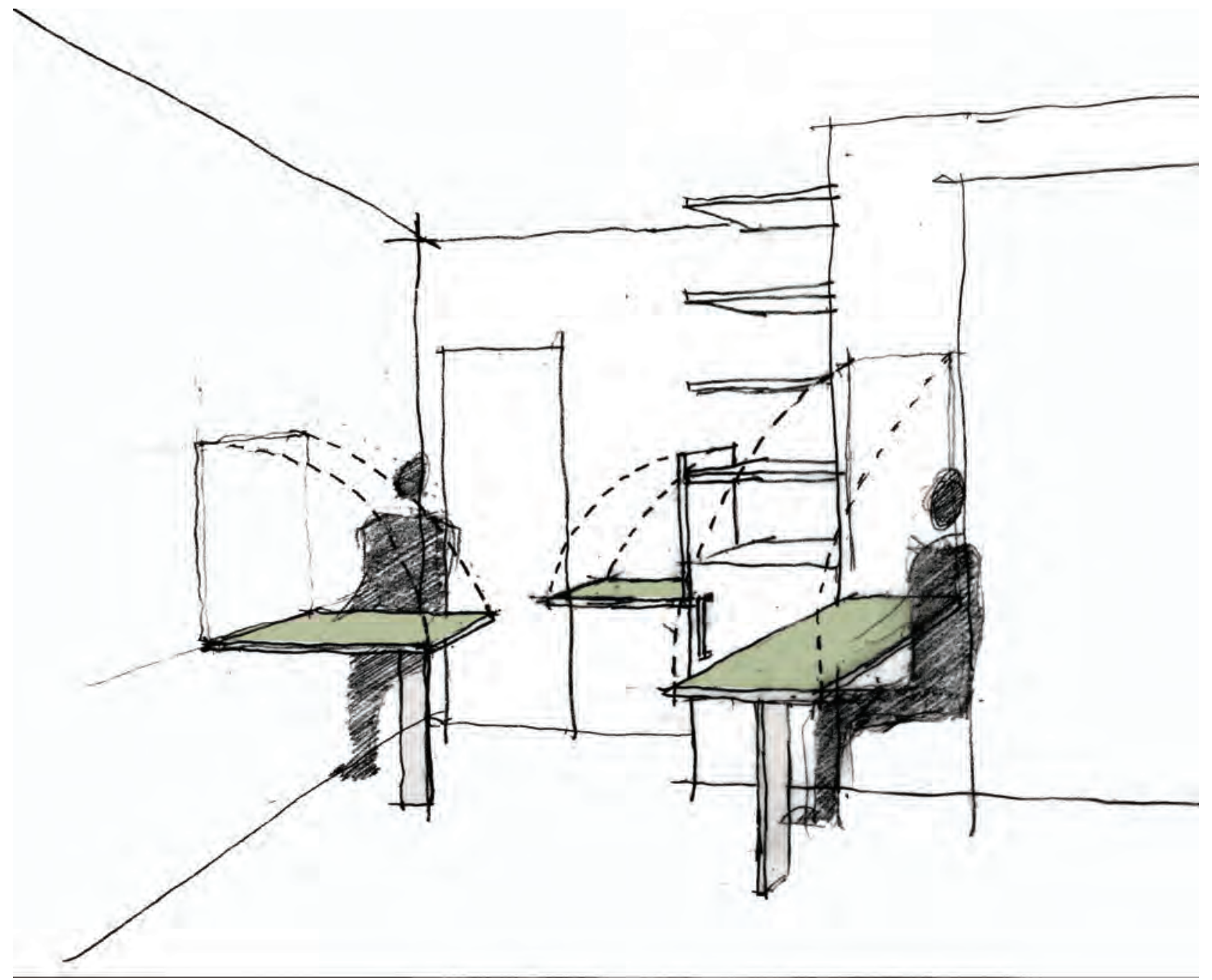

Figure 5.37 - Designing a cross-programmed small space: fold-down desks 


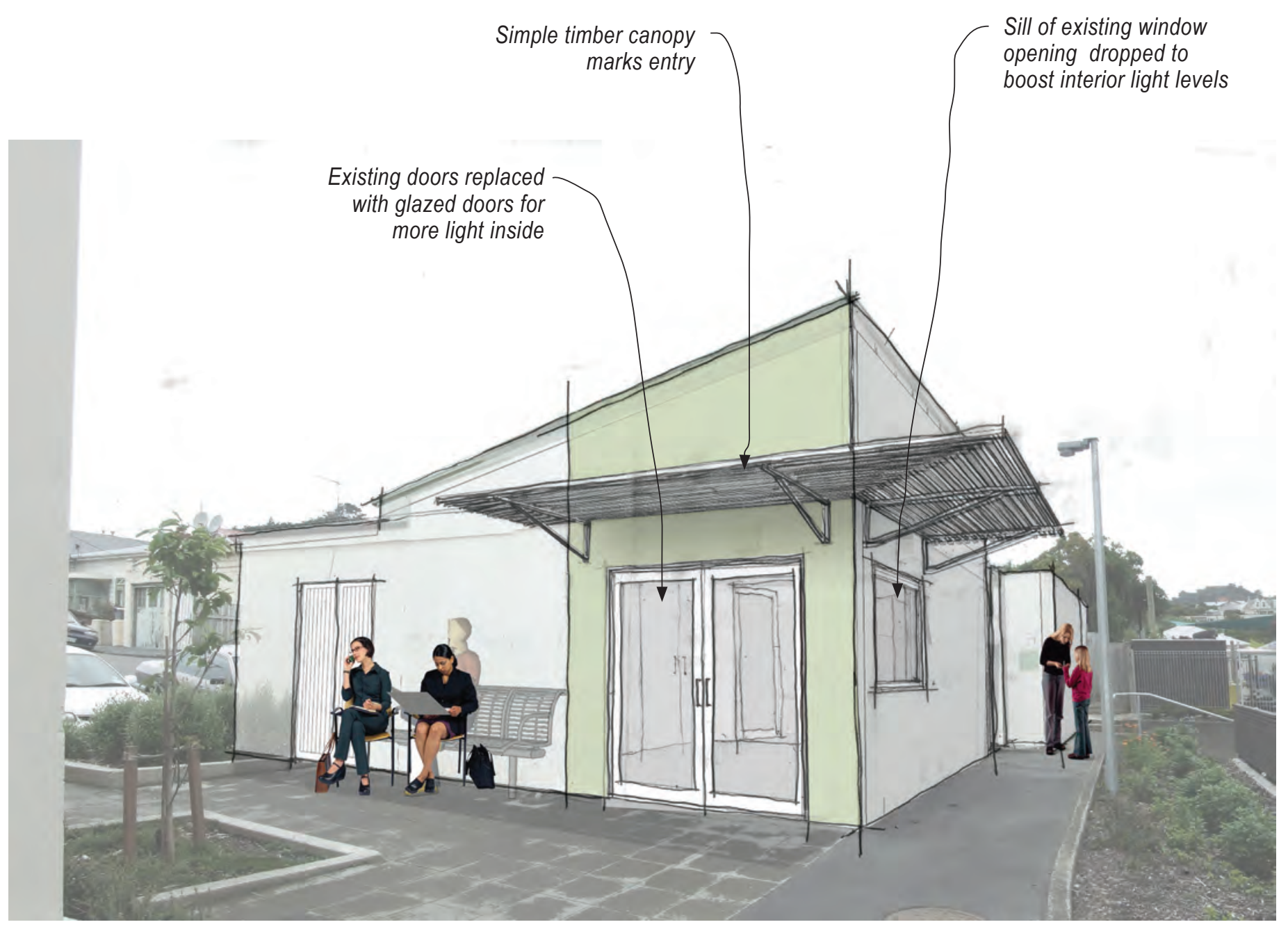

Figure 5.38 - View of exterior 


\subsubsection{Discussion}

The above proposal is now discussed in relation to previous steps along the 'design experiment'.

The above proposed intervention encapsulates many of the tenants' ideas that were revealed through earlier participatory exercises. The suggestion of folddown desks (see Figure 5.37) is one example of how a tenant's need for a space to run a small homework club has been developed into a solution that could fulfill that tenant's need. The tenant offered the idea and that was taken and developed. The case study analysis process, too, revealed unique insights. For example, in designing the building housing three new units greater attention was paid to referencing the existing built forms of Te Ara Hou following lessons learnt from the quality case studies. In all, 5.3 has revealed that even a process with a strong emphasis on participation should leave room for more conventional 'top-down' design process to occur as it too offers unique insights. Of course, it is critical that this more 'top down' process is intricately linked to surrounding participatory components and not an excuse to 'close the door' on participants and design in isolation.

As well as favouring the feasible over the implausible, I have explored a proposal that is more indeterminate than fixed in its proposed supported functions (particularly with regards to the community space). I have explored a design that "will not seek to designate a discrete 'end state' for the simple reason that there is none; ... the aim is to create conditions and not impose restrictions" (UN-Habitat 1987). Hamdi's notion of "an architecture of invitation and of opportunity" (2010:150) is also explored. This incremental approach seems appropriate in the circumstances of this community room as the budget is so limited. WCC could implement the proposal incrementally and respond to what is working and what isn't as they go. The above proposal should also be considered as being capable of being extended somtime in the future. Hamdi's point reinforces this approach:

The urgent need is to design strategies and institutions that can better integrate incomplete knowledge with experimental action into programmes of adaptive management and social learning." (2010:115)

Also in this spirit, the proposal of WiFi in the community space is significant. It is proposed that this could be a pilot project for WCC which, if successful, could be a catalyst for rolling out wireless internet hotspots through the portfolio. This was 
suggested by some tenants who said in many cases tenants do not have internet access in their units. The community space could become a hub for people wanting to use the internet with their own laptops, smartphones or to perhaps even use shared laptops that could be provided. ${ }^{3}$

Although the proposal does not go as far as suggesting who the new housing units are for (whether for City Housing tenants or sold on the private market), they have been informed by tenant preferences revealed through the field work. They are deliberately designed to read as separate 'Constable Street' units, rather than more 'Te Ara Hou' units. Some tenants engaged through the process expressed a preference for living on the edge of the complex, less communally. Others living in the heart of the complex liked the communal aspects. Of course, there will always be diverse preferences; these new units simply accommodate those wanting to live slightly more separate from the complex.

I now offer some reflection on how this top-down process of preparing a proposal (5.3 and 5.4) interfaces with the bottom-up participatory exercises of 5.2. In all, preparing this proposal (5.4) in a largely top-down manner did not jeopardise the integrity of findings from the earlier participatory exercises. The literature review taught me that participation should cultivate choice and flexibility in outcomes (2.3.6), and I believe the proposed intervention proves that some top-down design work can maintain that choice and flexibility, by designing a space for multiple uses and proposing a housing type that is somewhat unique from other Te Ara Hou units.

To link to the ASF model of participation (chapter four), this step of refining a design and preparing images of a proposal is one that did not happen in Kenya, but it was proposed that it could have been a logical next step. While some participants of the workshop debated how appropriate 'top-down' deisgn moves are in an explicitely participatory process, all agreed that drawing and visualisations are a tool in the 'expert' designer's toolkit that has a place in a participatory process.

One final reflection is that ideally, 5.4 would be followed by more engagement. If this project was to be continued, my next step would be to take these images back to tenants for another round of feedback. In this sense, it is not quite right to discuss 'top-down' work occuring following the completion of 'bottom-up' work - rather it would be better to discuss a broad participatory framework, into which some top-down design steps can be inserted.
[3] This suggests there are less substantial ways to give tenants access to computers and the internet, without necessarily installing a dedicated computer hub as some participants had suggested. 


\subsection{Developing a Participatory Approach}

This chapter is the culmination of the full body of work undertaken thus far. A set of generic principles is outlined which, it is proposed, could inform the design of a good participatory approach in any given context, or be used to assess and/or improve any existing approach. These generic principles are firstly outlined in 6.1. In 6.2, three processes are broadly tested against the principles through diagramming methods. Two of these three processes are WCC's existing approach and ASF's process employed at the Kenya workshop. The third process tested against the principles is a proposed approach for WCC. This sums up how this thesis suggests WCC could develop their approach. 6.3 then expands upon suggestions that are made for the 'WCC proposed approach'.

\subsection{Generic Principles of 'an ideal process'}

A set of eight principles is now presented. They are a product of not just my the literature review (chapter 2) but also my observations of two existing processes (chapters 3 and 4) and indeed my 'design experiment' (chapter 5). These principles cover a range of bases from who should be involved in a process (principle 1) to how a process should be planned (2), to what exercises should be included in a process (6) to how facilitators should communicate and conduct themselves $(7,8)$. These principles are deliberately simplified, generic and 'high-level' so that their application can be as wide as possible.

1. It should be ensured that participatory processes engage the widest possible range of stakeholders, as all hold keys to different aspects of a proposal.

Stakeholders should be engaged for the genuine potential they bring to improve the outcomes of a project. It may take some effort for facilitators to identify all possible stakeholders, but it is important this is done early in a process.

Origins of principle: 2.3.3 Participation cuts across traditional boundaries; 2.3.7 Participation creates a collective intelligence; Figure 3.4 Diverse consultant group; 5.1.4 Discussions over drawings

2. The scope of work to be explored through a participatory process should be left sufficiently open - at least in the beginning of a process - to ensure any 
predefined scope is able to be brought into question by above-mentioned stakeholders.

In other words, participation should occur as early as possible in the formation stages of a project so that scope is still malleable. This ensures participants have some power to influence project direction, thereby resulting in them finding more value in a process (see principle 8).

Origins of principle: 2.3.5 Processes engage power relations; 3.3.1 Context of work; 4.4.6 Considering the collective

3. Facilitators of a participatory process should have a presence at the site of a project as much as possible.

This principle is about breaking down barriers between 'the facilitators' and 'the facilitated'. It is to ensure facilitators understand as much as possible about the local realities a process is engaging with, and gives participants confidence that facilitators are at least familiar with these realities.

Origins of principle: Figure 2.2 The three pillars of PRA [see 'sharing']; 2.3.3 Participation cuts across traditional boundaries; 4.3 Participatory tools and techniques

4. Opportunities to conduct a participatory process as far along the continua (inform --> empower) as possible should be taken. 'Lower tiers' may be appropriate for certain aspects of projects, but decisions to operate here must be carefully considered.

This principle recognises that conducting processes in the higher tiers of continua generally offers far greater benefits for projects than lower tiers. It also acknowledges that it is often easier for 'power holders' to work at lower tiers, so some pressure to work further along continua may be sometimes required.

Origins of principle: 2.3.1 Processes classified along continua; 2.3.5 Processes engage power relations; 5.1.2 Semi-structured interviews 
5. Participatory processes should be of value and direct benefit to all involved, and processes should be evaluated throughout to ensure this is the case.

If a party can not see value in a process it is possible that the process is being carried out for the wrong reasons (e.g. 'to tick the consultation box'), that party's role in a process has not been made clear, or perhaps that party is not being engaged at the optimum point along a process. There is little point in conducting a process without all parties perceiving value in it for them.

Origins of principle: 2.3.2 Participation too often underdone; 5.1.2 Semi-structured interviews [see negative responses to Q3]; 5.1.5 Discussion [see 'Unproductive workshops']

6. Diverse media of participation (e.g. design workshops, interviews, etc) should be engaged throughout a process where appropriate, as they all unlock unique insights.

This principle not only ensures outcomes of a participatory process are as diverse as possible, it also ensures all people within stakeholder groups are fairly represented as different tools also resonate with different types of people. This principle also acknowledges that media engaged should match the scope of work being explored (e.g. if the extent of exterior improvements is only going to be surface finishes, it would be inappropriate to build a spatial model to explore these options. Be sure to refer back to Principle 2 in these scenarios of limited scope.

Origins of principle: Figure 2.2 The three pillars of PRA [see 'methods']; 4.3 Participatory tools and techniques; 5.1.2 Semi-structured interviews

7. Facilitators or professionals engaged in a process should communicate material in a way that is informative and engaging to lay people.

This suggests simplifying complex information into easily digestible forms and, for spatial material, favouring 3-dimensional models and clear, simple drawings over more standard representational conventions. 
Origins of principle: 4.3.5 Portfolio of options; 4.4.5 Drawing and modelling as tools; Figure 4.5 vs Figure 3.8

8. Mutual understanding of project limitations should be sought wherever possible so all participants can contribute in the most productive and useful way possible.

This principle rejects the view that participatory processes simply give people opportunities to make unrealistic requests. It argues that mutual understanding and cooperation is more productive ground for participation than ignorance, which only restricts a process from realising its full potential.

Origins of principle: 2.3.7 Participation creates a collective intelligence; 4.4.4 Building participants' capacity 


\subsection{Applying principles to three cases}

A diagramming process now explores several possibilities around how these highlevel principles might be applied to three cases. The diagrams introduce some order to the complexity of assessing or developing a process, and provide the beginnings of a language with which processes can be meaningfully evaluated and, importantly, compared. The language is graphic wherever possible, but sometimes text.

To recap, the three cases each principle is applied to are:

- WCC's existing approach

- ASF's approach at the Kenya workshop

- A proposed approach for WCC. The scenario of an upgrade of Arlington Apartments is used loosely to help illustrate how the principles might be applied to a more specific case

Certain limitations of this diagramming process should be made clear at this point. Applying the principles to a hypothetical proposed process introduces much complexity that cannot be entirely addressed in a diagram. For instance, many of the suggestions of 'WCC Proposed Approach' would require much verification before they could be implemented. These suggestions - for example, that a proposed approach should include more one-on-one meetings - are closer to the 'ideal' than perhaps what WCC's approach could be in reality. That said, this diagramming method does uncover a richness in comparison, and shows that a good process is made up of many strands and they can excel in certain areas and be insufficient in others. 


\section{Principle 1: Engage wide range of stakeholders}

It should be ensured that participatory processes engage the widest possible range of stakeholders, as all hold keys to different aspects of a proposal.

\section{Stakeholders engaged:}
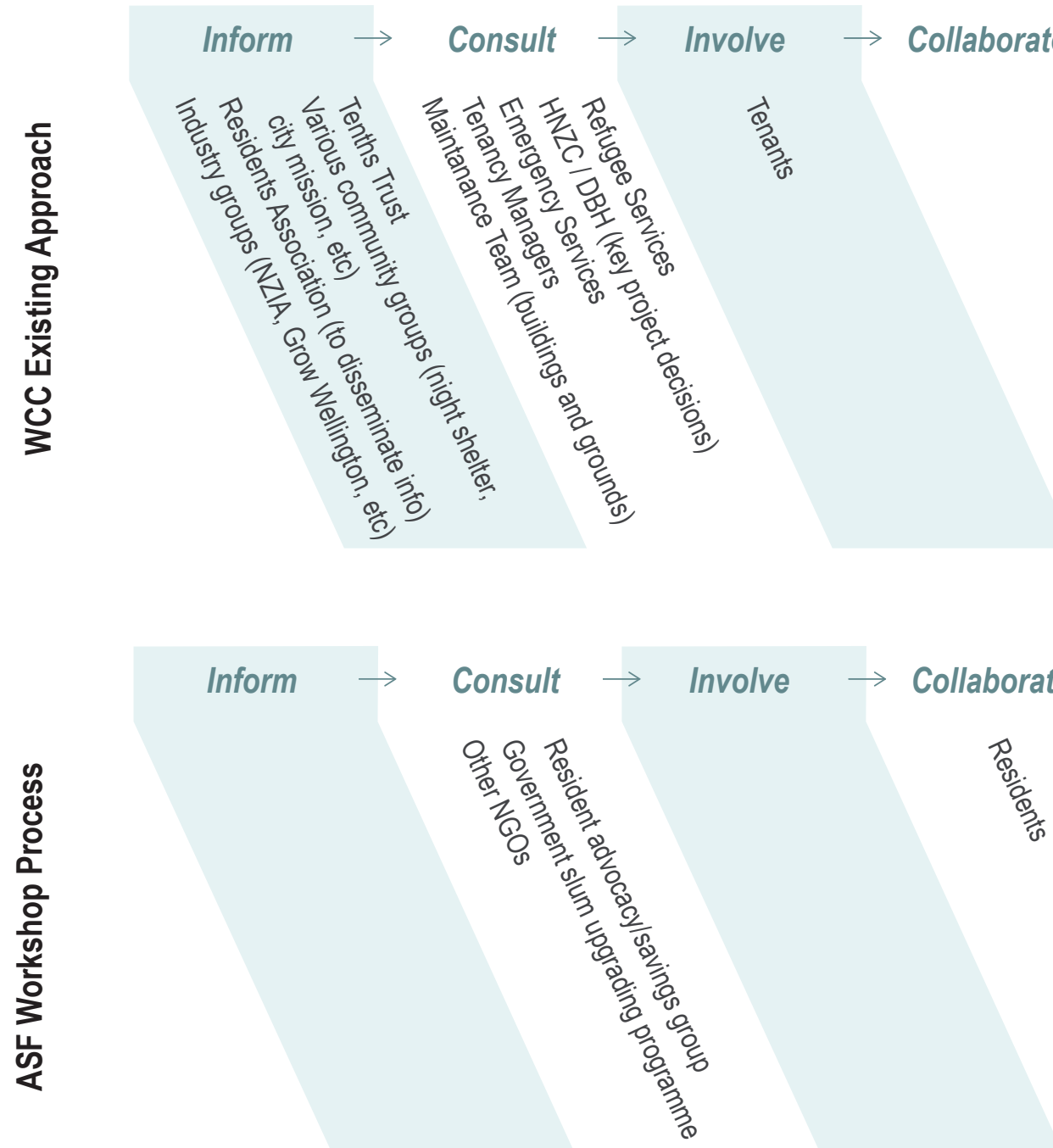

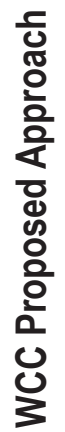

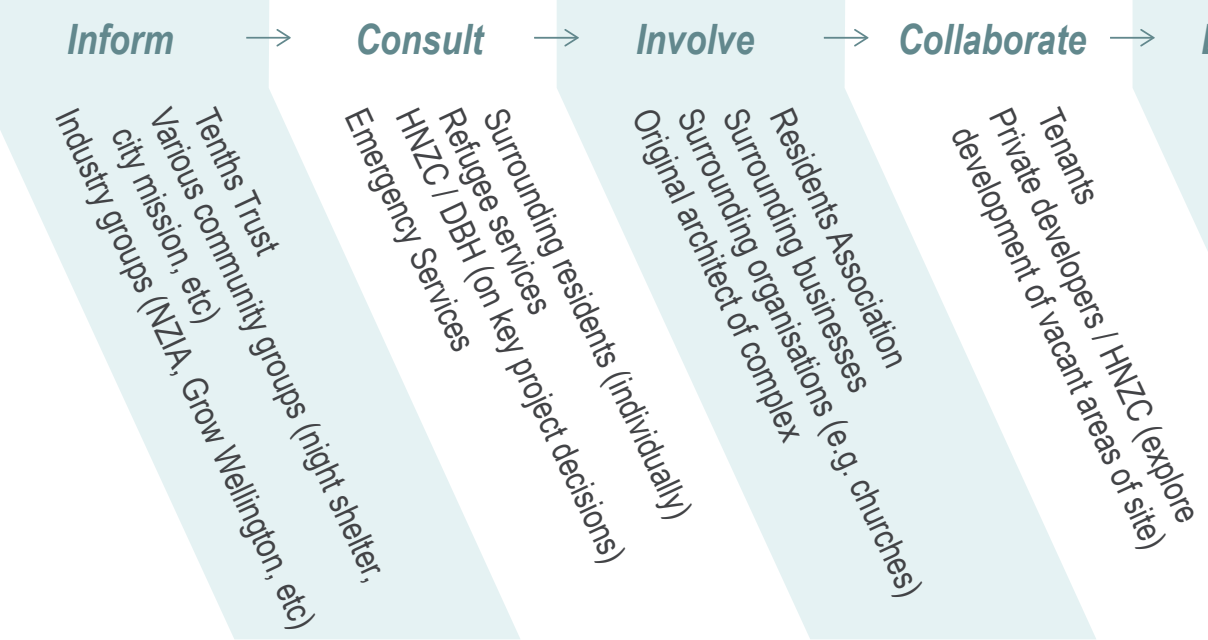




\section{Principle 2: Leave scope of work open}

The scope of work to be explored through a participatory process should be left sufficiently open - at least in the beginning of a process - to ensure any predefined scope is able to be brought into question by above-mentioned stakeholders.

\section{Participatory aspects of project in relation to defining scope: $\quad$ VIIIIIIIIIIIII/ $=\begin{aligned} & \text { participatory } \\ & \text { component }\end{aligned}$}

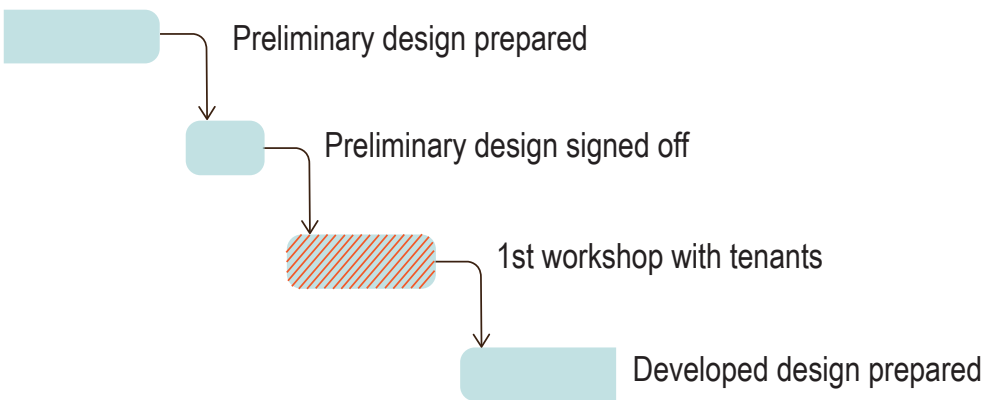

Tenant workshop not able to bring project scope into question as design already signed off.

Initial home designs prepared by residents

array of designs categorised into typologies

Typologies discussed with residents

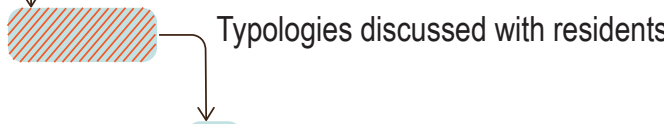

Typologies redeveloped

Etc...

Resident contributions sought throughout design phases - an iterative approach 


\section{Principle 3: Maximise on-site presence of facilitators}

Facilitators of a participatory process should have a presence at the site of a project as much as possible.

\section{Discussion on presence of facilitators}

Presence of facilitators for participatory exercises (walk-throughs, workshops, etc) was limited to the hour or two of their duration. No prolonged presence, although the series of BBQs leading up to upgrade shows efforts were made to get closer to tenants. Facilitators (WCC) have benefit of already being familiar with participants (to varying degrees) as their landlord.

Considerable amount of time spent on site allowed facilitators to have a good physical presence at all corners of the site. The sheer numbers of facilitators meant that the process could go beyond large workshops and meetings and also include more intimate home visits, which diversified findings.

It is proposed that a developed process would have a stronger presence on site than WCC's process currently does. This could even extend to a 'site office' (a vacant unit?) where work on drawings/models could take place and tenants or any other stakeholders could drop by to contribute or ask questions. 


\section{Principle 4: Move processes along continua where possible}

Opportunities to conduct a participatory process as far along the continua (inform --> empower) as posisble should be taken. 'Lower tiers' may be appropriate for certain aspects of projects, but decisions to operate here must be carefully considered.

\section{Engagement along continuum:}
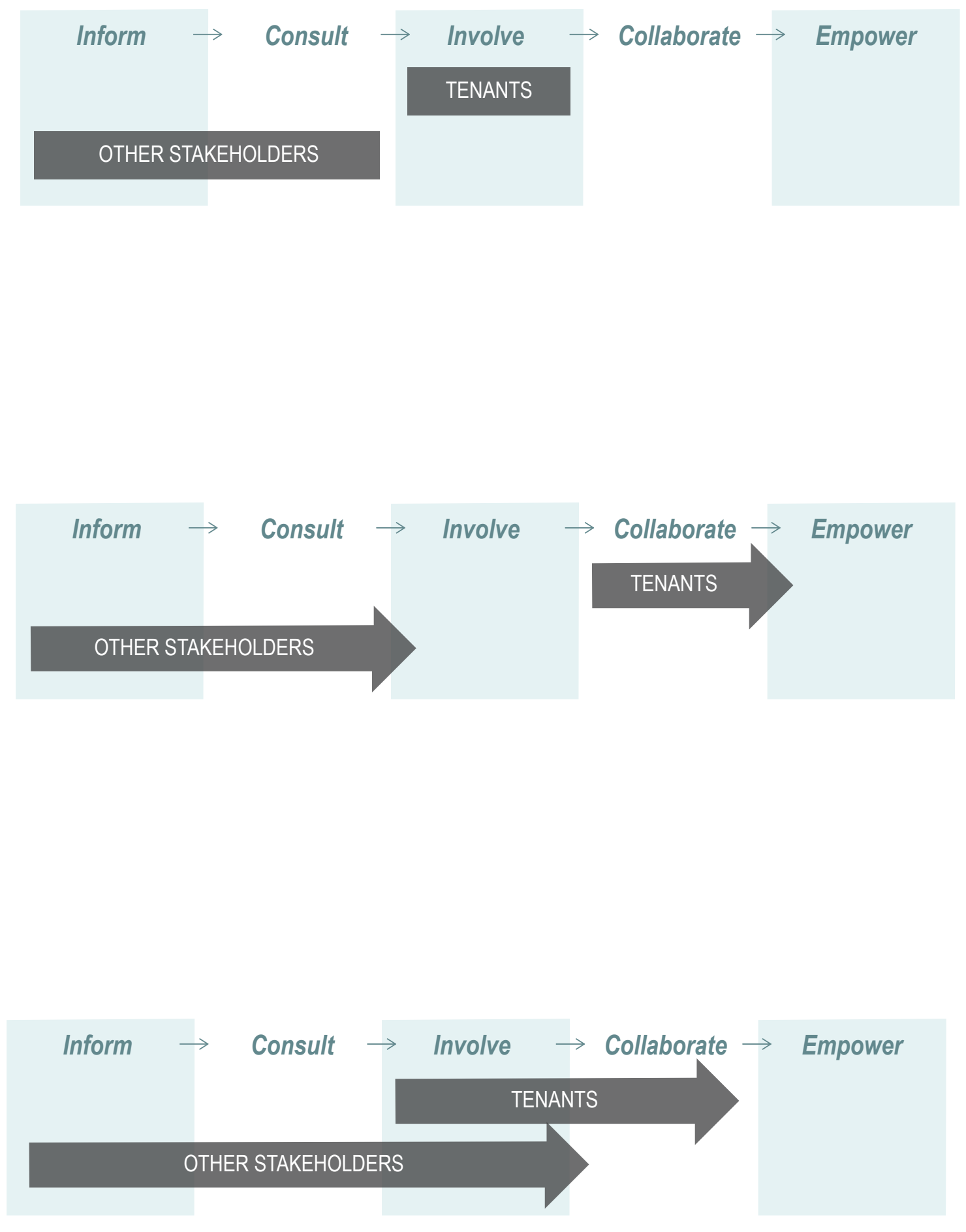


\section{Principle 5: Ensure value and benefits for all parties}

Participatory processes should be of value and direct benefit to all involved, and processes should be evaluated throughout to ensure this is the case.

\section{Discussion on value and benefits for all parties:}

Most tenants seemed to find value in the process, primarily for the sense of inclusion it facilitated.

Perceptions of value and benefits for facilitators (WCC) seem variable. Most value process as it seems right to involve tenants, although there is sceptcism about the actual tangible benefits participation brings to project.

Value gained from individual processes not explicitely evaluated throughout project duration, although broad approach to participation throughout Housing Upgrade Programme as a whole is evaluated and tweaked from project to project.

Most residents seemed to find value in the process. Participants eager for outcomes, although some doubt around direct benefits of process as actual upgrade not yet scheduled.

Very high perceived benefits of process for facilitators, whose main objective of workshop was to tap into participants views.

Value gained from process not formally evaluated throughout duration.

- It is proposed that a process be carried out that tenants value not just because they are pleased to be included, but because they the built results will directly benefit from their involvement.

Efforts will be made to ensure all components of a process have direct benefits for parties involved. To ensure this, it will be important to involve stakeholders in the design of the process itself.

Evaluation techniques (like 'exit surveys' in workshop sessions, or more casual methods) will ensure parties are finding the process valuable and beneficial at each step. 


\section{Principle 6: Engage diverse participatory media}

Diverse media of participation (e.g. design workshops, interviews, etc) should be engaged throughout a process where appropriate, as they all unlock unique insights.

\section{Media engaged:}
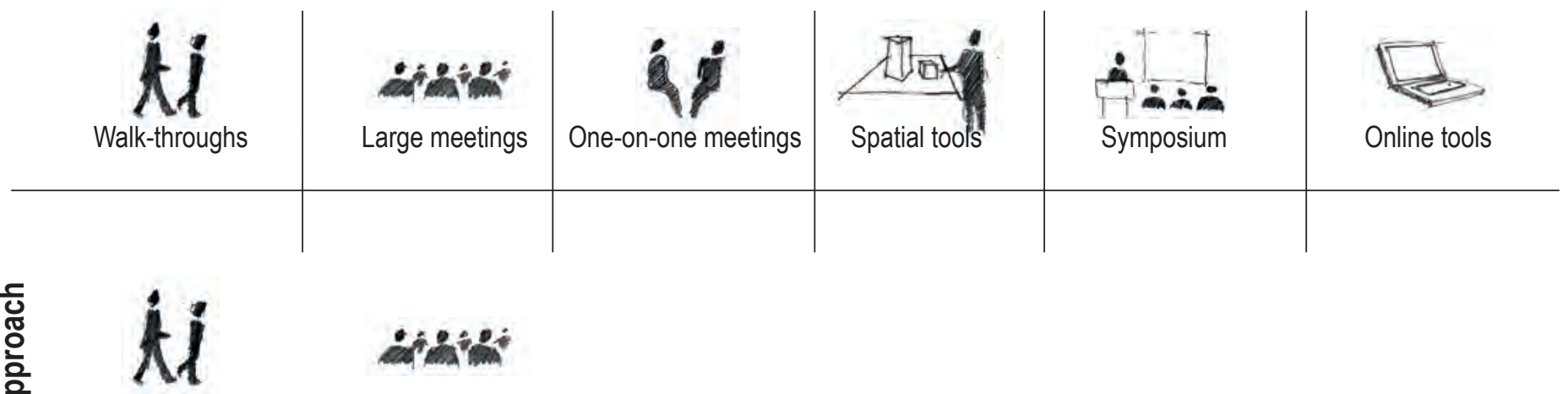

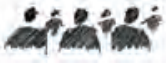

Key factors limiting the range of media engaged seem to be limited resources and perceptions that the scope of work in upgrades does not permit other tools/media. To an extent, this latter perception is justified (e.g. there was no need for WCC to build a scale model of Te Ara Hou and hold workshops with tenants in 2008 prior to the upgrade, because there were very few changes being made to the building bulk on the site). However, 'one-on-one meetings' is an example of a method that would add to WCC's process, and is appropriate given the typical scope of an upgrade.

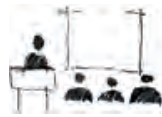

The Kenya workshop excelled in engaging diverse participatory media. Experience here has been the key informant to this principle. It is perhaps due to the educational objectives of the workshop that such an exemplary range of media were engaged, and also that such a large team of people were working so intensively for two weeks. Getting stakeholders together for a symposium is one example of a medium very distinct from others. It provided yet another forum for unique insights to be unlocked.
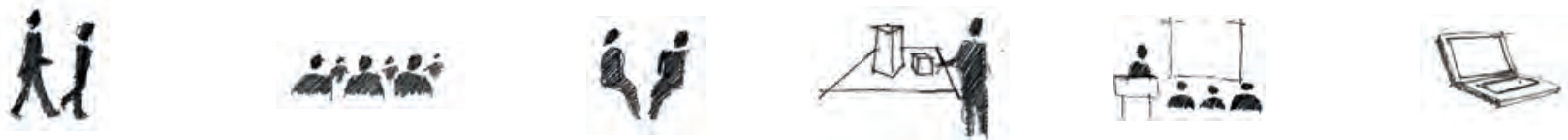

It is proposed that a wider range of media be employed in the WCC Proposed Approach. The above line-up of six media makes the assumption that an upgrade scenario at Arlington Apartments justifies each media. For example, suggesting spatial tools be employed assumes that an upgrade includes work that can be explored through such means. Similarly, a brief symposium is suggested on the assumption that proposed work has a complexity to warrent such an event (e.g. development of commercial units, infill housing, rearrangement of site planning, etc). 


\section{Principle 7: Use appropriate communication methods}

Facilitators or professionals engaged in a process should communicate material in a way that is informative and engaging to laypeople.

\section{Discussion on approach to communication:}

In all, visual communication methods employed through WCC's existing approach are insufficient. The photograph (right) shows how ineffective traditional architec-

tural drawing conventions are in communicating a proposal to a group. If the scope of a project was appropriate, 3-dimensional models should be employed as a more communicative method. Large photographs of built elements on other sites that are being repeated on a project would also be effective in communicating what is planned in a way that is accesssible to participants.

There is also a sense that complex information (e.g. available budgets, non-negotiable priorities like earthquake strengthing) is not simplified and presented to participants in a digestible form. Such information could enrich discussions around unrealistic expectations/requests some participants may have.
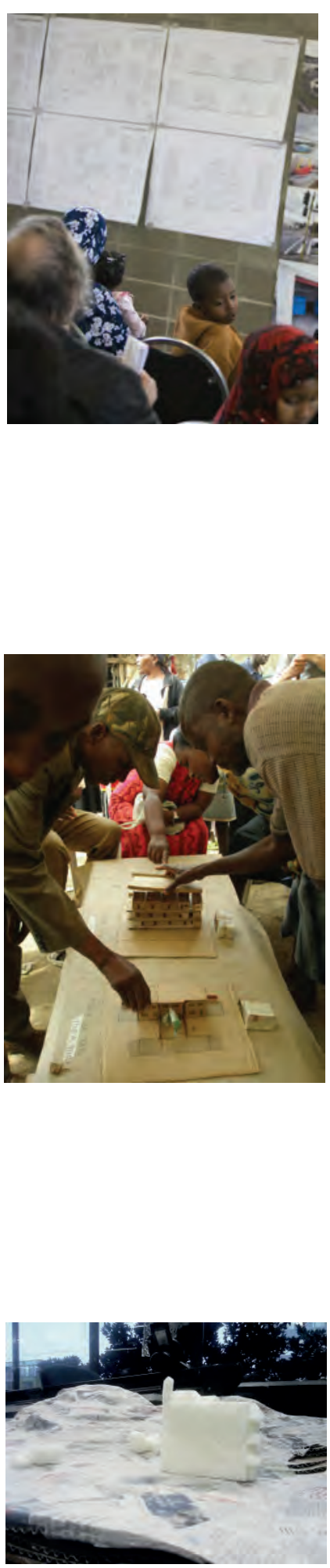

Much attention was paid at the Kenya workshop to engage communicative tools that were easy for participants to engage with. Conventional architectural drawing methods were hardly used as rough modelling methods (see photo, right) were preferred. The rough nature of models meant participants were confortable pulling them apart and rearranging elements.

The workshop was also careful to communicate complex information to participants in digestible forms. An example of this is the 'portfolio of options' exercise around a model of the settlement, where densities of different housing types were made clear as numbers on scaled 'tiles'. This allowed for quick calculations to be made on the spot to see if a proposal accommodated the required number of people.
It is proposed at Arlington that modelling techniques, similar to those used at the ASF workshop, be employed wherever appropriate as they have been proven to be most engaging for laypeople. A scale model of the site (see photo, right) could be useful to explore ideas around what to do with open spaces on the site. Larger models of a sample unit could be useful to communicate (and seek feedback on) ideas around changes to interior layouts.

A proposed approach at Arlington would be sure to present complex information like realistic scope for changes and non-negotiable priorities (e.g. earthquake strengthening) in a way that is easily digestible for participants. 


\section{Principle 8: Seek mutual understanding of limitations}

Mutual understanding of project limitations should be sought wherever possible so all participants can contribute in the most productive and useful way possible.

\section{Approaches to addressing project limitations:}

WCC staff attitudes towards the value of tenant participation in upgrades are mixed. It seems that the sceptical attitudes are (in part) due to the fact that those staff believe processes only raise tenants' expectations and give them opportunity to make unrealistic requests. This assessment speculates that more mutual understanding of project limitations would help tenants' contributions be more productive and useful for the overall projects. Yes, 'swimming pool responses' (see right) are of little use in projects like WCC's upgrades, however this assessment speculates that they only emerge from insufficient briefing/understanding about project limitations.

The Kenya workshop followed a richer approach to addressing project limitations. Our first step as facilitators seemed to be to listen to big dreams (be they even obviously infeasible). This listening process allowed participant values and aspirations to be revealed, which could then inform later steps (e.g. 'I want a large lawn and lots of trees on my section' might be an impossible request for an upgrade to deliver for every resident, however the simple process of listening to that dream from many residents could mean higher priority gets placed on some shared public park areas of lawns and trees. Following simply listening, the workshop took an approach of obtaining mutual understanding of limitations with participants, so we could all understand and move forward together (see 4.4.4 for a discussion on this in relation to housing density).

This proposed approach takes findings from the richer ASF approach and develops the conversation in HUP participation from 'what would you like to see at the complex?' (as inevitably infeasible requests ensue - see 'WCC Exisitng Approach' above) to 'here's what's roughly feasible within the scope we've got for the project at the moment - what changes would you like to see that are within that scope?' - or - 'if you feel strongly about proposals that are outside the scope we've got at the moment, can you help us think of ways the scope could be widened?'
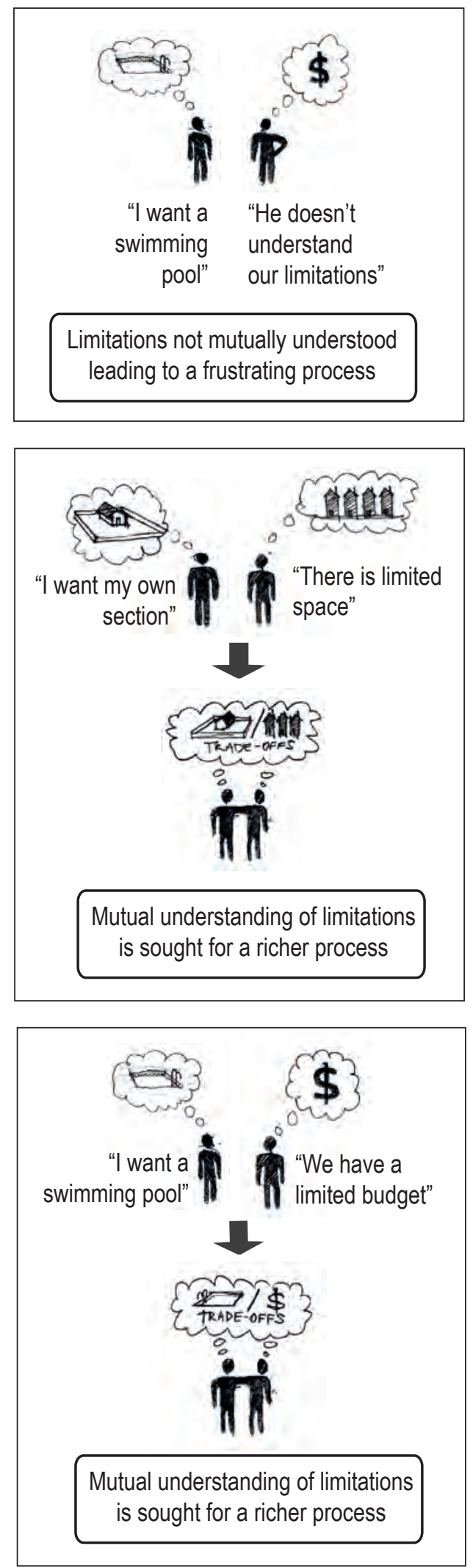


\section{CONCLUSIONS FROM APPLICATION OF PRINCIPLES}

Some brief concluding points are now made on how the processes compared in their evaluation against the eight principles. Much of the overall evaluation of the first two processes is implicit in the suggestions put forward for 'WCC Proposed Approach', which are discussed in more detail in 6.3, however this brief conclusion simply expands on some comparisons that became evident in applying the principles.

The ASF Workshop Process generally fared generally well in this evaluation as, looking back, it is evident that the experience from attending this workshop has contributed significantly to the formation of the principles to begin with. Given the generally positive evaluation of the ASF workshop process, it would be fair to infer that it is a fairly good example of 'best practice' participatory process.

Beyond this, a general theme throughout this evaluation against principles seems to be that WCC's exisiting approach is found to have room for improvement in certain areas, and in most cases it is findings from the ASF process which inform how it should be developed (into 'WCC Proposed Approach'). It is worth reiterating that what is suggested as WCC's 'Proposed Approach' is perhaps closer 'the ideal' than what could be implemented in reality, and a process should always be designed in response to a particular project. Particular circumstances of a project may make some of these suggestions irrelevant, in which case the higher-level principle should be reverted back to and reinterpreted.

In all, the method of applying the principles to three process simultaneously proved insightful. Comparison was particularly rich in principles that assessed processes along a spectrum or across a time scale, like principles one, two and four. 


\subsection{Expanding on WCC Proposed Approach}

This sub-chapter elaborates on some of the thinking behind suggestions made for the 'WCC Proposed Approach' through the previous diagramming exercise. These additional thoughts are grouped under each principle below. Following that, the proposed approach is presented in the same diagramming language developed for the earlier two processes in chapters three and four.

- Principle 1: Engage wide range of stakeholders

Some of the suggestions made here are based on the speculative scenario that there is scope at Arlington to develop additional housing. Given this proposed scope of work, a wider range of stakeholders is proposed to be engaged. Private developers and/or Housing NZ Corporation are proposed to be collaborated with to explore where new housing could be sited (see Figure 6.1). This could be seen as an opportunity for City Housing to cash in on high opportunity costs of land, or perhaps an opportunity to respond to Wellington projected increasing need for 'social housing' units into coming decades (identified in 3.3.2). Residents associations are also proposed to be engaged for more than simply disseminating information (as they are currently), as the site has the unique urban condition of straddling a public street which increases the importance of the 'surrounding residents' stakeholder group. It is also proposed that the original architect of the complex (lan Athfield) be involved in an upgrade, as he could offer unique insights (based on decades of experience since designing the complex) into what aspects of the design could be tweaked to improve certain conditions.

Figure 6.1: Site Plan (N.T.S) of Arlington Apartments showing possible infill opportunities

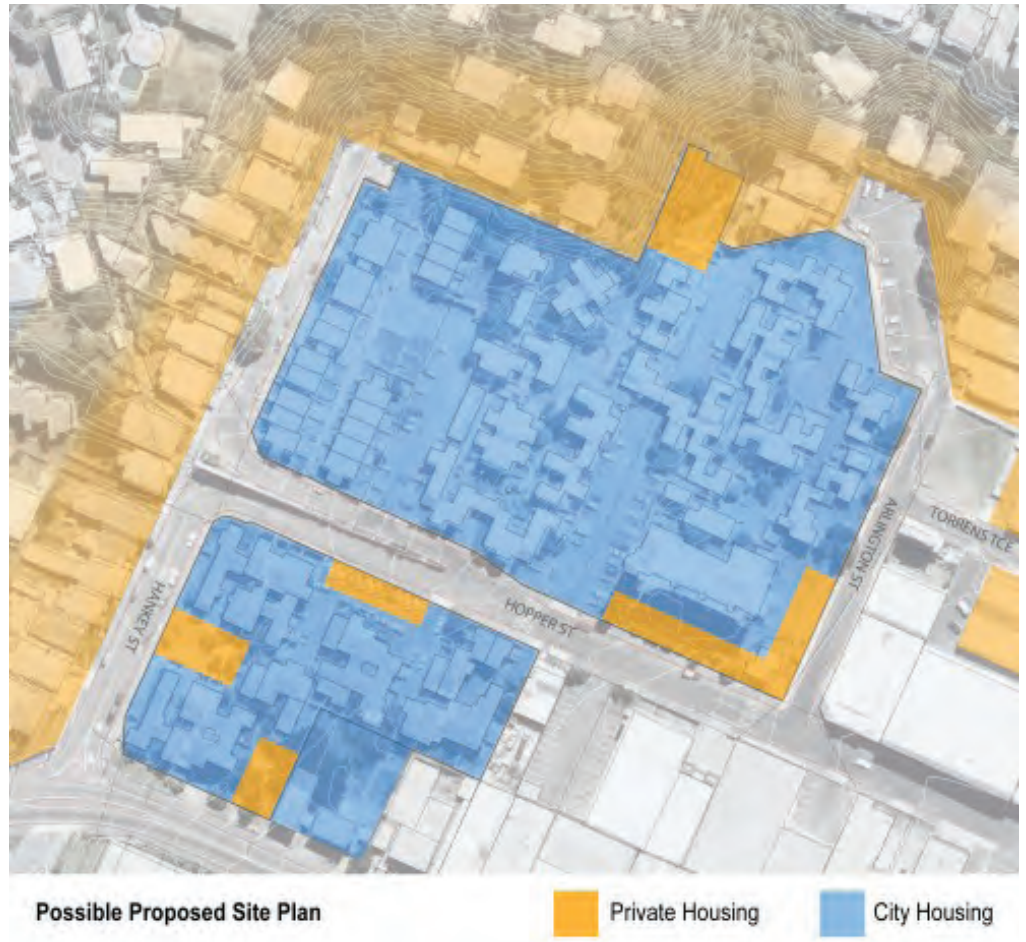


- Principle 2: Leave scope of work open

A more integrated approach to participatory components of the process is proposed. This contrasts the current approach which contains participation to set periods. Involving stakeholders at all stages, especially early stages when project parameters are defined, is important so participants' views can influence this critical stage.

- Principle 3: Ensure on-site presence of facilitators

These suggestions take lessons from the Kenya workshop. A more sustained presence is proposed over a longer period of time in order for facilitators to get as close to 'local realities' as possible. The challenge of resourcing a stronger presence is discussed in 7.3.

- Principle 4: Move processes along continua where possible

Generally, the proposed process operates further along the 'inform-to-empower continuum' than WCC's current approach. Arrowheads in the diagram are an important representation the constant pressure to push stakeholders along the continuum wherever possible. For tenants, there is a general push from 'involve' (their position in current process) to 'collaborate' which has the promise "we will look to you for advice and innovation" (see Figure 2.5 - IAP2 Spectrum). Other stakeholders are proposed to be involved for the value they may bring, rather than simply consulted or informed.

- Principle 5: Ensure value and benefits for all parties

The proposed process engages all stakeholders on the basis that there is some value and benefit to them, and this is emphasised. Wherever possible, scepticism from facilitators or participants about a process will be addressed by re-working the process until value and benefit is present for all. The process also endeavours to be constantly reflexive, to ensure participants and facilitators are finding it valuable and beneficial throughout.

- Principle 6: Engage diverse participatory media

ASF's process influences the proposed process heavily here. Three 'participatory media' from that workshop are proposed to be adopted: one on one meetings, spatial tools and a symposium. One-on-one sessions are proposed as they tap into learnings other methods miss. Spatial tools are very important, especially if the idea of infill developments is pursued as they have been found to be particularly engaging for lay people. Thirdly, a symposium-type event is 
proposed, as in Kenya, to bring collective thought together (see Figure 6.2). This could be a small event (perhaps only two hours) on site, where some tenants present a quick-fire range of views, architects present their diagnoses, historians present their assessments of the site (e.g. see McEwan and Bowman), surrounding businesses present their key challenges, etc. Discussions around ways forward begin as a collective. Finally, online tools (e.g. blogging) are proposed to be trialled, to potentially facilitate a wider reach of participants and engage important younger generations.

Figure 6.2: Symposium at UN-HABITAT (Kenya) to develop collective thought from a range of stakehold-

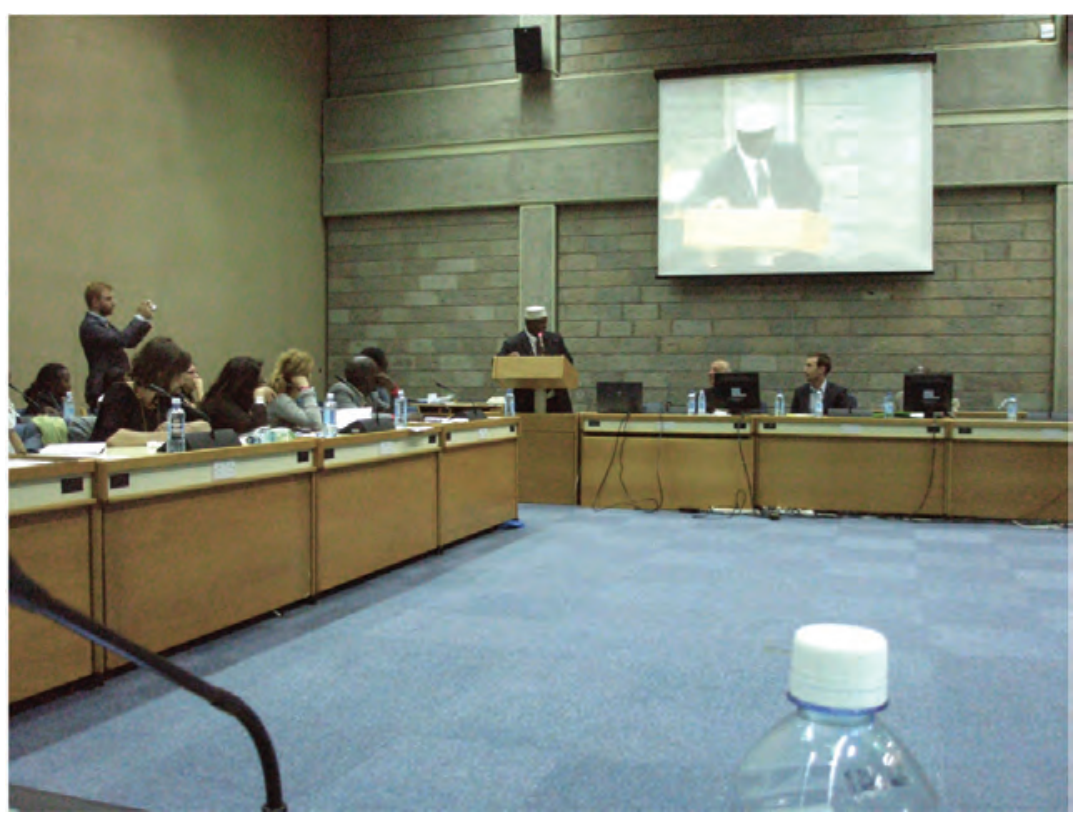

- Principle 7: Use appropriate communication methods

The proposed approach favours modelling as the communication method for spatial information. It will also strive to communicate complex information in a way that is understandable and engaging so that all involved in a process can share a reasonable base-level understanding of project parameters. This might involve coloured pie graphs/charts about available budgets left after non-negotiables are paid for, etc.

- Principle 8: Seek mutual understanding of limitations

Mutual understanding of limitations will be sought wherever possible. This is having learnt a lesson from WCC's existing process, which struggles with how to deal with unrealistic requests/expectations from participants. This process takes the position that in order to maximise usefulness of inputs from all participants, this mutual understanding is critical. 
In all, chapters 6.2 and 6.3 propose a new improved participatory approach for WCC that, this thesis proposes, could deliver even better upgrades to complexes. It is worth reiterating, too, that many of the above suggestions are made on the assumption that scope of projects would justify more intensive participatory process. The question is now asked, 'what main benefits would this new improved process deliver the results of an upgrade?' In response to this, the first main benefit the thesis puts forward is that by engaging a wider range of stakeholders (like developers, surrounding businesses, residents, etc) this improved process would help deliver upgrades that are better for the city at large. In other words, the current approach is delivering good results for WCC, but in an improved process lies an opportunity to go beyond needs of WCC and deliver upgrades that respond to needs beyond these like, for example, a pressing social housing shortage the city is facing (see 3.2.2) or needs a street might have for small commercial units to be developed along a street edge. Secondly, a process that works more closely with tenants will deliver an upgrade that better reflects their needs and aspirations for complexes. This will have a direct positive effect on how they carry out their lives. Thirdly, and related to this, an improved approach offers an opportunity to further develop tenants' connection to a complex which generally increases their readiness to protect and enhance their place. This, in turn, reduces long-term maintanance demands.

Of course, a more intensive participatory process carries costs and certain risks. Risks include increasing complexity of upgrades making them take longer to plan, and a rising of expectations in stakeholders that might not be able to be met. To the latter risk, Wates candidly reminds us to keep in mind that "nothing much is likely to be achieved without raising expectations" (2000:13), and it should be remembered that rising expectations is something that can be managed.

Whilst these projected benefits carry the limitation that they have not been tested at a un-upgraded complex (like Arlington Apartments) - and therefore are not yet firmly proven - they are put forward with a degree of confidence as they are informed by findings from previous chapters of the thesis.

This propsoed approach for WCC is now presented in an overall diagram overleaf. 
WCC Proposed Approach

\section{EVENTS}

Social events with tenants

Mini-symposium

Walk and Talk sessions

Activate - workshops series

\section{Tenants}

ideas for interiors/exteriors

Surrounding residents matters that affect them

Developers / HNZC opportunities for infill dvpt

Activate - reporting back

Activate - open day / exhibition

\section{KEY OUTCOMES}

Base level collective understanding of challenges specific to project
Ideas and developed responses for project from various perspectives

The proposed approach takes the basic structure of WCC's exisiting approach and adds various additional events. The addition of a 'mini-symposium' responds to a successful component of the ASF workshop (see 6.3: Principle 6). It is also proposed - where scope of a project allows - that the stakeholder group engaged is made wider, by running a series of Activate workshops with various groups (see 6.3:Principle 1)

Figure 6.3: Overall diagram of WCC Proposed approach.

Similar diagrams for the WCC Exisitng Approach and ASF Workshop Process are repeated from earlier chapters (see right) for comparison. 


\section{WCC Existing Approach}

\section{EVENTS}

Social events with tenants

Walk and Talk sessions

Activate - workshop

Activate - reporting back

Activate - open day

\section{KEY OUTCOMES}

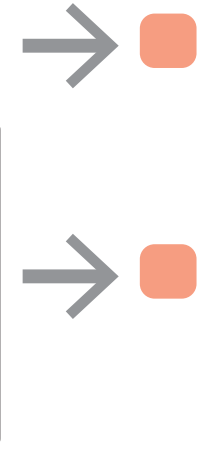

Report to consultants

Brief reports to consultants. Consultants also present at events to absorb tenant views

\section{ASF Workshop Process}

EVENTS

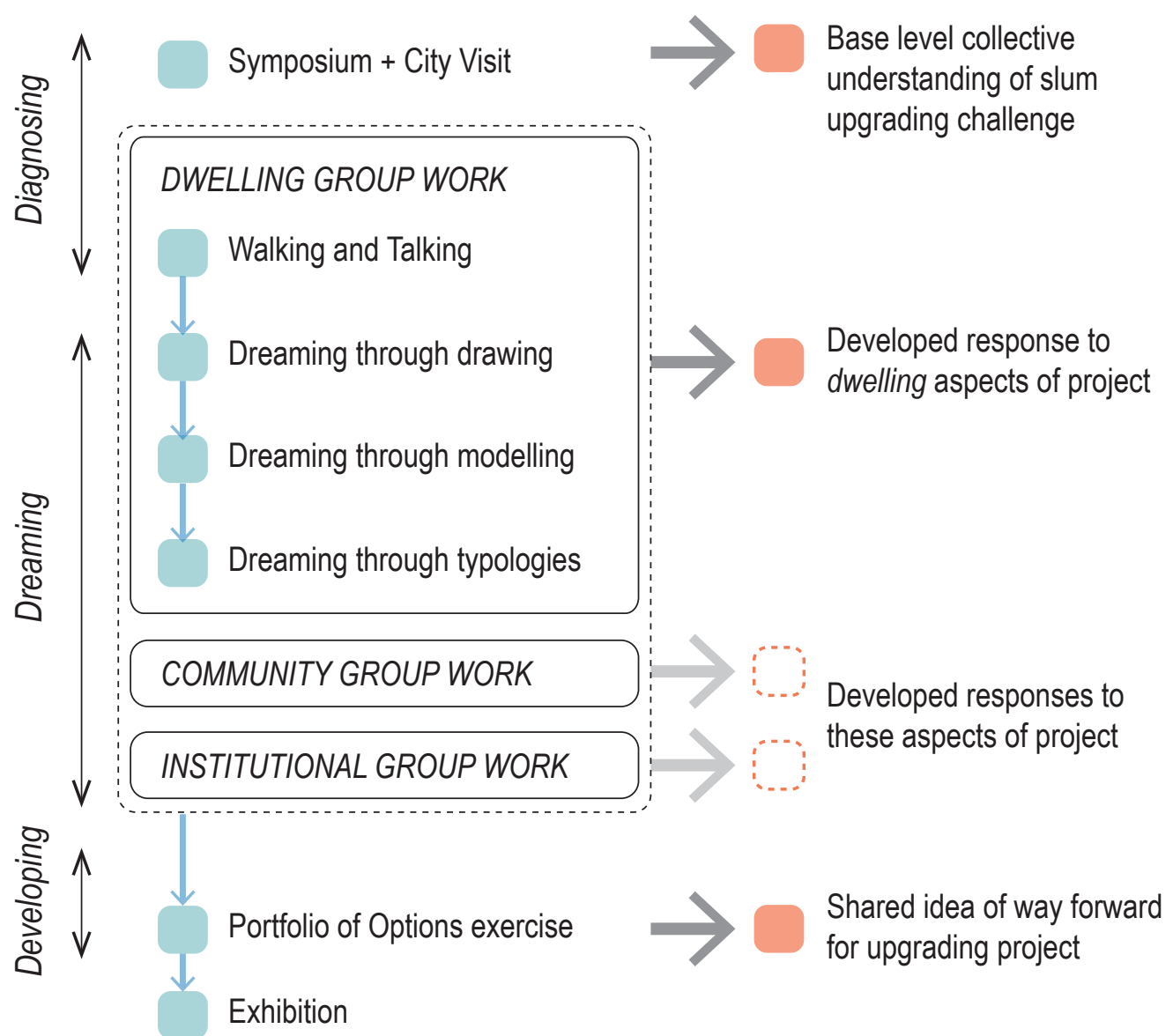




\subsection{Overall Conclusions and Reflections}

Chapter seven offers some overall conclusions and reflections on the study as a whole. As many findings, limitations and reflections have already been discussed at the conclusion of each chapter, this chapter is concise in its summary of key points. Firstly, the study as a whole is summarised in terms of initial research questions and research methodology followed. Then key findings are summarised including some discussion on their implications for research and practice. Some limitations of the research are then outlined, followed by suggestions of future directions.

\subsection{Summary of study}

The project found that yes, there is scope for widening tenant participation in WCC's Housing Upgrade Programme. The pursuit of an answer to that initial question began with a literature review which sought to understand what constitutes 'best practice' participation. This revealed a loose set of seven points common across a variety of sources which broadly define participation. A comparative analysis of two participatory process - WCC's process in their Housing Upgrade Programme and that of the ASF workshop held in Kenya - then ensued. The look at WCC's process included some background on social housing in New Zealand to place this study in a broader context. To close this comparative analysis, discussion points were made on the Kenya workshop referring also to WCC's process. 'A design experiment' was then carried out which tested certain participatory exercises at Te Ara Hou Apartments in Newtown, Wellington, followed by a more top-down design process which explored how a more conventional approach to design slots into a participatory approach. All of these lines of enquiry then led to chapter six which proposed a generic framework for 'developing a participatory approach.' Broad recommendations are made around how WCC could develop their participatory approach.

The project found methodological basis in the tradition of action research, and followed a case study method at various points. The project has sought "to bring together action and reflection, theory and practice, in participation with others, in the pursuit of practical solutions to issues of pressing concern to people" (Reason and Bradbury 2006:1). 


\subsection{Key findings and limitations}

\section{EIGHT GENERIC PRINCIPLES}

The lead question "is there scope for widening tenant participation in WCC's Housing Upgrade Programme" has been answered more broadly than was initially anticipated. In summary, the thesis finds that yes, there is scope for widening tenant participation. Eight generic principles developed in chapter six elaborate on different aspects of a participatory process, and in some cases go beyond the consideration of only tenant participation. These are:

- Principle 1: Engage wide range of stakeholders

- Principle 2: Leave scope of work open

- Principle 3: Ensure on-site presence of facilitators

- Principle 4: Move processes along continua where possible

- Principle 5: Ensure value and benefits for all parties

- Principle 6: Engage diverse participatory media

- Principle 7: Use appropriate communication methods

- Principle 8: Seek mutual understanding of limitations

Principles are deliberately abstract because I realised I couldn't pin down detailed suggestions without knowing the particularities of a given project scenario. Their abstract nature also ensures the outcomes of this project can be applied more broadly to other participatory settings. Although I concede that due to the complexity of this topic such a list of principles could never be exhaustive, I do think that the approach of applying principles across contexts is helpful. I suspect that like Chambers' own development of his 'three pillars of PRA' (see Figure 2.2), such a list of principles could be developed over time as my (and others') understanding and practice of participation grows further.

\section{COMPARISON OF PROCESSES FRUITFUL}

In all, it has been striking how directly comparisons have been able to be made between two very different participatory processes. The comparative analysis between ASF's Kenya workshop process and WCC's HUP process revealed a set of discussion points (4.4) that applied to both processes. In brief, that participatory work can be time consuming and its benefits difficult to measure was something found to be common across both processes. It also became clear that regardless 
of the context, participation is an inherently social activity that engages power dynamics whether they be within groups of participants or across participant-facilitator lines. The nonphysical benefits of participation (like a developed capacity to understand development challenges or increased attachment to place) became hard to ignore when considering the benefits of a process. In both Kenya and Wellington, too, it became clear that it is important to employ a wide range of participatory tools as they all deliver unique benefits and outcomes. What made the comparison of the two processes fruitful was that it occurred at a sufficiently high level. If exact specifics and differences of the two settings were focussed on, common ground between them would be harder to find.

\section{A PLACE FOR TOP-DOWN DESIGN PROCESS}

The project found that the more conventional 'top-down' approach to design in 5.2 ultimately complimented the 'bottom-up' participatory work that had gone before it. In all, the thesis confirms that a call for a rise in the status of lay people in a design processes certainly does not diminish the role of professional architects/designers; indeed it only places further importance on their skills. Some of those skills that became increasingly apparent through this study include the need to negotiate a wide range of stakeholder interests and also to communicate architectural/ spatial ideas in ways that are accessible for lay people. Zeisel's cartoon below highlights the need for architectural representation to express people's ideas:

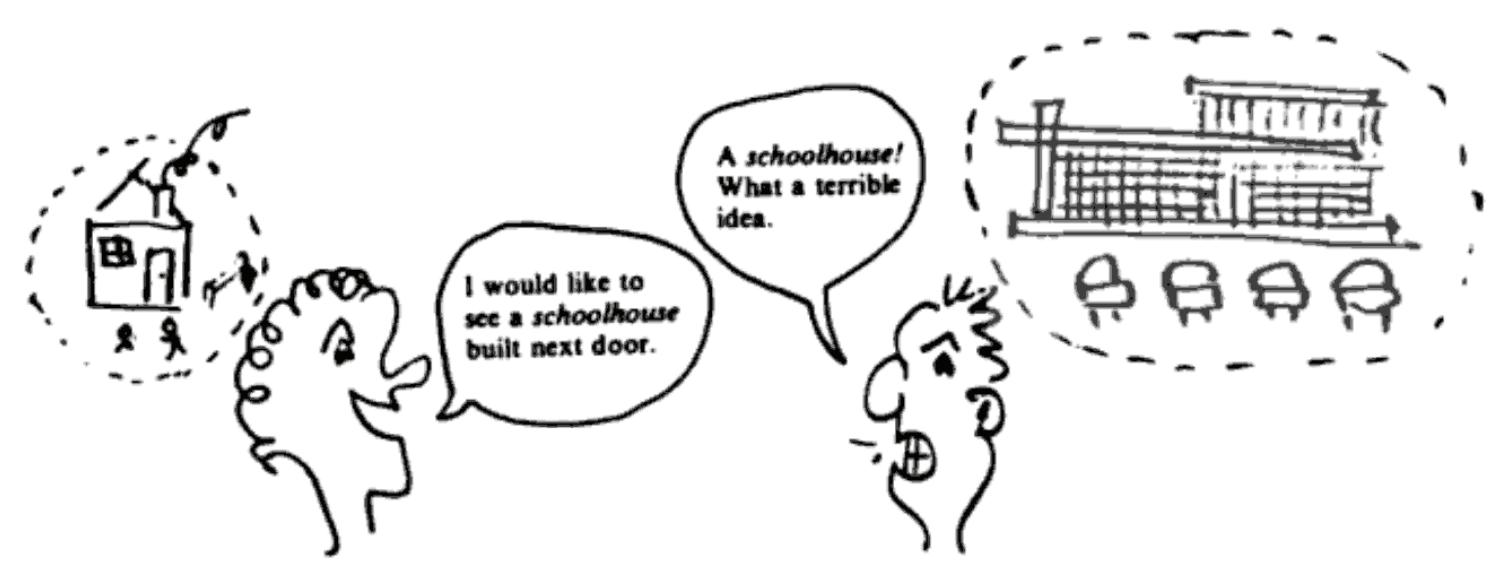

Figure 7.1: 'Negotiating a shared image' (Zeisel 2006:50)
Certain aspects of development work remain in the domain of architects, therefore participation need not be thought of as 'professionally threatening' as it so often is (Hamdi 2010:98). Dong puts this eloquently: "what is needed is not user participation in design as a counter-force to the power of designers ... but instead a design culture of pluralism with effective means for achieving it" (2008:77). To a degree, this project has been a successful example of this pluralistic approach, although 
I do concede that this project falls short in that due to time constraints, I was not able to return back to participants with the drawings presented in 5.4 for another round of discussion, as would have been best practice.

\section{FAMILIARITY OF CONTEXTS - EFFECTS OF A LANGUAGE BARRIER}

Although the English-speaking facilitators of the Kenya workshop did have the assistance of local students to translate interviews and workshops from Swahili, the language barrier was still somewhat restrictive to the work we undertook. Whilst I do not fully align with the somewhat extreme view of Bill Cooke that practitioners should "work only in languages [they] understand as well as their first", (rule IV of his 'rules of thumb for participatory change agents' - Cooke 2004:48), I do acknowledge the limitation a language barrier causes. Natural flow of conversations was constantly interrupted as interviews and workshops were translated every few sentences, which did slightly restrict us. The absence of a language barrier for my work at Te Ara Hou in Wellington meant I focussed more heavily on the conversational methods like semi-structured interviews, as I was able to engage in good discussions with most participants. On reflection, I can see that one downfall of this ease of conversation is that participatory methods involving drawing and modelling are called upon less, which means some findings are potentially missed.

\section{MONITORING APPROPRIATENESS OF PARTICIPATION}

Cooke's Rule VI of his 'rules of thumb for participatory change agents is 'have it done to yourself' is something I had in the back of my mind throughout my work at Te Ara Hou. I was sure to constantly ask myself, 'would it be acceptable if someone knocked the door of my home and asked me the kinds of things I'm asking?' This "undergoing the procedures we inflict on others" (2004:51) is good practice, Cooke argues, as a baseline test that our work is respectful, noninvasive and nonexploitative of the marginalised. I would carry this point forward into any future work I'm involved with.

\section{KEY LIMITATION OF 'WCC PROPOSED APPROACH'}

An obvious limitation of the 'WCC proposed approach' put forward through the application of the eight principles in 6.2 is that this approach is partly speculative and can not be firmly proven as it has not been tested at an un-upgraded complex. While suggestions are put forward with a degree of confidence as they are 
informed by findings from previous chapters of the thesis, they would ultimately need to be tested before it could confidently be said that they represent an entirely feasible, improved approach for WCC. Another key characteristic of the proposed approach that limits its wider applicability (in its entirety) is that it is based on a scenario of much broader scope than WCC's typical upgrades. Many of these suggestions would not apply to an upgrade that simply refurbished existing buildings. This points to a wider finding of the study that a participatory approach will always have to be designed to match the scope of any given project. It the scope of a project is reduced, then an approach should be scaled down to match it (i.e. don't hold a modelling workshop to choose paint colours). 


\subsection{Future Directions for Project}

I offer the following four reflections on future directions for the project. The first offers an idea as to how recommendations of this study could be implemented by WCC, and the remaining points suggest possible future avenues that could follow on from this study.

\section{REDUCING COSTS OF PARTICIPATION}

A proposition to widen the participatory programme within the Housing Upgrade Programme is likely to be met with the understandable response that resources within the programme are already stretched and it would be difficult to justify more spending on participation. Engaging with this criticism is critical for the project to move forward. One solution, I believe, lies in the harnessing of 'free labour' of research students at universities. This represents a considerable opportunity to bring the labour costs of participation down whilst also delivering participatory researchers a key pedagogical element to their study - field experience. In conjunction with professional consultants, and ideally through an established participatory research unit at a university, ${ }^{1}$ partnerships between councils (/government departments) and research students offer great potential to be mutually beneficial for all parties.

\section{WCC COMMUNITY SPACES}

One area of the research that warrants further investigation is the uptake and success of existing community facilities that are provided within several of the WCC housing sites. Although my study of four existing community spaces sufficiently thorough for the purposes of this study, it could be further extended by investigating the spaces from perspectives other than WCC staff. Interviews with community room coordinators and, importantly, randomly selected tenants of the complexes would potential reveal differing views to those of WCC staff. Less subjective means of investigating how the spaces are used could involve analysis of spaces' booking schedules and empirical methods of trace observations to examine what occupants do both in and to environments (Zeisel 2006:159-190). Such research could better inform how future community spaces are developed.

[1] The Development Planning Unit (DPU) within University College London (UCL) is one such example of an established unit that focuses on participatory programmes: http://www.bartlett.ucl. ac.uk/dpul 


\section{ATTITUDES TOWARDS AND VALUE OF PARTICIPATION}

I believe there is room in the body of literature for a lot more work like Warburton's 2005 study, 'Is participation too expensive?', which begins to quantify the benefits of participation and therefore give it more basis on which it can be advocated for. This study has focussed largely on the qualitative benefits of participation, however it would now be useful to follow this up with qualitative analysis. For example, it would be interesting to take a qualitative finding of this study - that participation improves residents' attachment to place and therefore makes them more keen to protect it - and follow it up with a study of numbers of maintenance-from-vandalism call outs and instances of graffiti in complexes that have been upgraded with resident participation.

Related to this, it would be interesting to survey built environment professionals' attitudes to the participation of lay people in a design process, to confirm or deny (and elaborate on) the often referred to, but largely unfounded, claim of this thesis that mainstream practice feels a general apathy towards participation. It would be interesting to unpick these views to understand why, if at all, this is the case.

\section{GAP IN LITERATURE - PARTICIPATION IN DEVELOPED WORLD}

Generally, this thesis found most of the lively discussion and critique of participatory processes to be in reference to participatory practice in the developing world. The large field of 'international development' incorporating countless non-profits, NGO's and institutions like the UN and World Bank and a strong academic culture seem to keep the shelves of 'participation in the developing world' well stocked (e.g. Cooke and Kothari 2001; Fisher 2001; Hickey and Mohan 2004). On the other hand, literature on participatory process in developed countries, grappling with admittedly less impoverished contexts but equally fraught with challenges, seems to have more gaps. This thesis has proven that there is much to be learnt and transferred across contexts, however many conditions are unique to developed countires and warrant further discussion. There is much light 'user-manual-style' literature circulating for people running participatory programmes (e.g. Planning Aid England 2010; Wates 2000, 2008), however this work avoids deeper theoretical/ideological discussion. Stronger discussion on the realities and challenges of participatory design in the developed world - to the level of rigour that work in the developing world is debated at - would assist those working in these contexts and would further fill what I perceive to be a gap in the wider body of literature. 


\subsection{Final Statement}

This thesis asked the question, 'is there scope for widening tenant participation in Wellington City Council's Housing Upgrade Programme?'. The question has been addressed through a multi-threaded investigation which led to the development of a set of generic principles which can be applied in any number of contexts. The ultimate answer to the question is yes, there is scope for widening tenant participation, and points are made on many other aspects of a participatory approach too.

Tenants alone don't know the best way to develop sites. Council staff alone don't know the best way to develop sites either. Nor do architects or other professionals who are unfamiliar with sites, for that matter. This thesis argues that it is through the effective coming together of various parties that the best ways forward can be established, and tenants are an important part of that equation. Developing a participatory approach is about simply tapping into those parties' contributions and bringing them together in an effective way.

Chambers states that "good workshops are ... like a sea voyage... [one should] go with the flow, roll with the punches, and steer by sailing and tacking with the wind" (2002:xiv). This thesis has sought to develop the base-level knowledge required to undertake such voyages into the future. 


\section{Bibliography}

Arnstein, Sherry. (1969) A Ladder of Citizen Participation. American Institute of Planners Journal. July p. 216.

Atlee, T. (2003) The Tao of Democracy: Using Co-Intelligence to Create a World that Works for All. Cranston: The Writers' Collective.

Chambers, Robert. (2002) Participatory Workshops: a sourcebook of 21 sets of ideas and activities. London: Earthscan.

Chambers, Robert. (1997) Whose reality counts? Putting the first last. London: Intermediate Technology Publications.

Cooke, Bill. (2004) Rules of thumb for participatory change agents (pp. 42-55). In Hickey, Sam and Giles Mohan (eds). Participation: from tyranny to transformation? Exploring new approaches to participation in development. London: Zed Books.

Cooke, Bill and Uma Kothari. (2001) The Case for Participation as Tyranny (pp. 1-15) in Cooke, Bill and Uma Kothari (eds). Participation: The New Tyranny? London: Zed Books.

Cornwall, Andrea. (2004) Spaces for transformation? Reflections on issues of power and difference in participation in development (pp. 75 - 91). In Hickey, Sam and Giles Mohan (eds). Participation: from tyranny to transformation? Exploring new approaches to participation in development. London: Zed Books.

Dong, Andy. (2008) The Policy of Design: A Capabilities Approach (pp. 76-87). Design Issues. Vol. 4. No. 24.

DTZ New Zealand and Stimpson \& Co. (2006) Wellington City Housing Needs Assessment. Wellington: DTZ New Zealand.

Eisenhardt, Kathleen. (2002) Building theories from case study research (pp. 5-35). In Huberman, A. Michael and Matthew Miles (eds). The qualitative researcher's companion. London: Sage Publications.

Ellis, John and Julia Kiely. (2005) Action Inquiry Strategies: taking stock and moving forward (pp 85-100). In Cooke, Bill and Julie Wolfram Cox. Fundamentals of 
Action Research: Volume IV. London: Sage Publications.

Fisher, Fred (UNCHS). (2001) Building Bridges [between citizens and local governments to work more effectively together] Through Participatory Planning. UN Habitat. <http://www.unhabitat.org/pmss/getElectronicVersion.asp?nr=1371\&alt=1> Accessed 19.02.12.

French, Matthew (ed). (2011) Change by design: building communities through participatory design. Urban Culture Press: New Zealand. [Available for download: $<$ http://www.scribd.com/doc/75033019/Change-by-Design-Building-Communities-Through-Participatory-Design>]

Gaventa, John (2004). Towards participatory governance: assessing the transformative possibilities (pp 25-41). In Sam Hickey and Giles Mohan (eds). Participation: from tyranny to transformation? Exploring new approaches to participation in development. London: Zed Books.

Hamdi, Nabeel. (2010) The placemaker's guide to building community: planning, design and placemaking in practice. London: Earthscan.

Hickey, Sam and Giles Mohan. (2004) Towards participation as transformation: critical themes and challenges (pp 3-24). In Sam Hickey and Giles Mohan (eds). Participation: from tyranny to transformation? Exploring new approaches to participation in development. London: Zed Books.

Housing Shareholders Advisory Group (HSAG). (2010) Home and Housed: A Vision for Social Housing in New Zealand. <http://www.dbh.govt.nz/UserFiles/File/ Publications/Sector/pdf/vision-for-social-housing-nz.pdf> Accessed 03.02.12

Huberman, A. Michael and Matthew Miles (eds). (2002) The qualitative researcher's companion. London: Sage Publications.

Jacobs, Jane. (1961) The Death and Life of Great American Cities. Random House: New York, 1993.

Jenkins, Paul. (2010) Concepts of Social Participation in Architecture. In Paul Jenkins and Leslie Forsyth (eds). Architecture, Participation and Society. London: Routledge. 
Madge, John. (1968) Housing: Social Aspects (pp. 516-517). In D. Sills (Ed). International Encyclopedia of the Social Sciences 6. New York: Macmillan \& The Free Press.

McEwan, Ann and lan Bowman. (2010) Arlington Apartments: Heritage Record and Assessment. [Held at WCC].

Ministry for the Environment. (2009) Urban Design Toolkit. 3rd ed. <http://www. mfe.govt.nz/publications/urban/urban-toolkit-2009/urban-design-toolkit-third-edition.pdf> Accessed 11.11.11.

McNiff, Jean and Jack Whitehead. (2002) Action research: principles and practice. 2nd ed. London: RoutledgeFalmer.

Mohan, Giles and Kristian Stokke. (2000) Participatory Development and Empowerment: The Dangers of Localism (pp. 247-68). Third World Quarterly. Vol. 2. No. 21.

Novak and Middleton. (2008) Concept Design Report for Arlington Apartments. [Held at WCC].

Planning Aid England. (2010) Good practice guide to public engagement in development schemes. <http://www.rtpi.org.uk/download/12967/Good-Practice-GuideSpreads.pdf> Accessed 13.01.12.

Reason, Peter and Hillary Bradbury (eds). (2006) Handbook of action research: concise paperback edition. London: Sage Publications.

Riessman, Catherine. (2002) Narrative Analysis (pp. 217-70). In Huberman, Michael and Matthew Miles (eds). The qualitative researcher's companion. London: Sage Publications.

The Salvation Army Social Policy and Parliamentary Unit. (2007) Rebuilding the Kiwi Dream: A proposal for affordable housing in New Zealand. <http://www.salvationarmy.org.nz/uploads/RebuildingTheKiwiDream.pdf> Accessed 02.02.12.

Sanoff, Henry. (2008) Multiple Views of Participatory Design.(pp. 57-69) International Journal of Architectural Research. Vol. 2. Iss. 1.

Sanoff, Henry. (2007) Book Reviews (pp. 173-74). Urban Design International. 
Vol. 12. Iss. 2-3.

Simon, H. A. (1969) The Sciences of the Artificial. Cambridge: M.I.T Press.

UN-Habitat. (1987) Global Report on Human Settlements. Oxford: Oxford University Press.

Viegas, Fernanda, Martin Wattenberg and Jonathan Feinberg. (2009) Participatory Visualization with Wordle. Visualization and Computer Graphics. Vol. 15. No. 6.

Warburton, Diane. (2005) Is participation too expensive? (pp. 316-317). Town and Country Planning. Vol. 10. No. 74.

Wates, Nick. (2000) The Community Planning Handbook: How people can shape their cities, towns and villages in any part of the world. London: Earthscan.

Wates, Nick. (2008) The Community Planning Event Manual: How to use Collaborative Planning and Urban Design Events to Improve your Environment. London: Earthscan.

Wellington City Council. (2010) A Policy for Wellington City Council's Social Housing Service. <www.wellington.govt.nz/plans/policies/housing/pdfs/housing.pdf> Accessed 07.03.12.

Wellington City Council. (2010) Review of the policy for Wellington City Council's Social Housing Service. [Held at WCC].

Wellington City Council. (2000) Framework for the Provision of Housing. [Held at WCC].

Wellington Civic Trust. (2006) Providing for Social Housing in the City Seminar Report on the Seminar. [Held at WCC].

Zeisel, John. (2006) Inquiry by Design: environment/behavior/neuroscience in architecture, interiors, landscape, and planning. New York: W. W. Norton \& Company. 


\section{Appendices}

Appendix A - Interview guide

The semi-structured interview guide I followed:

\section{Semi-structured Interview 1}

[give Info Sheet, run through briefing as per ethics approval]

Name:

Unit:

Age: $\quad<20 \quad 20-30 \quad 30-40 \quad 40-50 \quad 50-60 \quad 60-70 \quad 70-80$

$80+$

Occupation:

Household Structure:

Unit characteristics:

Housing History:

1. If you lived here prior to the $2009 / 10$ upgrade:

- What are the main things you think the upgrade addressed well?

- What are some of the things the upgrade missed?

- $\quad$ Were you involved in any of WCC's tenant engagement prior to the upgrade (Activate sessions, Walk and Talk...)? [How were they? Impact of your involvement on outcomes?] 
2. What are the best qualities of this place?

3. What are worst qualities of this place?

4. Imagine you were back in 2009 , and you were making the final decisions about how Te Ara Hou was to get upgraded. What would you suggest?

5. How well do you think Te Ara Hou fits with its surroundings? [social networks, thoroughfare, playground use...]

Other points? 


\section{Appendix B - Interview responses}

Below are responses to each question coded with letters to ensure anonymity of participants. Not every participant answered every question, therefore there are not necessarily 25 responses to each question documented:

Age:

$<20[\mathrm{P}]$
$<20[\mathrm{M}]$
$<20[\mathrm{H}]$
$30-40[\mathrm{~N}]$
$30-40[\mathrm{C}]$
$50-60[\mathrm{~L}]$
$50-60[\mathrm{~J}]$
$50-60[\mathrm{D}]$
$50-60[\mathrm{~A}]$
$60-70[\mathrm{O}]$

Occupation:

High School Student [M]

High School Student (Y13) [H]

High school student [P]

Full time Student (WelTec) [K]

Invalid [L]

Beneficiary [J]

Nurse Aid [O]

Work at hospital $[\mathrm{A}]$

Taxi driver [B]

Housewife + work at supermar-

ket part-time $[\mathrm{Q}]$

Housewife [G]

Housewife [F]

Housewife $[\mathrm{C}]$

Housewife [N]

Unemployed [D]
Household Structure:

Participant and 2 children [Q]

Mum, Dad and 5 kids [P]

Participant, husband and 3 daughters [O]

Participant, brother and 2 children [N]

Mum and participant [M]

Lives alone [L]

Participant, sister and son $[\mathrm{K}]$

Lives alone [J]

Parents and 2 kids (her and sister) [l]

Parents and participant. [H]

Husband, wife and 2 children [G]

Mother and 7 children [F]

Lives alone [D]

Mother and 1 Son [C]

Husband, Wife and 2 grandchildren [B]

Husband, Wife and 3 children $[\mathrm{A}]$

\section{Unit characteristics}

Bedsit [L]

Bedsit [J]

Bedsit [D]

2 bedroom [H]

2 bedroom [Q]

2 bedroom [N]

2 bedroom [M]

2 bedroom [K]

2 bedroom [G]

2 bedroom [C]

3 bedroom [O]

3 bedroom [A]

4 bedroom [P]

6 bedroom [F] 


\section{Housing History:}

Been here 1.5 years [Q]

Been here 17 years - grew up here. [P]

Been here 30 years - been here longest out of all tenants [0]

Been at TEA almost 2 years, Newtown Park before that. [N]

Been in this unit at TEA most of my life (13 years) [M]

Been at TEA 18 years, in the same unit [L]

Been here 5 years $[\mathrm{K}]$

Moved here 3.5 years prior to upgrade [5 years ago] [J]

Te Ara Hou has been home for 16 years. [l]

Been here 2 years. [H]

Been here 1.5 years. Arrived in NZ 2008 and lived in private housing before moving in here. [G]

4 years at Te Ara Hou [F]

At Te Ara Hou since 1994. Shifted units in 2004 [E]

Moved to Te Ara Hou in 2005. [D]

Been at Te Ara Hou since 1995 (16 years) [C]

Been at Te Ara Hou 14 years. [C]

Been at Te Ara Hou 20 years. Was at a different flat in TEA prior to the upgrade. [A]

1. If you lived here prior to the $2009 / 10$ upgrade:

What are the main things you think the upgrade addressed well?

- New carpet and better walls are good. Playground is good. Better windows now. $[\mathrm{P}]$

- [jumped to negatives first, then said:] They made the outside pretty (so it all looks good from the outside and to the public). [0]

- Wasn't here before upgrade [N]

- It looks much better now. Before, plants all trampled on. It's much brighter now. Unit is a lot warmer, too. [M]

- Landscaping. It's nice walking around the grounds now, seeing all the flowers in spring, the trees, shrubs... Also easier to keep the flat warm. Condensation cured. Better ventilation and insulation. [L]

- 1. The community. Since the upgrade, everyone is just more humble now. There's way less aggression. People are proud to be living in these flash apartments. Everyone is just positive now - people are actually proud to invite their friends over now, "you should see my place..." No urine smell in the stairs 
etc. Kids friends ask them "are you rich or something?!"

2. Insulation and double glazing made a big difference. [K]

- 'It's a total disappointment' [see below]. Double glazing and insulation are good (notice noise from downstairs a lot now - perhaps simply because I can't hear any road noise or anything else from outside!). Site improvements really good, outdoor spaces great for ground floor units. Not much they changed or could have changed for us up here on 4th floor. [J]

- There are no more rats! We'd see rats through here all the time. Bathrooms better. Lawns look way better. [l]

- Moved in post-upgrade. Had seen the place before though, and it has improved a lot. [G]

- Playground has improved a lot. Also painting (and general improvements) inside units. [F]

- Units much warmer (only needed heater on 3 times this year). This main improvement. Complex much cleaner, it's just tidier. Council cleaning up rubbish every few days, much better. New rubbish system much better - no smell. Privacy much better now with fences around outdoor areas on ground floor (space for kids to play safely separated from cars). [E]

- Insulation and double glazing made a huge difference. Entranceways have been improved. [D]

- A lot has improved - the bathrooms are better, better ventilation, heaps of things. The playground is good for kids. [C]

- For the moment, all is going well. [B]

- Happy with everything. Likes the private outdoor area. $[\mathrm{A}]$

What are some of the things the upgrade missed?

- No negatives [P]

- [Participant wasn't happy with result of the work]: Generally bad workmanship ('Fletchers had their trainees on this job'). Handrails in bathroom now on opposite side of room from sink (becomes a safety issue - 'I've slipped before, it's an OSH issue'). Draught comes through windows even when they're shut - small holes for ventilation too large. Cheap construction, weak walls etc.

- They shifted the towel rails in the bathroom away from the sink - have to go to other side of bathroom to dry your hands now. [M]

- Don't know... Some cheap materials used, but I guess they had to keep costs to a minimum. [L]

- Doors rattle in the wind. Toilet roll holder has broken off. [K] 
- Bad workmanship (I am a bit of a perfectionist). 'It's worse than before' in some respects. Showerhead is leaking into the wall rotting the lining on other side wasn't like this before upgrade. Deck doesn't drain - not sloped enough. Bad drainage in some places around site. Generally, a bit more care was required. "I thought Fletchers was a reputable company..." [J]

- The unit still gets cold. [l]

- No, all happy. [F]

- New washing lines are a bit weak in the wind. Generally though - it's just way better what we've got now. Some bad workmanship (toilet roll holder falling off). [E]

- Landscaping has been unsuccessful - wind is blowing trees over. Many loose flashings weren't fixed. Painting workmanship has been a bit poor. We've lost pedestrian path out the north-east corner of the site. [D]

- $\quad$ No great problems. [C]

- $\quad$ All is OK. [B]

- $\quad$ Nothing really - pretty happy. [A]

Were you involved in any of WCC's tenant engagement prior to the upgrade (Activate sessions, Walk and Talk...)? [How were they? Impact of your involvement on outcomes?]

- Didn't go - Mum did. [P]

- No. I knew they were doing it, 'but l'd heard it all before.' Skeptical about Council's intentions. Daughters went along though [l asked them how it was] 'it was good'.

- No, I didn't go. [M]

- Yes, I went along. There was some tenant input, Council listened. I had contributions around landscaping. I also suggested railings around the balconies for safety. [L]

- Yes, they were really informative. We had discussions about people's ideas for the upgrade - one of mine was about the small bathrooms. We also asked for lifts for the elderly people who are often in the bedsits on top floor and struggle with stairs. Listing all the points for Council to consider in the upgrade was fine, people gave a lot, but behind closed doors there was no confidence in Council. Tenants have a real lack of confidence. For years people would voice their concerns and nothing would get done, so there is no trust. [Participant has good relationships with people 'higher up' at Council now so is able to get things done. Tenants come to participant now to get responses to mainte- 
nance concerns when normal channels don't work]. [K]

- Wasn't involved. Knew of them, just didn't know what to suggest and thought they were more for the families, really. [J]

- Yes, went to Activates [they identified almost everyone in the photos I had]. They were all good. Felt like we impacted everything. [F]

- Yes, went to an Activate. It was good Council listened to what tenants wanted. I wasn't too worried about designs. It's really good that they came in to ask feedback, but I was quite happy. 'I just agreed with whatever they suggested' [E]

- Yes, I recall some people being very vocal about paint colours. Council made a big show of looking like they're listening. They did consult quite a bit, I'm not convinced that they followed through with everything though. They made a lot of promises. [D]

- I attended one Activate. Some good suggestions that tenants contributed in the sessions were that they required storage areas for prams and they didn't want the rubbish chutes. [C]

- Yes, I attended Activates. They were useful. [Asked: Did you feel like your contribution made a difference?]

- $\quad$ Yes. $[\mathrm{B}]$

\section{What are the best qualities of this place?}

- Playground very good [Q]

- The people - they get along really well. Respect between cultures is good. Activities put on for kids are great (we did go-karting once, visited Parliament) [P]

- We're on the edge here (against Constable St), we can mind out own business. More private. I don't see much of what goes on in the rest of the complex anymore. We were in Block $\mathrm{C}$ before, it was much more public. [0]

- Children's playground is great. 'Not too bad' people. Neighbour one side very good. The other side - not good. Leave rubbish outside, no respect for neighbours. Island people don't have respect. [N]

- The people. People are really nice here. [M]

- Location - it's close to most things I need. It's also nice being in a north facing unit - gets good sun. [L]

- A lot warmer in winter. The heater installed in upgrade only takes one hour to heat the whole place and it doesn't chew much power. [K]

- Surroundings, location. Feels safe. Used to be dodgy with bad lighting etc, this is much better now. Good placement of people on floors too - council seem to 
have got that right now (of similar nature to neighbours). [J]

- The people [grew up here, very attached to this place]. New privacy for outdoor areas off ground floor units good. [I]

- Convenient, good location. Balconies great for sun. [H]

- Everything is close - kindy, school, hospital. Good location. Bus stop close. [G]

- Playground [daughter's point]. Lots of sun - nice sunny. [F]

- Great location, handy to everything (our Samoan church on Owen St, the hospital as our son is unwell). [E]

- The family aspect is good here. Impact neighbours have on life is really high, so it's good that neighbours are generally good here. Somali refugee families are very pleasant, less social. The playground is well used. It's a great location, everything is handy. [D]

- The upgrade changed a lot. It's a lot better than before. I never had a problem before though... [C]

- Everything is OK - close to shopping, hospital... everything is close. [B]

- Location is good (close to work, church, schools), as we don't have a car. [A]

\section{What are worst qualities of this place?}

- No nothing, it's nice! [Q]

- There are probably some, but l'm not sure. [P]

- I've seen bullets go through windows here. People kicking in doors. I've seen people jump off balconies. I've seen knives, stabbings. This mainly comes from patients - often schizophrenic - and often visitors, not tenants. Some of the best times, though, have been the school holiday programmes. One year a mayor donated a pool table and other things for the programme. Mums took turns on days, then at the end of the week we had a big meal, all brought a plate. These have been the best times. [0]

- This sitting room is too small when you've got kids. Also kitchen too small, and toilet too small. Not all two bedroom units are the same - these on 3rd floor much smaller. That's my only complaint though. [N]

- The park looks a bit childish.. It's for younger kids. We [participant is 13yo] use it too, but it's a bit childish. I practice netball on the basketball court sometimes, and it's a bit childish with the giraffe on the pole. [M]

- Over the years some of the neighbours have been a bit rowdy. Noise issue. Not currently though. We had a drug dealer in the building a while back. Things have been pretty settled and quiet the last year or two. [L] 
- Communication with new tenants is hard. We want to get to know everyone who lives here, but they often keep to themselves. [K]

- That poor workmanship. [J]

- Nothing. It's seriously better than before. This question is probably better to ask new people who didn't know it before. [l]

- It can get too hot in summer and too cold in winter inside the units. [H]

- I guess the house is a bit small, but alright. Kid's room is small - not enough space for two beds (baby sleeps on bed, older boy has to sleep on a matress). [G]

- No, it's all fine! [F]

- Noise from the unit above is a problem after $10 \mathrm{pm}$ - yelling, kids playing. Relatives and other visitors using car park is an issue. There's not enough car parking. There aren't enough washing machines and driers. [E]

- Some neighbours. You're always going to get some bad neighbours in council flats, but it's better here than other complexes (lived at Arlington before here - very bad experiences with neighbours there). [D]

- Some people struggle with language barriers etc. [C]

- Nothing. [B]

- Hearing people's voices (from outside). Also hearing noise between apartments in the middle of the night. Another problem is kids (not from TEA) making trouble in the playground. $[\mathrm{A}]$

\section{Imagine you were back in 2009 , and you were making the final decisions} about how Te Ara Hou was to get upgraded. What would you suggest?

- Don't know. [Q]

- Earthquake strengthening [I explained that Te Ara Hou didn't require as much attention for earthquake strengthening as many of the other complexes have]. Also, l'd extend the buildings for more rooms. [P]

- Proper walls, better quality GIB. Better quality finishes and fittings. [O]

- We could have a field for soccer and stuff - had one before upgrade in that picnic tables area (as there weren't private outdoor areas and plantings). Also, a bigger court for basketball would be good. [M]

- I'm glad l've got a garden box now. I've got one of 9 in the community garden. It had to be gated and locked as some parts of the garden were getting trashed. I had my own planter box on the balcony here before the upgrade - after the upgrade Council said I wasn't allowed to keep it. It's fine though. [Participant showed me his many small pot plants (cacti etc) on the balcony - 
he sells them at his stall at a monthly market]. [L]

- Those trees that aren't taking. Kids (often from outside the complex - relatives) often climb them and snap them. If we ever see anyone harming them we'll put a stop to it, but keeps happening. Need protection for those - metal frame bolted to ground. [K]

- Don't really know... [J]

- Get us a new stove (ours keeps breaking down). And a new bathtub (we've still got old one - hard to clean). A volleyball net on the lawn outside would be cool. [l]

- I would suggest a playing area for older people - teenagers (to play bowls or something like that, more appropriate for that age group). $[\mathrm{H}]$

- Nothing. [G]

- Nothing. [B]

- Don't know. $[\mathrm{A}]$

5. How well do you think Te Ara Hou fits with its surroundings? [social networks, thoroughfare, playground use...]

- Community, friendships are mostly in Te Ara Hou flats. [Q]

- Community, friendships are mostly in Te Ara Hou flats. [P]

- We have many friends here within Te Ara Hou. [M]

- Most of our family's social networks are outside Te Ara Hou - we don't know that many people here. [H]

- Especially in summer, lots of people come here to play. Also, the hall is used a lot. [C]

- Most friends in Te Ara Hou - some outside the complex too. There's a good community here. [B]

\section{Other points?}

- Not being racist or anything, but l've noticed kiwis getting pushed aside out of units for refugees - Somalis. When we got asked to leave our old 6 bedroom unit, it was a Somali family who moved in there. [0]

- Us old tenants tend to stick together, we're one big family. There's a bit of division between old tenants and new tenants. [0]

- Chatted at length about history of unrest in Somalia, their family back home stuck in conflict. Came here as a refugee in the 90's. 'Very hard to get to New Zealand.' [Asked how they managed to come here] 'God allowed it'. [N] 
- [We discussed tranferring to a unit on lower floors] - it's very hard to transfer now with the upgrade as there is a shortage of units as people are being relocated. Would ideally be on the ground floor - no stairs to walk up with children and shopping! [N]

- Had a discussion about views on temporary/perminant nature of council housing. Concern at the moment as some tenants around city only being given short leases (during upgrade). Participant's view is if people want to stay long term, they should be able to. Hasn't heard of any cases of people having to leave because of rising income. Knows some cases of tenants having to leave as they took a job at Council (perhaps this was due to higher income). Once you're perminant, you're perminant. There's no pressure to leave. Council doesn't push at all, so long as you pay your rent etc. And people are really happy here, it's home and people don't want to leave. [K]

- Getting rid of gang problems here has made a big difference as well as improvements to the buildings. Used to be clashes between Darksiders (connected to Black Power) and Full Blooded Islanders (FBI). Still some gang presence sometimes, visiting relatives in units etc but much less negative impact from gangs now. [K]

- Spoke about the success of the new mosaic mural at the entrance to Block C. Participant was involved in this. This project was great in bringing tenants together. [K]

- Participant had a lot to do with leading the park upgrade which happened at the same time as the upgrade. Because kids had a lot to do with creating the park, they work hard to protect it. We see kids asking members of the public to pick up rubbish they dropped now - they don't want it ruined. Less of an issue with people coming to the park to drink now too, participant usually leads the charge getting rid of them too. [Seems classic case of people participating in the creation of something then working a lot harder to protect it than they would have otherwise. A key positive outcome] [K]

- Communal vege garden works well, only for limited number of people though. Discussed possibility of vege gardens on balconies - Council don't allow gardens or even pot plants on balconies. [K]

- We spoke at length about Wellington buildings, our favourite Art Deco buildings, etc. Great views out of top floor unit - pointing out various buildings in view. [J]

- Lived at Newtown Park while Te Ara Hou was being upgraded - like that unit despite the complex's bad publicity of late. Full height glazing, that was a $1 \mathrm{bdr}$ unit (not a bedsit) too. [J] 
- New housing would be great - great idea! They could put it up overe these carparks (there are so few anyway). Or over the other car parking area. This could be more Council housing for people on the waiting list, or maybe for people to move out of Newtown Park (so dangerous there). OR, it could be new housing for people to buy - because people here get really dependant and need something to move on into. OR it could even be private housing, that'd be cool. Council could make some money. OR they could even sell off some of these (existing) units - l'd buy this unit! l'd buy the whole block if I was rich! [l]

- On availability for workshops - might be available, wants reminded. Children will definitely come! [F]

- Willing to do a workshop - 4.30pm weekday should suit - 'come back and remind me.' [G]

- Might be a but busy to do a workshop (even in school holidays, going in every day to catch up on work). [H]

- New housing on the site is a good idea... More people would be good, and fine for them to be non-council residents. [H]

- [Asked for view on new private housing being developed] If new housing was developed here on the site, I wouldn't want to move into it - I'm happy in this unit, I like my neighbours etc. I don't want to move into private housing again as everything is good here - when something is wrong, Council always fix it etc. [G]

- [Asked for view on new private housing being developed] - yeah, if you can do it, it's a good idea. Already lots of people here though, don't want many more. There are some spaces it could work, but already quite crowded. [F]

- The communal vege garden - I did sign up, so I'm supposed to use it. I don't have time though. Also, I don't quite trust other tenants using it, they may spoil or contaminate my vegetables. [E]

- I don't use the bike storage room downstairs for our pram as some people put their motorbikes in there so it would make my pram smell like petrol which isn't good for baby. l'd rather store pram inside. [E]

- We need a gate on the basketball court area to separate it from the road for child safety. This was better before the upgrade. [E]

- [Asked for view on non-council tenants living in the complex] - no problem with this. It's already a diverse community. It's good to have different cultures together. Biggest concern around introduction of non-Council tenants would be lack of carparks. Also, the place might get messier, dirtier. But more people (and more friends) is a good thing! [E] 
- Frankly, for all the niggly concerns we've talked about, I'm grateful that Council provides this low-cost housing. And actually, if they had to respond to all these picky little things (flashings etc), perhaps it wouldn't be so cheap. I just hope Council continues to provide housing. [D]

- Most of my friends are in Te Ara Hou. There's a good community here. A new family just moved into our block. [Seemed to know a lot of others in the complex - could tell me where all the Samoans live. Very aware of tenant comings and goings]. $[\mathrm{A}]$ 


\section{Appendix C - Creating word clouds}

Here the process for generating word clouds is elaborated upon. Word clouds are generated automatically at www.wordle.net. Here, the question '2. What are the best qualities of this place?' is used as an example.

\section{FULL TEXT RESPONSES:}

- Playground very good [Q]

- The people - they get along really well. Respect between cultures is good. Activities put on for kids are great (we did go-karting once, visited Parliament) [P]

- We're on the edge here (against Constable St), we can mind out own business. More private. I don't see much of what goes on in the rest of the complex anymore. We were in Block $\mathrm{C}$ before, it was much more public. [0]

- Children's playground is great. 'Not too bad' people. Neighbour one side very good. The other side - not good. Leave rubbish outside, no respect for neighbours. Island people don't have respect. [N]

- The people. People are really nice here. [M]

- Location - it's close to most things I need. It's also nice being in a north facing unit - gets good sun. [L]

- A lot warmer in winter. The heater installed in upgrade only takes one hour to heat the whole place and it doesn't chew much power. [K]

- Surroundings, location. Feels safe. Used to be dodgy with bad lighting etc, this is much better now. Good placement of people on floors too - council seem to have got that right now (of similar nature to neighbours). [J]

- The people [grew up here, very attached to this place]. New privacy for outdoor areas off ground floor units good. [l]

- Convenient, good location. Balconies great for sun. [H]

- Everything is close - kindy, school, hospital. Good location. Bus stop close. [G]

- Playground [daughter's point]. Lots of sun - nice sunny. [F]

- Great location, handy to everything (our Samoan church on Owen St, the hospital as our son is unwell). [E]

- The family aspect is good here. Impact neighbours have on life is really high, so it's good that neighbours are generally good here. Somali refugee families are very pleasant, less social. The playground is well used. It's a great location, everything is handy. [D]

- The upgrade changed a lot. It's a lot better than before. I never had a problem 
before though... [C]

- Everything is OK - close to shopping, hospital... everything is close. [B]

- Location is good (close to work, church, schools), as we don't have a car. [A]

These full-text responses fed into the generator at www.wordle.net create this word cloud:

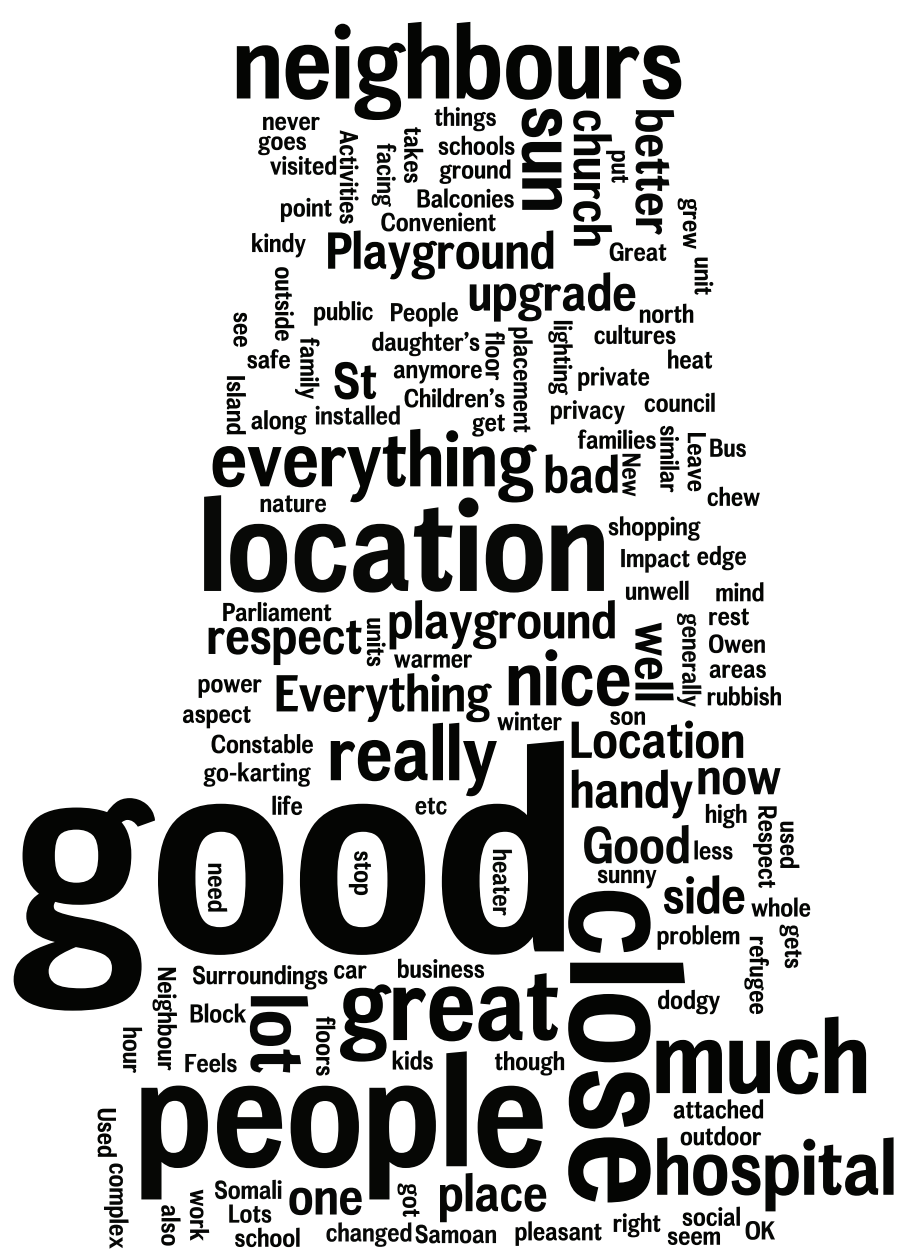

It became clear that the term 'good' is not particularly informative in communicating interview responses in graphic form. It is for this reason that I manually aggregated responses from their full-text form, so that 'Wordle' could generate a more insightful graphic. For example, "Convenient, good location. Balconies great for sun" was aggregated into "location" and "sunny unit". 
AGGREGATED RESPONSES:

location

sunny unit

location

sunny unit

location

sunny unit

location

location

private outdoor area

location

location

warm unit

upgrade improved a lot

private unit

family atmosphere

the people

the people

good neighbours

the people

good neighbours

respect between cultures

playground

playground

activities for kids

playground

These responses generated the above graphic. One can see that as 'location' was mentioned seven times, it becomes represented by the largest font size.

'Respect between cultures' is small in the graphic, as it was only mentioned once in the responses.

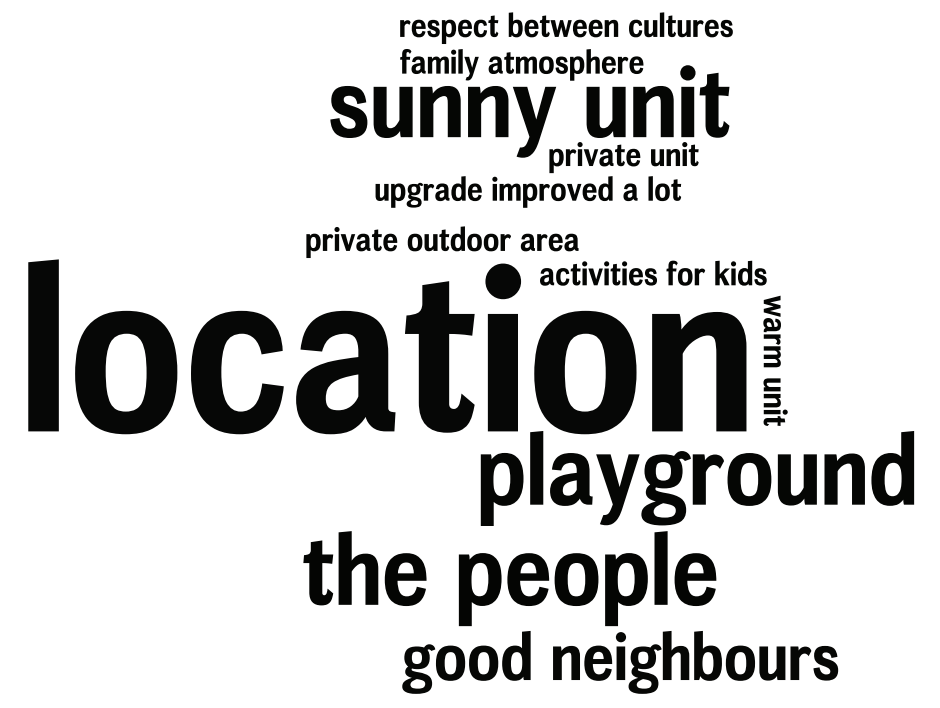




\section{Appendix D - Medium density housing precedents}

1.

Scheme: Beaumont Quarter (Masterplan)

Architect: Studio Pacific Architects

NZIA Award: 2010; National Award; Urban Design (among others)

\section{Lessons for the Te Ara Hou Infill Development:}

- Relevant for considering threshold from private unit to public realm. The layering of space from public to private at Beaumont Quarter is exemplary.

- Significant public landscaped areas, preserved mature trees where possible.

2.

Scheme: Beaumont Quarter (Stage One Housing)

Architect: Studio Pacific Architects

NZIA Award: 2003; Local Award, Multiple Housing

\section{Lessons for the Te Ara Hou Infill Development:}

- Mix of cladding materials and variation in building designs offers variation.

- Heritage buildings conserved and integrated into new development to good effect.

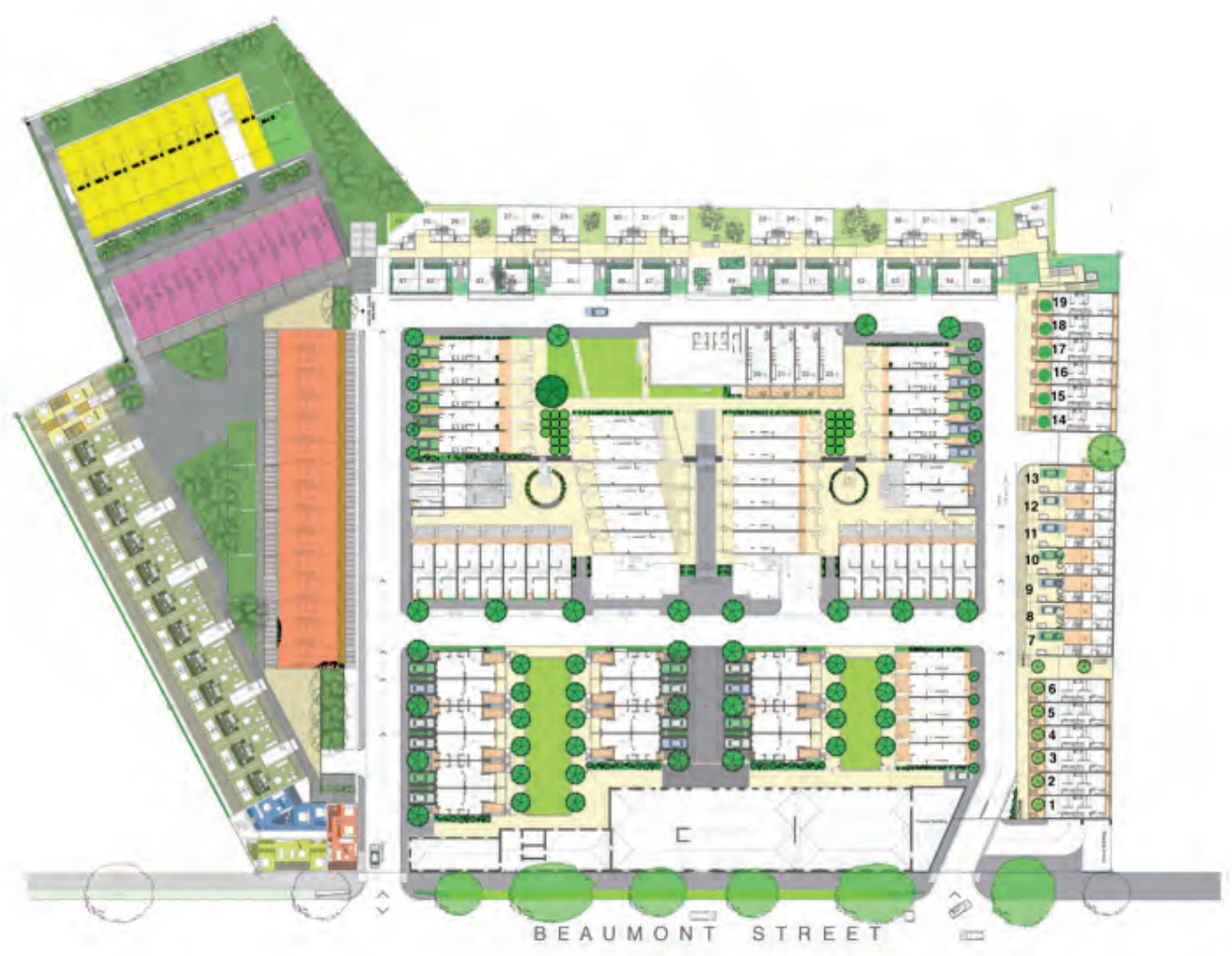

Figure D1 - Beaumont Quarter Masterplan Photo used with permission from architects 
Figure D2 - Beaumont Quarter: Repeated elements interrupted with changes in material palette

Photo used with permission from architects

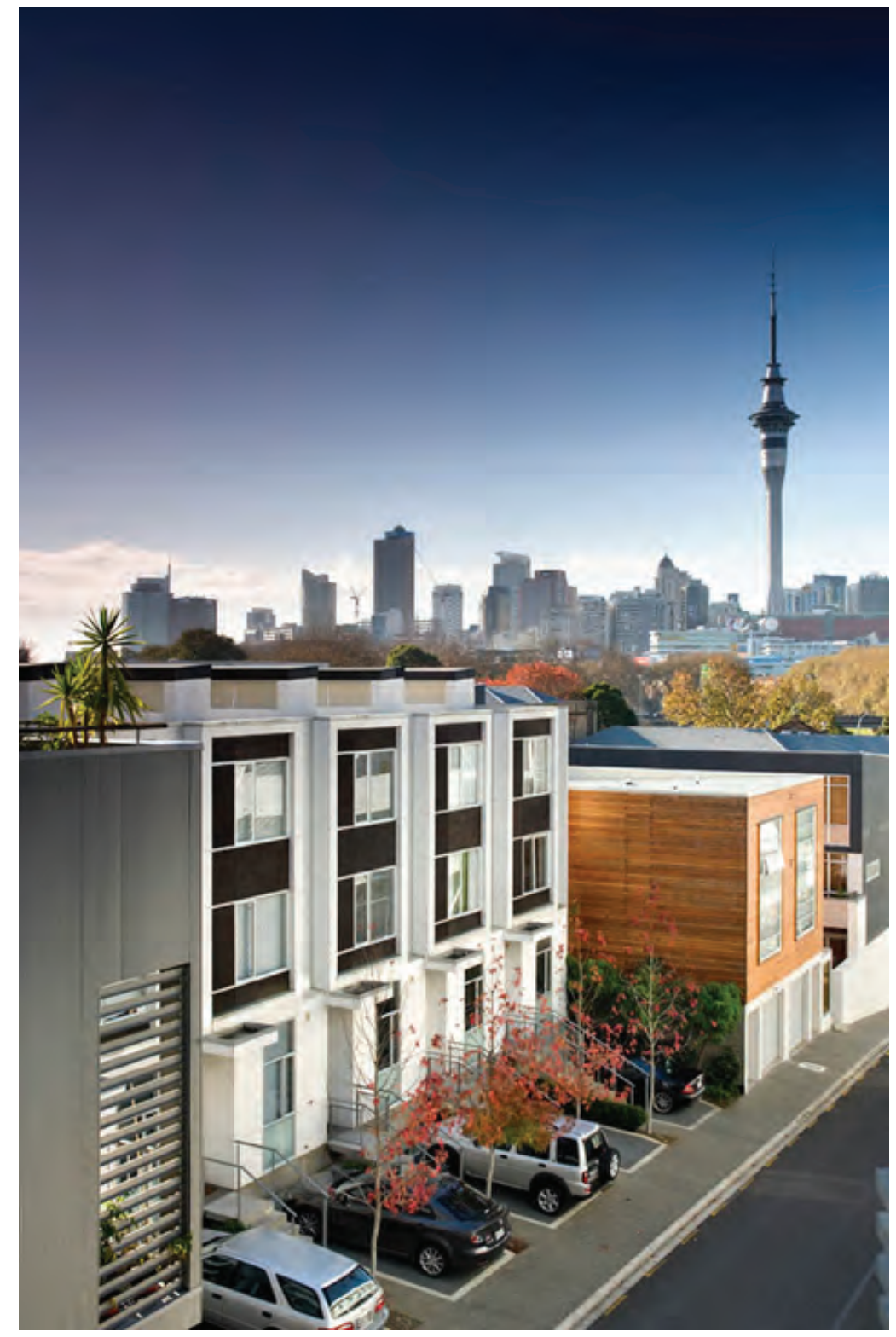



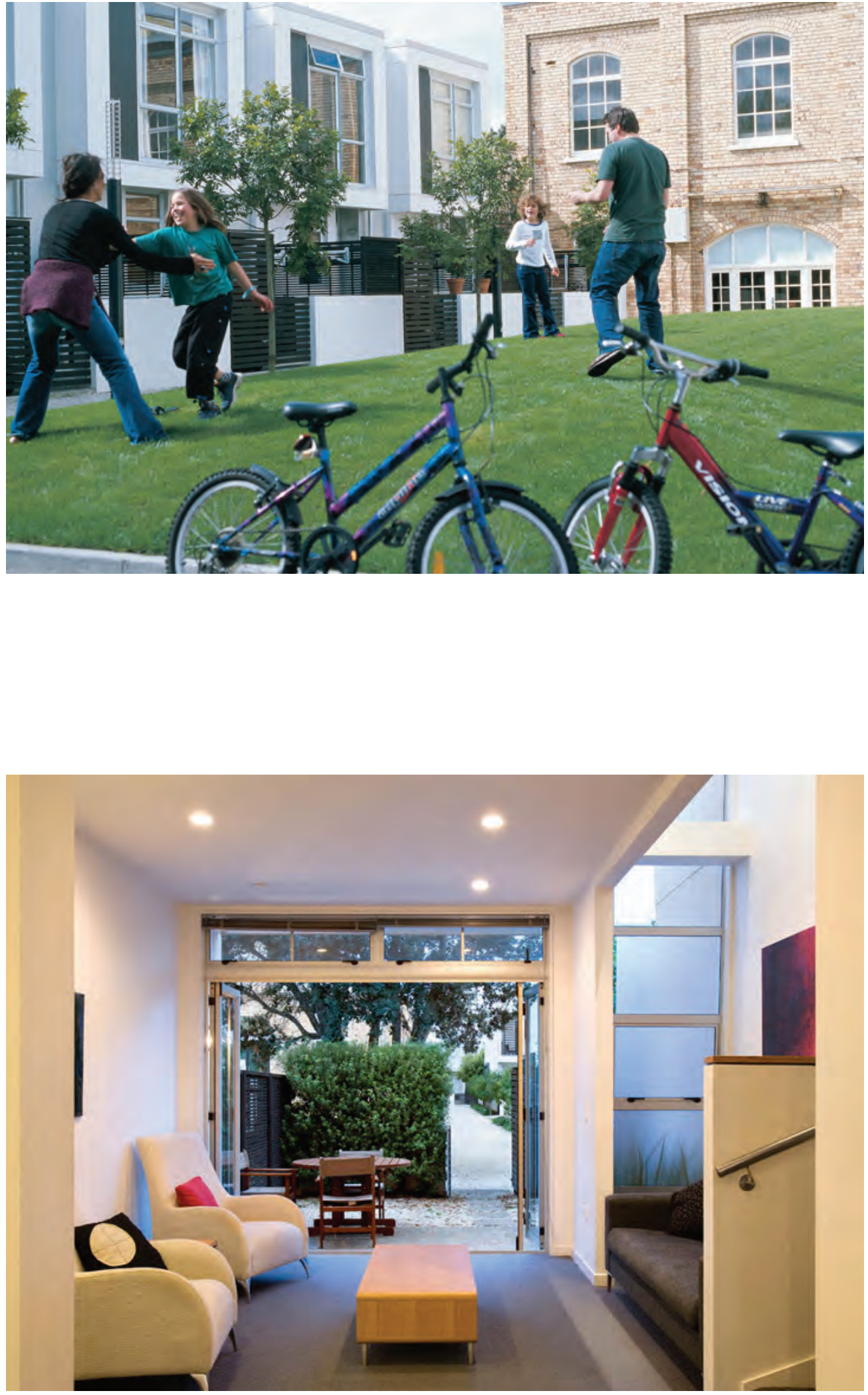

Figure D3 - Beaumont Quarter: Good gradation of outdoor spaces from private fenced areas to public lawns.

Photo used with permission from architects
Figure D4 - Beaumont Quarter: Some private outdoor space with more public areas beyond.

Photo used with permission from architects 
3.

Scheme: Thackeray Street Apartments, Hamilton

Architect: Mercer \& Mercer Architects Ltd

NZIA Award: 2009; National Award; Residential Architecture - Multiple Housing Lessons for the Te Ara Hou Infill Development:

- More inward-looking approach to outdoor space and outlook

- Had challenge of facing south to the street and north to sun, result being that outdoor spaces face away from the street

- Generously proportioned spaces

Figure D5 - Thackeray Street Apartments: Deep balconies make interior spaces more private Photo used with permission from architects

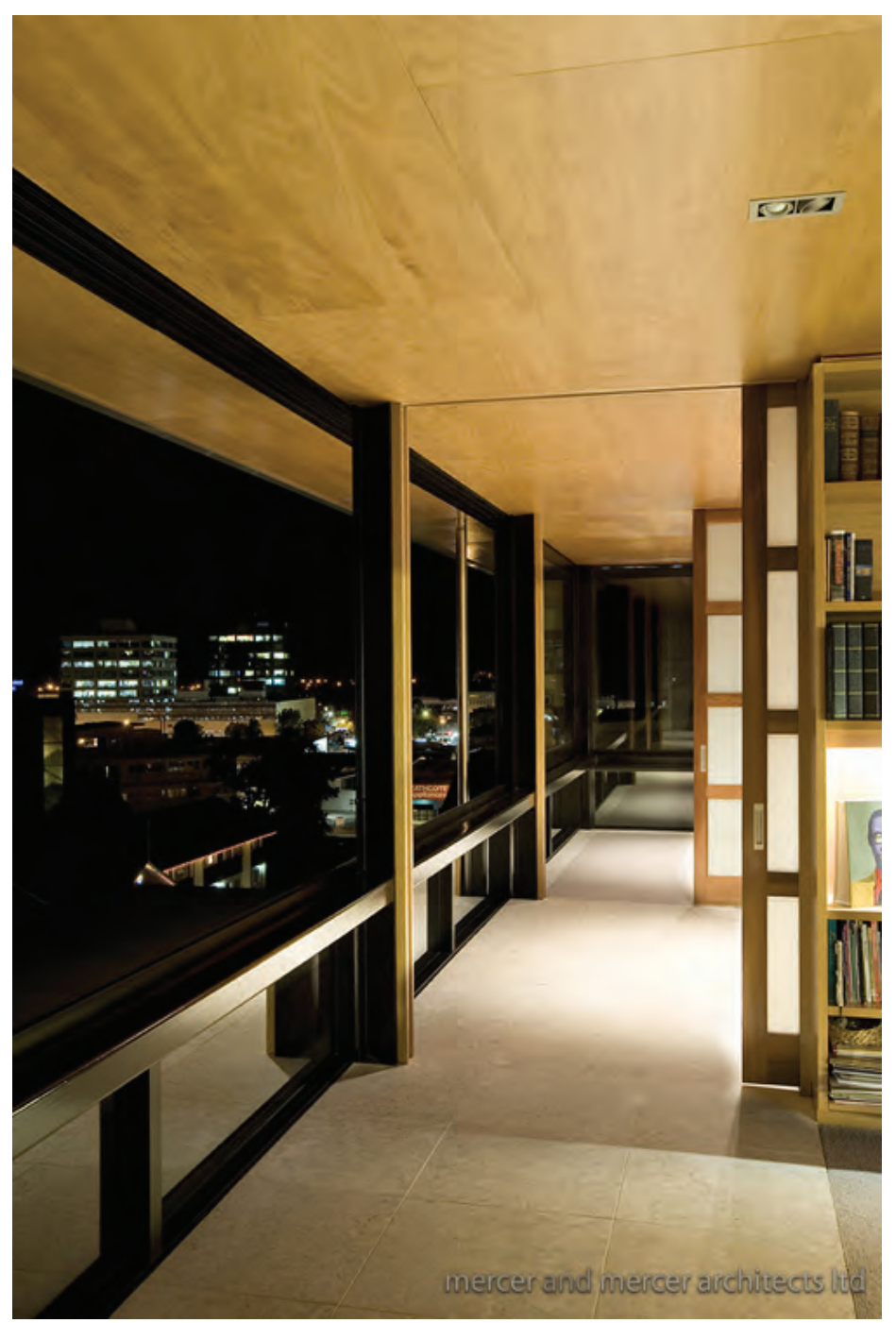




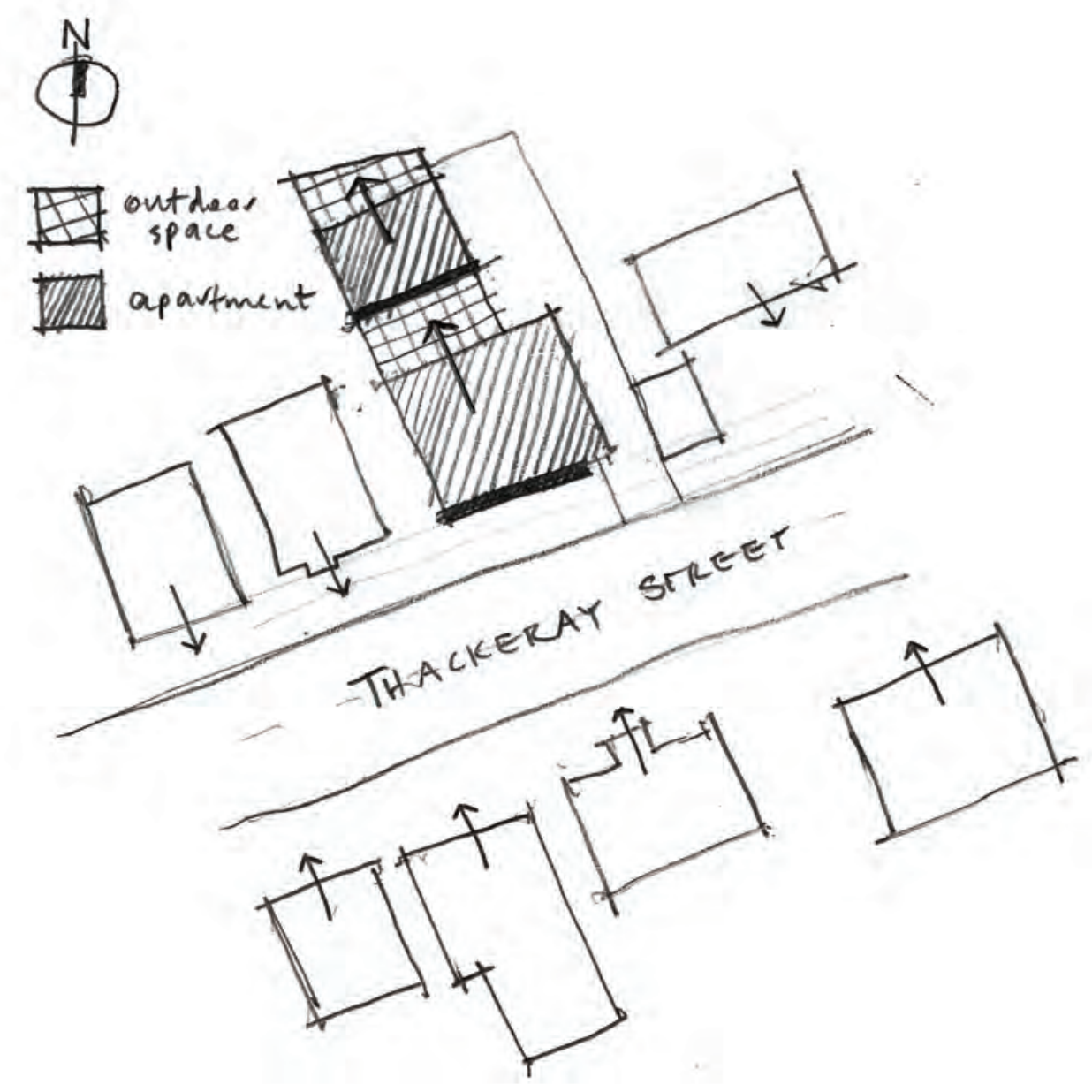

Figure D6 - Thack-

eray Street Apartments: Sketched site plan (by author)

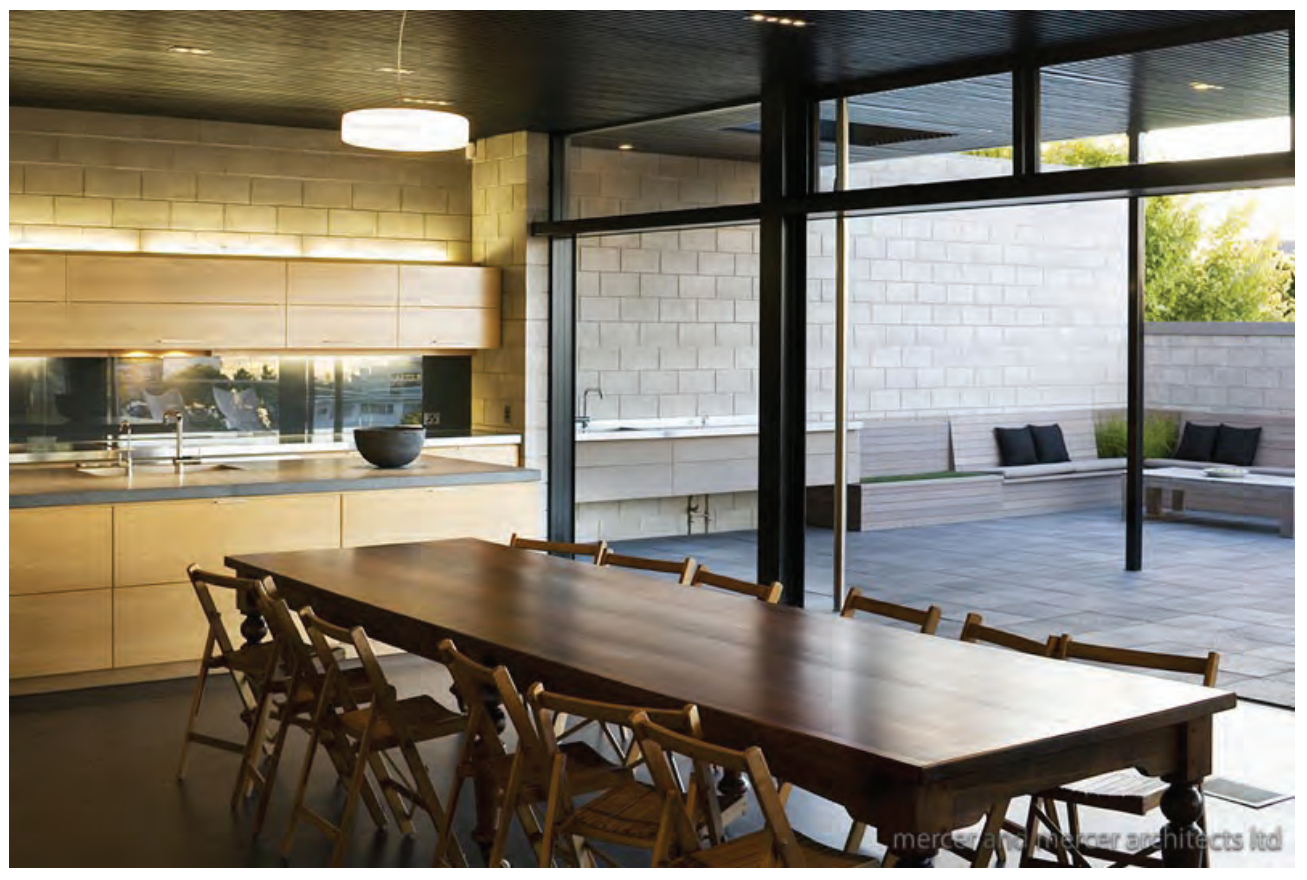

Figure D7 - Thackeray Street Apartments: "The density may be compact but the spaces are generous, light-filled and welcoming" (award citation).

Photo used with permission from architects 
4.

Scheme: St Mary's Court

Architect: Peter Beaven Architect

NZIA Award: 2007; National Award; Multiple Housing

\section{Lessons for the Te Ara Hou Infill Development:}

- All units have openings on both sides. Beaven says "you've got to have through-ventilation, which is the most critical thing in high density housing" (Walsh 138).

- Asked if people use the public space, Beaven replied, "No, they sit in their own places" (flats have either a private courtyard or a balcony). "I don't think that in high density housing like this you can actually make communal areas, because people by their nature want their own spaces" (Walsh 142).

- Material/colour palette of new intervention relates directly to old chapel.

Figure D8 - St Mary's Court - Respectful reference to existing built context in colour and form. Photograph by Patrick Reynolds, in 'Home Work: Leading New Zealand architects own houses' $p 133$ (used with permission)

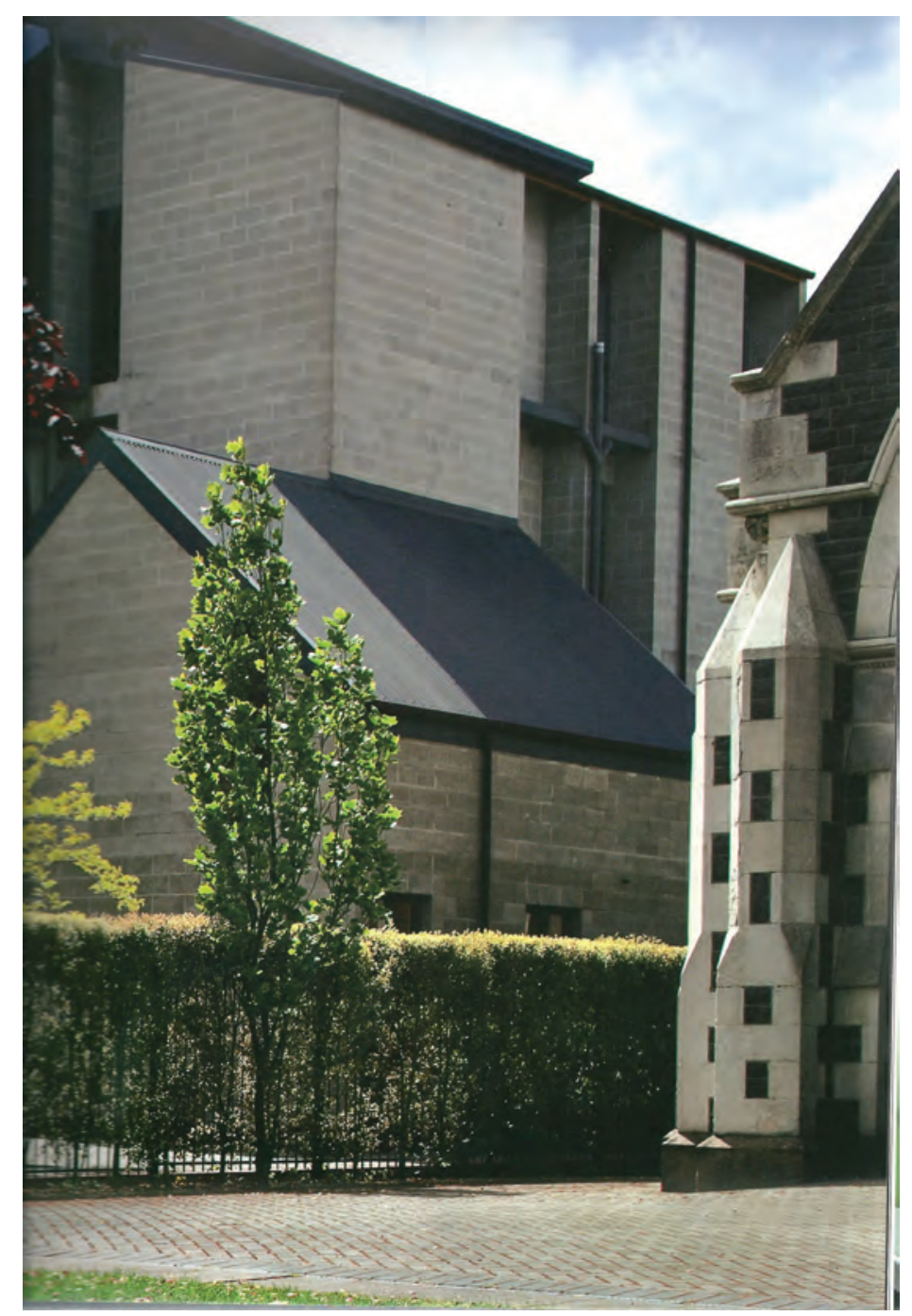



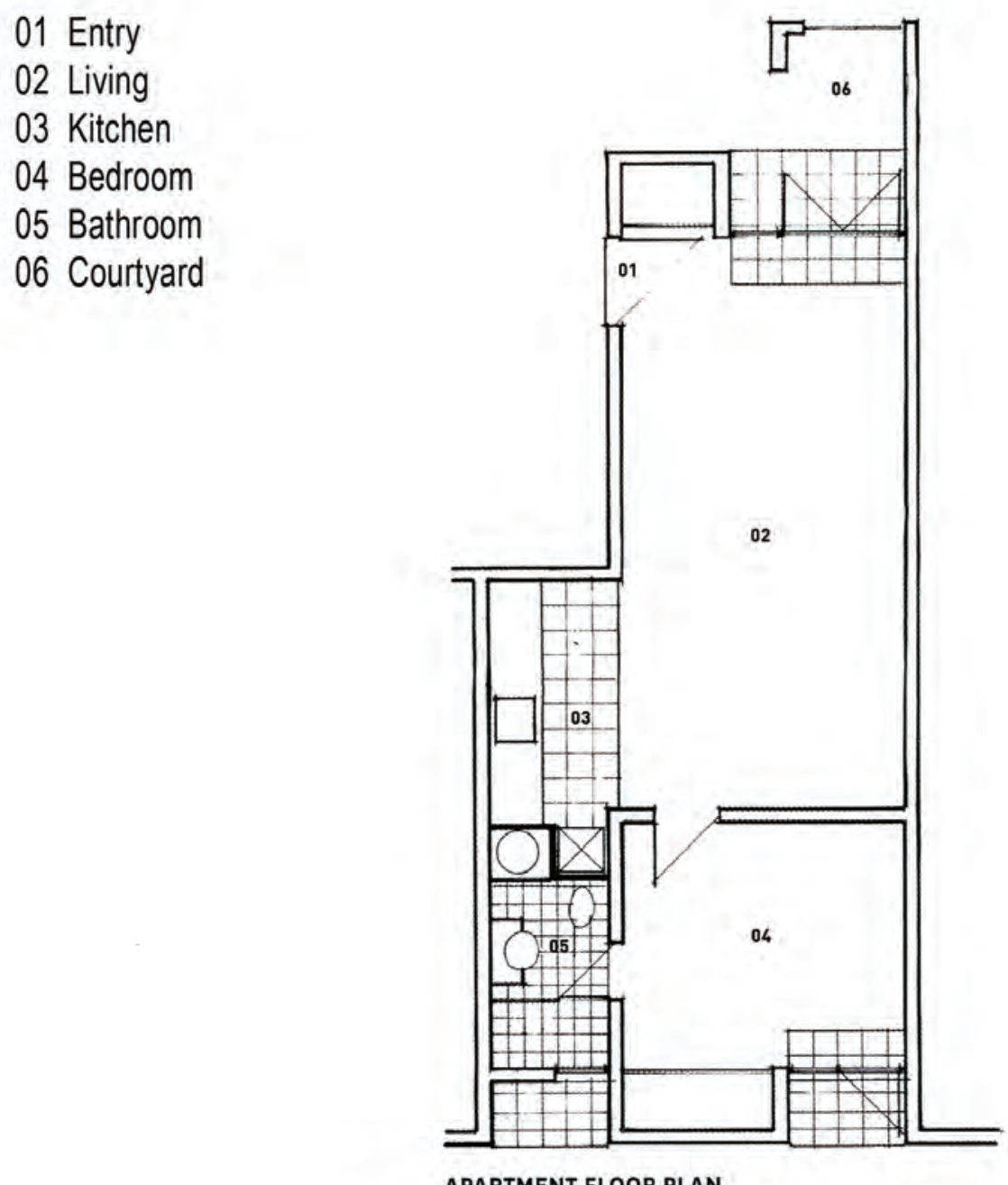

$\int^{\stackrel{2}{2}}$

APARTMENT FLOOR PLAN

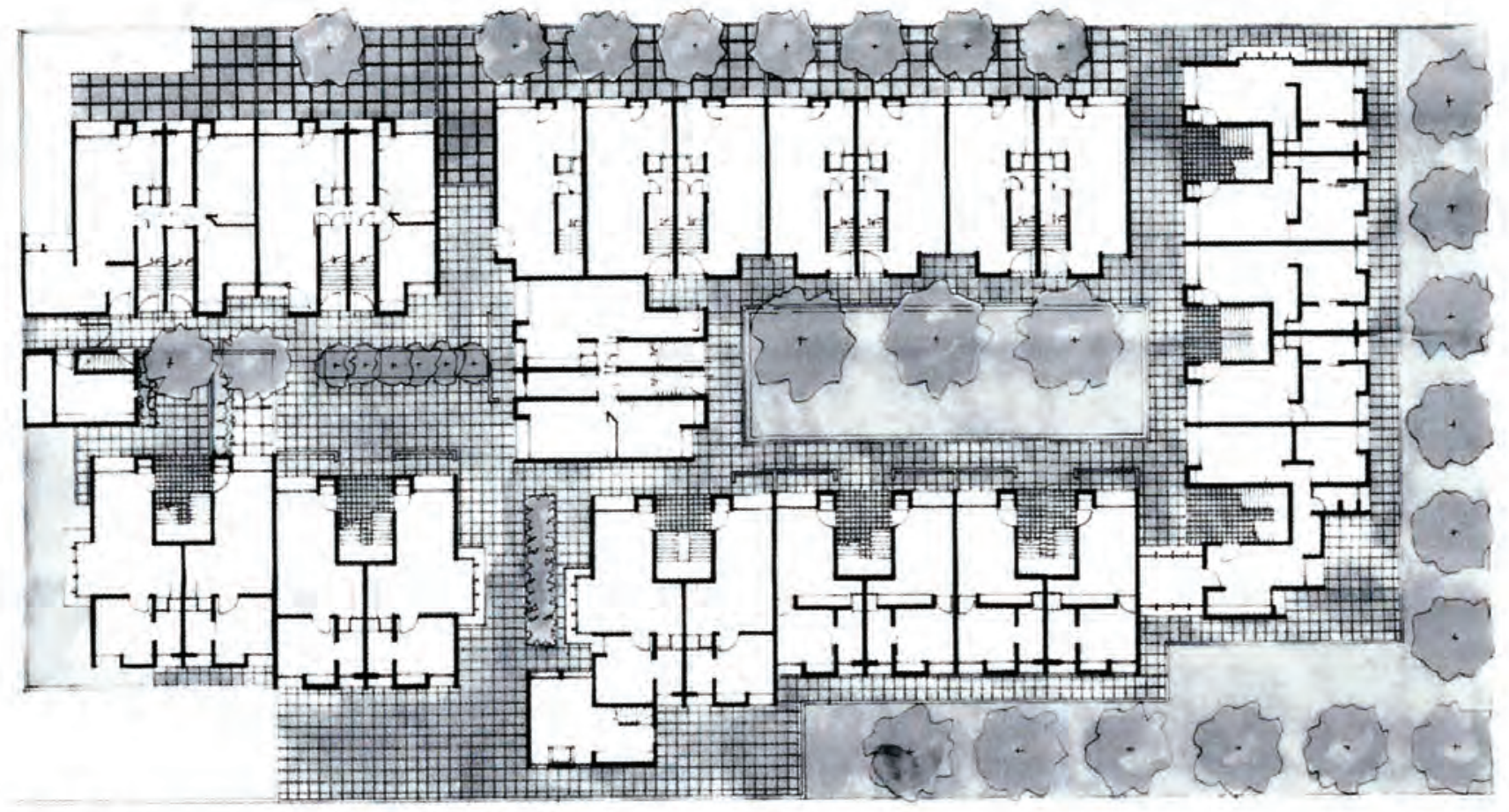

SITE PLAN

Figure D9 - St Mary's Court - Site Plan and Typical One Bedroom Floor Plan.

Plans by Peter Beaven, in 'Home Work: Leading New Zealand architects' own houses' p145 (used with permission) 
Figure D10 - St Mary's Court - Communal areas: used more for walking through and looking onto than used communally Photograph by Patrick Reynolds, in 'Home Work: Leading New Zealand architects' own houses' 136 (used with permission)

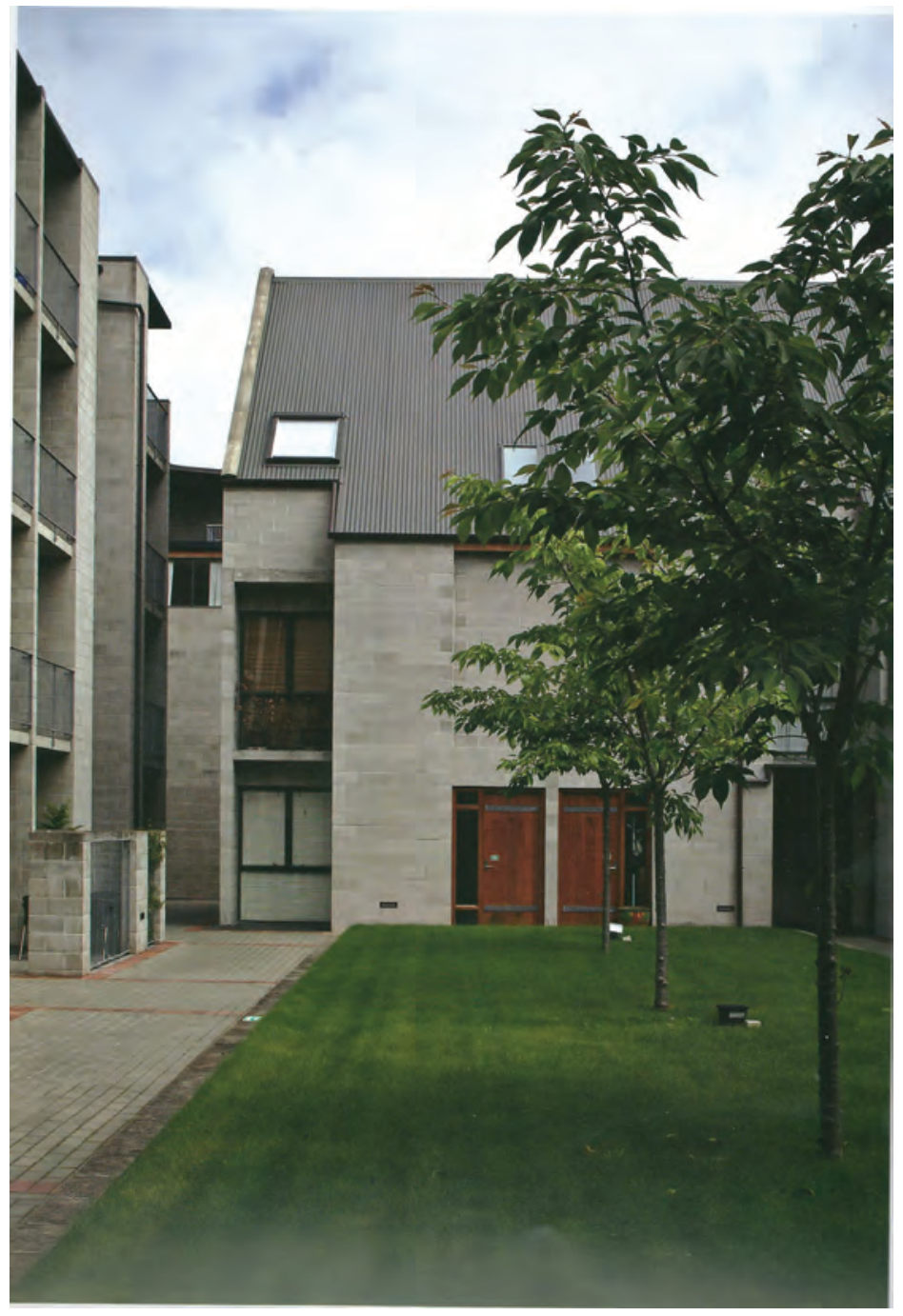




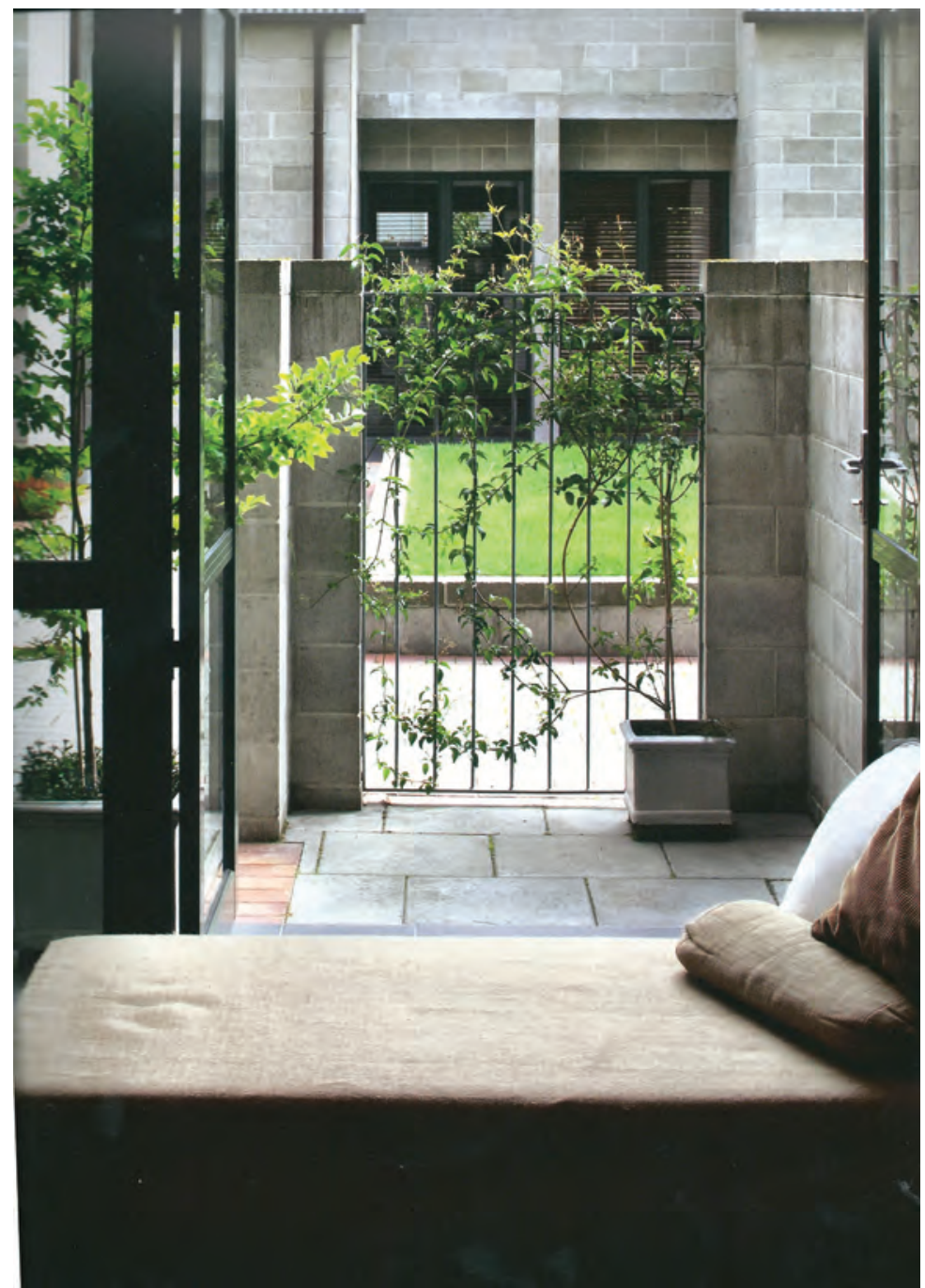

Figure D11 - St Mary's Court - Private Courtyards form a buffer to public space. Planting as screen. Photograph by Patrick Reynolds, in 'Home Work: Leading New Zealand architects' own houses' p139 (used with permission) 
5.

Scheme: Trinity Apartments

Architect: Architectus, Auckland

NZIA Award: 2007; National Award; Multiple Housing + 2008; Supreme Award.

Lessons for the Te Ara Hou Infill Development:

- Each apartment maximises natural ventilation and daylighting, and has private outdoor space.

- The 'vertical circulation node' approach avoids long corridors along edges of apartments maximising amount of exterior envelope available to apartments. This allows many of the apartments to open at two ends.

- Vertical circulation is secure (excluding public from outside main doors to apartments)

- Significant provision of private outdoor spaces, running lengths of apartment edges.

Figure D12 - Trinity Apartments - Generous strip of decking

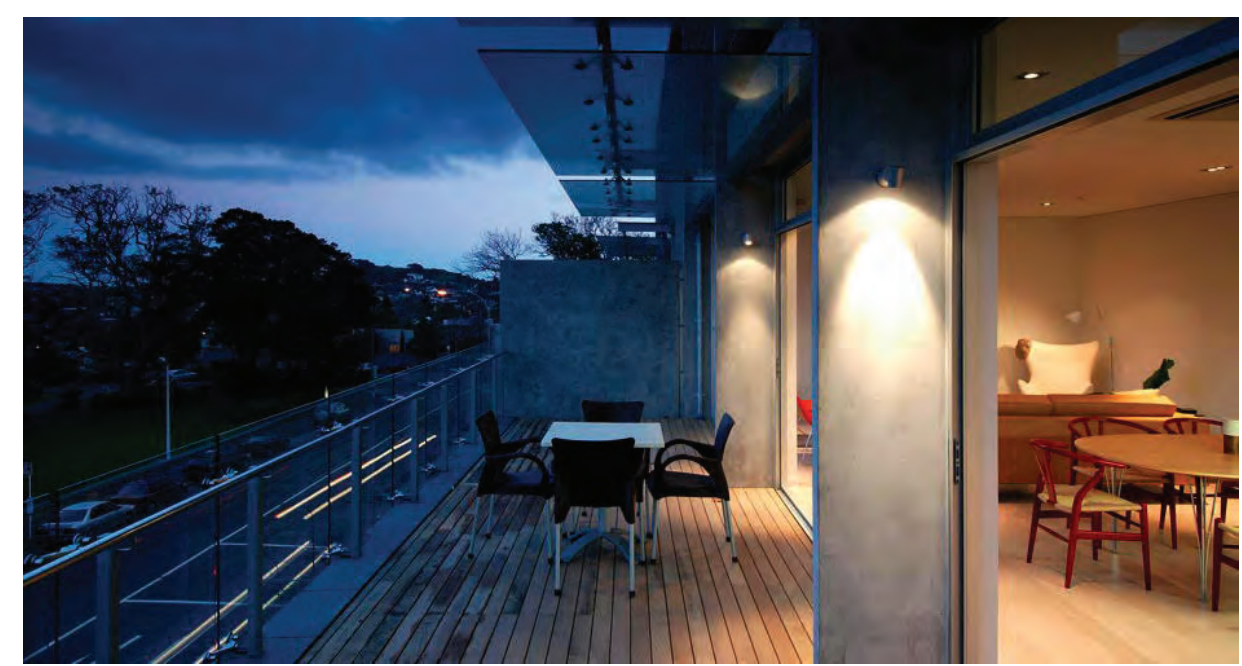




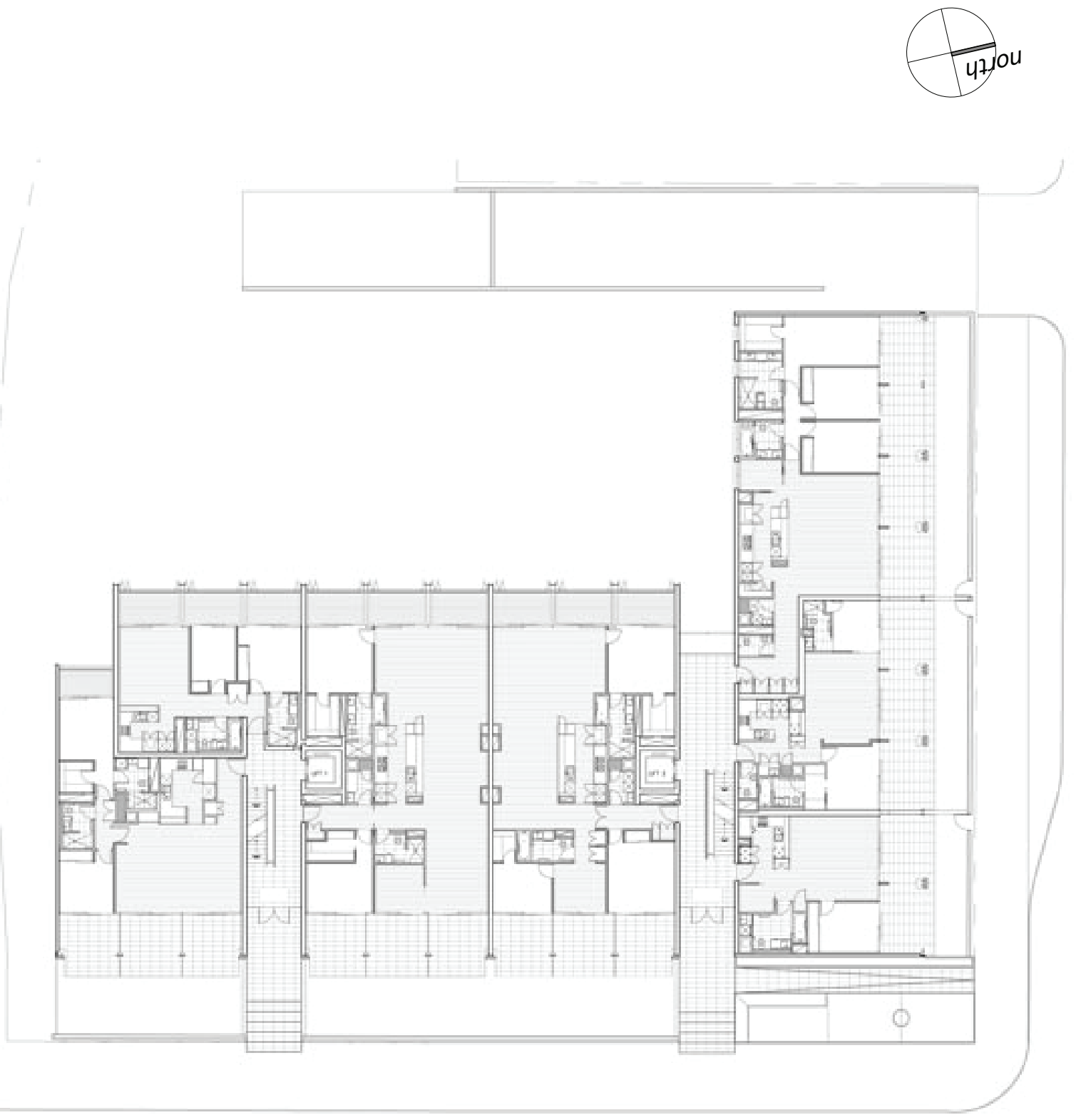

Figure D13 - Trinity Apartments - Typical Floor Plan 


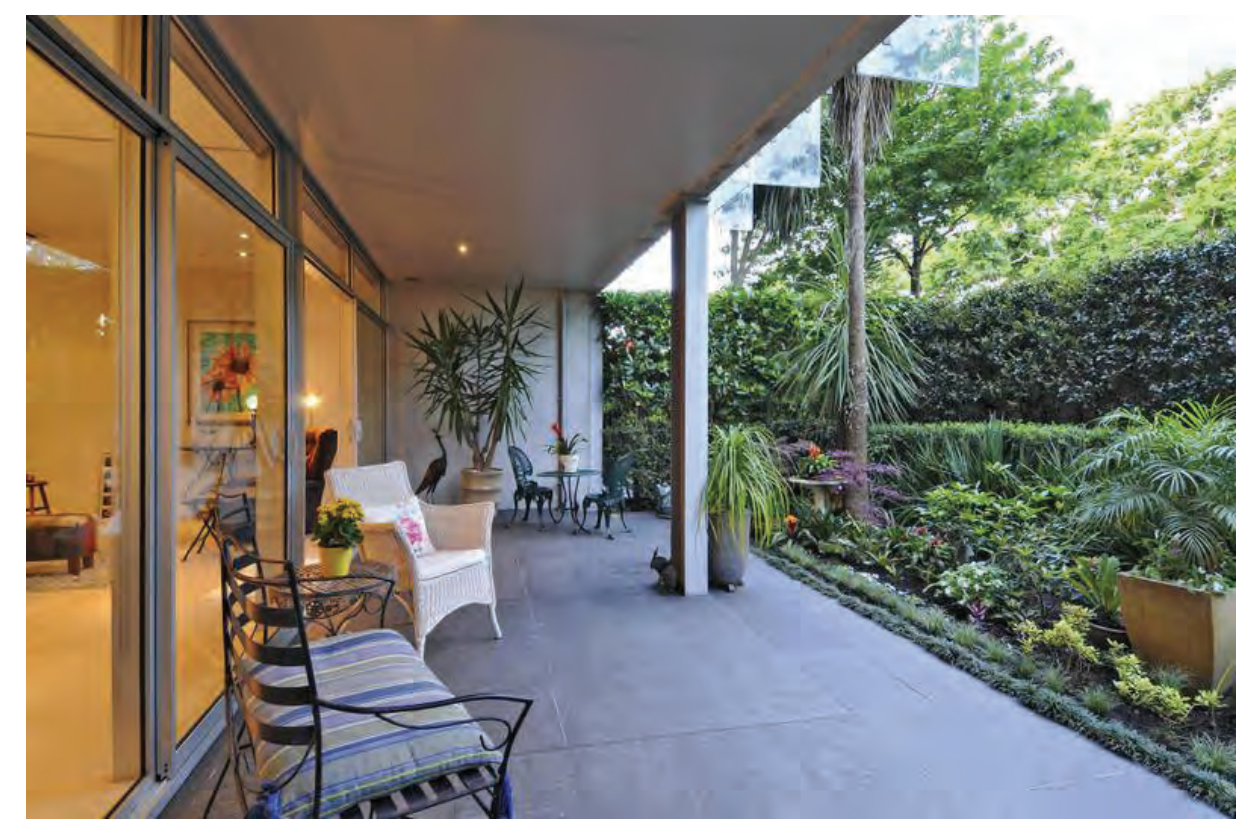

Figure D14 - Trinity Apartments - Seclusion and privacy paramount in design of ground floor outdoor spaces (photo used with permission from Real Estate Agent) 
6.

Scheme: Gore St Housing (Melbourne)

Architect: Kerstin Thompson Architects

NZIA Award: N/A

\section{Lessons for the Te Ara Hou Infill Development:}

- "In designing medium density housing, Kerstin Thompson Architects privilege the shell (the building envelope) as the critical site of intervention" (Murray 22 ), as the contribution a project makes to the civic realm is vitally important. Control over finishing of interiors can be relinquished in support of this focus on the civic contribution.

- Living level is first floor (with more privacy) and ground floor is 'flexible', to become an office/studio or perhaps a bedroom. This flexibility in planning will give the project longevity as the dynamics of the neighbourhood inevitably change.

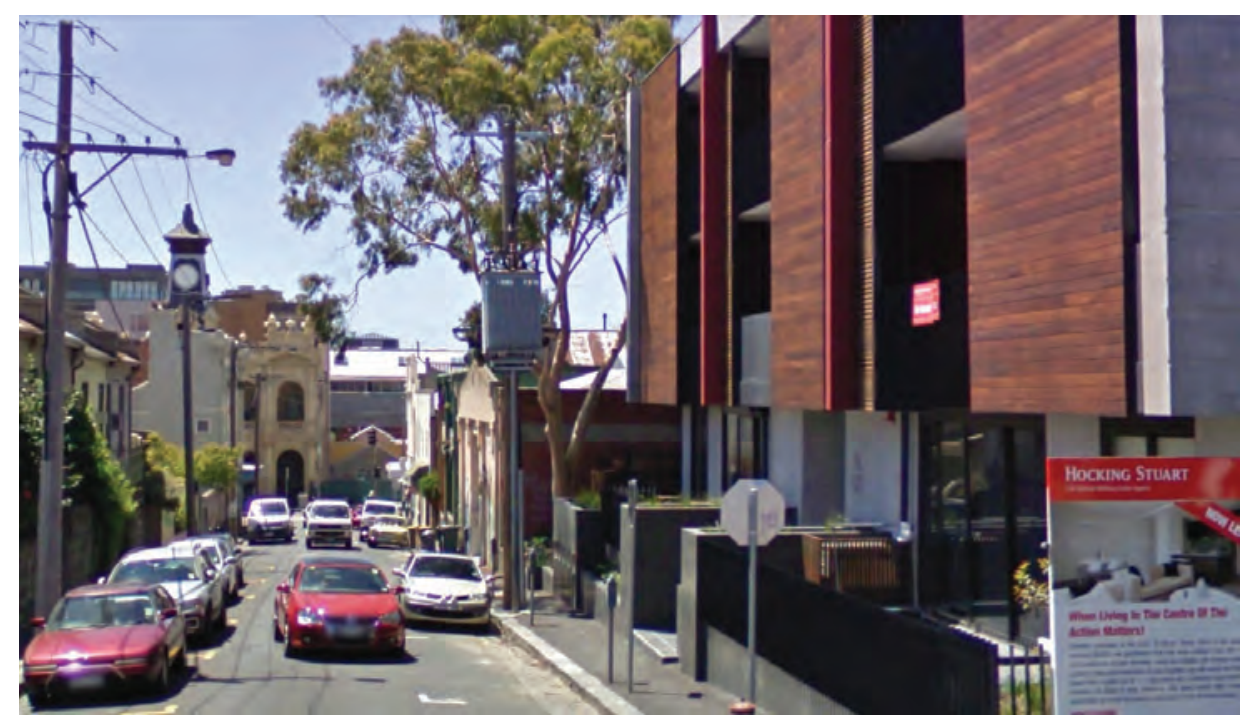

Figure D15 - Gore St Housing - Much consideration of public/private threshold at street level

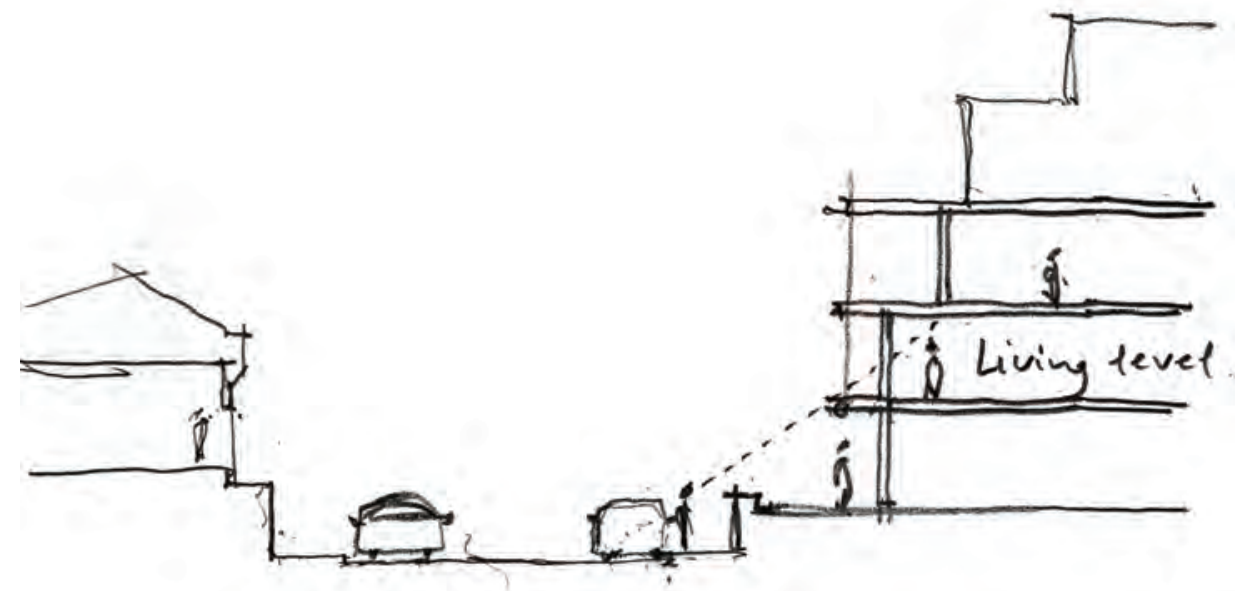

Figure D16 - Gore St Housing - Level change from footpath to semi-private outdoor space provides separation; living level more private on first floor 

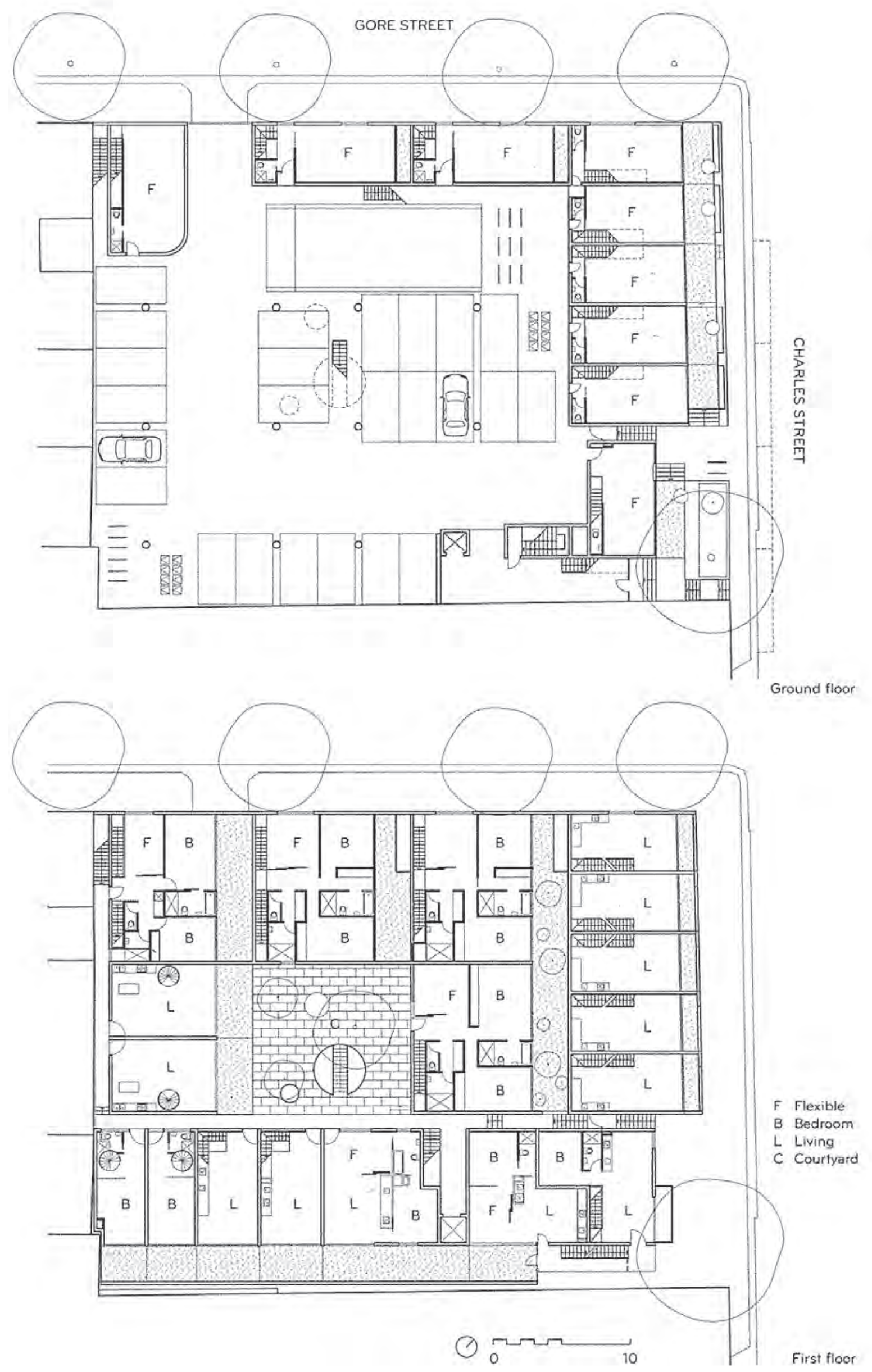


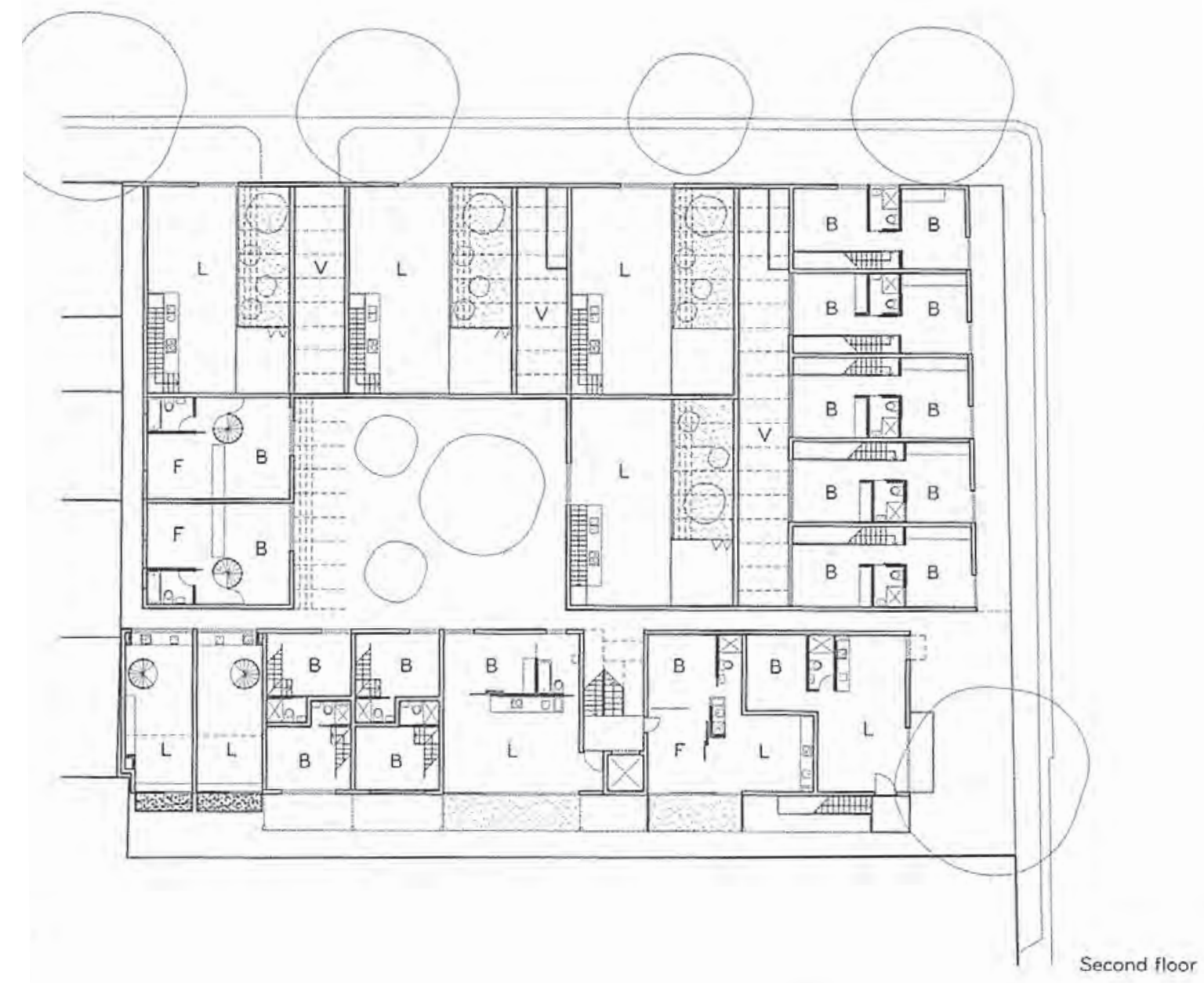

Figure D17 - Gore Street Housing Floor Plans. 
7.

Scheme: Ebor St Townhouses (Mendoza Apartments)

Architect: Unknown

NZIA Award: N/A (Reviewed on 'WellUrban' blog)

\section{Lessons for the Te Ara Hou Infill Development:}

- "... it's not dominated by cars ... Secondly the extensive planting (front gardens, street trees, window boxes and climbers) adds interest and life" 1

- "The bay windows and inter-tenancy walls help to modulate the elevation and connect it to the human scale, and the curves are derived from the retained façade of the old Ford workshop."

- Living space is on ground floor with two bedrooms on upper levels, one with balcony.

- An attractive example in terms of its street edge, although less appealing as units opens only to one side therefore capture less natural daylight and have no cross-ventilation.

$1 \mathrm{http}: / /$ wellurban.blogspot.com/2005/08/urban-eye-ebor-st-townhouses.html Accessed 23.01.11

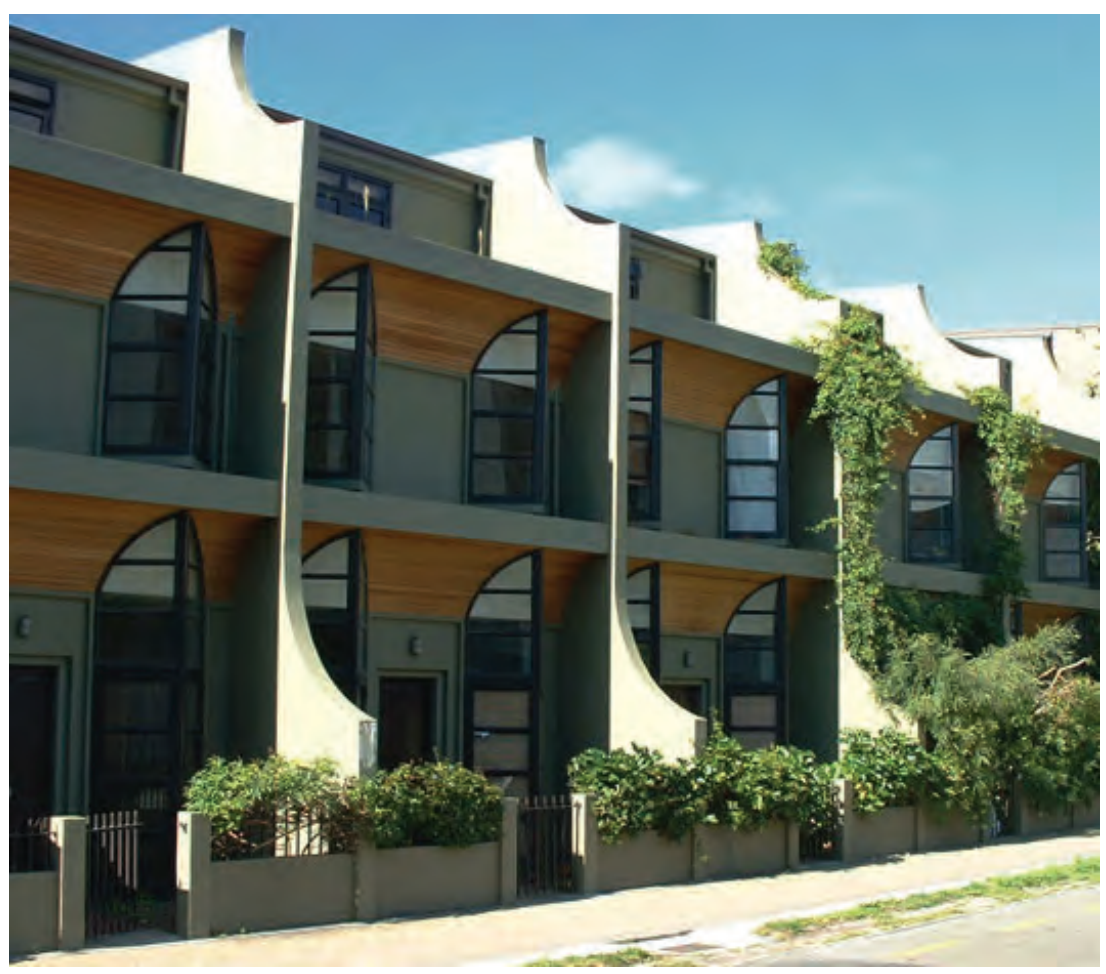

Figure D18 - Ebor St Townhouses street frontage 


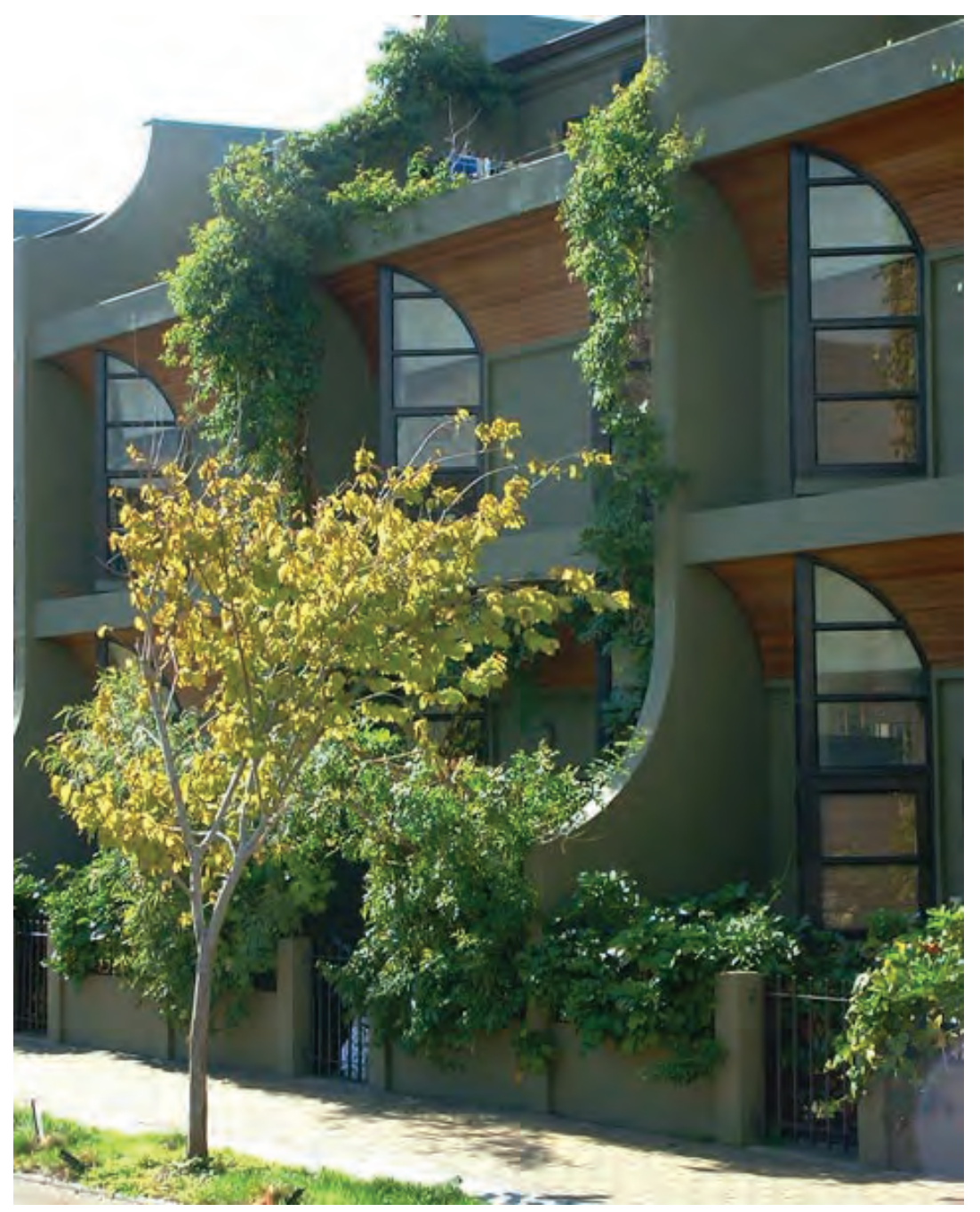

Figure D19 - Ebor St Townhouses street frontage 


\section{Appendix E - WCC community space precedents}

1.

Whare Ahuru - Glenmore St, Thorndon

A converted basement flat

- A small complex of only 16 units.

- Flat converted in 2009

- A challenge with this facility is that a part of it (the garage) is used pretty much exclusively as his art studio for one tenant. Points to a wider issue about ensuring access to spaces remains unrestricted to all tenants.

- Was originally a particularly undistinguished flat (practically a basement) which was another factor leading to its conversion into a community space.

- Activities that take place here: some tenants sew, one tenant using old garage as an art studio, and tenancy managers hold their clinics in this space on a regular basis for tenants to meet them.

Figure E1 - Whare Ahuru Locality Plan $1: 2000$
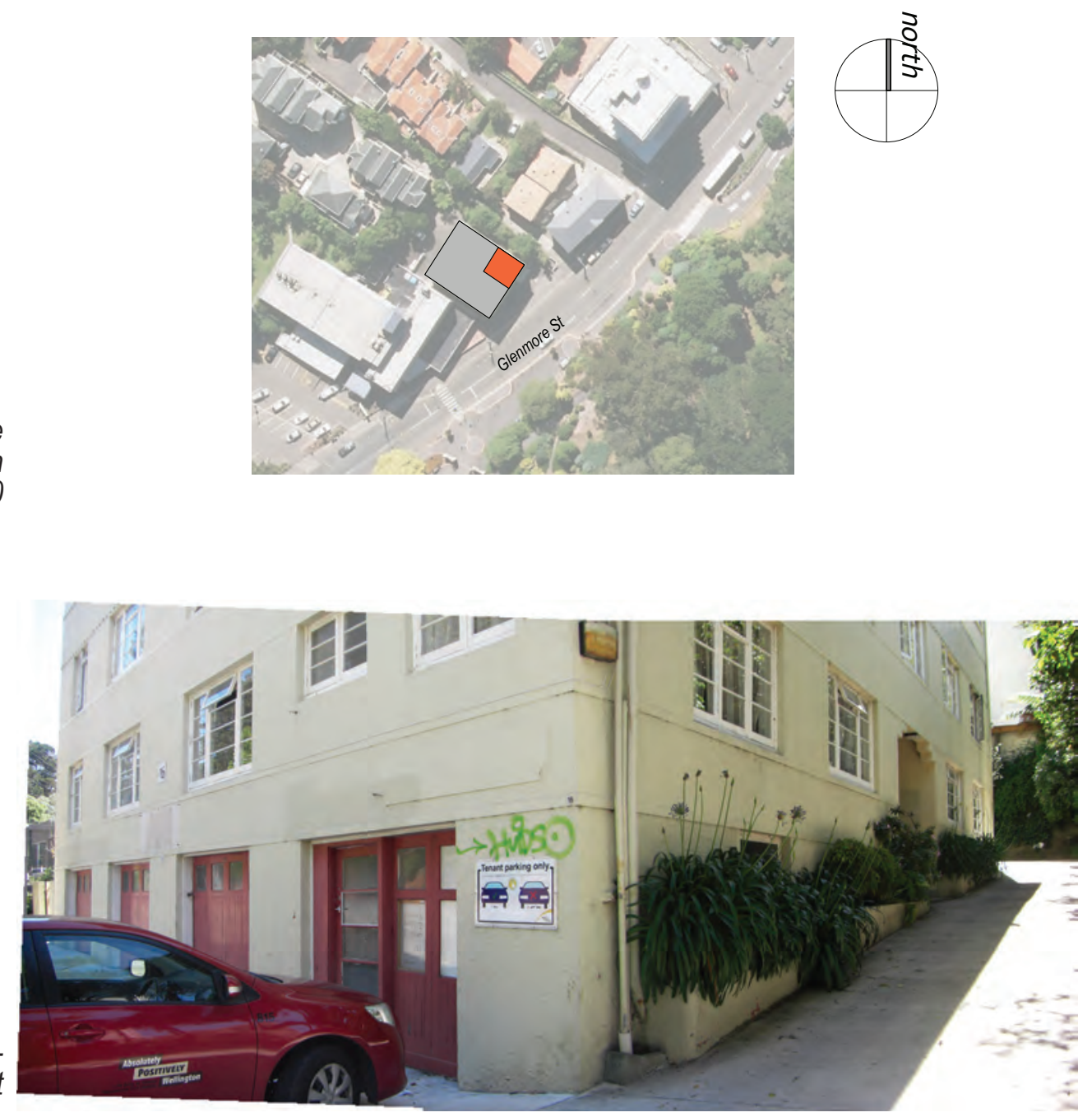


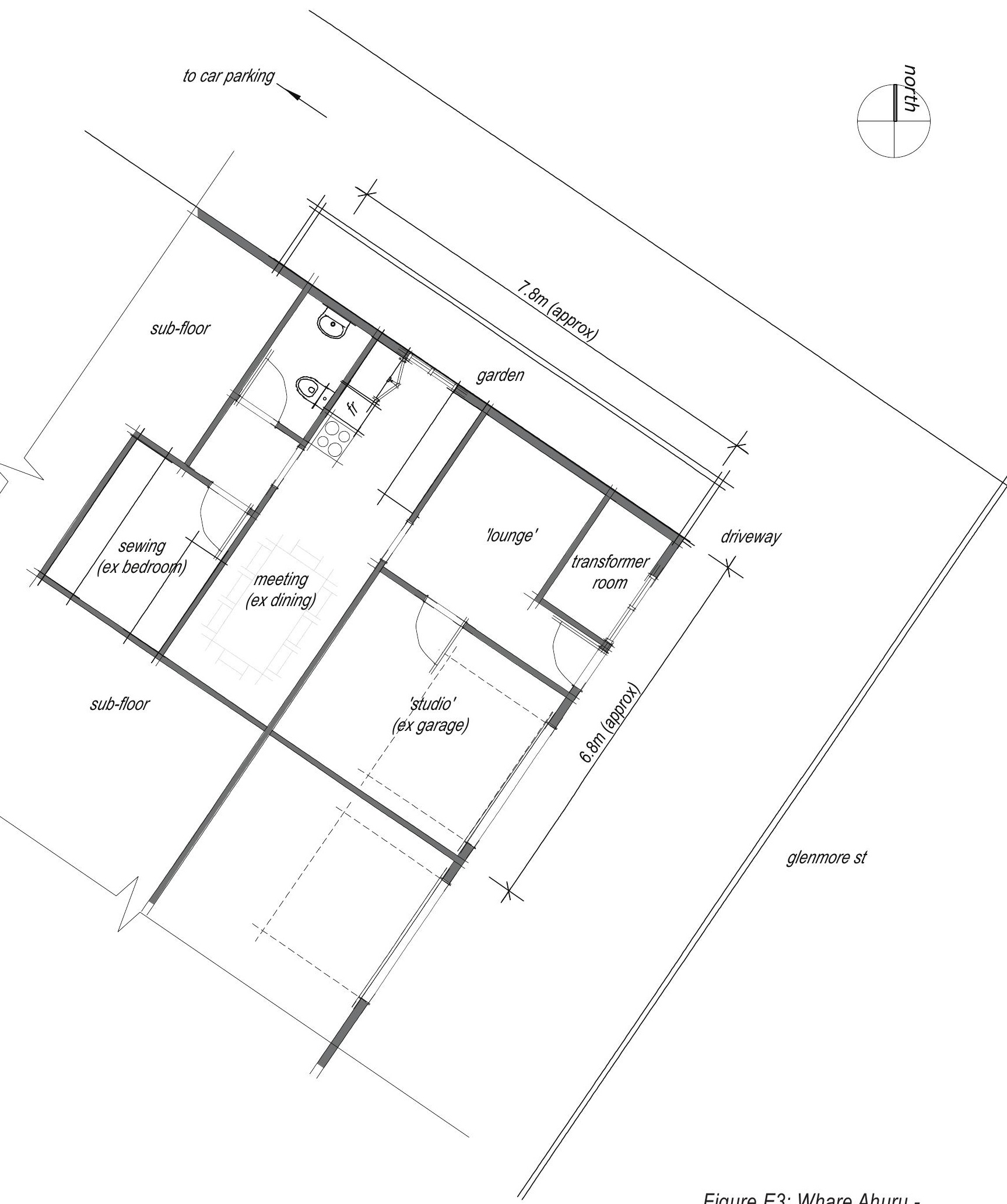

Figure E3: Whare Ahuru -

Floor Plan. Scale 1:100 
Figure E4: Whare Ahuru - looking through lounge to kitchen

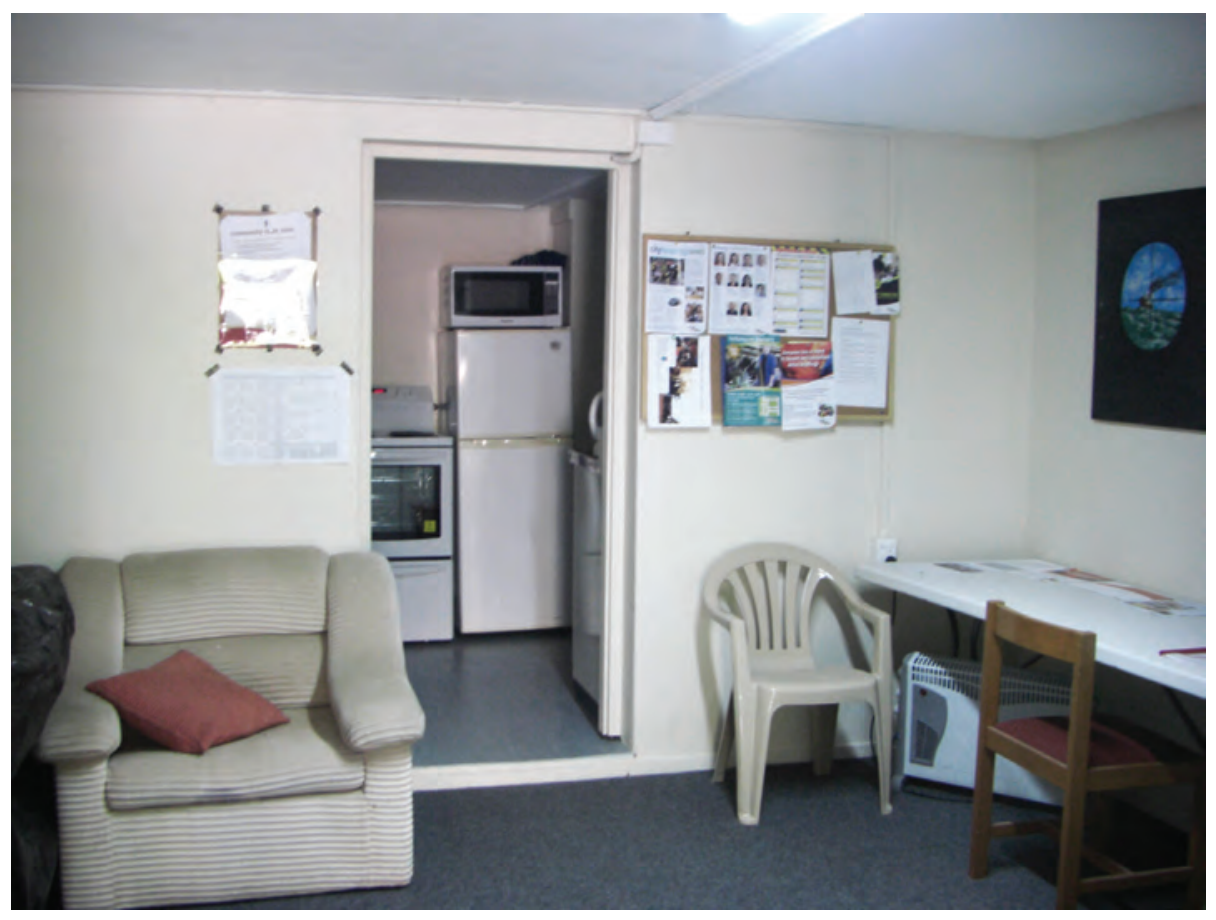

Figure E5: Whare Ahuru - sewing room 
2.

Pukehinau - Willis St, Te Aro

A converted 5th floor laundry

- Laundry converted in 2010

- Room was battened and lined (over concrete block), a false ceiling was built and a small kitchen installed.

- Cost of work was $\$ 50,000$.

- No toilet (an example of something wouldn't be possible due to regulations if this were a community room for the wider community. Being exclusively for one complex allows these facilities to sit outside of some regulations).

- Engagement with tenants here revealed that people wanted seating that could be used both indoors and outdoors (hence light plastic seats).

- This room is used for committee meetings, CAP-run workshops (e.g. 'Warm Dry Homes'), mandarin lessons, an art group etc.

- Also important to these tenants is communal use of outdoor areas (they run $4 / 5 \mathrm{BBQs}$ per year, supported by CAP). Coupled with the community room development was allocation of part of an old 'Mail and Milk' room on the ground floor to be secure storage for a BBQ.
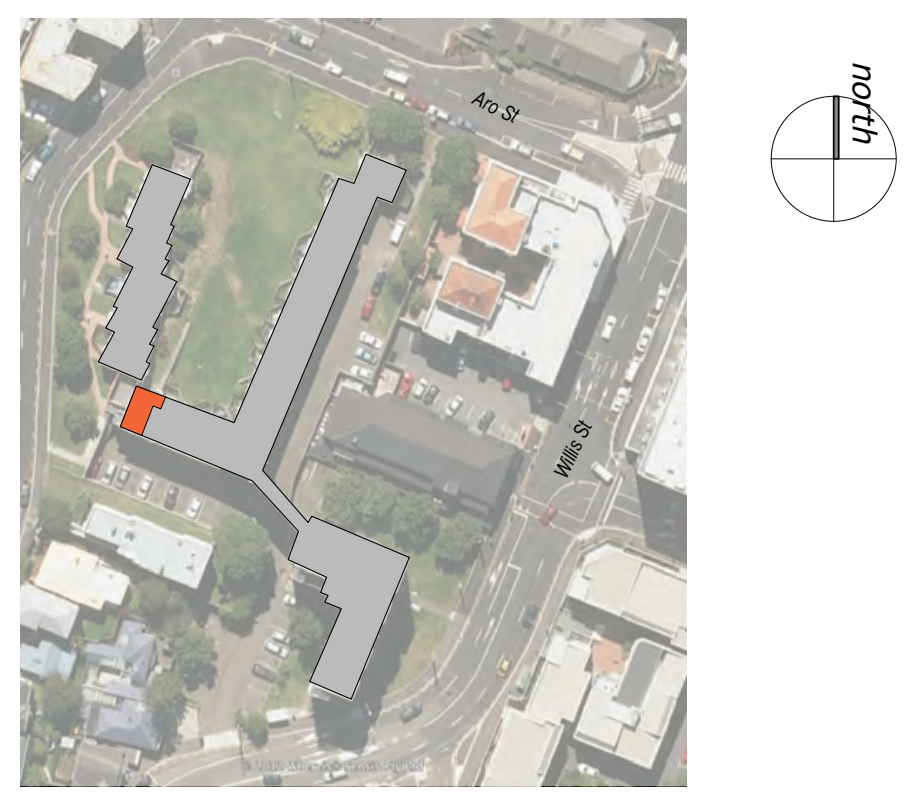


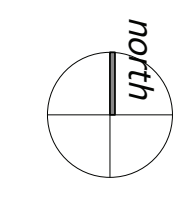

Figure E7: Pukehinau -

Floor Plan. Scale 1:100

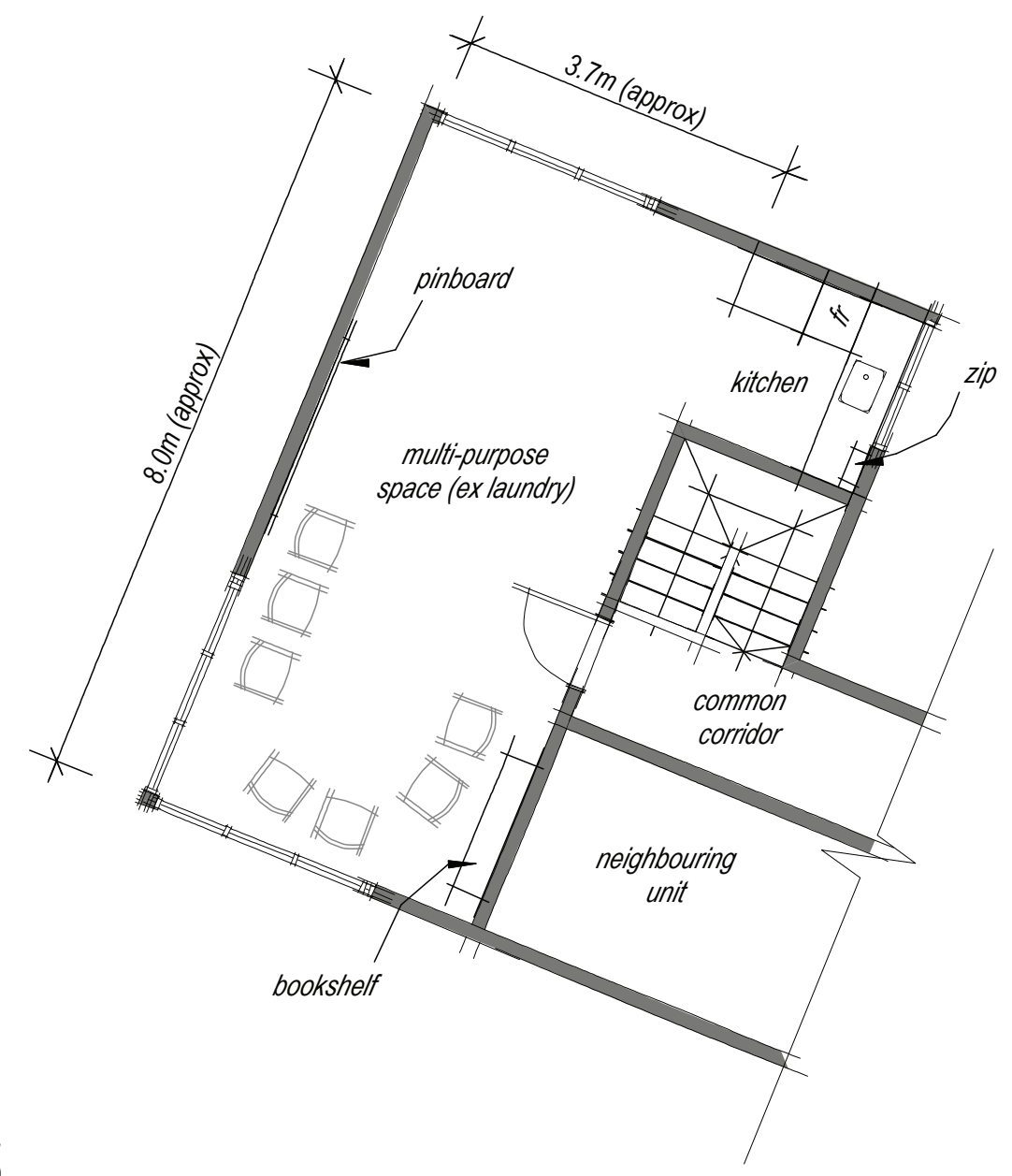




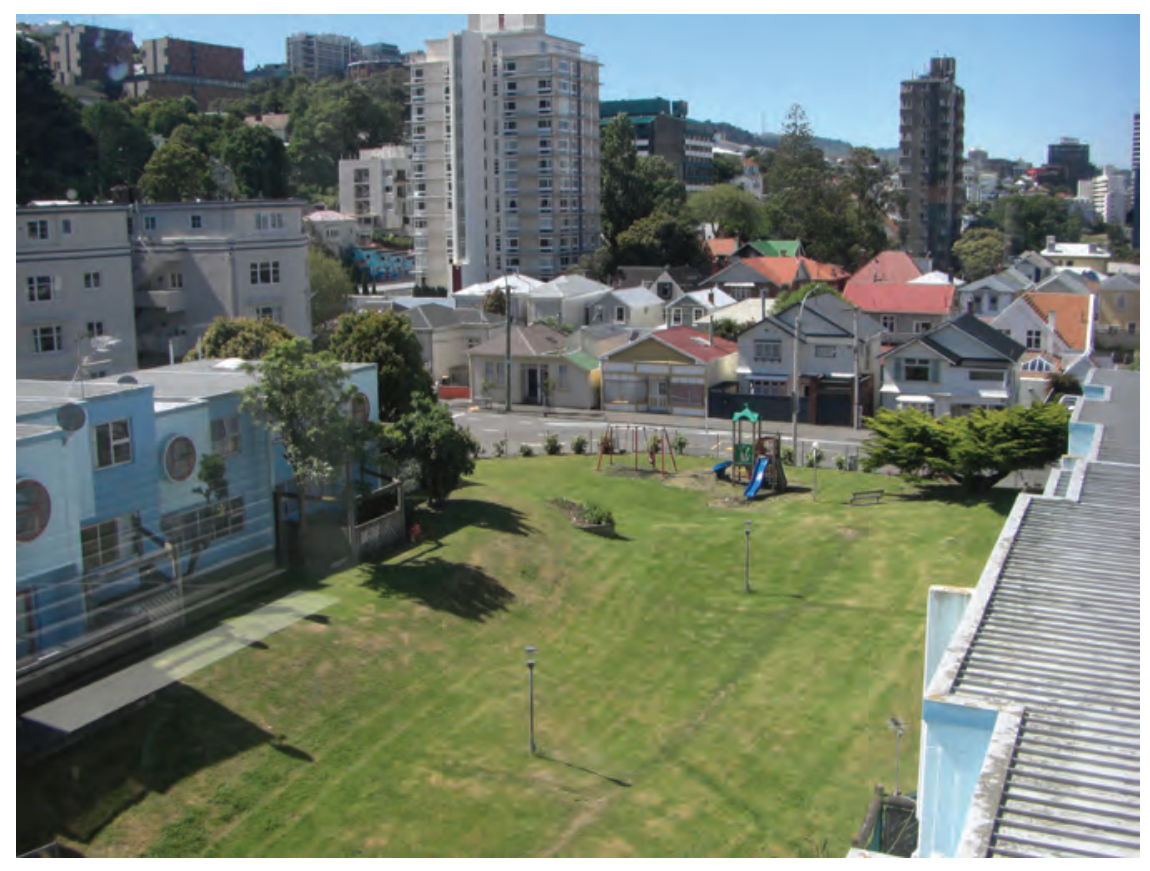

Figure E8: Pukehinau View from 5 th floor room

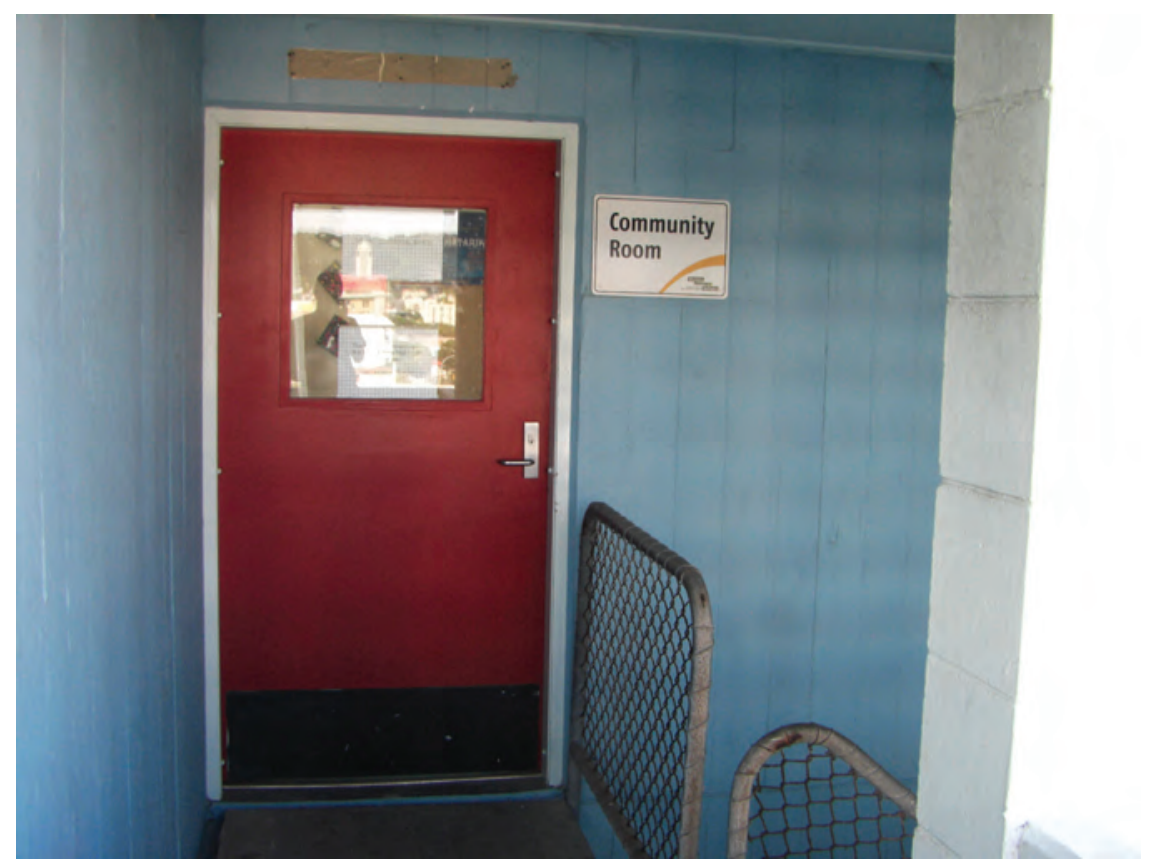

Figure E9: Pukehinau Entry door to room 
Figure E10: Pukehinau -

View towards kitchen

Figure E11: Pukehinau View looking South-west
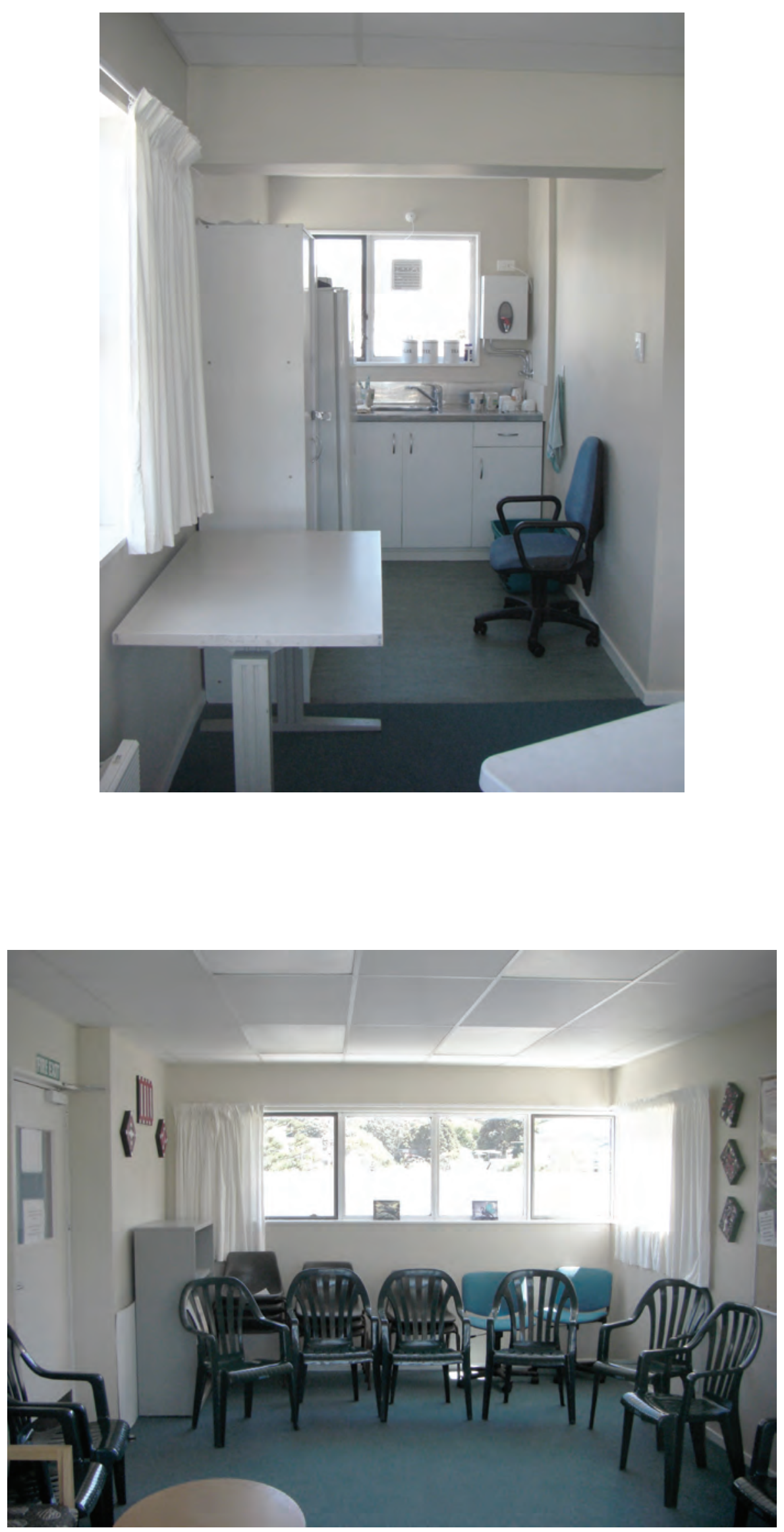
3.

Central Park - Brooklyn Rd, Mt Cook

A purpose-designed row of facilities servicing several of large complexes

- Incorporated in main upgrade works - 2011/2012 (still under construction)

- Decision was made with this facility to concentrate resources onto one large community facility that could service surrounding complexes, given their close proximity.

- These facilities service 800 units across the Central Park complex, Pukehinau, Nairn Street Flats, Berkeley Dallard and Atona.

- Tenant consultation for this facility was more substantial than with other spaces - WCC surveyed 800 units asking what tenants would like to see developed. They then held 7 focus groups with different ethnicities/age groups.

- Lots of talk in the sessions about of a gymnasium, spa pools, swimming pools.

- Eventually built:

- Gymnasium

- Computer Hub

- Toilets

- Large kitchen

- Large multi-purpose space with capacity for 100 people

- 'Hobby room' (for arts and crafts etc)

- Communal laundry and clothes drying area

- Separate tenancy manager office space and 'outside agency' space.

- Being such a large facility, the operational plan is complex (for instance, how will the gym be run/maintained?). WCC will run this facility in a more handson, centralised way than the smaller facilities.

- Gym space 'won't be Les Mills' but will have darts, table tennis, Tai Chi classes etc.

- This is third computer hub across the portfolio - others at Arlington and Newtown Park.

- Conscious move away from provision of a full workshop as management and risks were too high - instead a 'hobby room' is for 'lighter' activities like arts and crafts is being provided.

- A tenancy manager's office space (with its own kitchenette and toilet) was required to be separate for privacy reasons, and likewise for the 'outside agency' space for visiting nurses etc. 
Figure E12: Central Park - Locality Plan. Scale 1:2000
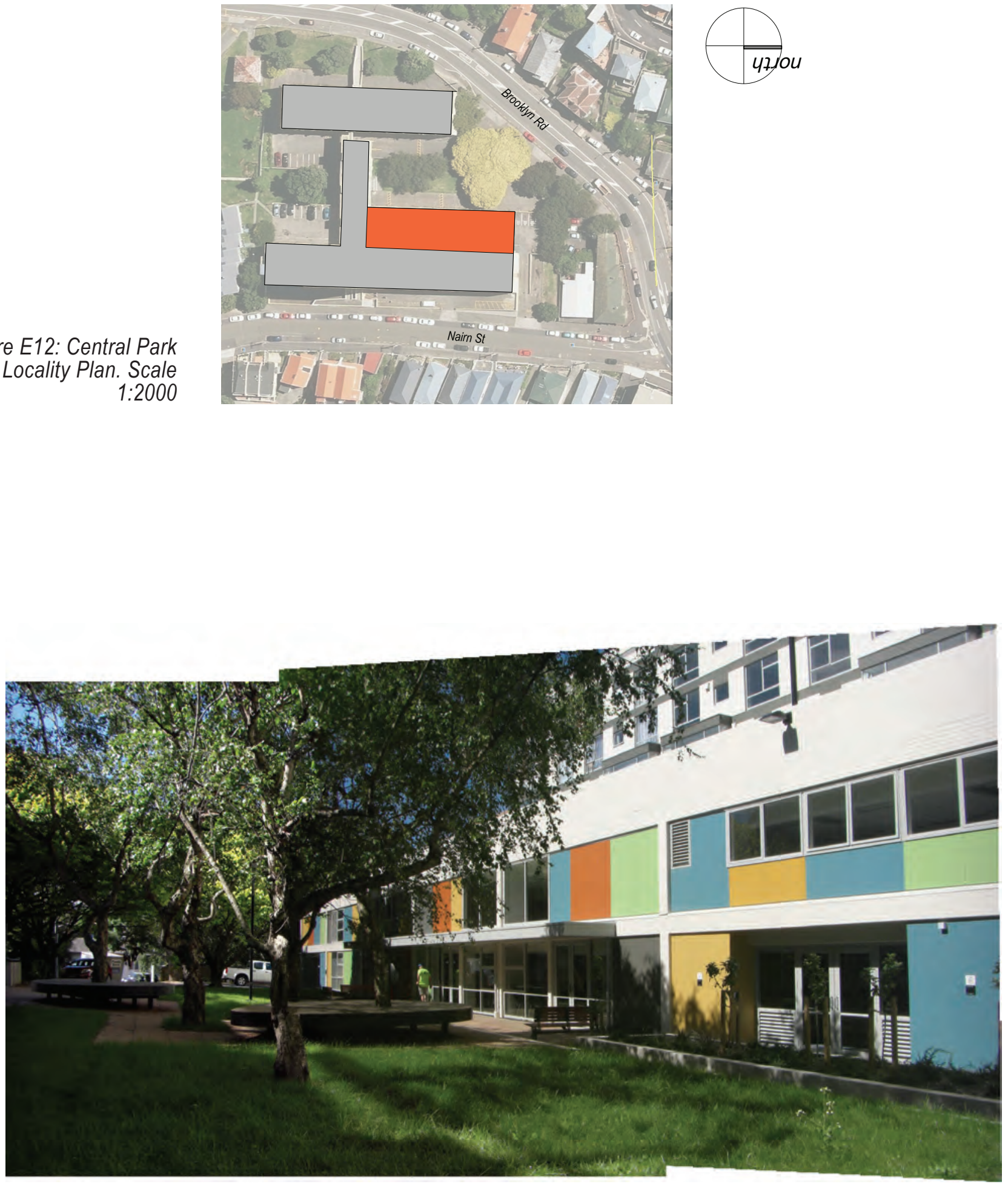

Figure E13: Central Park -

View looking North-east 


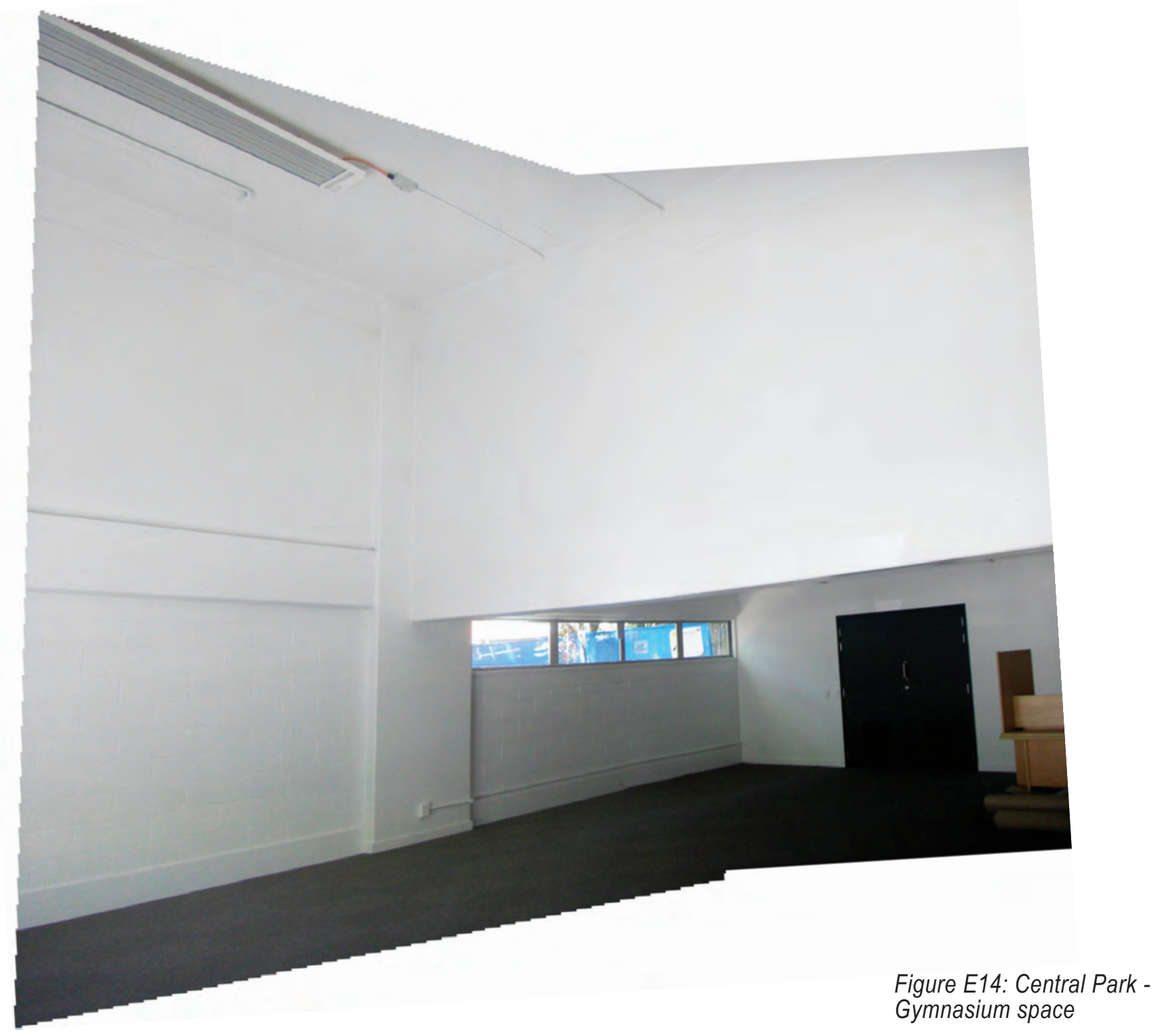




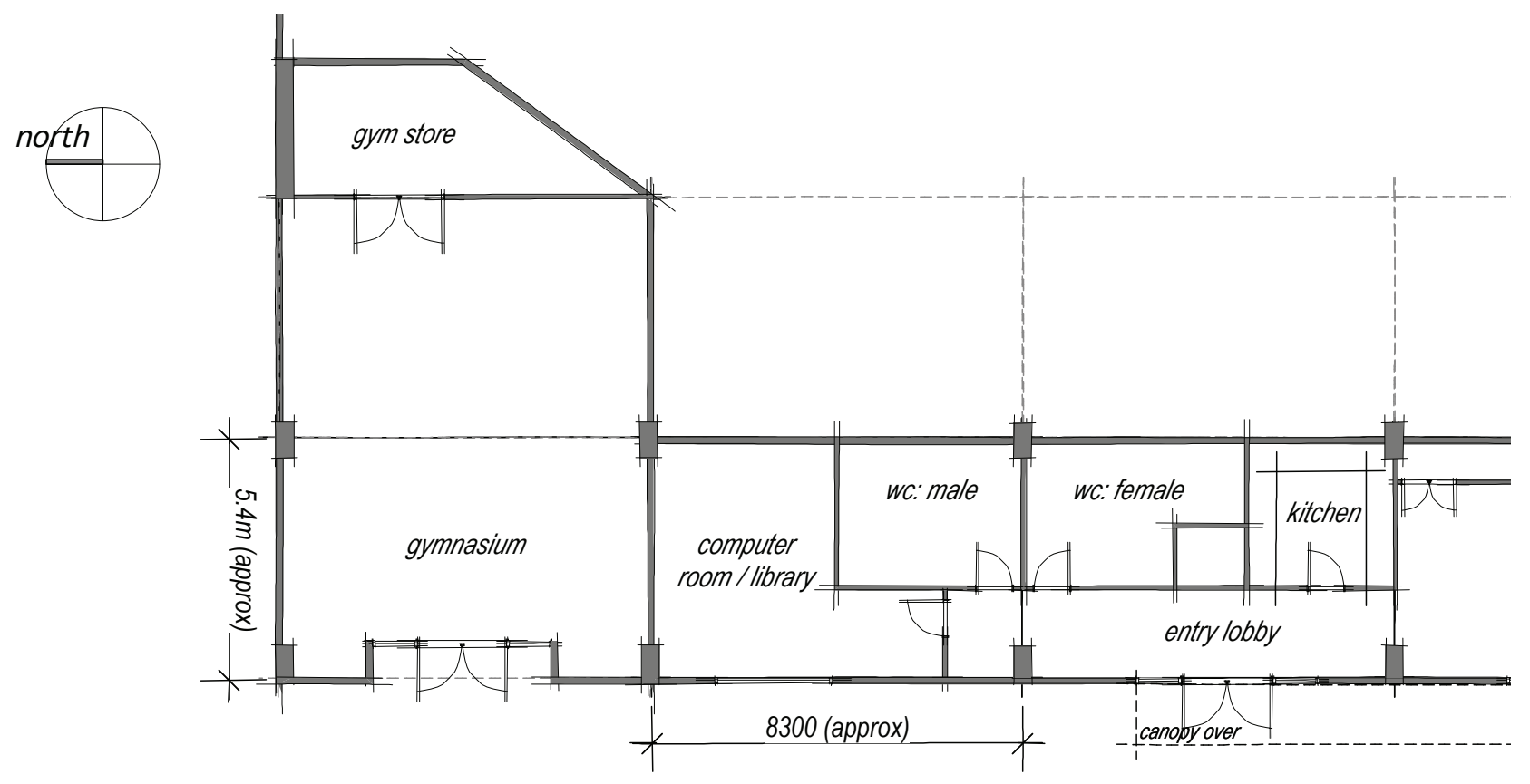

Figure E15: Central Park

- Ground floor plan. Scale

1:200

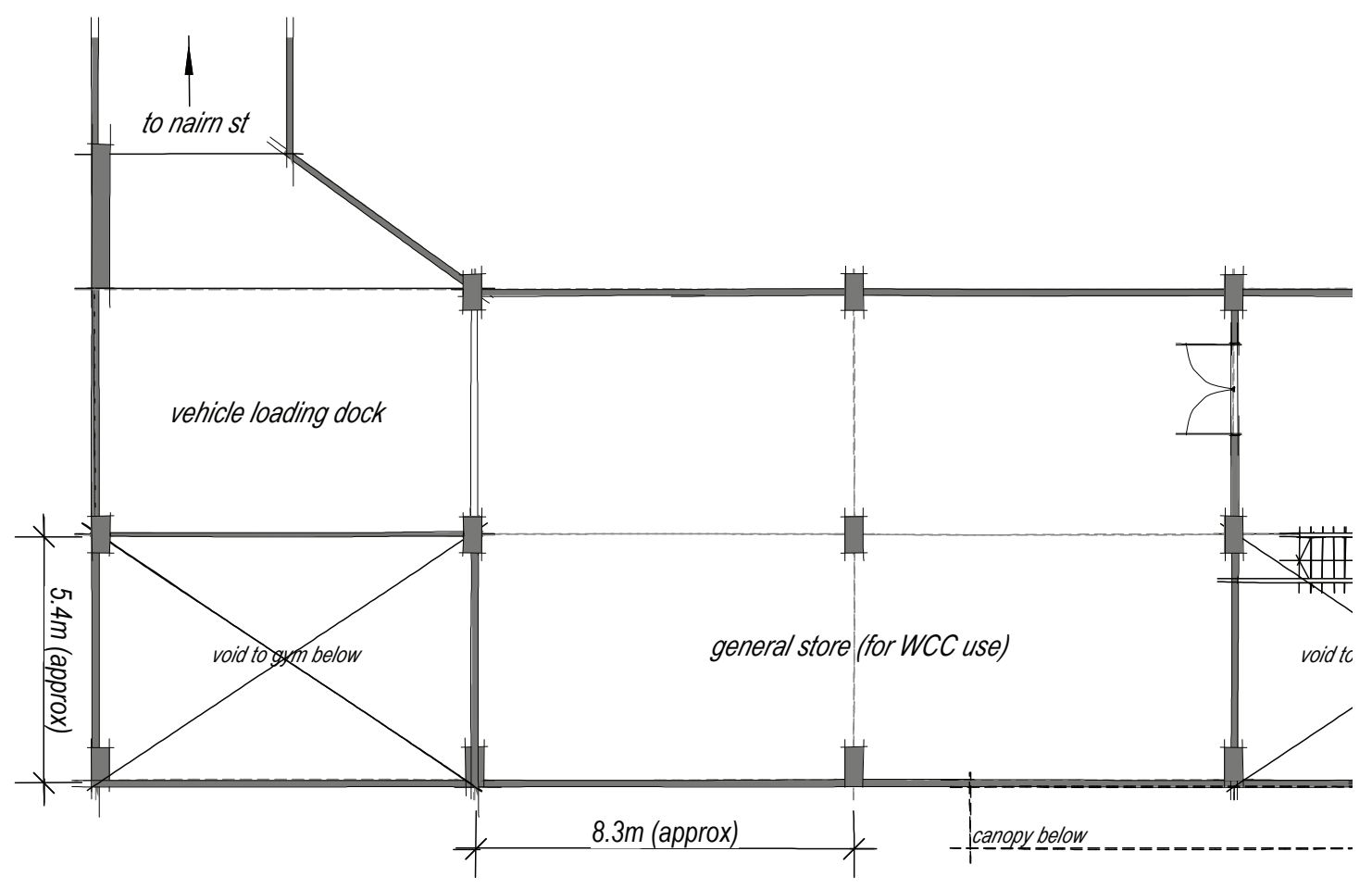

Figure E16: Central Park

- First floor plan. Scale

1:200 

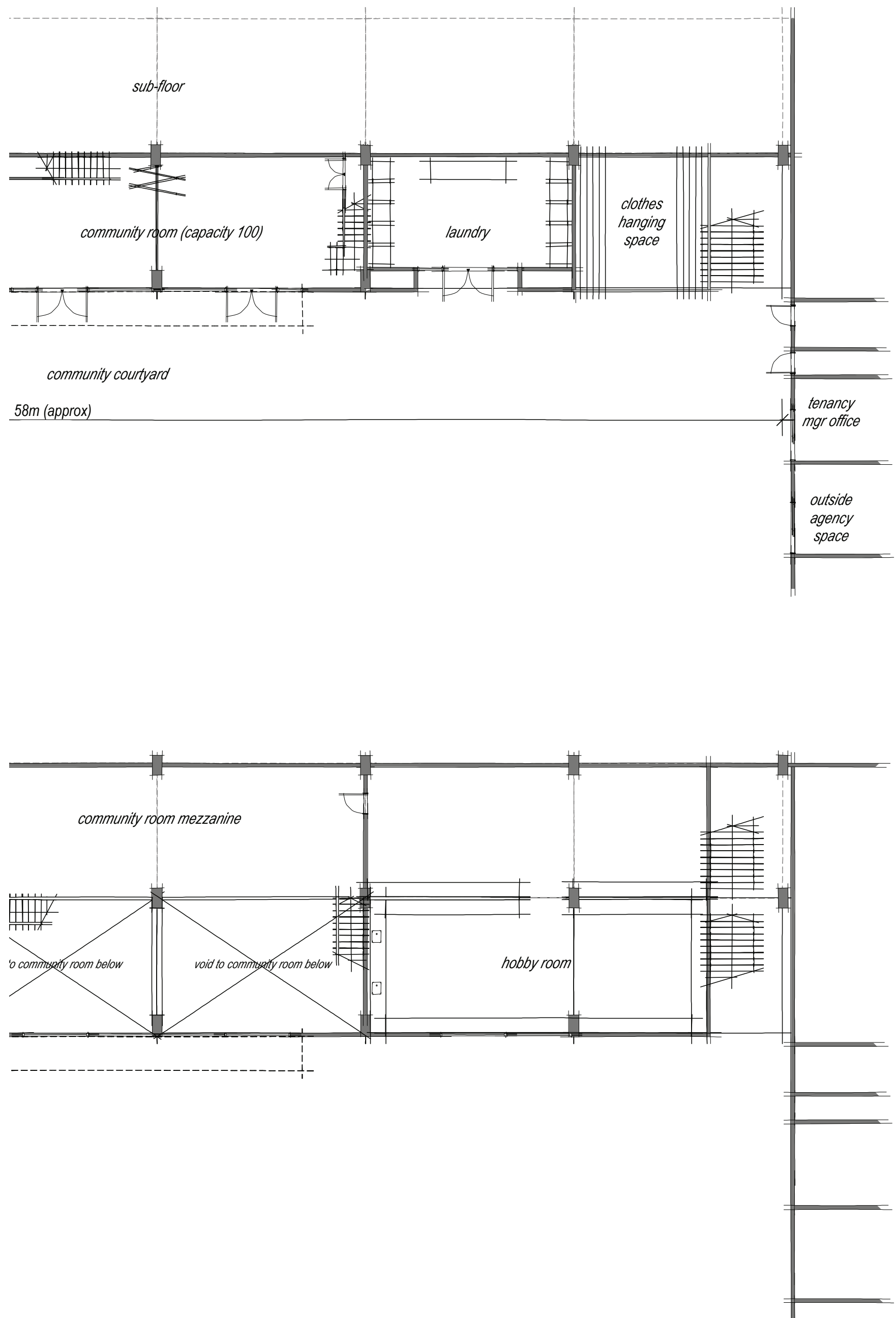


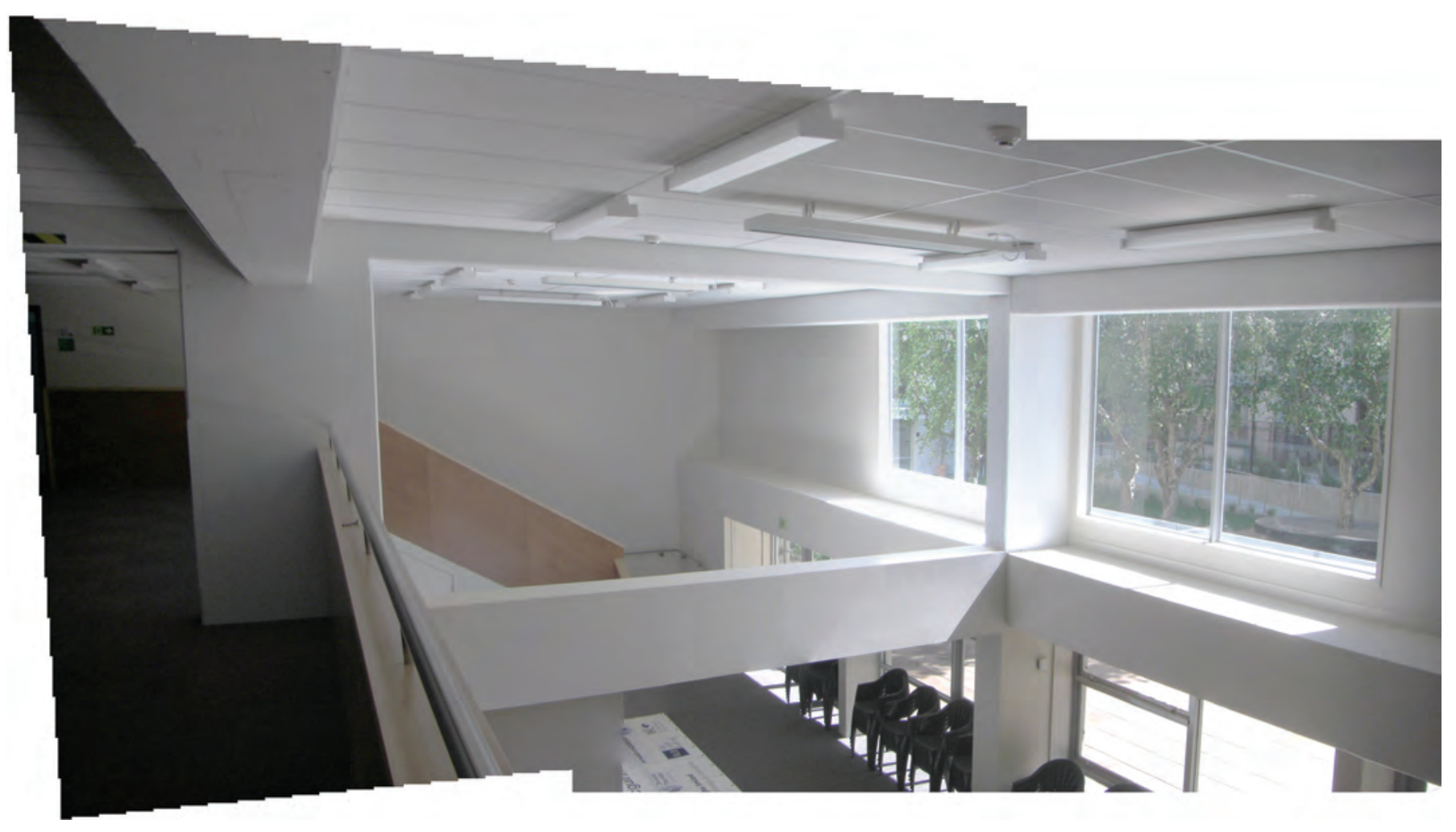

Figure E17: Central Park - View over 'community

room 


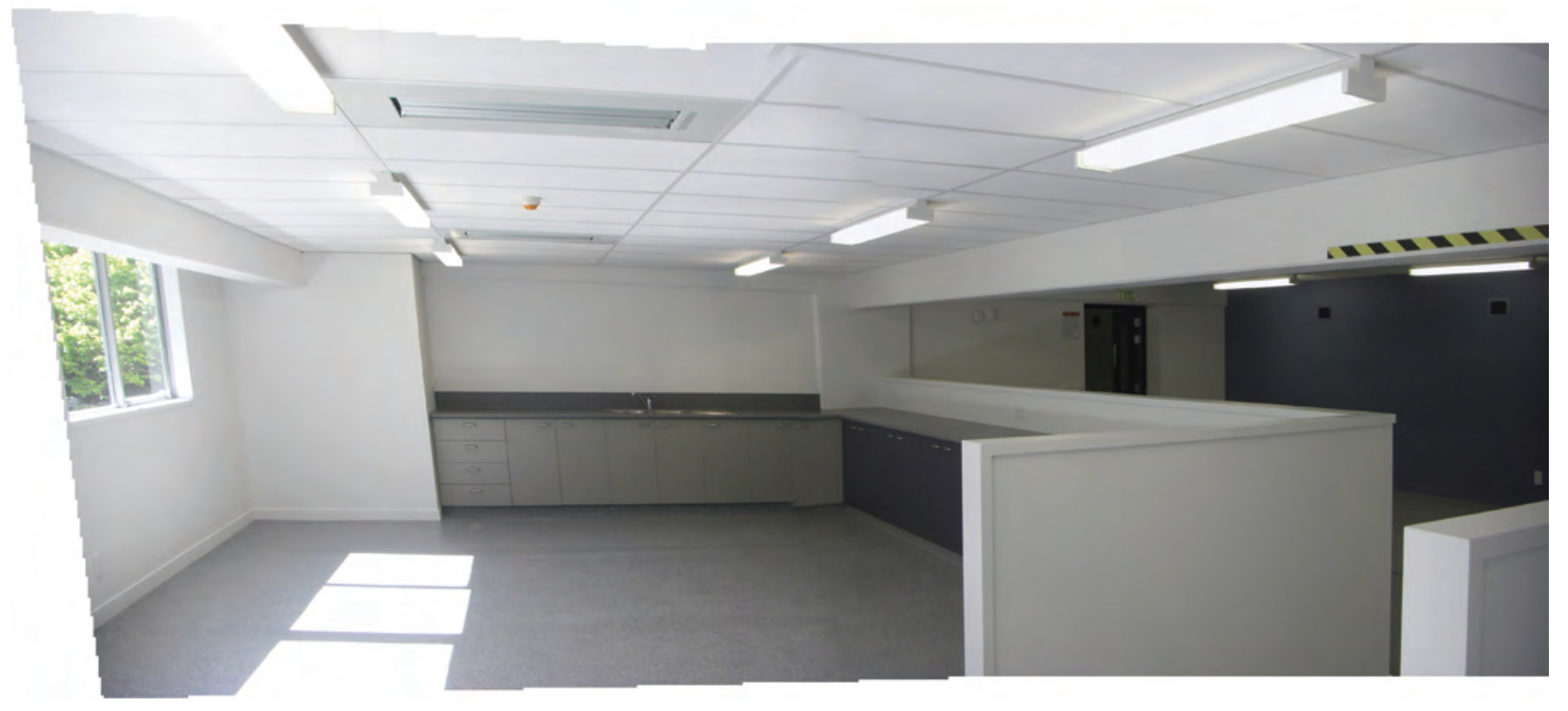

Figure E18: Central Park Hobby room 
4.

Hansen Court. John St, Mt Cook

A converted stand-alone unit relocated from another complex.

- Incorporated in main upgrade works - 2011/2012 (still under construction)

- Unit relocated from Regent Park complex which was demolished and re-built. Relocating an existing building proved to be a significantly cheaper approach.

- Includes a general community space (capacity for 50 people) with kitchen, a separate private tenancy manager's office, toilets and a communal laundry.

- Small lawn in front of the building. An area for vegetable gardening is proposed to the south of the building.

- Building placed over part of an existing car parking area. It is located centrally between the three parts of the complex - the tower (to the North), the podium (to the East) and 'three sisters' (to the South-West).

Figure E19: Hansen Court - Locality Plan. Scale 1:2000
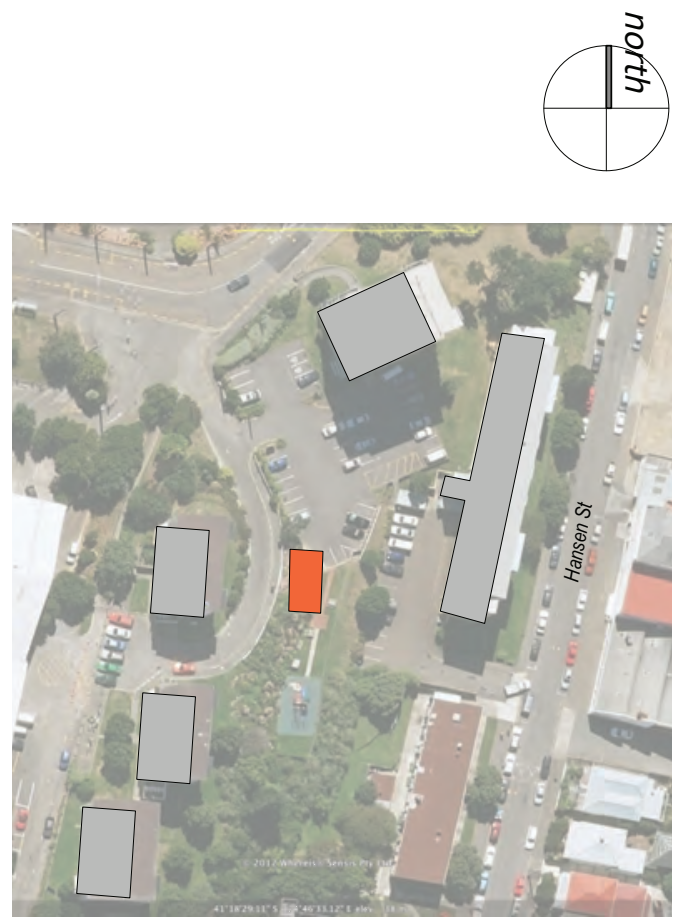


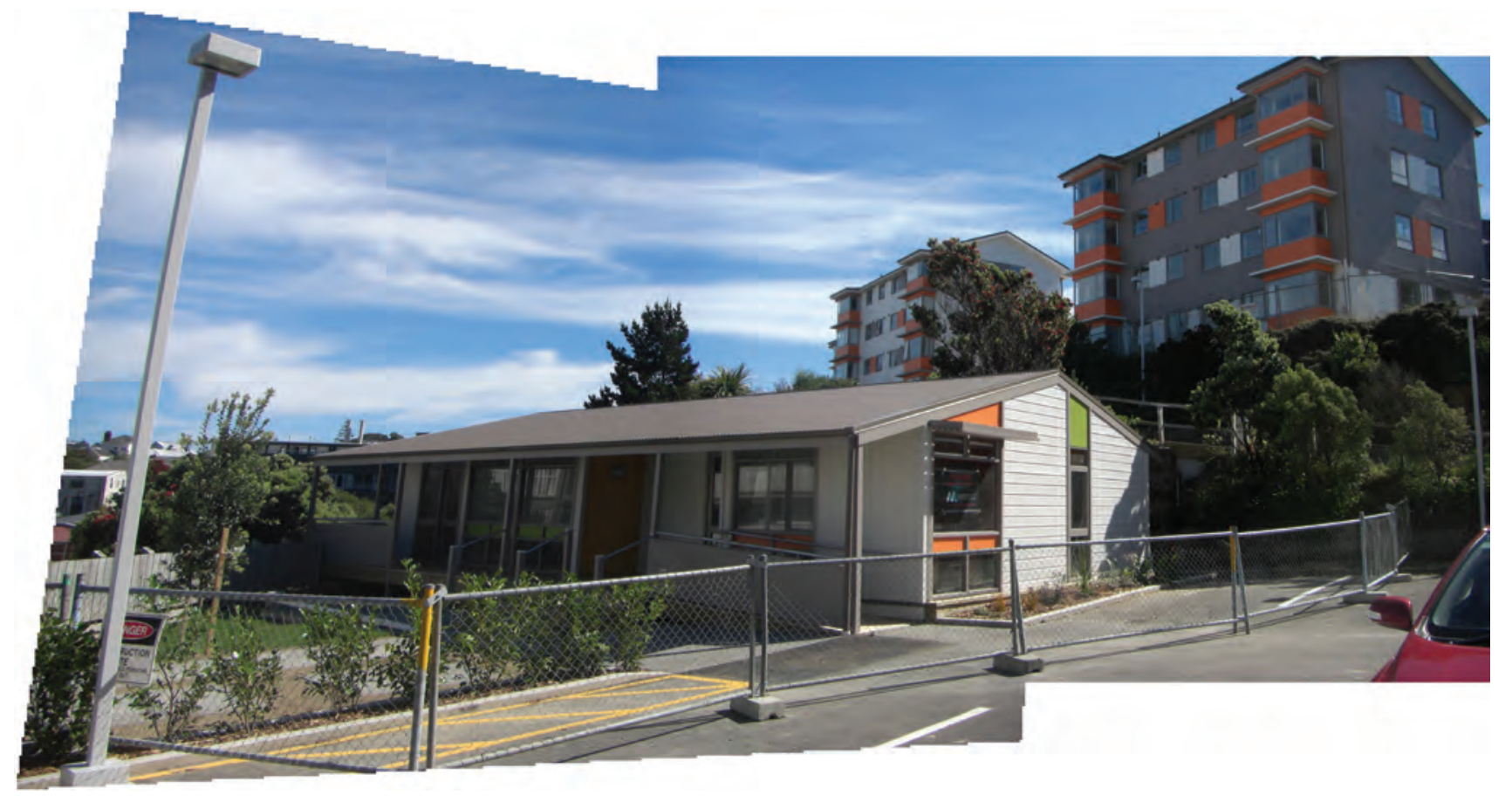

Figure E20: Hansen

Court - View of community building 


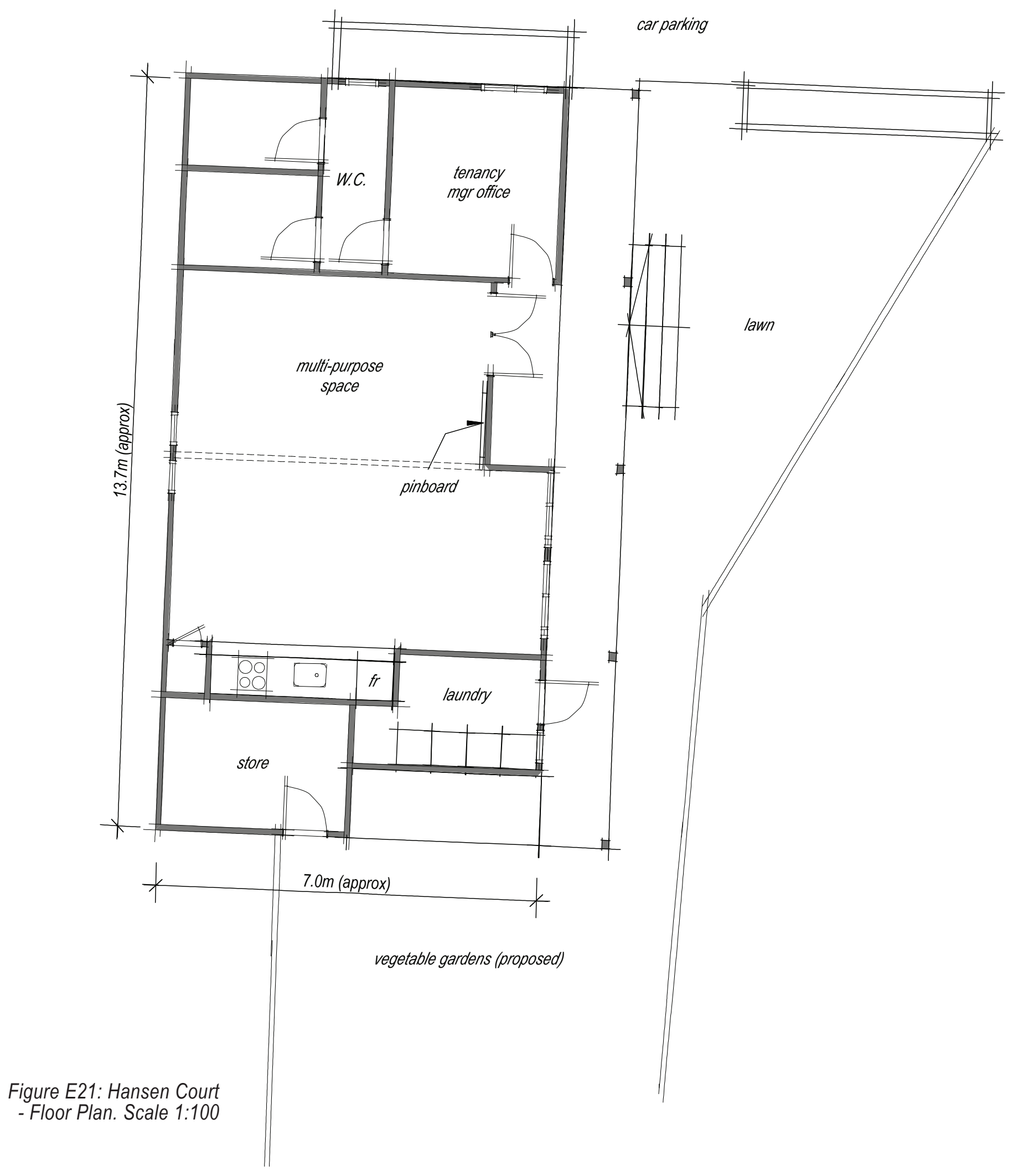

